\title{
Advanced Fuels Campaign
} FY 2011 Accomplishments Report

Fuel Cycle Research and Development 


\section{DISCLAIMER}

This information was prepared as an account of work sponsored by an agency of the U.S. Government. Neither the U.S. Government nor any agency thereof, nor any of their employees, makes any warranty, expressed or implied, or assumes any legal liability or responsibility for the accuracy, completeness, or usefulness, of any information, apparatus, product, or process disclosed, or represents that its use would not infringe privately owned rights. References herein to any specific commercial product, process, or service by trade name, trade mark, manufacturer, or otherwise, does not necessarily constitute or imply its endorsement, recommendation, or favoring by the U.S. Government or any agency thereof. The views and opinions of authors expressed herein do not necessarily state or reflect those of the U.S. Government or any agency thereof.

Cover Graphic: SEM image of as-received depleted uranium oxide from AREVA. 
INL/EXT-11-24049

FCRD-FUEL-2012-000025

Revision 0

Fuel Cycle Research and Development

\section{Advanced Fuels Campaign \\ FY 2011 Accomplishments Report}

November 2011

Idaho National Laboratory

Idaho Falls, Idaho 83415

http://www.inl.gov

Prepared for the

U.S. Department of Energy

Office of Nuclear Energy

Under DOE Idaho Operations Office

Contract DE-AC07-05ID14517 
FCRD-FUEL-2012-000025

November 2011 


\title{
Fuel Cycle Research and Development
}

\section{Advanced Fuels Campaign FY 2011 Accomplishments Report}

\author{
INL/EXT-11-24049 \\ FCRD-FUEL-2012-000025 \\ Revision 0
}

November 2011

Compiled and edited by:

Lori Braase (lori.braase@inl.gov)

Douglas Hamelin (douglas.hamelin@inl.gov)

INL Systems Engineering

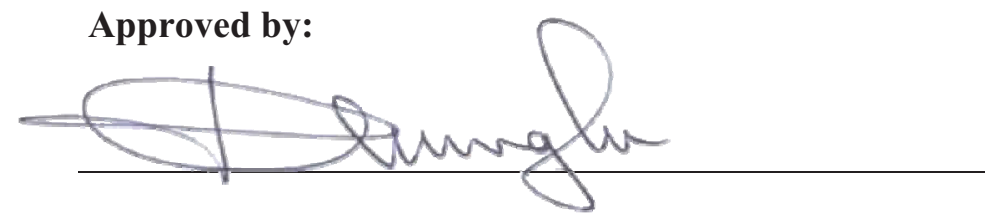

November 29, 2011

Kemal Pasamehmetoglu

Date

FCRD AFC National Technical Director 


\section{EXECUTIUE SUMMARY}

The Advanced Fuels Campaign (AFC) under the Fuel Cycle Research and Development (FCRD) program is responsible for developing fuels technologies to support the various fuel cycle options defined in the DOE Nuclear Energy Research and Development Roadmap, Report to Congress, April 2010. The fiscal year 2011 (FY 2011) accomplishments are highlighted below.

The AFC uses a "goal-oriented science-based approach" aimed at a fundamental understanding of the fuel fabrication methods and fuel performance in the reactor, enabling the pursuit of multiple fuel forms for the future fuel cycle options. This approach comprises fundamental experiments, theory and advanced modeling and simulation. The modeling and simulation activities for fuel performance are carried out under the Nuclear Energy Advanced Modeling and Simulation (NEAMS) program and the AFC activities are closely coordinated with the NEAMS team.

AFC management and integration activities included continued support for International collaborations, primarily with France, Japan, European Union, Russian Federation, Republic of Korea, and China. A Cooperative Research and Development Agreement (CRADA) was signed with the Republic of Korea to jointly develop metallic fast reactor fuels and the corresponding recycling technologies. Quarterly campaign meetings focused on reviewing technical progress and overall program integration. Three quarterly meetings were held jointly with other FCRD campaigns. They include Separations and Waste Forms, Systems Analysis, and Modeling and Simulation. Industry involvement, via Department of Energy (DOE) Task Orders, increased in both the quarterly meetings and the Advanced LWR industry meetings sponsored by AFC. As a result of the Fukushima event, an increased emphasis was placed on the development of advanced LWR fuels with enhanced accident tolerance.

Industry meetings specific to the development of advanced LWR fuels were held in conjunction with the AFC quarterly meetings. The Advanced LWR Fuel Advisory Committee was chartered to provide input to the National Technical Director (NTD) on industry-valued objectives for fuel development. Representatives included utilities, vendors, and the Electric Power Research Institute (EPRI). They issued a request for oral proposals and developed criteria to make recommendations for the development of advanced fuels.

The technical accomplishments are reported under the following research and development (R\&D) areas:

- Analytic Support, C. Unal, LANL, Technical Lead

- Metallic-Based Fuels Technologies, J. R. Kennedy, INL, Technical Lead

- Ceramic-Based Fuels Technologies, K. McClellan, LANL, Technical Lead

- Coated Particle Fuels Technologies, L. L. Snead, ORNL, Technical Lead

- Core Materials Technologies, S. Maloy, LANL, Technical Lead

- Irradiation Testing Technologies, S. L. Hayes, INL, Technical Lead

\section{Analytic Support}

Analytic Support is a new technical area for AFC focused on the analysis of the impact of novel fuel forms on reactor operations and the development of fuel fabrication models, such as sintering, casting, etc. In addition, this is the primary interface with NEAMS to identify fuels and materials data needs. Some of the specific accomplishments include:

The requirements of the metal and oxide fuel performance analysis tool were developed. The BISON metallic rodlet demonstration model was developed with accurate geometrical dimensions of the 
components and the as-run linear heat generation rate to provide representative centerline temperature results for the FCRD irradiation experiments.

A database for 10 Metallic Fuel Pins from experiment X425 irradiated at EBR-II, containing metallic fuel elements, was generated as validation data for the BISON-METAL analysis tool. The database contains detailed operating conditions for 10 pins from this experiment at different stages of irradiation and corresponding experimental measurements of cladding strain.

Calculations were performed to evaluate the performance and safety characteristics of a proposed concept by Logos Nuclear Systems based on the utilization of tristructural isotropic (TRISO) particle fuel in selected locations in a pressurized-water reactor (PWR) assembly to burn the transuranics (TRU) from light-water reactor (LWR) used nuclear fuel (UNF). The TRISO particles would be in silicon carbide or graphite compact/stick that would be located in conventional Zircaloy clad.

FLOW3D simulations of the casting process of metallic fuels in the bench-scale caster (BSC) filled with compressible Argon gas was developed using FLOW3D's adiabatic bubble model. We evaluated the ability of FLOW3D to run reasonably resolved simulations of the BSC and assess the magnitude of the effects of surface tension and gas compressibility. The scalability of FLOW3D to large highly-resolved simulations was also investigated. The performance of coarse and fine FLOW3D numerical models was found to be scalable and acceptable for resolving the necessary detail in the BSC.

The sintering behavior of a ceramic is best characterized by dilatometry that measures the change in length of a material as it is heated under a controlled atmosphere. By measuring the sintering behavior of material pressed under identical conditions sintered over a range of CHRs, a family of sintering curves is generated for the uranium powder received from AREVA. These curves is collapsed into a Master Sintering Curve (MSC) and used to predict the sintering behavior of the material for any arbitrary sintering profile. For all compositions of the oxygen-to-metal $(\mathrm{O} / \mathrm{M})$ ratios, we find that the scatter in the MSC model fit is minimal indicating excellent reproducibility of the data. This indicates that independent of the $\mathrm{O} / \mathrm{M}$ value used for the sintering process, the sintering process is dominated by only one diffusion mechanism and the MSC theory is applicable to describing the sintering of oxide nuclear fuel pellets.

\section{Metallic-Based Fuels Technologies}

Metallic-Based Fuels Technologies produced significant achievements in all work areas despite a prolonged radiological work stand down. Eight Level 2 and 19 Level 3 milestones were completed. Some of the highlight
Analytic Support major

accomplishments include:

- BISON framework found adequate to develop analysis tools for metallic and oxide fuels. A validation database was created for $\mathbf{1 0}$ Metallic Fuel Pins from EBRII experiment $X 425$.

- A methodology was developed to evaluate the performance and safety characteristics of advanced innovative fuel concepts in LWRs.

- FLOW3D code found adequate for performing casting simulations.

- The Master Sintering Curve theory found applicable to describing the sintering of oxide nuclear fuel pellets.
Metallic-Based Fuels Technologies major accomplishments include:

- Radial profiling of thermal diffusivity at $\mathbf{5 0}$ um spatial resolution (highest known spatial resolution capability) completed on four irradiated metal fuel samples using the newly implemented STDM.

- Advanced the study of Fundamental Fission Products Behavior and Thermophysical Properties.

- Fabricated and characterized AFC-3A and AFC-3B irradiation test fuels and experiments.

- Evaluated Sb effectiveness in immobilizing the Ln fission products.

- Fabricated initial U-Mo fuel samples for metallic LWR fuels. 
accomplishments for FY 2011 include the radial profiling of thermal diffusivity at $50 \mu \mathrm{m}$ spatial resolution (highest known spatial resolution capability) on four irradiated metal fuel samples using the newly implemented Scanning Thermal Diffusivity Microscope (STDM); the installation and application of radioactive materials into the Electron Probe MicroAnalyzer (EPMA); the continuing development of the Focused Ion Beam (FIB) and Micro-focus X-ray Diffractometer ( $\mu$-XRD) to higher levels of radioactive materials; the fabrication of 60 transmission electron microscopy (TEM) samples for irradiation in the High-Flux Isotope Reactor (HFIR) rabbit system; the development of a technique for the fabrication of annular fuel forms; the fabrication and characterization of the AFC-3A and AFC-3B irradiation test fuels and experiments; the development and testing of a method for trace element analysis of Fe and Si on fuels; the completion of the fabrication of the glove-box advanced casting system (GACS); the initiation of the construction of a multifunctional prototype instrument for laser ultrasound measurements; the testing of a radio frequency/surface acoustic wave wireless transmission system for in-pile measurement applications; the initial development of a thermo-acoustic sensor for in-pile measurements; test runs of the $\mathrm{AmO}_{2}$ reduction/distillation apparatus with surrogates; the successful development and realization of a method to produce representative porous metal materials for investigations of fission product migration mechanisms; significant advancement in the fundamental studies on constituent migration and fuel-cladding-chemical-interaction (FCCI) in metal fuels.

Advanced Metallic Fuel Concept for Reliable Performance to Ultra-High Burnup. As an outcome of the AFC call for innovative fuels concepts, Argonne National Laboratory (ANL) and Idaho National Laboratory (INL) received funding to begin developing an innovative design concept for ultra-high burnup sodium-cooled fast-reactor metallic fuel. Highlights for FY 2011 include the assessment of the impacts of vented-to-coolant fuel designs on core performance, radioactivity level in the primary coolant and cover-gas space, and associated need for additional biological shielding. Also, an assessment was made of potential agents to immobilize lanthanide fission products (LA) in metallic fuel. It was focused on evaluating the effectiveness of antimony $(\mathrm{Sb})$ and on determining the amounts of $\mathrm{Sb}$ that are optimum for use in fuel samples to be irradiated in the ATR in the future. The optimum Sb/LA atom ratio appears to be about 1.2.

Uranium Alloy Metal Fuel for Light Water Reactors. This project is developing a new, high density, uranium alloy metal fuel for LWRs. In FY 2011, samples were fabricated for the LWR U-Mo test and the preliminary design of the ATR U-Mo test was completed along with the reactor physics analysis.

\section{Ceramic-Based Fuels Technologies}

Ceramic-Based Fuels Technologies focused on the fundamental understanding of oxide fuels. Priority was on LWR fuels but some effort specifically on transmutation fuels continued. Key challenges include the development of reliable, low-loss fuel fabrication methods and fuel technology development to enable major increases in fuel burn up and performance (reliability, power and safety) beyond current technologies. Activities fell within the following R\&D categories: Ceramic process modeling (sintering); U-based fuel design, development and testing (International); mixed oxide (MOX) fuel processing and properties (International); and technique development and reference materials.

Some of the specific accomplishments include a Master Sintering Curve for baseline $\mathrm{UO}_{2}$ feedstock; synthesized feedstocks by MDD process for first Separate Effects Test fabrications; archived fabrication "as-built" for AFC-1A/E and
Ceramic-Based Fuels Technologies major accomplishments include:

- Established Master Sintering Curve for baseline $\mathrm{UO}_{2}$ feedstock.

- Demonstrated the ability to dynamically control $\mathrm{O} / \mathrm{M}$ during sintering.

- Fabricated initial set of urania samples in preparation for the first ceramic rabbit test in HFIR.

- Synthesis of reference single crystals of urania and urania compounds was advanced for skull melting and via exaggerated grain growth.

- Evaluated Xe gettering using $p d$. 
AFC-1G tests; completed flow sheet for co-precipitated feedstock; demonstrated ability to dynamically control $\mathrm{O} / \mathrm{M}$ during sintering and property measurements; determined the evolution of thermal diffusivity during sintering for $\mathrm{UO}_{2}$-based reference materials including materials fabricated from MDD feedstock; demonstrated synthesis of Xe-bearing Urania films by the ion beam assisted deposition technique on $\mathrm{UO}_{2}$ substrate for fission gas (fission gas) studies; and completed design and testing of cold crucible for urania and thoria crystal growth via the skull melting technique.

The Vented Fuel Pellet/Getter Concept for High Burnup Fuel. Experiments were conducted with candidate getter materials in an environment employing a new standardized temperature vs. time profile and a reconfiguration of the TGA to ensure a much higher Xe concentration in the adsorbate mixture. Previous work had been with a mixture of $\mathrm{He}$ and Xe. The results show very favorable adsorption of Xe by Pd powder, which may be due to chemisorption or a phase change in the Pd. The surface area of the Pd makes a very large difference in the adsorption of Xe. With the higher percentage of Xe in the adsorbate mixture the results for the carbon samples improved greatly.

\section{Coated Particle Fuels Technologies}

Coated Particle Fuels Technologies focused on the application of coated particle fuel (CPF) technology for advanced reactor platforms including LWR applications. It is highly integrated with direct modeling, fuel fabrication, irradiation testing, and post irradiation performance evaluation. FY 2011 focus areas included modeling, TRU TRISO Development, LWR Microencapsulated fuel development, and the testing of advanced clad and fuel materials being developed for enhanced safety under the FCRD and other programs.

Some of the specific accomplishments include the fabrication and assembly of a new coating furnace for use in fabricating microencapsulated fuel particles and the fabrication of the first uranium bearing Fully Ceramic Microencapsulated (FCM) fuel. This includes an intensive program for optimizing the matrix for this fuel system. FCM originally designed for uranium-burn or TRU deep-burn in LWRs, was analyzed for use in fully loaded LWR Cores.

An irradiation vehicle was designed for HFIR irradiation of fueled and surrogate FCM fuel in contact with zircaloy cladding to simulate LWR application. Metal Matrix Microencapsulated (M3) fuel was designed for increasing uranium utilization in LWRs. Fuel rods were fabricated with surrogate TRISO particles with various most outer layer coatings of pyrolytic carbon (PyC), silicon carbide ( $\mathrm{SiC})$, and zirconium carbide $(\mathrm{ZrC})$.

Coated Particle Fuels Technologies major accomplishments include:

- Fabricated the first uranium

bearing Fully Ceramic

Microencapsulated Fuel (FCM) and carried out intensive program for optimizing the matrix for this fuel system.

- Metal Matrix Microencapsulated (M3) fuel was designed for increasing uranium utilization in LWRs.

- Conducted accident testing on FCM Fuel and Advanced Fuel and Cladding Materials.

Irradiation of a series of FCM fuel pellets clad in Zircaloy-4 and M3 fuel rods (containing TRISO particles with surrogate kernels) was started in HFIR. All the fuel specimens conformed to the exact geometry of the Westinghouse $17 \times 17$ PWR bundles. First round of PIE has begun with encouraging results as to the integrity of the fuel matrix under neutron irradiation.

The assembly and demonstration was completed of a Focused Ion Beam and Simultaneous Thermal Analyzer for use on fuels for the wider FCRD program.

A process was developed and demonstrated to add $\mathrm{SiC}$ to the $\mathrm{UO}_{2}$ kernel. This milestone is on a path to verify a FY 2010 modeling milestone, which proposed this route to mitigating high burnup kernel migration, a long-standing issue for microencapsulated fuels. Incorporated $\mathrm{SiC}$ and graphite matrix 
physical properties into FRAPCON and performed a preliminary analysis to assess fuel temperatures under LWR operating conditions.

Accident Testing was conducted on FCM Fuel and Advanced Fuel and Cladding Materials. Two sets of tests were performed to provide insight into the performance of fuel/cladding materials under severe accident conditions (high temperature steam attack). A side-by-side comparison of different classes of materials is being developed through examination of a wide matrix of materials including zirconium alloys, $\mathrm{SiC}$ components, advanced fuel forms, as well as conventional and advanced ferritic and austenitic steels.

\section{Gore Materials Technologies}

Core Materials Technologies focused on fast reactor clad materials for high dose applications in the following R\&D areas: Knowledge base development for High Dose (up to $200 \mathrm{dpa}$ ) Core Materials Irradiation Data (ACO-3 duct testing and Fast Flux Test Facility [FFTF]/Materials Open Test Assembly [MOTA] testing) and Advanced Material Development (advanced cladding materials and coatings/liners to mitigate FCCI).

The analysis of specimens from the ACO-3 Duct involved analysis of Charpy, compact tension, tensile and TEM specimens. A detailed microstructural analysis was performed of the phase distribution along the duct (TEM and Atom Probe analysis). Rate jump testing was performed to provide detailed data for model development. Thermal annealing was performed at 550 and $650^{\circ} \mathrm{C}$ followed by fracture toughness testing at Oak Ridge National Laboratory (ORNL).

For innovative liner development, the design of AFC-3 materials capsule was completed. Modifications were necessary to reach desired temperature. The first cycle capsule will only include cladding-fuel-liner diffusion couple samples. Out of Pile cladding-fuel-liner and coating DC studies are ongoing at Texas A\&M University (TAMU).

Coatings were fabricated on HT-9 and MA957 and underwent Fe heavy-ion irradiation at $500^{\circ} \mathrm{C}$. Cross-sections of the coatings were prepared in the FIB and analyzed by FEG-STEM.

Core Materials Technologies major accomplishments include:

- Completed the design of AFC-3 materials capsule for innovative liner development.

- Fabricated coatings on HT-9 and MA957, which underwent Fe heavy-ion irradiation at $500^{\circ} \mathrm{C}$. Cross-sections of the coatings were prepared in the FIB and analyzed by FEG-STEM.

- A large heat (>50 kg) of ODS strengthened ferritic steel (14YWT) was produced and bars were consolidated using HIP'ing and hot extrusion followed by forging and rolling processing.

Innovative processing for oxide dispersion strengthened ODS alloys included the fabrication of Friction Consolidation and Extrusion (FCE) dies and the initiation of the experimental work to establish process parameters for densifying powder metal ODS alloy precursors.

A large heat ( $>50 \mathrm{~kg}$ ) of ODS strengthened ferritic steel (14YWT) was produced and bars were consolidated using hot isostatic pressing and hot extrusion followed by forging and rolling processing. Mechanical testing and irradiation testing of this material is underway. First data was obtained on an ODS steel, (MA-957) after irradiation in FFTF to doses up to $100 \mathrm{dpa}$. This is the highest dose data reported on this ODS steel. 


\section{Irradiation Testing Technologies}

Irradiation Testing Technologies focused on continued irradiation of fast reactor fuel concepts in ATR, preparations for separate effects testing (SET) using the hydraulic rabbit facility in HFIR, and PIEs of fuels irradiated in ATR, EBR-II and FFTF. Some of the specific accomplishments include the continued irradiation of AFC-2D High Burnup MA-MOX fuel test and AFC-2E Metallic Fuel Fabrication Variables test in ATR. The insertion of AFC-3A, B experiments in ATR to demonstrate feasibility of the Advanced Metallic Fuel Concept for Ultra-high Burnup. In addition, progress was made in the design of AFC-4 Instrumented Lead Experiment for SET Testing in ATR, along with R\&D on advanced sensors for in situ measurements of irradiation performance parameters.
Irradiation Testing Technologies major accomplishments include:

- Inserted AFC-3A,B experiments in ATR to demonstrate feasibility of the Advanced Metallic Fuel Concept for Ultra-high Burnup.

- Obtained DOE safety approval for fuel testing in HFIR hydraulic rabbit facility, to begin in October 2011.

- First PIE results on MA-MOX fuels irradiated in ATR showed classic restructuring pattern, validating Cd-shrouded testing concept in ATR.

DOE safety approval was obtained for fuel testing in HFIR hydraulic rabbit facility, to begin in October 2011. First metallic fueled rabbit capsules were fabricated at INL/ORNL and were ready for testing at year end.

PIE was completed on AFC-1D, 1G, 1H, 2A experiments from ATR and the PIE Report was issued. The first PIE results on MA-MOX fuels irradiated in ATR showed classic restructuring pattern, validating Cd-shrouded testing concept in ATR. PIE on legacy FFTF and EBR-II metallic and oxide fuels was initiated. 


\section{BONTENTS}

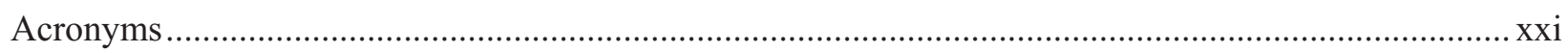

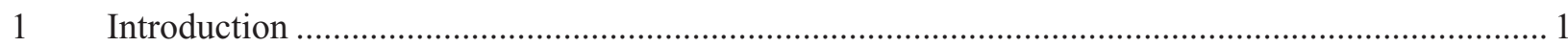

2 Campaign Management and Integration..................................................................................... 1

2.1 Advanced PIE Capabilities National Workshop ….......................................................... 1

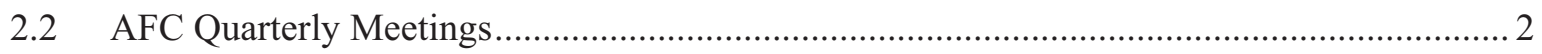

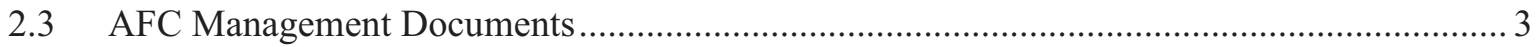

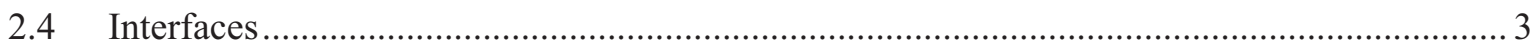

2.4.1 Advanced Light Water Reactor Interface .................................................... 3

2.4.2 Fuels-Separations-Waste Forms Interface .................................................. 4

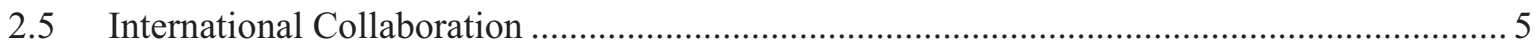

2.5.1 Republic of Korea CRADA and INERI Projects .................................................. 5

2.5.2 International Workshop on PIE Needs ................................................................. 5

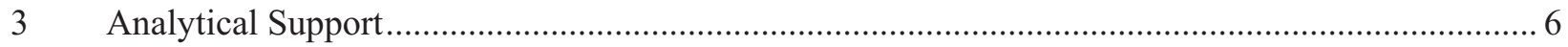

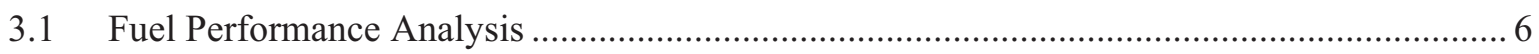

3.1.1 Fuels Performance Analysis Requirements ....................................................... 6

3.1.2 Assessment of BISON Fuel Performance Code to Develop a Metallic Fuel Analysis Tool (BISON-METAL) ........................................................................ 7

3.1.3 Metal Fuel Validation Data .......................................................................... 9

3.2 Metal Fuel Performance Sensitivity Assessment .............................................................. 9

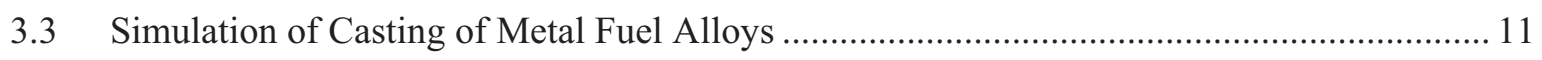

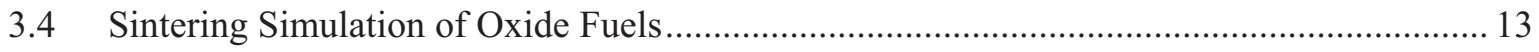

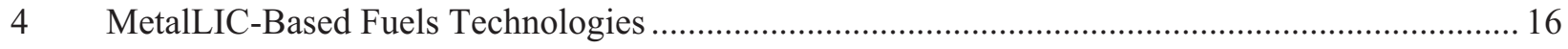

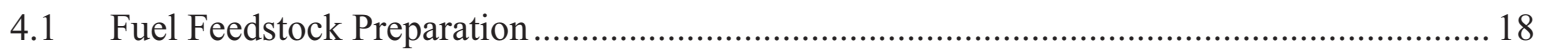

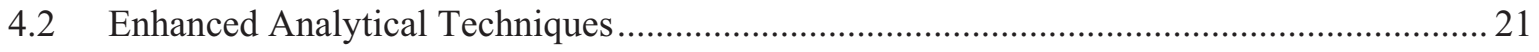

4.3 Fabrication Technique Development .............................................................................. 22

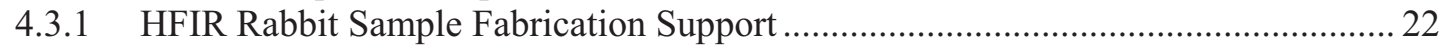

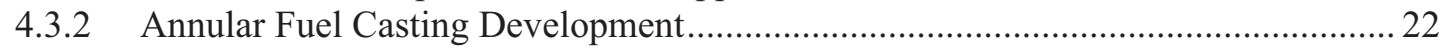

4.3.3 Glovebox Advanced Casting System Development and Testing............................. 26

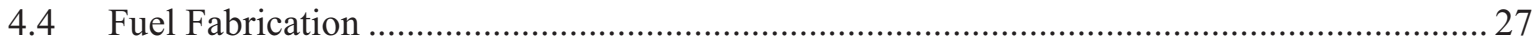

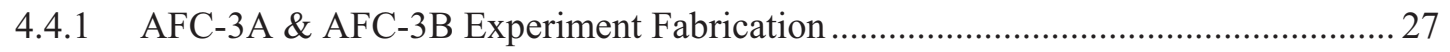

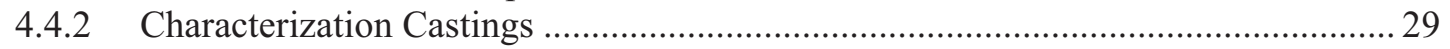

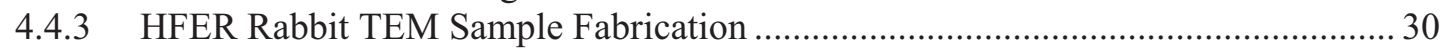

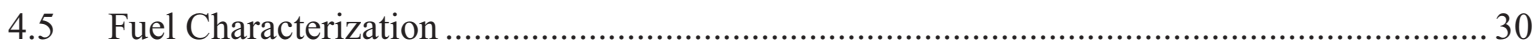

4.5.1 Scanning Thermal Diffusivity Microscope ……................................................... 37

4.6 Fundamental Fission Products Behavior.............................................................................. 38

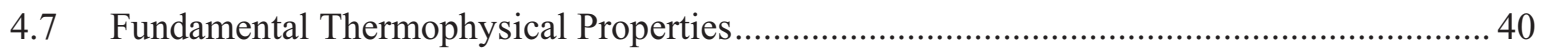

4.8 Fundamental Element Redistribution Studies .................................................................. 42

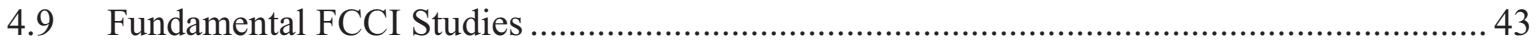

4.10 Characterization Technique Development ...................................................................... 47 


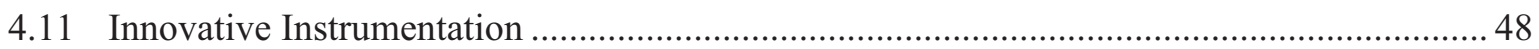

4.12 Advanced Metallic Fuel for Fast Reactors - INL .............................................................. 49

4.13 Advanced Metallic Fuel for Fast Reactors- ANL …........................................................52

4.13.1 Ultra-High Burnup Reactor Core Concept............................................................ 53

4.13.2 Thermo-Mechanical Modeling of Advanced Metallic Fuels ................................... 55

4.13.3 Atomistic Simulations of the Effect of Alloying Additions to U-Zr Fuel for

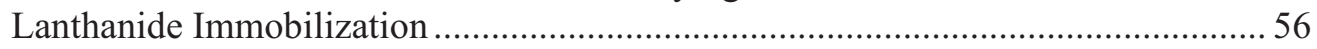

4.13.4 Out-Of-Pile Experiment on Targeted Fuel Alloy Addition .......................................58

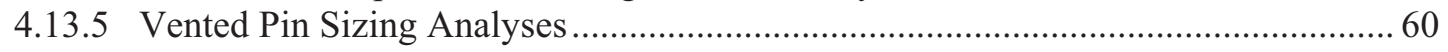

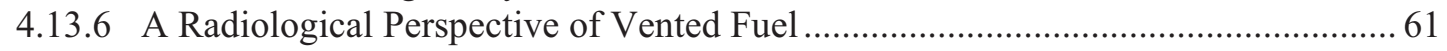

4.14 Advanced Metallic Fuel for Light Water Reactor - PNNL ................................................. 62

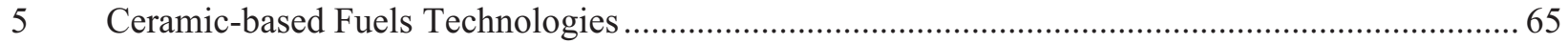

5.1 Processing of Uranium Oxide Powders Synthesized by Advanced Techniques................... 66

5.2 Microstructural Control of Uranium Oxide Based Materials................................................. 68

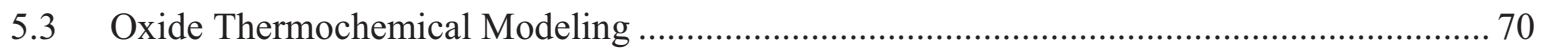

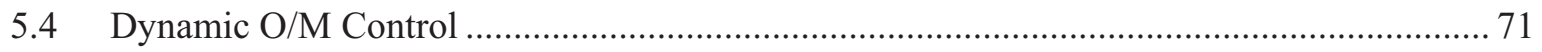

5.5 Sintering Modeling to Support Process Fabrication ........................................................ 72

5.6 Microstructural Characteristics of Sintered Urania............................................................. 73

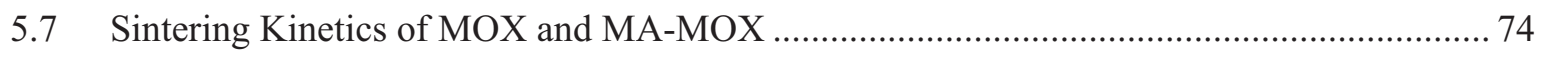

5.8 In Situ Laser Flash Analysis of Ceramic Fuel Compacts During Sintering.......................... 75

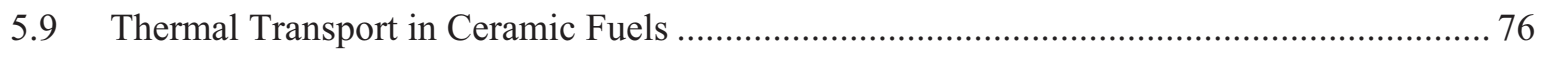

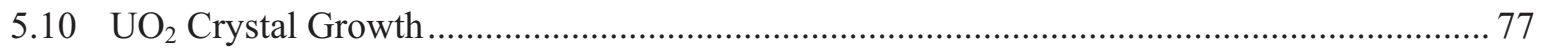

5.11 Uranium Dioxide Films with Embedded Xenon ............................................................. 78

5.12 Thoria Feedstock Procurement and Authorization of Experimental Operations .................. 80

5.13 Vented Fuel/Getter Concept..................................................................................... 81

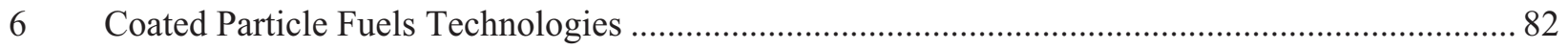

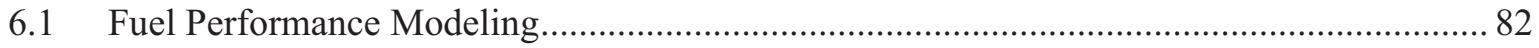

6.2 Thermochemical Data and Model Development ........................................................... 85

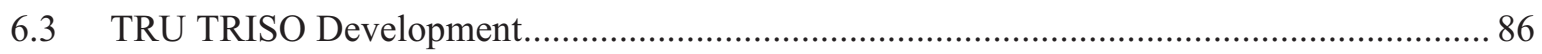

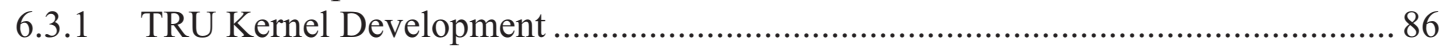

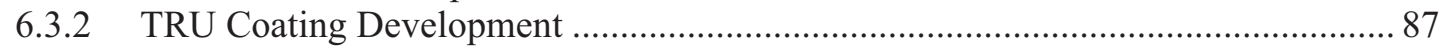

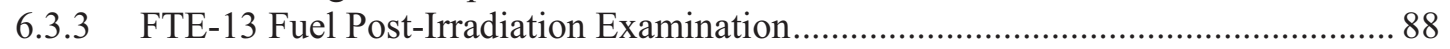

6.4 Light Water Reactor Fully Ceramic Fuel Development ................................................. 89

6.4.1 FCM Fuel Fabrication with Surrogate TRISO Particles and Preparation of Surrogate FCM Pellets for HFIR irradiation........................................................... 89

6.4.2 Accident Testing of Current and Advanced Fuel and Cladding Materials ............... 90

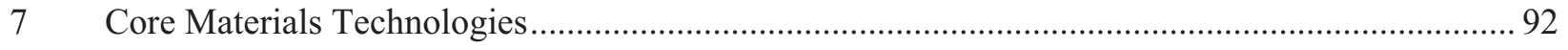

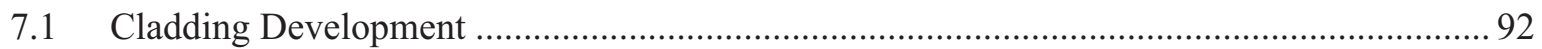

7.1.1 HT-9 Knowledge- based Development to Very High Doses (>200 dpa) ................. 92

7.1.2 Effect of Thermal Annealing on Fracture Toughness in HT9 ..................................... 94

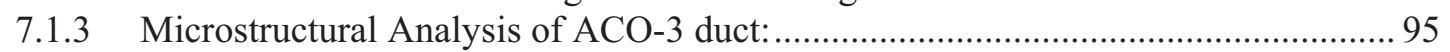

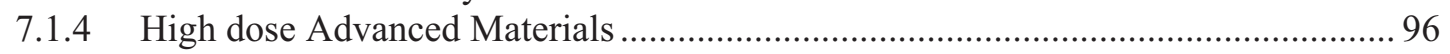




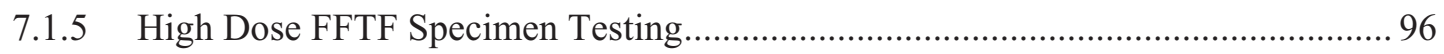

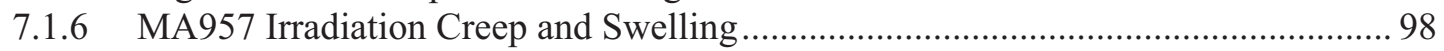

7.1.7 Coating and Liner Development for FCCI Mitigation........................................... 99

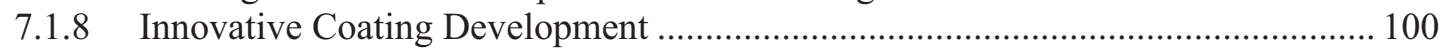

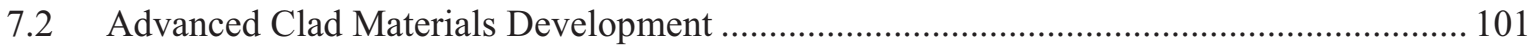

7.2.1 Development of Large Scale Heat of an Advanced ODS Alloy .............................. 101

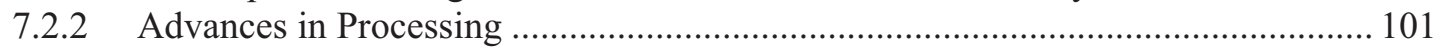

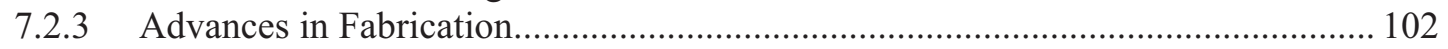

7.2.4 High Temperature Mechanical Characterization of ODS Alloys ............................. 103

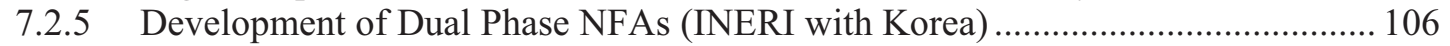

7.2.6 Innovative Tube Processing for ODS Steels ......................................................... 107

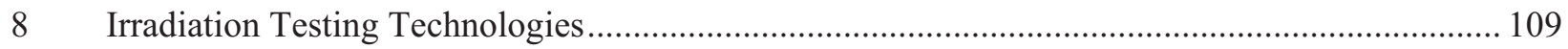

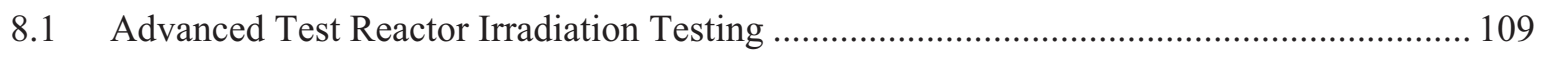

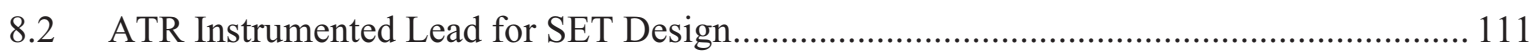

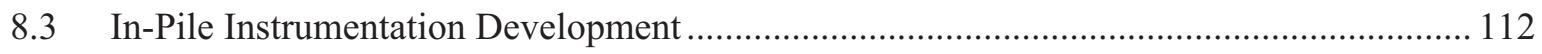

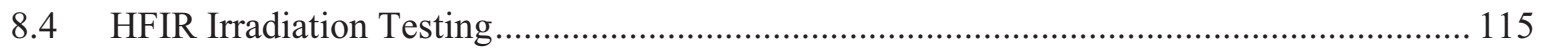

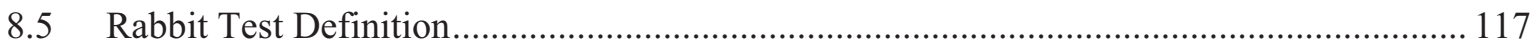

8.6 Post Irradiation Examinations ..................................................................................... 118

\section{FIGURES}

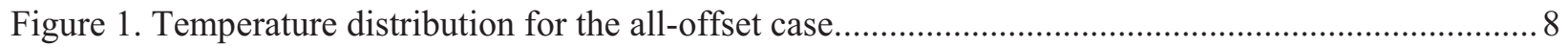

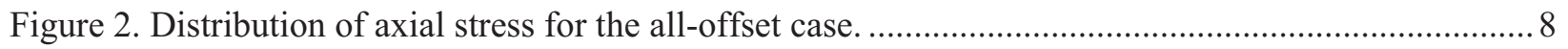

Figure 3. The shape of the cladding for the all-offset case. (Displacement is magnified by 100 times. The values of cladding-capsule radial gap are shown for each location marked with a black dot. The '-' sign signifies that the contact has not occurred, and the

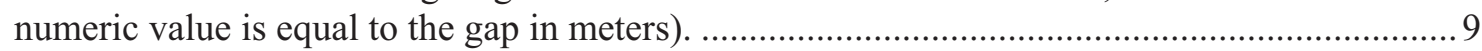

Figure 4. Quadrant of 17x17 PWR assembly with UOX rods (gray) and TRISO-TRU rods (red)........... 10

Figure 5. Temperature distribution within a TRISO particle at $0.509 \mathrm{sec}$. assuming a mid-range estimate for the specific heat.

Figure 6. Snapshots from filling with vent only at the rod bottom .

Figure 7. Final Simulation state for four venting scenarios comparing model with and without surface tension. The model without surface tension is represented by the left image in each case while the model including surface tension is represented by the right image. Including surface tension when the mold is initially occupied by argon makes significant differences in the simulation results. Appropriate venting at multiple locations in the mold will help to remedy the problem of argon becoming trapped between different sections of the U-10wt $\% \mathrm{Zr}$.

Figure 8. Result of BSC casting with argon initially in the mold. Each rod cavity is 10 inches

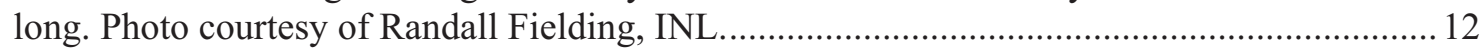

Figure 9. SEM image of as-received depleted uranium oxide from AREVA.......................................... 14

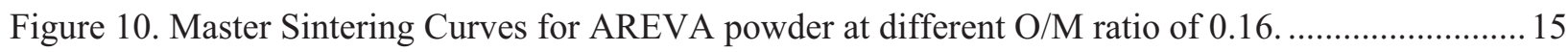

FCRD-FUEL-2012-000025 xiii 
Figure 11. (a) $\mathrm{Np}$ metal and molten salt in bottom of $\mathrm{MgO}$ crucible, (b) removed, (c) hand mill for crushing to remove $\mathrm{Np}$ "nugget" for analysis or further purification.

Figure 12. (a) Am-Np feedstock, (b) hand mill crushing, c) condensed Am (ITU), (d) drawing of small scale Am distillation apparatus with gamma detector, and (e) actual gamma detector (head).

Figure 13. Details of the Am distillation/condensation apparatus: (a) heating element, tantalum tube, and molten metal, (b) Cu cold finger (gold color) with heat shield and cap, and (c) Ta deposition cap (removable).

Figure 14. (a) Cold finger with Ta tip being inserted into Ta distillation tube, (b) outer end of long copper cold finger, (c) high temperature $\left(1600^{\circ} \mathrm{C}\right)$ furnace, and (d) Mn metal obtained from a test run.

Figure 15. Typical example of cast product for TEM disc preparation (scale is in inches)......................22

Figure 16. Quartz mold used for arc casting of annular pins............................................................. 23

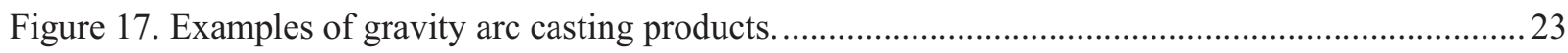

Figure 18. Examples of casting results using a Vacuum Pressure Casting Machine................................2 24

Figure 19. Typical examples of the "straws" cast without use of an inner core. ......................................2 25

Figure 20. Equipment, molds, and example results from the CGIC process development studies. ...........25

Figure 21. Scanning electron micrograph of the U-Zr-Pd alloy microstructure formed from the CGIC process. .26

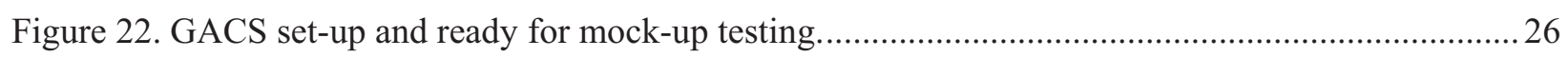

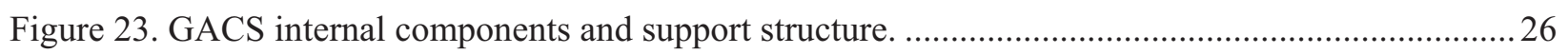

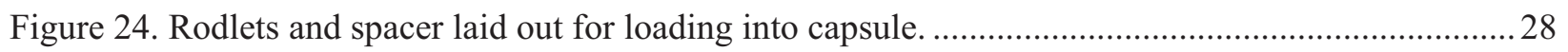

Figure 25. Verifying rodlet serial numbers and inserting rodlet into capsule........................................28

Figure 26. Orbital welding system used for capsule closure weld inside a He glove bag with a test

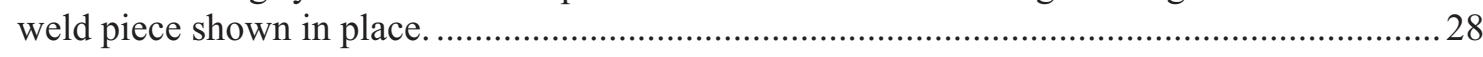

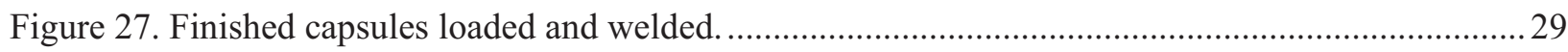

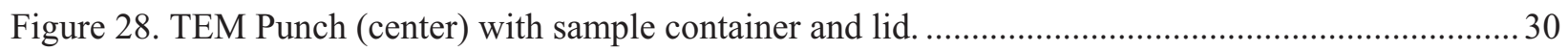

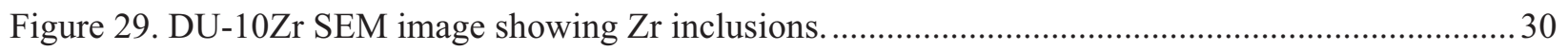

Figure 30. New instrumentation installed in the Fuels and Applied Science Building (FASB): (a) DSC, (b) LFD instrument, and (c) Dilatometer.

Figure 31. Back scattered electron image (upper left) and X-ray maps showing the microstructure with $\mathrm{Zr}$ and $\mathrm{Pd}$ co-located in inclusions.

Figure 32. Secondary electron images showing locations from which lamellae were obtained (circles with $+\mathrm{s}$ in the center indicating lamellae locations). (a) The U,Pu-Mo diffusion couple with the U,Pu shown as the lighter region to the left and Mo as the uniform gray region to the right. (b) The $\mathrm{U}, \mathrm{Pu}-\mathrm{Zr}$ diffusion couple with the $\mathrm{U}, \mathrm{Pu}$ shown as the lighter region to the right and $\mathrm{Zr}$ as the uniform dark region to the left. 
Figure 33. Secondary electron images showing lamellae from (a) position 2 in the U,Pu-Mo diffusion couple (Figure 32a), and (b) position 4 in the U,Pu-Zr diffusion couple (Figure 32b).

Figure 34. Secondary electron images showing mounted lamellae ready for insertion in the TEM. The lamellae are the small, light colored rectangular objects welded to the tops of the posts above the large letters. (a) Lamallae from the U,Pu-Mo diffusion couple, and (b) lamellae from the U,Pu-Zr diffusion couple. The numbers at the bottoms of the posts correspond to the positions indicated in Figure 32 from which the lamellae were obtained.

Figure 35. BSE images from as-cast $(\mathrm{a}, \mathrm{c})$ and annealed $(\mathrm{b}, \mathrm{d})$ samples of a Pu-10Zr sample (AFC2-B5). Numbers and arrows in a) show what may be four phases. Linear features in (c) are scratches from sample preparation.

Figure 36. BSE images from as-cast (a, c, e) and annealed (b, d, f) samples of a Pu-30Zr alloy.

Figure 37. BSE showing details of a high-Zr dendritic inclusion in an as-cast $\mathrm{Pu}-30 \mathrm{Zr}$ alloy. The finely speckled appearance of the interior indicates a nanocrystalline mixture of two phases with significantly different atomic numbers. 36

Figure 38. Optical image of the irradiated fuel sample (image courtesy of Heather Chichester).............. 38

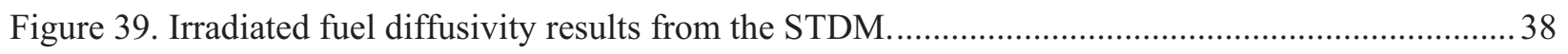

Figure 40. Illustration of possible transport rates in relation to mechanism and porosity........................ 39

Figure 41. Porous U-Zr pellet produced with approximately $20-30 \%$ porosity, by image analysis........... 39

Figure 42. The PPMS at UNLV. During measurement, the sample resides in the dark gray Dewar near the center of the photograph.

Figure 43. Measured heat capacity of uranium compared to the measured heat capacity of uranium from a DU penetrator containing 3 at $\% \mathrm{Ti}$ as a function of temperature. The difference between the two is easily distinguished in the 100 to $400 \mathrm{~K}$ range.

Figure 44. Back scattered electron image of a sputtered tungsten coating deposited on a glass slide. The coating is the bright band across the image. The coating thickness as indicated in the inset is $846 \mathrm{~nm}$. The coating was deposited in six steps, resulting in the vague banding in the image. A crack is visible in the glass substrate below the coating where the coating pulled away (presumably when potted to produce the met mount for polishing) taking a layer of the glass slide with it. This indicates good mechanical strength and adhesion.

Figure 45. Backscatter electron micrograph and concentration profile of Mo vs. Fe diffusion couple annealed at $900^{\circ} \mathrm{C}$ for 30 days.

Figure 46. Backscatter electron micrograph of $\mathrm{Zr}$ vs. Fe diffusion couple annealed at $850^{\circ} \mathrm{C}$ for 15 days......

Figure 47. Backscatter electron micrograph and concentration profile of Mo vs. Zr diffusion couple annealed at $950^{\circ} \mathrm{C}$ for 15 days.

Figure 48. Backscatter electron micrograph and concentration profile of $U$ vs. Fe diffusion couple annealed at $650^{\circ} \mathrm{C}$ for 4 days.

Figure 49. Backscatter electron micrograph and concentration profiles of $U$ vs. Mo diffusion couple annealed at $1000^{\circ} \mathrm{C}$ for 24 hours. 


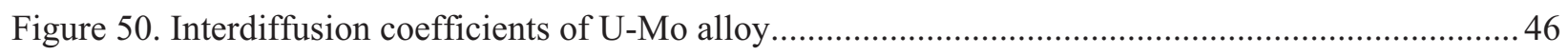

Figure 51. Temperature dependence of interdiffusion coefficients at $\mathrm{NMo}=0.12,0.18,0.24 \ldots \ldots \ldots \ldots \ldots . . .46$

Figure 52. Backscatter electron micrograph of U-10wt.Zr vs. Fe diffusion couple annealed at

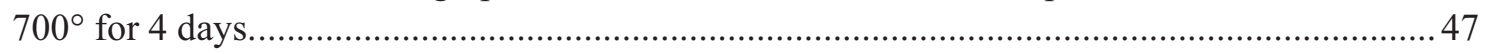

Figure 53. Arrhenius relation of growth constant for layer A, B, C, and SUM....................................... 47

Figure 54. Photograph of prototype instrument for laser ultrasound measurements. .............................. 48

Figure 55. Dot maps for energy dispersive X-ray spectrometry of U-15Zr-1.6Pd-2.2Ln. ( $\mathrm{Ln}=$ $53 \mathrm{Nd}-25 \mathrm{Ce}-16 \mathrm{Pr}-6 \mathrm{La}$, values in weight percent.) The palladium and total lanthanides are in the atomic ratio of 1-to-1, and the lanthanide content of the alloy represents approximately 8 atom percent burnup.

Figure 56. SEM of diffusion couple: $\mathrm{Nd} / \mathrm{Fe} / \mathrm{Pd}-\mathrm{Nd}$ alloy (materials, left to right) illustrating benefit of intermetallic formation between palladium and neodymium. The diffusion couple was heated at $700^{\circ} \mathrm{C}$ for 100 hours, and the double reaction zone is apparent on

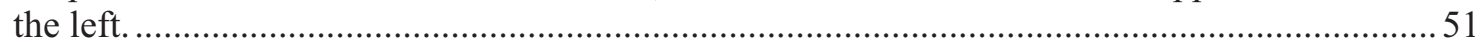

Figure 57. Homogeneous (left) and Heterogeneous (right) UHBR Core Concept ................................... 54

Figure 58. Total BFS energy (blue) of the computational cell as a function of temperature (0 to $1400 \mathrm{~K}$ ), and its strain (red) and chemical (green, lower curve) components for (a) the reference $\mathrm{U}-\mathrm{Zr}-\mathrm{Ln}$ cell (no additives) and the U-Zr-Ln cell with additions $\mathrm{X}$ for $\mathrm{X}=$ (b) In, (c) Tl, (d) Ga, (e) Sb, and (f) Pd.

Figure 59. Optical micrograph of the cross section of U-10Zr-2Ce-5In alloy sample ............................59

Figure 60. Illustration of Diving Bell Fuel Vent Concept with Relevant Dimensions Indicated ............... 60

Figure 61. Relative measures of fission-product doses after fuel burnup to 34 at $\%$................................6 62

Figure 62. Illustration of materials relationships between starting feedstock chemistry, processing conditions, resulting microstructure, associated properties and subsequent fuel performance.

Figure 63. EDS analysis of DUO2 - 20 at $\%$ Ce prepared by conventional processing after one heat treatment cycle: (A) Secondary Electron image, (B) U map, (C) Ce map, (D) O map.

Figure 64. EDS analysis of fully reduced MDD UO2 - 20at \% Ce. (A) Secondary Electron image,

(B) U map, (C) Ce map, (D) O map. ....

Figure 65. EBSD patterns of uranium oxide pellets sintered at $1350{ }^{\circ} \mathrm{C}$ (left) and $1650{ }^{\circ} \mathrm{C}$ (right). The pellets are approximately the same density but the grain size has been increased by a factor of 7 .

Figure 66. SEM image showing fine porosity induced by the addition of starch.

Figure 67. SEM image showing coarser porosity induced by the addition of starch.

Figure 68. Comparison of ORNL and Zinkevich models to literature data............................................. 70

Figure 69. $\triangle \mathrm{GO} 2$ versus $\mathrm{O}: \mathrm{M}$ from experiment compared with $\mathrm{CEF}$ model for $(\mathrm{U}, \mathrm{Ce}) \mathrm{O} 2 \pm \mathrm{x} \ldots \ldots \ldots \ldots \ldots \ldots . . . . . . .70$

Figure 70. (a) TGA curves for the sintering of urania using a dynamic gas mixing system, (b) Dynamic vs. conventional sintering of urania resulting reduced density by controlled sintering. 
Figure 71 Empirical sintering curves (a) are collapsed in to a Master Sintering Curve (b) allowing prediction of the density over any arbitrary time/temperature excursion. .................................. 73

Figure 72. Grain boundary character distribution for CSL GBs for two pellet densities..........................73

Figure 73. (a) 3D Reconstruction of the microstructure, (b) 3D GB network and (c) 3D GB................. 74

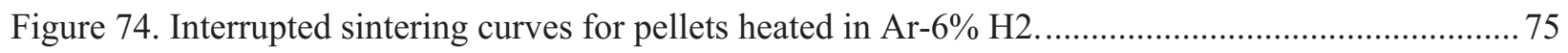

Figure 75. Dilatometry curves of pellets sintering in an Ar atmosphere. ............................................. 75

Figure 76. Results from in situ LFA sintering studies performed for UO2 heated at two different rates. The temperature scale is restricted to show only the early stages of sintering to highlight the role of in situ LFA studies compared to data generated using conventional

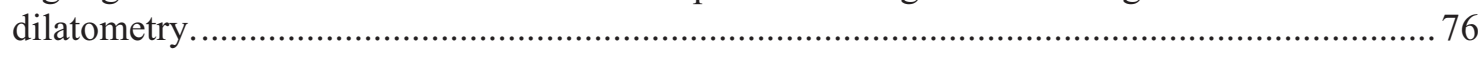

Figure 77. The thermal diffusivity of three urania-ceria solid solutions plotted against temperature. The original data is plotted on the left, while the inverse diffusivity is shown on the right. Plotting of the inverse diffusivity allows for graphical extraction of the pre-exponential factors $\mathrm{A}$ and $\mathrm{B}$.

Figure 78. (a) Illustration of skull melting process illustrating melting in the center of the powder, powder filling into melt, full melt and nucleation and growth of grains; (b) zirconia crystals formed from skull melting test.

Figure 79. Schematic of IBAD process employed for fabrication of $\mathrm{DUO}_{2}$ film doped with $\mathrm{Xe}$ atoms. Deposition of the $\mathrm{DUO}_{2}$ film is carried out by electron beam (e-beam) evaporation and $\mathrm{Xe}$ atoms are incorporated into the growing film by simultaneous bombardment with low energy Xe ion beam.

Figure 80. Photo image of the $\mathrm{DUO}_{2}$ substrate coated with a $5.1 \mu \mathrm{m}$ depleted urania film with embedded Xe (lower half of sample). The upper half of the sample, containing the crack, is the substrate without the film, which was shielded during the deposition.

Figure 81. RBS spectra obtained from the $\mathrm{UO} 2<\mathrm{Xe}>$ film deposited on silicon carbide substrate and coated with protective alumina layer. Peaks corresponding to backscattering from $\mathrm{U}$ and $\mathrm{Xe}$ atoms are labeled on the graph. Features at channel numbers below 500 correspond to backscattering from $\mathrm{Si}, \mathrm{Al}, \mathrm{O}$ and $\mathrm{C}$ atoms. We did not observe any changes in RBS spectra before and after annealing 80

Figure 82. Getter in plenum separated from fuel stack.

Figure 83. Kinetic Monte Carlo simulations of the fractional release of silver at $1700^{\circ} \mathrm{C}$ : a) influence of the reflective nature of the $\mathrm{PyC} / \mathrm{SiC}$; activation energies for particles 5, 6 and 7, respectively $(0 \mathrm{eV}, 0 \mathrm{eV}, 0 \mathrm{eV}),(0 \mathrm{eV}, 2 \mathrm{eV}, 1 \mathrm{eV})$ and $(0 \mathrm{eV}, 2 \mathrm{eV}, 2 \mathrm{eV})$; b) influence of the cavity de-trapping activation energy with a given cavity number density of $10^{18} \mathrm{~m}^{-3}$ in SiC. Particle 8 has no cavities, whereas 9, 10 and 11 have detrapping activation energies of $2.7 \mathrm{eV}, 3.0 \mathrm{eV}$ and $3.3 \mathrm{eV}$, respectively; c) influence of the grain geometry in SiC; particle 12 has no grains, 13 has square $1 \mu \mathrm{m}$ grains, 14 has rectangular grains $1 \mu \mathrm{m}$ in height and a range of width from $1 \mu \mathrm{m}$ to $40 \mu \mathrm{m}$; d) influence of the cracking within the $\mathrm{SiC}$ layer, cracks are $20 \mathrm{~nm}$ wide and provide instantaneous transport path for incident $\mathrm{Ag}$ atoms.

Figure 84. Increase in thermal resistance as a function for defect concentration for six different types of defects. 
Figure 85. The tensile stress generated within the $\mathrm{SiC}$ matrix as a result of interactions between the stress fields associated with each TRISO particle arranged in a simple cubic array increases rapidly as the inter-particle spacing decreases (i.e., particle loading increases)......... 85

Figure 86. Computed nitrogen pressure over $\mathrm{UC}_{0.5} \mathrm{~N}_{0.5}$ as a function of temperature. ............................ 86

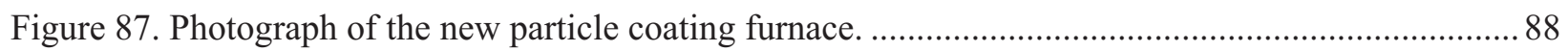

Figure 88. Left: Low-Mag SEM image of TRISI particles embedded in SiC matrix. Right: comparison of grain structure in matrix and particle's CVD-deposited SiC.

Figure 89. FCM pellets along with the zircaloy-4 tubing section, graphite spacers, and centering thimble prior to encapsulation in the HFIR rabit.

Figure 90. Steam oxidation at a) high-pressure, high-temperature b) atmospheric pressure high flow rate.

Figure 91. Optical microscopy images of sample $6 \mathrm{E} 9-6\left(\left(\mathrm{Tirr}=380^{\circ} \mathrm{C}\right.\right.$, dose $\left.=22 \mathrm{dpa}\right)$ at $160 \mathrm{x}$ multiplication. Sample had the lowest uniform, $1.27 \%$, and total elongation, $7.8 \%$, of all samples measured. Ductility is still evident, especially on sample's surface in $45^{\circ}$ image. Clockwise from top left: $2 \mathrm{D}$ image at $0^{\circ}, 3 \mathrm{D}$ multifocus image at $45^{\circ}, 3 \mathrm{D}$ image with measurements at $90^{\circ}, 3 \mathrm{D}$ image at $90^{\circ}$.

Figure 92. Rate jump experiments for HT-9 INL Control and 6E5 samples

Figure 93. Effect of $550^{\circ} \mathrm{C}$ annealing on fracture toughness in $\mathrm{HT} 9$ steel irradiated at about $379^{\circ} \mathrm{C}$ and $464^{\circ} \mathrm{C}$.

Figure 94. Effect of $650^{\circ} \mathrm{C}$ annealing on fracture toughness in $\mathrm{HT} 9$ steel irradiated at about $379^{\circ} \mathrm{C}$ and $464^{\circ} \mathrm{C}$.

Figure 95. (A) Tensile traces and, (B) tensile property plot for irradiated MA957 ODS steel.

Tensile properties of the unirradiated material are indicated by the bold "I" markers on the vertical axes in B).

Figure 96. Fracture toughness of irradiated HT-9 specimens recovered from FFTF. 98

Figure 97. Swelling estimated from diameter change of unpressurized tubes and from density change of pieces of both unpressurized and pressurized tubes. The stress of each tube measured by density is indicated in the plots.

Figure 98. Steady state creep rate of MA957 and HT-9 as a function of irradiation temperature..........

Figure 99. Schematic drawings of AFC-3 materials capsules.

Figure 100. (a) TEM image showing minimal evidence of radiation damage in coating layers after $10 \mathrm{dpa}$ at $500^{\circ} \mathrm{C}$; (b) high angle annular dark field STEM image illustrating compositional variation in coating layers and little evidence of elemental mixing.....

Figure 101. Shows the beneficial effect of using the modified ball milling condition to control the $\mathrm{O}, \mathrm{C}$ and $\mathrm{N}$ levels in the four large heats of 14YWT.....

Figure 102. Fracture toughness of the (a) 14YWT-SM11 heat and (b) 14YWT-PM2 heat in the L$\mathrm{T}$ and $\mathrm{T}-\mathrm{L}$ orientations.

Figure 103. Temperature dependence of tensile yield stress in ODS alloys and reference FM steels.

Figure 104. Temperature dependence of fracture toughness in ODS alloys and reference FM steels. 
Figure 105. The coupons cut from the extruded bars. Note that in the coupons the inner meat part is the NFA and the outer rim part is from the carbon steel can.

Figure 106. SEM BSE micrographs showing the ultra-fine grain structure of the extruded $9 \mathrm{Cr}$ NFAs: (a) 9YWTV-PM1 $(0.12 \% \mathrm{C})$ and (b) 9YWTV-PM2 $(0.05 \% \mathrm{C})$.

Figure 107. (a) Friction Consolidation and Extrusion concept and (b) Examples of plungers used to extrude rod (left) or compacted pucks (right).....

Figure 108. TEM micrographs and particle size distribution data on nanoscale dispersoids observed in FCE processed material (on left) verses material processed through traditional densification routes. The average dispersoid size in the FCE compact is $4.8 \mathrm{~nm}$, and $21.1 \mathrm{~nm}$ in the conventional product.

Figure 109. FY 2011 irradiation schedule for AFC-2 experiments.

Figure 110. Ultrasonic thermometry efforts initiated in FY 2011 include (a) identifying candidate components for optimizing UT components and (b) evaluation of commercial UTs obtained from prior INL activities.

Figure 111. Initial fiber optics investigation (a) conceptual probe design, (b) conceptual probe components, and (c) spectra recorded using conceptual probe.

Figure 112. Creep test rig positioned in HTTL autoclave for testing.

Figure 113. TEM rabbit design features the use of Gd spacers (0.9" stack length) that also serve as the thermal neutron shield. Space is provided for a maximum of 6 TEM disks samples, however $\mathrm{SiC}$ temperature monitors and flux monitors will displace some of the TEM disks as needed. The number of Gd spacers can be reduced to adjust the reactivity of the rabbit.

Figure 114. Images of the as-built TEM rabbit capsules: (a) photograph of the inner Gd stack with a $\mathrm{SiC}$ temperature monitor running the length of the stack, (b) photograph of the inner Gd stack being measured for length, (c) post-welding x-ray image of the three rabbit capsules, and d) photograph of the capsules prior to final cleaning step in preparation for irradiation.

Figure 115. AFC-2C Rodlet 3 Fuel Cross-Section $\left(\mathrm{U}_{0.75}, \mathrm{Pu}_{0.20}, \mathrm{Am}_{0.05}, \mathrm{~Np}_{0.03}\right) \mathrm{O}_{1.98}, 1.35 \mathrm{E} 21 \mathrm{f} / \mathrm{cm}^{3}$, 8.36 at.\% HM burnup 118

Figure 116. ACO-3 Fuel Cross Section 21\% FIMA Burnup. 119

Figure 117. Quantitative Maps for a MOX Fuel Pellet from the EPMA. 120

\section{TABLES}

Table 1. Key Modeling in Metal Performance Analysis Tool ............................................................... 7

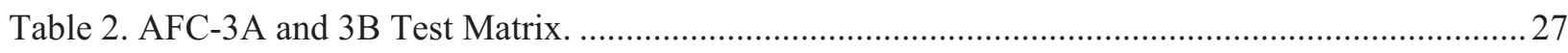

Table 3. Initial compositions and positions of irradiated fuel examined with the STDM........................ 37

Table 4. Integrated interdiffusion coefficients of $\mathrm{U}_{6} \mathrm{Fe}$ and $\mathrm{UFe}_{2}$ in $\alpha-\mathrm{U}$ and $\beta-\mathrm{U}$ terminal ends.............45

Table 5. Primary Design and Core Performance Parameters of Equilibrium UHBRs ............................. 55 
Table 6. Characteristic Temperatures (in K) of the Reference Case (U-Zr-Ln) and Different U-Zr-

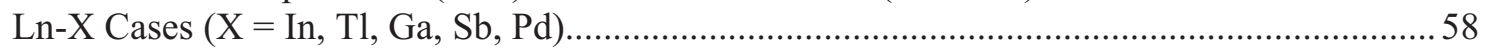

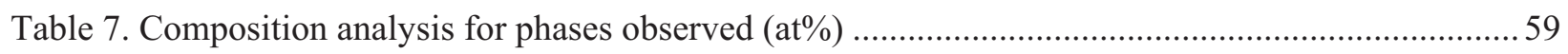

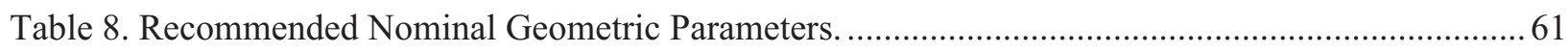

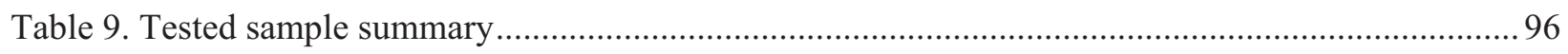

Table 10. Chemical analysis results of ball milled powder and extruded heats of 14YWT................... 102

Table 11. Test materials for uniaxial tensile and fracture toughness tests. 14YWTs and LAFs are in the NFA category; ODS Eurofer 97 and PM2000 have relatively coarse microstructures; NF616 and HT9 steels are non-ODS ferritic and/or martensitic steels.......... 104

Table 12. Cycle 149B As-Run LHGR, Fission Heat Rates, and Burnup Calculations for AFC Experiments in the EFT. 


\section{ACRONYMS}

$\mu-\mathrm{XRD}$

AFC

AFCI

ANL

APT

ASME

ASU

ATR

BFS

BNL

BOL

BSC

BSE

CAES

CALPHAD

CEA

CEF

CFN

CGIC

CHR

$\mathrm{CPF}$

CRADA

CSL

$\mathrm{CSM}$

CVD

DCT

DOE

$-\mathrm{HQ}$

-NE

-ORO

DOM

dpa

DRC

DSA

DSC

DU

EBR

EBSD

EDS
Micro-focus X-ray Diffractometer

Advanced Fuels Campaign

Advanced Fuel Cycle Initiative

Argonne National Laboratory

Atom-Probe Tomography

American Society of Mechanical Engineers

Arizona State University

Advanced Test Reactor

Bozzolo, Ferrante, and Smith Method

Brookhaven National Laboratory

beginning of life

bench-scale caster

backscattered electron

Center for Advanced Energy Studies

calculation of phase diagrams

Commissariat à l'énergie Atomique

compound energy formalism

Center for Functional Nano-Materials

counter gravity injection casting

constant heating rates

coated particle fuel

Cooperative Research and Development Agreement

Coincident Site Lattice

Colorado School of Mines

Chemical Vapor Deposition

disk compact tension

Department of Energy

Headquarters

Office of Nuclear Energy

Oak Ridge Operations Office

Digital Optical Microscope

displacements per atom

dynamic reaction cell

Documented Safety Analysis

differential scanning calorimetry

depleted uranium

Experimental Breeder Reactor

electron backscatter diffraction

energy dispersive spectroscopy 
EFPI

EFT

EML

EPMA

EPRI

FCCI

FCE

FCM

FCMI

FCRD

FE

FEG-STEM

FE-TEM

FFTF

FIB

FM

FY

GACS

GB

GE

Gen-IV

GFR

HF

HFEF

HFIR

HIP

HTGR

HTR

IAEA

IBAD

ICP-AES

ICP-MS

IFE/HRP

INERI

INL

ITU

JAEA

JSME

KAERI

KMC
Extrinsic Fabry-Perot Interferometer

East Flux Trap

Electron Microscopy Laboratory

Electron Probe Micro-Analyzer

Electric Power Research Institute

fuel-cladding-chemical interaction

Friction Consolidation and Extrusion

Fully Ceramic Microencapsulated (fuel)

fuel-clad-mechanical-interaction

Fuel Cycle Research and Development

finite element

field emission gun-based-scanning transmission electron microscope

field emission-transmission electron microsope

Fast Flux Test Facility

focused ion beam

ferritic-martensitic

fiscal year

glove-box advanced casting system

grain boundary

General Electric

Generation IV

gas-cooled fast reactor

hydrofluoric

Hot Fuel Examination Facility

High-Flux Isotope Reactor

hot isostatic pressing

High-Temperature Gas-Cooled Reactor

High-Temperature Reactor

International Atomic Energy Agency

ion beam assisted deposition

Inductively Coupled Plasm-Atomic Emission Spectroscopy

Inductively Coupled Plasm-Mass Spectrometry

Institute for Energy Technology at the Halden Reactor Project

International Nuclear Energy Research Initiative

Idaho National Laboratory

Institute for Transuranium Elements

Japan Atomic Energy Agency

Japan Society Mechanical Engineering

Korea Atomic Energy Research Institute

kinetic Monte Carlo 
LA

LANL

LFA

LFD

LHGR

Ln

LOCA

LWR

M3

MA-MOX

MAX

MDD

MFC

$\mathrm{MOC}$

MOOSE

MOTA

MOX

MSC

NEA

NEAMS

NFA

NRC

NSLS

$\mathrm{O} / \mathrm{M}$

ODS

OECD

ORNL

PEI

PIE

PNNL

PPMS

PWR

PyC

QA

R\&D

RBS

RD\&D

RE

REA

RFID lanthanide

Los Alamos National Laboratory

laser flash analysis

Laser Flash Diffusivity

linear heat generation rate

a $53 \mathrm{Nd}-25 \mathrm{Ce}-16 \mathrm{Pr}-6 \mathrm{La}$ compositional relationship

loss-of-coolant accident

light water reactor

Metal Matrix Microencapsulated (fuel)

minor actinide-bearing mixed oxide (fuel)

$\mathrm{M}_{\mathrm{n}+1} \mathrm{AX}_{\mathrm{n}}$ (Class of layered machinable ceramics)

Modified Direct Denitration

Materials and Fuels Complex

modified-open fuel cycle

Multiscale Object-Oriented Simulated Environment

Materials Open Test Assembly

mixed oxide

Master Sintering Curve

Nuclear Energy Agency

Nuclear Energy Advanced Modeling and Simulation

nanostructured ferritic alloy

Nuclear Regulatory Commission

National Synchrotron Light Source

oxygen-to-metal

oxide dispersion strengthened

Organization for Economic Cooperation and Development

Oak Ridge National Laboratory

cationic polyelectrolyte

postirradiation examination

Pacific Northwest National Laboratory

Physical Properties Measurement System

pressurized-water reactor

pyrolytic carbon

Quality Assurance

research and development

Rutherford Backscattering Spectroscopy

research, development and demonstration

rare earth

rod ejection accident

radio frequency identification 
SANS

SCS

SEM

SET

SFR

$\mathrm{SiC}$

STDM

STIP-IV

TAC

TAMU

TBP

TD

TEM

TPB

TRISO

TRU

UCSB

UCF

UNF

UNLV

UOX

UT

UTS

WBS

WDS

XRD

YS

$\mathrm{ZrC}$

Periodic Elements

$\mathrm{Ag}$

$\mathrm{Al}$

Am

C

$\mathrm{Cd}$

$\mathrm{Ce}$

$\mathrm{Cr}$

$\mathrm{Fe}$

Fs

$\mathrm{Ga}$ small angle neutron scattering

Shutdown Cooling System

scanning electron microscopy

Separate Effects Testing

Sodium-cooled Fast Reactor

silicon carbide

scanning thermal diffusivity microscope

SINQ Target Irradiation Program

thermoacoustic

Texas A\&M University

tri-butyl phosphate

theoretical tensity

transmission electron microscopy

three-point bend

tristructural isotropic

transuranic

University of California Santa Barbara

University of Central Florida

used nuclear fuel

University of Nevada-Las Vegas

uranium oxide

ultrasonic thermometer

ultimate tensile strength

work breakdown structure

wavelength dispersive spectroscopy

$\mathrm{x}$-ray diffraction

yield stress

zirconium carbide

silver

aluminum

americium

carbon

cadmium

cerium

chromium

iron

fissium

gallium 


$\begin{array}{ll}\mathrm{Gd} & \text { gadolinium } \\ \mathrm{He} & \text { helium } \\ \mathrm{In} & \text { indium } \\ \mathrm{La} & \text { lanthanum } \\ \mathrm{Mg} & \text { magnesium } \\ \mathrm{Mn} & \text { manganese } \\ \mathrm{Mo} & \text { molybdenum } \\ \mathrm{N} & \text { nitrogen } \\ \mathrm{Nb} & \text { niobium } \\ \mathrm{Nd} & \text { neodymium } \\ \mathrm{No} & \text { nobelium } \\ \mathrm{Np} & \text { neptunium } \\ \mathrm{Pd} & \text { palladium } \\ \mathrm{Pr} & \text { praseodymium } \\ \mathrm{Pu} & \text { plutonium } \\ \mathrm{Sb} & \text { antimony } \\ \mathrm{Si} & \text { silicon } \\ \mathrm{Sm} & \text { samarium } \\ \mathrm{Tl} & \text { thallium } \\ \mathrm{U} & \text { uranium } \\ \mathrm{Xe} & \text { zenon } \\ \mathrm{Zr} & \text { zirconium } \\ & \end{array}$


Intentionally left Blank 


\title{
Advanced Fuels Campaign FY 2011 Accomplishments Report
}

1 INTRODUGTION

One of the major research and development (R\&D) areas under the Fuel Cycle Research and Development (FCRD) program is advanced fuels development. The Advanced Fuels Campaign (AFC) has the responsibility to develop advanced fuels and associated technologies for the Department of Energy (DOE) using a science-based approach outlined in the DOE Office of Nuclear Energy (DOE-NE) Roadmap $^{1}$ and the Draft R\&D Objective 3 Implementation Plan. ${ }^{2}$

Accomplishments made during fiscal year (FY) 2011 are highlighted in this report, which focuses on completed work and results. The process details leading up to the results are not included; however, the technical contact is provided for each section. The order of the accomplishments in this report is consistent with the AFC work breakdown structure (WBS).

\section{References}

1. Nuclear Energy Research and Development Roadmap, Report to Congress, U.S. DOE Office of Nuclear Energy, April 2010.

2. Nuclear Energy Draft R\&D Objective 3 Implementation Plan, U.S. DOE Office of Nuclar Energy, October 2010.

\section{GAMPAIGN MANAGEMENT AND INTEGRATION}

\author{
K. Pasamehmetoglu, kemal.pasamehmetoglu@inl.gov, INL
}

An important element of the AFC is the management, integration, and coordination of the major R\&D activities across multiple national laboratories and universities working to achieve this goal. Several management activities were accomplished in FY 2011 that supports the overall goals of the FCRD program. AFC interfaces and collaborates with the FCRD campaigns, industry, various DOE programs and laboratories, federal agencies (e.g., Nuclear Regulatory Commission [NRC]), and International Organizations.

\subsection{Advanced PIE Capabilities National Workshop}

\author{
L.Braase, lori.braase@inl.gov, INL
}

A National Postirradiation Examination (PIE) Workshop ${ }^{1}$ was held March 29-30, 2011, in Gaithersburg, MD, to identify the nation's needs for PIE to support advanced fuels and nuclear material development.

During the workshop, the National PIE needs were identified from various perspectives, including universities, industry, vendors, NRC, Advanced Test Reactor (ATR) National Scientific User Facility (NSUF), and DOE-NE national laboratories. Achieving the state-of-the art capabilities that can support a complete transition to science-based approach requires progressive set of actions:

- The first step, after multiple decades of neglect, is to update and refurbish the existing capabilities and replace the older capabilities with the newer, more accurate and more reliable set of instruments.

- The second step is to introduce advanced instruments and scientific techniques, commonly used in other applications typically with nonradioactive materials into PIE applications. The 
objective is to start characterizing radioactive samples at nano-scale to micro-scale length resolutions. Some of these instruments, when applied to radioactive materials characterization, require special facilities with very strict environmental control.

- The third step is to design and develop instruments that currently do not exist but are required to measure properties at various length and time scales in order to support a complete fundamental understanding of radioactive materials behavior. Such instruments would be mostly valuable to understand separate effect phenomenology and to support the multiphysics, multi-scale, predictive fuel performance modeling efforts. These capabilities are also likely to require new facilities with very specialized environmental control and integration among multiple techniques.

At the conclusion of the workshop, four high-level needs were identified as common themes from the five breakout teams. Those needs are summarized below:

1. Understanding material changes in the extreme nuclear environment at the nanoscale, especially for highly activated fuels and materials. Nanoscale studies have significant importance due to the mechanisms that cause materials to degrade, which occur at the nanoscale.

2. Enabling additional proficiency in experimentation and analysis through robust modeling and simulation coupled with advanced characterization.

3. Advancing the infrastructure and accessibility of physical and administrative systems to meet the needs of participating organizations. The inability to accommodate different time cycles and constraints make working and collaborating within the national laboratories challenging. This also includes the development of talent and the retention of expertise to support the research needs of the future.

In-situ instrumentation and measurements will be pursued to better examine dynamic changes to materials, microstructure, deformation, and surface effects as they occur in real time rather than the static, end-state data obtained by most current PIE methods. Also the ability to interpret the PIE data accurately requires a detailed understanding of the irradiation history and associated initial and boundary conditions.

\section{References}

1. National Postirradiation Examination Workshop Report, June 2011, Idaho National Laboratory, INL/EXT-11-21922

\subsection{AFC Quarterly Meetings}

L.Braase, lori.braase@inl.gov, INL

Three AFC quarterly meetings were conducted during FY 2011. In general, the meetings consisted of DOE-NE guidance, industry partner's needs and insights, and technical presentations that provided the status of R\&D efforts. All three meetings were integrated with other FCRD campaigns. The meeting locations and dates are listed below.

- Albuquerque, NM, December 7-9, 2010. This AFC quarterly was an integrated meeting with the Separations/Waste Forms Campaigns. A task force was created to establish integration opportunities and analyze applicable fuel cycle options.

- Atlanta, GA, March 15-17, 2011. This AFC quarterly was an integrated meeting with the Systems Analysis Campaign. Following this meeting, the Advanced Light Water Reactor (LWR) Fuels Working Group met to discuss the activities associated with the Industry Task 5 agreement with DOE. 
- Brookhaven National Laboratory (BNL), NY, July 12-14, 2011. This AFC quarterly was an integrated meeting with Advanced Fuels Modeling and Simulation. The Advanced Fuels R\&D Team at Brookhaven National Lab hosted tours of the National Synchrotron Light Source (NSLS) and Center for Functional Nano-Materials (CFN).

\title{
2.3 AFC Management Documents
}

\author{
L.Braase, lori.braase@inl.gov, INL
}

Two high-level AFC documents were prepared to reflect the management approach and year-end accomplishments.

- The Advanced Fuels Campaign Execution Plan, FCRD-FUEL-2010-000058, Revision 1, was delivered to DOE-NE on September 30, 2011. It provides the structure and management of research, development, and demonstration (RD\&D) activities within the FCRD program.

- The Advanced Fuels Campaign FY 2010 Accomplishments Report, FCRD-FUEL-2011000015, was delivered to DOE in December 2010. It provides the highlights of the results of the R\&D efforts performed during FY 2010.

\subsection{Interfaces}

\subsubsection{Advanced Light Water Reactor Interface}

M. Meyer,mitch.meyer@inl.gov, INL

The Advanced LWR Fuels Industry Group first met in November of 2010 with the objective of looking 20 years ahead to the role that advanced fuels could play in improving LWR technology in areas such as waste reduction, safety, reliability, and sustainable economics for new builds. When the group met again in March 2011, the Fukushima incident was still unfolding. During this meeting, by mutual agreement, the short-term emphasis of the program was focused on determining what could be done in the near term to improve fuel accident tolerance. Work at the DOE labs and work shared by industry partners provided a better understanding of the consequences of a long station blackout and the role of fuel in preventing fission product release and secondary effects, such as hydrogen explosions. This information helps to inform decisions that will benefit the LWR industry both in the short and long-term. Further work is required to understand how Generation III+ reactors would have responded to a prolonged station blackout, and if fuel with enhanced accident tolerance would have helped to reduce the consequence of this type of accident.

The nuclear industry views fuel with enhanced accident tolerance as highly desirable. The development and deployment of new fuels, however, is closely tied to the economics of plant operation. The cost for fuel development and deployment of a new fuel is on the order of $\$ 500 \mathrm{M}$ and occurs over a time period of approximately 20 years. Market conditions are such that deployment of new fuels requires a substantial economic incentive for both the vendor and the utility. A dialog has been established with vendors and utilities to further understand how the need for accident tolerant fuel designs and the economic viability of these new fuels are coupled. In addition, new fuels will have to be tested in existing reactors, so must be thermally and neutronically compatible with current plant designs.

The viability of having a dual-path approach, including short-term and long-term objectives, was discussed. The potential issue is that the urgent needs always win out over the strategic needs. The path forward needs to be integrated and achieve short-term benefit without sacrificing important long-term objectives.

In FY 2011, an Industry Advisory Committee was formed to advise the AFC National Technical Director on issues related to LWR fuel development. The Advisory Committee received information on 
22 advanced fuel concepts (and one fuel-related concept). After evaluating the fuel concepts against the screening criteria, the Committee selected 7 concepts for oral presentations in September. The final recommendations of the Committee are to focus research in the following areas:

- Coatings on zircaloy cladding that have the potential to reduce or delay hydrogen generation at elevated temperatures

- Silicon carbide clad concepts

- Robust fuels, such as Fully Ceramic Microencapsulated (FCM) Fuel

- High density, high thermal conductivity fuels that enable technologies with enhanced accident tolerance

- Alternative metal alloy cladding materials.

\subsubsection{Fuels-Separations-Waste Forms Interface}

E. Shaber, eric.shaber@inl.gov, and J. Law, INL

On December 8, 2010, a joint session was held with the Fuels Campaign and the Separations/Waste Form Campaign in Albuquerque, NM. The purpose of the meeting was to increase familiarity of the specific research being performed within each campaign and look for areas where we can work together and establish interfaces in these areas. Major interface areas, including fuel specification requirements, were discussed in detail.

One of the results of the joint campaign meeting was the formation of a team of DOE laboratory separations, waste forms, and fuel fabrication experts, along with Industry representatives, to evaluate a full recycle and a modified open cycle (MOC) processing example processing case. Two parallel teams, one for separations and one for fuel fabrication/manufacturing, were formed to work together to evaluate reference case separations processes and the resulting fuel manufacturing processing options. The objective of this effort is the documentation of viable fuel cycle processes for the identified example case options that identify the technologies involved, the separability and fuel fabricability, the issues, the unknowns, and the wastes/waste minimization impacts of viable separations and fuels processes.

This joint team met in Salt Lake City on August 17 and 18, 2011. In addition, Industry was invited to attend (at their own expense) and representatives from AREVA and General Electric (GE) Hitachi participated in the meeting. In this kickoff meeting, scope of the effort was discussed, example cases for evaluation (one MOC and one full recycle case) were chosen, and a path forward for the effort was established. In addition, a presentation on the FiT model, developed by the Systems Campaign, was given to the team. It was decided that the FiT model would be a good tool to use in evaluation of the example cases. The full recycle case chosen for evaluation was a tri-butyl phosphate (TBP)-based aqueous separation process in which $\mathrm{U}$ and $\mathrm{Pu}$ are coextracted and additional solvent extraction processes are utilized to separate the minor actinides from the lanthanides. The final product is a U/transuranic (TRU) feed which would be fabricated into a metal fast reactor fuel for a sodium fast reactor. Used fuel from the fast reactor would be recycled using electrochemical processing. The example case chosen for the MOC concept consists of a fluoride volatility separation process to separate $\mathrm{U}$ and $\mathrm{Pu}$, along with significant impurities, for recycle as mixed oxide (MOX) to LWRs. Following MOX recycle, the used fuel would be disposed. No effort to increase purity of the product through additional processing is included.

This task was initiated in FY 2011 but the majority of the effort will be performed and completed in FY 2012. 


\title{
2.5 International Collaboration
}

\author{
J. Carmack, jon.carmack@inl.gov, INL
}

Internationally, researchers are very active with collaborations including France, Japan, European Union, Republic of Korea (S. Korea), China, and Russian Federation. These interactions and collaborations are managed through a combination of participation in GENIV Global International Forum projects, International Nuclear Energy Research Initiative (INERI) projects, and participation in bilateral and trilateral government-to-government agreements. The major highlights for FY 2011 were the Cooperative Research and Development Agreement (CRADA) signed with South Korea for the joint development of metallic fast reactor fuels and associated technologies; and the International Workshop on PIE needs for science-based fuel development, organized jointly with the Organization for Economic Cooperation and Development (OECD)/Nuclear Energy Agency (NEA).

\subsubsection{Republic of Korea GRADA and INERI Projects}

In 2011, a new CRADA agreement was signed between the Idaho National Laboratory (INL) and the Korea Atomic Energy Research Institute (KAERI) to jointly conduct R\&D on the recycle, fabrication, and irradiation of materials irradiated in a LWR. After processing and recycling used nuclear fuel at the Fuel Conditioning Facility, new fuel pins will be fabricated at the INL and irradiated in the ATR.

Two INERI projects are also being conducted jointly between U.S. researchers and KAERI researchers. The first led by Dr. T.S. Byun focuses on advanced cladding materials development and the second led by Dr. J. Rory Kennedy focuses on advanced metal fuel fabrication processes. These activities will help facilitate joint R\&D between the United States and South Korea and it is expected that significant interactions and collaborative activities will be conducted under these projects in 2012 .

\subsubsection{International Workshop on PIE Needs}

The International Workshop on Characterization and PIE Needs to Support Science-Based Development of Innovative Fuels was held June 16-17, 2011, in Paris, France. The OECD/NEA Working Party on the Fuel Cycle (WPFC) sponsored the workshop to identify gaps in global capabilities that need to be filled to meet projected needs in the 21 st century.

First and foremost, the workshop brought nine countries and associated international organizations, together in support of common needs for nuclear fuels and materials testing, characterization, PIE, and modeling capabilities. Finland, France, Germany, Republic of Korea, Russian Federation, Sweden, Switzerland, United Kingdom, United States of America, International Atomic Energy Agency (IAEA), and the Instute for Transuranium Elements (ITU; on behalf of European Union Joint Research Centers) discussed issues and opportunities for future technical advancements and collaborations.

Second, the presentations provided a base level of understanding of current international capabilities. Three main categories were covered: (1) status of facilities and near term plans, (2) PIE needs from fuels engineering and material science perspectives, and (3) novel PIE techniques being developed to meet the needs. The International presentations provided valuable data consistent with the outcome of the National Workshop held in March 2011.

Finally, the panel discussion on 21st century PIE capabilities, created a unified approach for future collaborations. In conclusion, (1) existing capabilities are not sufficient to meet the needs of a sciencebased approach, (2) safety issues and fuels behavior during abnormal conditions will receive more focus post-Fukushima; therefore we need to adopt our techniques to those issues, and (3) International collaboration is needed in the areas of codes and standards development for the new techniques. 


\section{ANALYTIC SUPPORT}

Technical Lead: C. Unal, cu@lanl.gov, LANL

Analytic Support is a new technical area for AFC focused on the analysis of the impact of novel fuel forms on reactor operations and the development of fuel fabrication models, such as sintering, casting, etc. In addition, this is the primary interface with Nuclear Energy Advanced Modeling and Simulation (NEAMS) to identify fuels and materials data needs.

The requirements of the metal and oxide fuel performance analysis tool were developed. The BISON metallic rodlet demonstration model was developed with accurate geometrical dimensions of the components and the as-run linear heat generation rate to provide representative centerline temperature results for the FCRD irradiation experiments.

A database for 10 Metallic Fuel Pins from experiment X425 irradiated at EBR-II, containing metallic fuel elements, was generated as validation data for the BISON-METAL analysis tool. The database contains detailed operating conditions for 10 pins from this experiment at different stages of irradiation and corresponding experimental measurements of cladding strain.

Calculations were performed to evaluate the performance and safety characteristics of a proposed concept by Logos Nuclear Systems based on the utilization of tristructural isotropic (TRISO) particle fuel in selected locations in a pressurized-water reactor (PWR) assembly to burn the TRU from light-water reactor (LWR) used nuclear fuel (UNF). The TRISO particles would be in silicon carbide or graphite compact/stick that would be located in conventional Zircaloy clad.

FLOW3D simulations of the casting process of metallic fuels in the bench-scale caster (BSC) filled with compressible Argon gas was developed using FLOW3D's adiabatic bubble model. We evaluated the ability of FLOW3D to run reasonably resolved simulations of the BSC and assess the magnitude of the effects of surface tension and gas compressibility. The scalability of FLOW3D to large highly-resolved simulations was also investigated. The performance of coarse and fine FLOW3D numerical models was found to be scalable and acceptable for resolving the necessary detail in the BSC.

The sintering behavior of a ceramic is best characterized by dilatometry that measures the change in length of a material as it is heated under a controlled atmosphere. By measuring the sintering behavior of material pressed under identical conditions sintered over a range of constant heating rates (CHR), a family of sintering curves is generated for the uranium powder received from AREVA. These curves is collapsed into a Master Sintering Curve (MSC) and used to predict the sintering behavior of the material for any arbitrary sintering profile. For all compositions of the oxygen-to-metal $(\mathrm{O} / \mathrm{M})$ ratios, we find that the scatter in the MSC model fit is minimal indicating excellent reproducibility of the data. This indicates that independent of the $\mathrm{O} / \mathrm{M}$ value used for the sintering process, the sintering process is dominated by only one diffusion mechanism and the MSC theory is applicable to describing the sintering of oxide nuclear fuel pellets

\subsection{Fuel Performance Analysis}

Pavel Medvedev, ;pavel.medvedev@inl.gov, Steve Hayes, INL, Wenzhong Zhou, Paul Edelmann, Pratap Sadasivan, Cetin Unal, LANL; Abdellatif Yacout, ANL

\subsubsection{Fuels Performance Analysis Requirements}

Current metal fuel performance codes are either not available to AFC or have limitations and deficiencies in predicting AFC fuel performance. Advanced codes such as BISON are ready to be adapted and tailored to AFC analysis needs. The immediate need is to determine the temperature response of AFC fuels under various irradiation conditions and fuel-clad contact behavior and understand the species migration and redistribution, including lanthanide penetration into the clad. The prediction of the fuel- 
clad contact under symmetric and axisymmetric conditions is critical to determine fuel failure due to fuelcladding-chemical-interaction (FCCI) mechanisms. Table 1 shows the key models in various metal fuel performance codes and the desired capabilities in an analysis tool (BISON-METAL) built on the current MOOSE/BISON framework by incorporating state-of-the-art knowledge and constitutive models and properties of AFC metal fuels into the MOOSE/BISON framework. All other simulation requirements are determined and published. The short and long-term implementation plan is also developed and published in the AFC execution plan.

Table 1. Key Modeling in Metal Performance Analysis Tool

\begin{tabular}{|c|c|c|c|c|c|c|}
\hline Key Models & $\begin{array}{l}\text { LIFE-METAL } \\
\text { (Steady-State } \\
\text { and Design } \\
\text { Basis Accidents) }\end{array}$ & $\begin{array}{c}\text { SESAME } \\
\text { (Steady-State) }\end{array}$ & $\begin{array}{c}\text { ALFUS (Steady- } \\
\text { State) }\end{array}$ & $\begin{array}{c}\text { MACSIS } \\
\text { (Steady-State) }\end{array}$ & $\begin{array}{l}\text { Feast-metal } \\
\text { (Steady-State } \\
\text { and Transients) }\end{array}$ & $\begin{array}{l}\text { BISON-METAL } \\
\text { (Steady-State } \\
\text { and Transients) }\end{array}$ \\
\hline Developer & ANL & CRIEPI & CRIEPI & KAERI & MIT & INL-LANL \\
\hline $\begin{array}{c}\text { Fission Gas } \\
\text { Release and } \\
\text { Fuel Swelling }\end{array}$ & $\begin{array}{l}\text { Empirical } \\
\text { Correlation }\end{array}$ & $\begin{array}{l}\text { Empirical } \\
\text { Correlation }\end{array}$ & $\begin{array}{c}\text { Mechanistic } \\
\text { Model, based on } \\
\text { UO2 Fuel }\end{array}$ & $\begin{array}{l}\text { Mechanistic } \\
\text { Model, based on } \\
\text { UO2 Fuel }\end{array}$ & $\begin{array}{l}\text { Mechanistic } \\
\text { Model, based on } \\
\text { Metallic Fuel }\end{array}$ & $\begin{array}{c}\text { Mechanistic } \\
\text { Models and Rate } \\
\text { Theory }\end{array}$ \\
\hline $\begin{array}{l}\text { Species } \\
\text { Transport }\end{array}$ & $\begin{array}{l}\text { Empirical } \\
\text { Correlation }\end{array}$ & $\begin{array}{l}\text { Chemical } \\
\text { Equilibrium } \\
\text { Model }\end{array}$ & $\begin{array}{c}\text { Thermo-transport } \\
\text { Theory }\end{array}$ & $\begin{array}{c}\text { Thermo-transport } \\
\text { Theory }\end{array}$ & $\begin{array}{c}\text { Thermo-transport } \\
\text { Theory }\end{array}$ & $\begin{array}{c}\text { Thermo-transport } \\
\text { Theory for } \\
\text { Multiple } \\
\text { Elements }\end{array}$ \\
\hline $\begin{array}{l}\text { Temperature } \\
\text { Distribution }\end{array}$ & 1D Model & 1D Model & 1D Model & 1D Model & 1D Model & 3D Model \\
\hline $\begin{array}{l}\text { Mechanical } \\
\text { Analysis }\end{array}$ & 1D Model & 1D Model & 2D Model & 1D Model & 1D Model & 3D Model \\
\hline FCCI & $\begin{array}{l}\text { Empirical } \\
\text { Correlation }\end{array}$ & $\mathrm{N} / \mathrm{Z}$ & $\begin{array}{l}\text { Empirical } \\
\text { Correlation }\end{array}$ & N.A & $\begin{array}{l}\text { Diffusion Model } \\
\text { based on } \\
\text { Precipitation } \\
\text { Kinetics }\end{array}$ & $\begin{array}{l}\text { Diffusion Model } \\
\text { based on } \\
\text { Precipitation } \\
\text { Kinetics }\end{array}$ \\
\hline Creep Fracture & $\begin{array}{c}\text { Cumulative } \\
\text { Damage Fraction } \\
\text { Model }\end{array}$ & N/A & N/A & N/A & $\begin{array}{l}\text { (1) Cumulative } \\
\text { Damage Fraction } \\
\text { Model (2) } \\
\text { Constrained } \\
\text { Diffusional } \\
\text { Cavity Growth } \\
\text { Model }\end{array}$ & $\begin{array}{l}\text { (1) Cumulative } \\
\text { Damage Fraction } \\
\text { Model (2) } \\
\text { Constrained } \\
\text { Diffusional } \\
\text { Cavity Growth } \\
\text { Model }\end{array}$ \\
\hline
\end{tabular}

\subsubsection{Assessment of BISON Fuel Performance Code to Develop a Metallic Fuel Analysis Tool [BISON-METAL]}

As a part of the development of BISON-METAL fuel analysis tool we examine the utility of BISON tool in addressing and resolving technical issues associated with the transmutation fuel development. The BISON metallic rodlet model was developed with accurate geometrical dimensions of the components and the as-run linear heat generation rate to provide a more accurate centerline temperature results for the FCRD irradiation experiments. The tool is used to investigate effects of fuel slug eccentricity and axial heat flow. Currently we are adding solid mechanics to thermal model, which will permit investigation of thermal expansion, fuel swelling, and mechanical contact.

Four cases were investigated, including one axisymmetric case and three cases in which either fuel slug, cladding, or both are displaced radially resulting in asymmetric gaps between the components. If both the cladding and the slug are offset, the maximum cladding temperature is $711 \mathrm{~K}$ (Figure 1).

Evidently, the eccentricity results in formation of the hot and cold sides in the capsule assembly. Results further point to the significant temperature difference between the hot and cold cladding sides. The highest temperature difference amounts to $99 \mathrm{~K}$ and is observed in the all-offset case. 


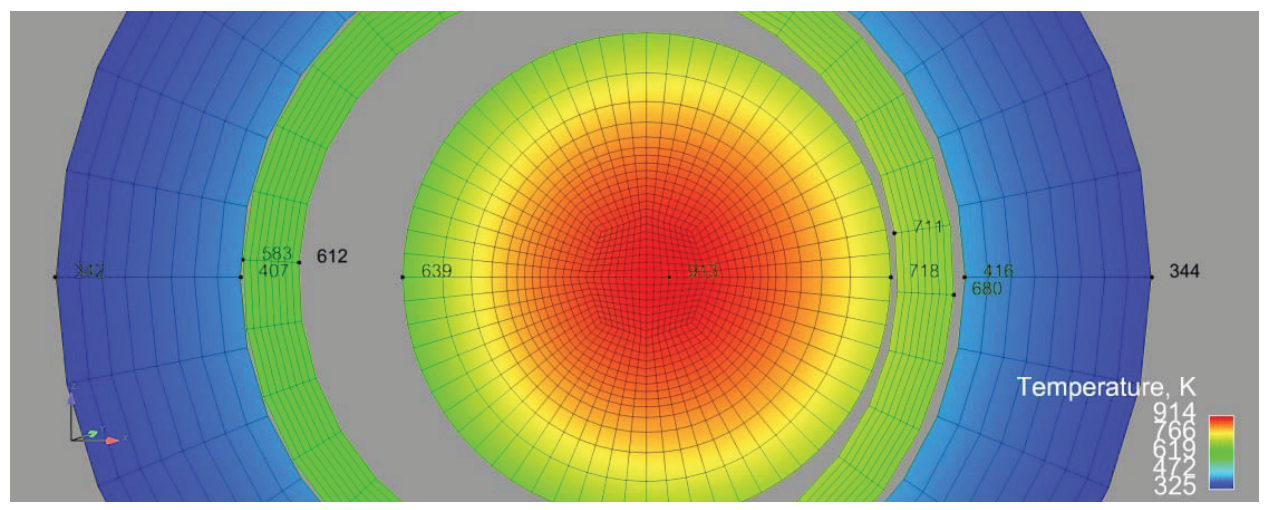

Figure 1. Temperature distribution for the all-offset case.

Figure 2 reveal that eccentricity results in an asymmetric stress loading in the fuel, cladding and the capsule. This is a direct consequence of the asymmetric temperature distribution. The most pronounced effect is seen in the all-offset case where the absolute value of stress in the hot side of the cladding is 3.24 times greater than the stress in the cold side of the cladding.

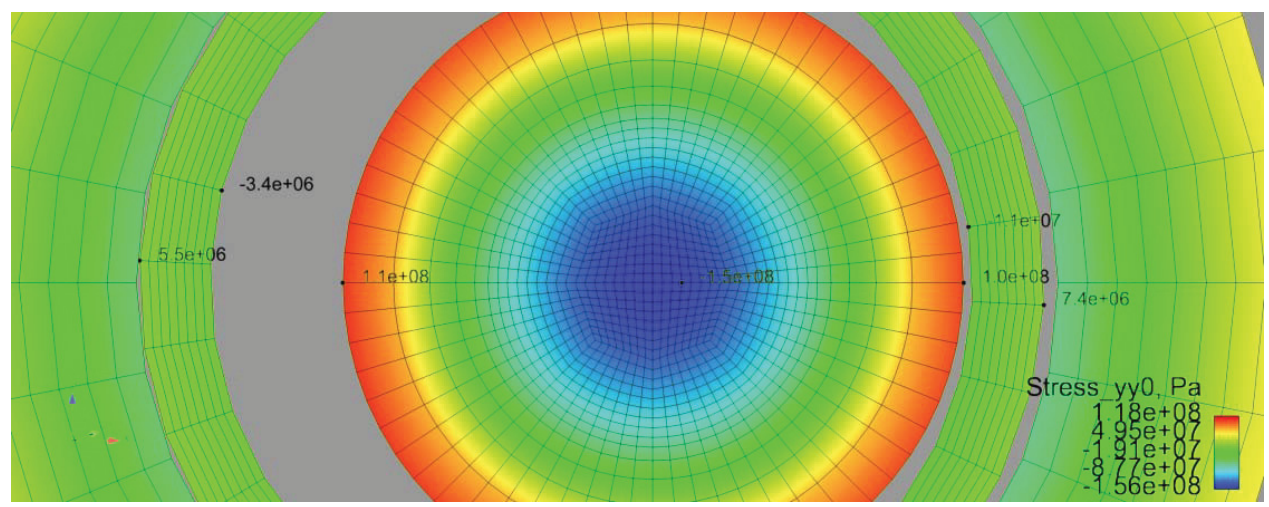

Figure 2. Distribution of axial stress for the all-offset case.

Expansion and bowing of the cladding is noted in the asymmetrical cases, with the maximum bowing observed in the all-offset case (Figure 3). Bowing of the cladding results in the increase of the claddingcapsule gap in the lower region of the hot side of the assembly. Increase of the gap leads to the temperature increase, which causes further increase of the thermal expansion of the hot side of the assembly, thus causing more cladding bowing. Therefore, in the asymmetrical cases there exists a positive feedback mechanism between the temperature asymmetry and the cladding bowing. 


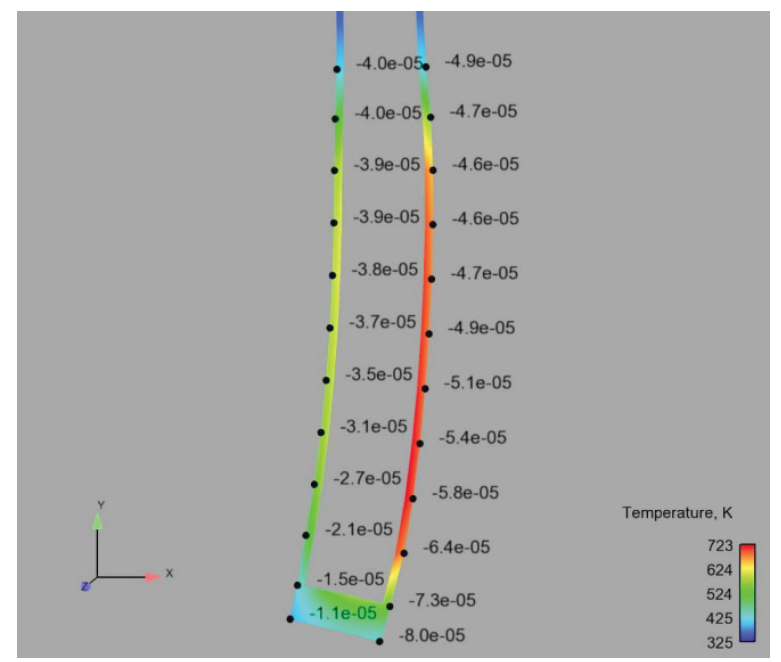

Figure 3. The shape of the cladding for the all-offset case. (Displacement is magnified by 100 times. The values of cladding-capsule radial gap are shown for each location marked with a black dot. The '-' sign signifies that the contact has not occurred, and the numeric value is equal to the gap in meters).

\subsubsection{Metal Fuel Validation Data}

A database for 10 Metallic Fuel Pins from experiment X425 irradiated at EBR-II, containing metallic fuel elements, was generated as a validation data for the BISON-METAL analysis tool. The database contains detailed operating conditions for 10 pins from this experiment at different stages of irradiation and corresponding experimental measurements of cladding strain. The pins considered are T401, T402, T405, T408, T413, T441, T442, T444, T446, and T473. The choice of the 10 pins is based on the fact that those pins have been used in all stages of irradiation of this experiment. Each pin data sheet contains data at 30 axial locations where first section at 0.02 is the bottom section of the pin. Data are generated at the end of each reconstitution of experiment. For example data are generated at the end of X425 after irradiation for 146.5 days. Temperatures and power densities listed at the end of each reconstitution are weighted average over all 8 or 7 runs within that period. Only measured data included here are the cladding strain proliforimetry data.

\subsection{Metal Fuel Performance Sensitivity Assessment}

Michael Todosow, todosowm@bnl.gov, BNL; Jess Gehin, ORNL; Abdellatif Yacout, ANL

BNL and Oak Ridge National Laboratory (ORNL) performed calculations to evaluate the performance and safety characteristics of a proposed concept by Logos Nuclear Systems based on the utilization of TRISO particle fuel in selected locations in a PWR assembly to burn the TRU from lightwater reactor (LWR) UNF. The TRISO particles would be in silicon carbide or graphite compact/stick that would be located in conventional Zircaloy clad; an example assembly configuration is shown in Figure 4. While the FY 2011 analyses focused on an initial assessment of this concept, the higher-level objective was to develop, qualify, and demonstrate a computational methodology for assessing the performance and safety characteristics associated with the use of innovative fuels in PWRs. These could include the FCM fuel currently being advocated by ORNL, and metallic fuel. 


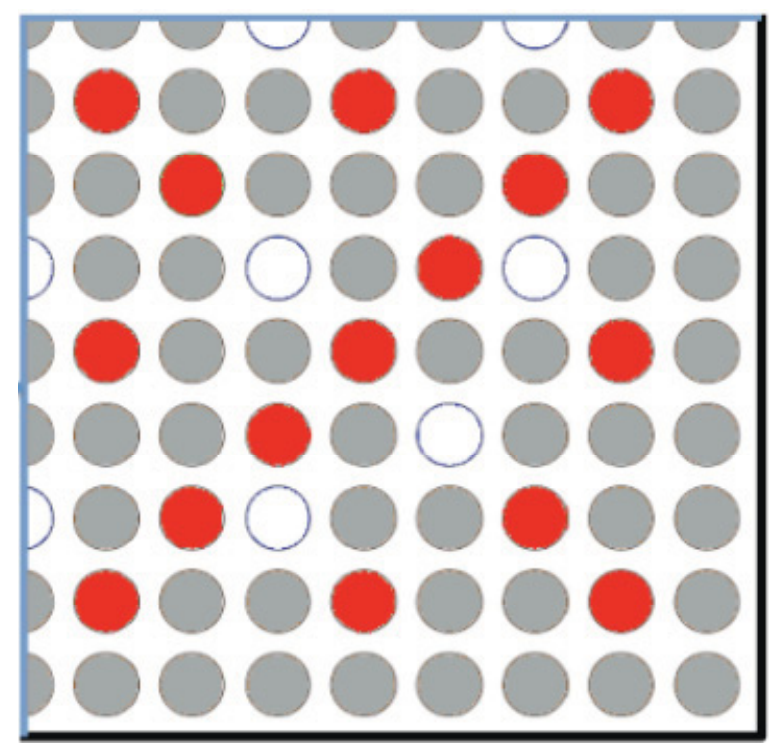

Figure 4. Quadrant of $17 \times 17$ PWR assembly with UOX rods (gray) and TRISO-TRU rods (red).

The study began with assembly calculations to compare to results presented by Logos, and determination of key reactivity coefficients (fuel and moderator temperature), and control worths (soluble boron and control rods), and a comparison to results for a conventional uranium oxide (UOX) fueled assembly. In order to aid in cross-section development for use in a core simulator, ORNL developed a simplified methodology for reflecting the double heterogeneity of the TRISO particle-in-stick geometry in the TRITON lattice assembly code that allowed automated branch calculations to reflect variations in fuel, moderator and control conditions that span the parameter space in the three-dimensional model

A three-dimensional core model for an AP1000 reactor at beginning of life (BOL) with standard UOX fueled assemblies was developed for the PARCS steady state and transient core simulator. Results for the BOL core power distribution and reactivity coefficients were compared to results in the Final Safety Analysis Report and showed reasonable agreement, as did the results for core power in a rod ejection accident (REA). A preliminary estimate of the performance of a fuel particle was obtained using an explicit LS-DYNA simulation (see Figure 5).

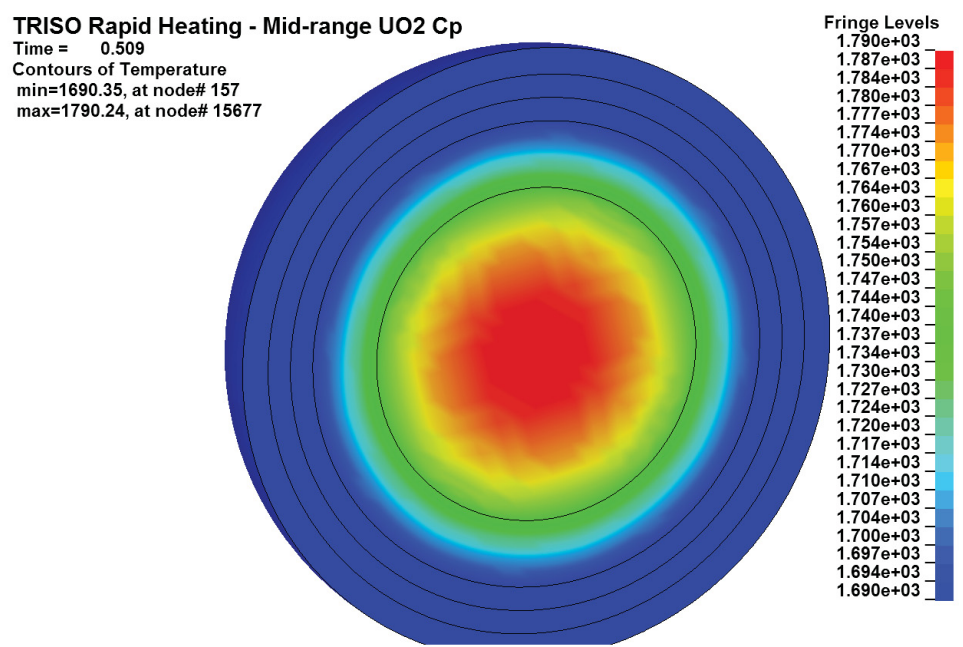

Figure 5. Temperature distribution within a TRISO particle at $0.509 \mathrm{sec}$. assuming a mid-range estimate for the specific heat. 


\subsection{Simulation of Casting of Metal Fuel Alloys}

J. Crapps, jcrapps@lanl.gov, D. Korzekwa, J. Galloway, N. Carlson, P. Sadasivan, C. Unal, LANL

The metal fuel casting project is working to develop a capability to simulate manufacturing processes for metallic fuel rods. The BSC at INL is being used as a representative process for this effort.

The requirements for metal casting processes can be broadly categorized into fluid flow capabilities, heat transfer capabilities and material science capabilities. The material science requirements are generally to model the solidification, species segregation and microstructure development. The details of the numerical coupling between these capabilities are also important in determining the physical phenomena that can be captured in the simulations.

Using TRUCHAS code, attempts were made to model filling with an argon atmosphere in the mold by assuming that the argon is incompressible. The first attempt assumed that the only vent for the argon is the 0.030 " diameter vent at the bottom of the rod mold. In this simulation the liquid metal plugged the vent and solidified early in time and the only way for the argon to leave the mold was by bubbling up through the crucible (Figure 6).
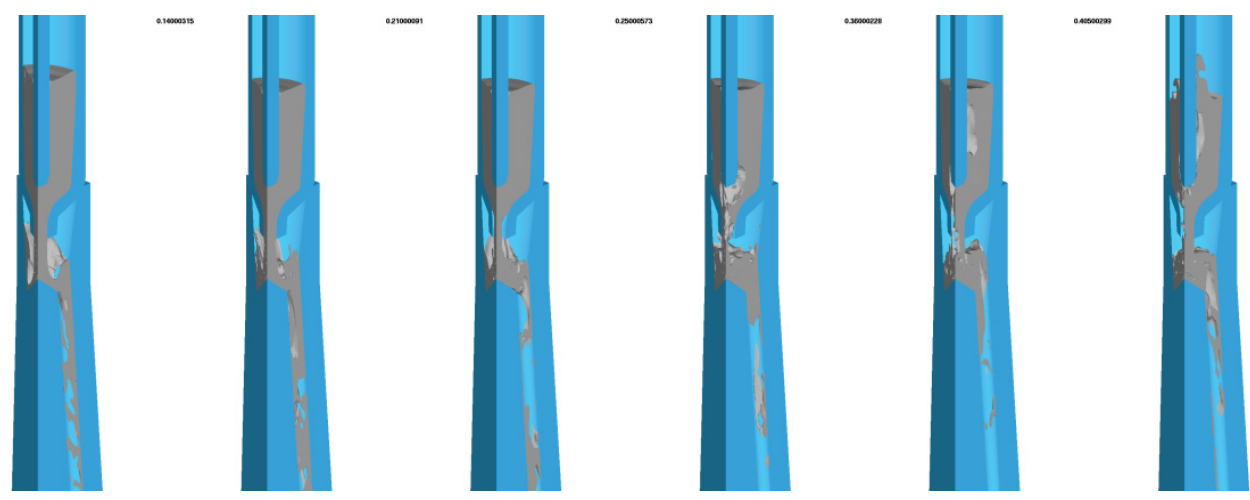

Figure 6. Snapshots from filling with vent only at the rod bottom

Since the surface tension model in Truchas cannot be used for the BSC simulations and Truchas cannot model the compressibility of the argon gas, an effort was made to evaluate these aspects of the flow behavior using FLOW3D. FLOW3D can model compressible flow, so it should be able to model the liquid as essentially incompressible and the argon as a compressible gas. FLOW3D also allows the specification of surface tension at the liquid-gas and liquid-mold interfaces. We evaluated the ability of FLOW3D to run reasonably resolved simulations of the BSC and assess the magnitude of the effects of surface tension and gas compressibility. The scalability of FLOW3D to large highly-resolved simulations is also investigated.

Figure 7 shows that while surface tension does not appear to have an appreciable effect on simulation results when filling an evacuated mold, it does affect the simulation results when the adiabatic bubble model is used to simulate a mold initially occupied with compressible argon. This seems reasonable in that the surface tension will resist the formation of bubbles that must create new surface area as they form at the liquid surface and move through the liquid. Figure 7 considers the four venting scenarios. In Figure 7, the left image for each scenario represents the final state of the simulation without surface tension while the right image represents the final state of the simulation including surface tension. Inclusion of surface tension in the model causes argon to become potentially trapped between different sections of the casting, a very undesirable situation. The best way to remedy this problem is to vent the mold at multiple locations; perhaps at the bottom, midway up, and at the base of the crucible. 
These results will be validated with Separate Effects Tests and Integral Effects Tests later in FY 2012. Nevertheless, for comparison Figure 8 shows the result of one of the BSC casting runs with argon in the mold. Two rod cavities produced pieces about 8 inches long, and it appears that one of those reached the vent in the bottom of the mold and the other did not. The third rod cavity produced two shorter pieces. The results are qualitatively similar to the FLOW3D results with surface tension and the adiabatic bubble model. We concluded that FLOW3D is the tool we need to use the address the optimization of casting conditions in FY 2012. THRUCHAS code will be used for simulation of Am volatilization and multispecies diffusion tests conducted by INL.

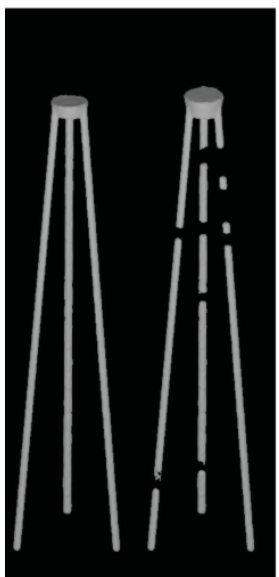

Bottom

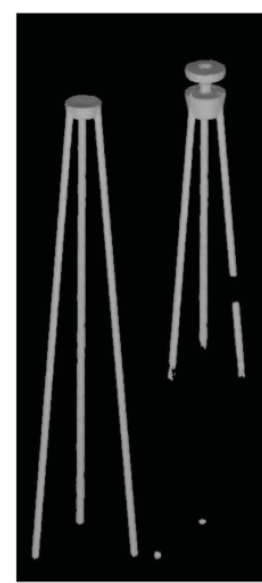

Midway

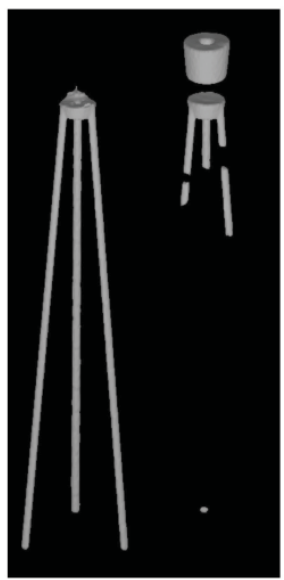

Top

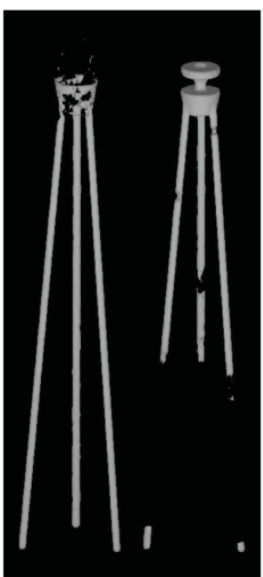

Crucible

Figure 7. Final Simulation state for four venting scenarios comparing model with and without surface tension. The model without surface tension is represented by the left image in each case while the model including surface tension is represented by the right image. Including surface tension when the mold is initially occupied by argon makes significant differences in the simulation results. Appropriate venting at multiple locations in the mold will help to remedy the problem of argon becoming trapped between different sections of the U-10wt\% Zr.

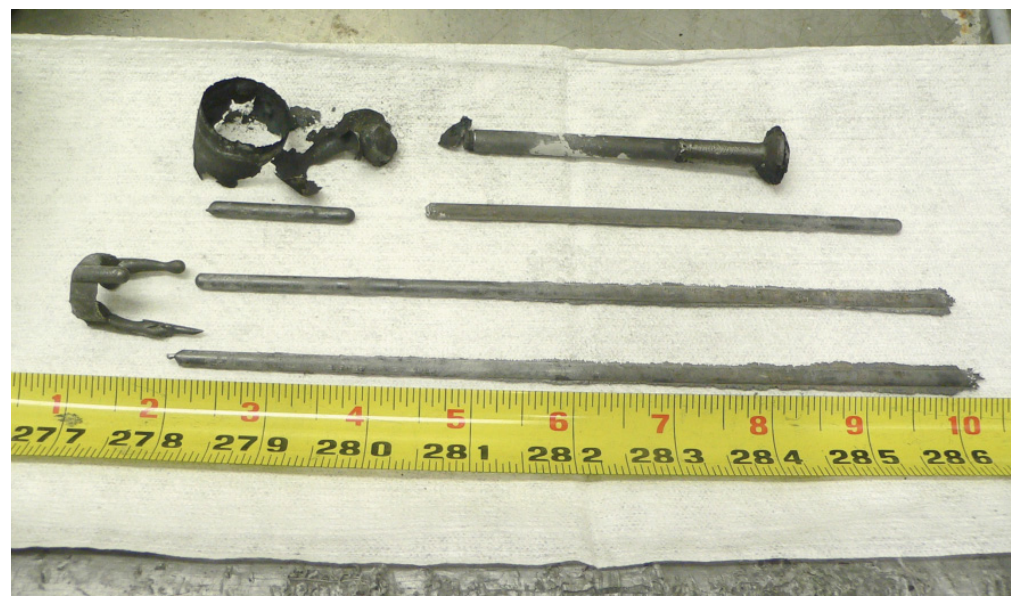

Figure 8. Result of BSC casting with argon initially in the mold. Each rod cavity is 10 inches long. Photo courtesy of Randall Fielding, INL. 
The following specific conclusions were drawn:

- Inclusion of a compressible second fluid using FLOW3D's adiabatic bubble model significantly affects fill time when compared to a simulation considering an evacuated mold. This suggests a modeling the effects of the argon should be included in simulations.

- The best single position to vent the argon was at the base of the crucible. However, a venting scheme using multiple vents will probably yield the best results.

- When considering a simulation including compressible argon and surface tension, pockets of argon can become isolated causing a break in the cast rod. This is an unacceptable condition and suggests that surface tension is an important parameter to include in the model.

- A coarse version of a FLOW3D simulation of the BSC scaled well up to 8 cores using Intel Xeon 5570 and Xeon X7560 processors using the shared memory parallel version.

- A reasonably fine FLOW3D simulation of the BSC produced results using surface tension and the adiabatic bubble approximation in 120 hours of simulation time on 8 cores. This is acceptable performance for resolving the necessary detail in the BSC, and indicates that FLOW3D is a reasonable candidate for further development in this program.

- The distributed memory version of FLOW3D should be evaluated, since it will probably give substantially better performance and scaling on systems or clusters of more than 8 processors.

\title{
3.4 Sintering Simulation of Oxide Fuels
}

\author{
E. Luther, eluther@lanl.gov, M. Bogdan, C. Unal, LANL
} V. Tikare, SNL

Oxide fuel pellets are cold pressed and sintered from a powder feedstock. A better understanding of the properties of the starting materials, improving the control of the processes that are used to fabricate fuel pellets and understanding how the properties of these pellets change in service, represent important aspects that are being studied via experiments, models and simulations.

The sintering behavior of a ceramic is best characterized by dilatometry. This technique measures the change in length of a material as it is heated under a controlled atmosphere. By measuring the sintering behavior of material pressed under identical conditions sintered over a range of CHRs, a family of sintering curves can be generated. These curves can be collapsed into an MSC and used to predict the sintering behavior of the material for any arbitrary sintering profile.

The uranium oxide powder used in this study was purchased from AREVA and is produced by a "dry conversion process" of UF6 to UO2. Being a reactor grade material, this powder has extremely low impurity levels. The average particle size is 2.8 microns and the average surface area is $2.3 \mathrm{~m} 2 / \mathrm{g}$. (Figure 9) is a scanning electron microscopy (SEM) image showing the general size and morphology of the powder. 


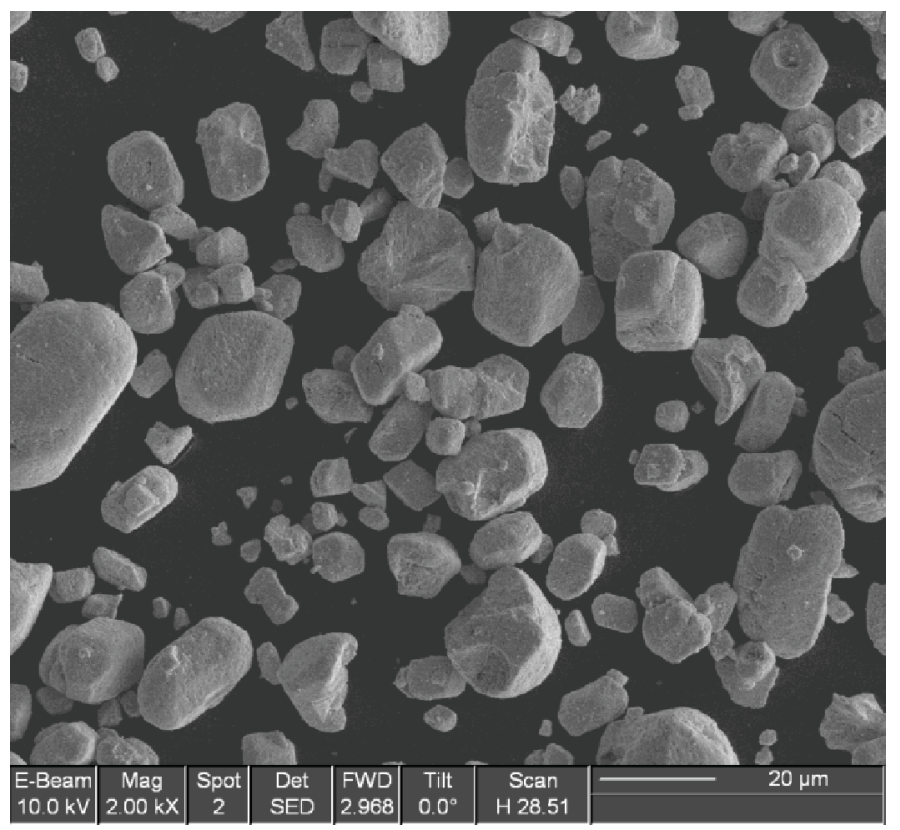

Figure 9. SEM image of as-received depleted uranium oxide from AREVA.

As received, the $\mathrm{O} / \mathrm{M}$ ratio for the AREVA powder was found to be $\mathrm{O} / \mathrm{M}=2.16$. The sintering profiles were determined at CHRs of 5, 10, 15 and $20^{\circ} \mathrm{C} / \mathrm{min}$.

The powder received from AREVA was reduced in order to achieve an $\mathrm{O} / \mathrm{M}=2.1$ and $\mathrm{O} / \mathrm{M}=2.0$ compositions. Then, experiments were performed to obtain the sintering profiles for CHRs of 5, 10, and $20^{\circ} \mathrm{C} / \mathrm{min}$ for $\mathrm{O} / \mathrm{M}=2.1$ case. For $\mathrm{O} / \mathrm{M}=2.0$, we determined the sintering profiles for CHRs of 10 and 20 ${ }^{\circ} \mathrm{C} / \mathrm{min}$.

Apparent activation energies for the sintering process in the AREVA powder were obtained as 359 $\mathrm{kJ} / \mathrm{mol}, 383 \mathrm{~kJ} / \mathrm{mol}$, and $203 \mathrm{~kJ} / \mathrm{mol}$ for $\mathrm{O} / \mathrm{M}=2.16, \mathrm{O} / \mathrm{M}=2.10$ and $\mathrm{O} / \mathrm{M}=2.0$, respectively.

The $359 \mathrm{~kJ} / \mathrm{mol}$ activation energy we obtained for $\mathrm{O} / \mathrm{M}=2.16$ can be compared with the $299 \mathrm{~kJ} / \mathrm{mol}$ activation value obtained by Dehaudt et al. (Ph. Dehaudt, L. Bourgeois, H. Chevrel, "Activation Energy of UO2 and UO2+x on Sintering," J. Nuc. Matls., 299, 250 (2001)) for O/M=2.17. Their analysis of the CHR sintering data employed the related Dorn method that also relies on the assumption that the sintering process is dominated by a single densification mechanism, similar to the MSC theory described above. However, Dehaudt et al. biased their derivation of the activation energy towards slow CHR by carrying out experiments for $1.25,3,5$ and $8.3^{\circ} \mathrm{C} / \mathrm{min}$.

For all compositions, we find that the scatter in the fit is minimal indicating excellent reproducibility of the data. This indicates that independent of the $\mathrm{O} / \mathrm{M}$ value used for the sintering process, the sintering process is dominated by only one diffusion mechanism and the MSC theory is applicable to describing the sintering of oxide nuclear fuel pellets (See Figure 10). 


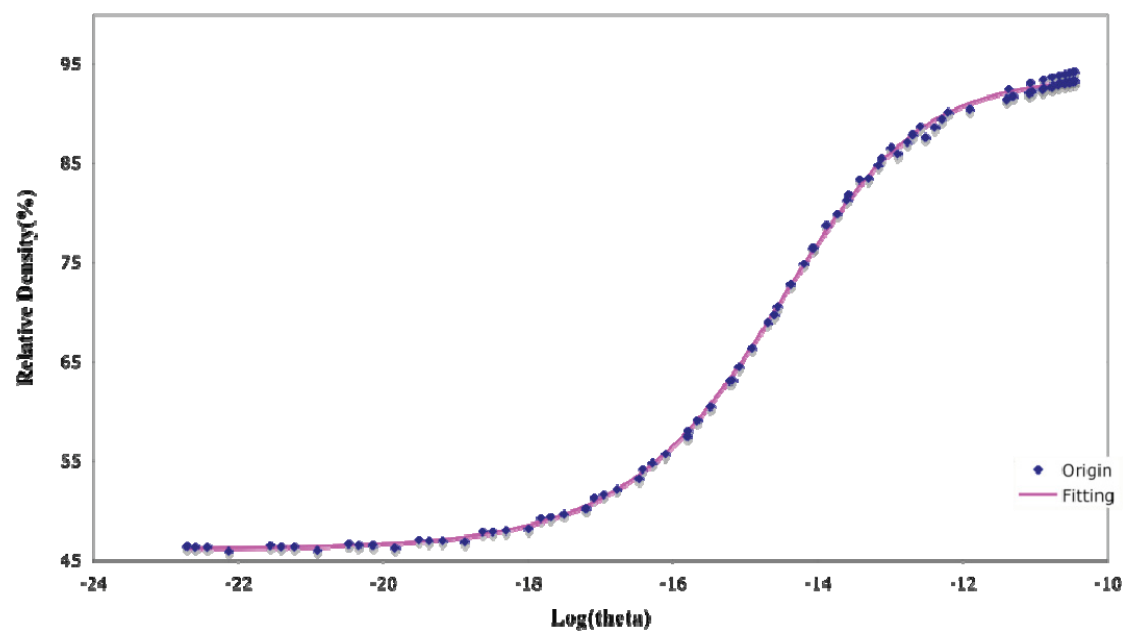

Figure 10. Master Sintering Curves for AREVA powder at different O/M ratio of 0.16 . 


\section{METALIL-BASED FUELS TECHNOLOGIES}

Technical Lead: J. R. Kennedy, rory.kennedy@inl.gov, INL

R. Fielding, M. Fig, J. Giglio, I. Glagolenko, D. Hurley, T. Hyde, D. Janney, D. Kotter, P. Lessing, R. Mariani, T. O'Holleran, M. Okuniewski, J. Riesterer, B. Sencer, J. Smith, L. Squires, and K, Wright, INL; A. E. Wright, T. K. Kim, D. Yun, A. M. Yacout, Y. S. Kim, W. D. Pointer,

A. R. Kraus, T. H. Bauer, and K. Grimm, ANL;

R. Omberg, A. L. Doherty, K. J. Geelhood, C. H. Henager,

C. A. Lavender, D. L. Love, and C. L. Painter, PNNL

The FY 2011 accomplishments from the Metallic Fuel Technologies Technical Area produced significant achievements in all work areas despite a prolonged radiological work stand down. The technical area completed eight Level 2 and 19 Level 3 milestones in FY 2011. Some of the highlight accomplishments for FY 2011 include: the radial profiling of thermal diffusivity at $50 \mu \mathrm{m}$ spatial resolution (highest known spatial resolution capability) on four irradiated metal fuel samples using the newly implemented Scanning Thermal Diffusivity Microscope (STDM); the installation and application of radioactive materials into the Electron Probe MicroAnalyzer (EPMA); the continuing development of the Focused Ion Beam (FIB) and Micro-focus X-ray Diffractometer ( $\mu$-XRD) to higher levels of radioactive materials; the fabrication of 60 transmission electron microscopy (TEM) samples for irradiation in the High-Flux Isotope Reactor (HFIR) rabbit system; the development of a technique for the fabrication of annular fuel forms; the fabrication and characterization of the AFC-3A and AFC-3B irradiation test fuels and experiments; the development and testing of a method for trace element analysis of Fe and $\mathrm{Si}$ on fuels; the completion of the fabrication of the glove-box advanced casting system (GACS); the initiation of the construction of a multifunctional prototype instrument for laser ultrasound measurements; the testing of a radio frequency/surface acoustic wave wireless transmission system for in-pile measurement applications; the initial development of a thermo-acoustic sensor for in-pile measurements; test runs of the $\mathrm{AmO}_{2}$ reduction/distillation apparatus with surrogates; the successful development and realization of a method to produce representative porous metal materials for investigations of fission product migration mechanisms; significant advancement in the fundamental studies on constituent migration and FCCI in metal fuels. Highlights according to work area will be presented below.

\section{Milestones Gompleted}

Level 2

- Draft report documenting FCCI studies. (12/15/2010)

- Produce Draft Report on Fundamental Fuel Constituent Migration. (3/31/2011)

- Initiate construction of a multifunctional prototype instrument for laser ultrasound measurements. $(6 / 27 / 2011)$

- Produce status report on LWR Metallic Fuel Concept. (8/12/2011)

- Produce status report on Advanced Metallic Fuel Concept for Reliable Performance to Ultra-high Burnup together with Argonne National Laboratory (ANL). (8/29/2011)

- Produce status report on AFC-3 irradiation experiment fabrication. (9/29/2011)

- Produce draft report on micro-scale laser based thermal diffusivity measurements on nuclear fuels. $(9 / 29 / 2011)$

- Produce status report on Advanced Metallic Fuel Concept for Reliable Performance to Ultra-high Burnup together with INL. (9/30/2011) 


\section{Level 3}

- Submit a status report on effusivity, melting point, and multifunction technique development. $(10 / 12 / 2010)$

- Issue Advanced Metallic Fuel Concept Status Report. (11/29/2010)

- Design \& Analysis Dispersion Fuel Research and Development Plan. (12/12/2010)

- General Report on Fuel Characterization. (12/15/2010)

- Submit a status report on fundamental diffusion properties and techniques. $(12 / 15 / 2010)$

- Installation of the Shutdown Cooling System (SCS) control system on the Advanced Fuel Cycle Initiative (AFCI) sintering furnace. (12/15/2010)

- End of year report on metallic fuel sample preparation activities. (12/20/2010)

- Initiate characterization of AFC-3 fuel materials. (2/16/2011)

- Conduct initial research on the development of an annular fuel fabrication process for $1.5 \mathrm{in}$. long slugs. $(3 / 4 / 2011)$

- Complete Fundamental FCCI Studies Update Presentation for Quarterly Meeting. (3/23/2011)

- Report on current state of the art for analytical chemistry support for fuels characterization. $(3 / 31 / 2011)$

- Produce Irradiation Test Fabrication Report on HFIR Rabbit Test Samples. (4/29/2011)

- Complete Fundamental Thermophysical Properties Update Presentation for Quarterly Meeting. $(6 / 28 / 2011)$

- Complete Fundamental Fission Products Behavior Update Presentation for Quarterly Meeting. $(6 / 28 / 2011)$

- Complete fabrication of AFC-3 fuel. (6/28/2010)

- Complete AFC-3 experiment ready for ATR insertion. (9/7/2011)

- Complete fabrication of at least 12 characterization castings. (9/29/2011)

- Complete Fundamental Element Redistribution Studies Update Presentation for Quarterly Meeting. (9/30/2011)

- Produce draft status report on instrumentation development. (9/30/2011)

\section{Collaborations}

- University of Idaho

- University of Nevada, Las Vegas

- Utah State University

- Colorado School of Mines

- Texas A\&M University (TAMU)

- University of Central Florida

- University of Missouri 
- Georgia Institute of Technology

- Korea Atomic Energy Research Institute (KAERI)

- Institute for Transuranium Elements (ITU)

\section{Publications}

B. Beeler, B. Good, S. Rashkeev, C. Deo, M. Baskes, and M. Okuniewski, First Principles Calculations for Defects in U, Journal of Physics Condensed Matter, vol. 22, n. 50 (2010), 505703.

D. H. Hurley, M. Khafizov, S. Shinde, Hurley, D. Measurement of Kaptiza Resistance Across a Bicrystal Interface, Journal of Applied Physics, 109, 83504 (2011).

D. E. Janney, J. R. Kennedy, “As-cast Microstructures in U-Pu-Zr Alloy Fuel Pins with 5-8wt\% Minor Actinides and 0-1.5wt\% rare-Earth Elements," Materials Characterization, (2011).

Michael Myers, Bulent H. Sencer, Lin Shao, "A multi-scale model of localized heating caused by ion bombardment", Nuclear Instruments and Methods, (2011).

R. R. Mohanty, J. Bush, M. A. Okuniewski, Y. H. Sohn, Thermotransport in $\gamma($ bcc) U-Zr alloys: A phase-field model study, Journal of Nuclear Materials, vol. 414 (2011), 211.

J. I. Cole, T. P. O'Holleran, D. D. Keiser Jr., and J. R. Kennedy," Out-of-pile Effects of Lanthanides on Fuel-Cladding Compatibility", Journal of Nuclear Materials, (2011).

Xing, C., Z. Hua, H. Ban, D. Hurley, and J. R. Kennedy, Evaluation of Uncertainties of One-Directional Analytical Model for Thermoreflectance Technique, ASME/JSME 2011 8th Thermal Engineering Joint Conference, Honolulu, HI, AJTEC2011-44539.

Xing, C., C. Jensen, H. Ban, R. Mariani, and J. R. Kennedy, An Electromotive Force Measurement System for Alloy Fuels, ASME 2010 International Mechanical Engineering Congress and Exposition, Vancouver, British Columbia, Canada, IMECE2010-39457.

Lanthanides in Metallic Nuclear Fuels: Their Behavior and Methods for Their Control,” Robert D. Mariani, Douglas L. Porter, Thomas P. O’Holleran, Steven L. Hayes, J. Rory Kennedy, Journal of Nuclear Materials, in press.

B. Beeler, B. Good, S. Rashkeev, C. Deo, M. Baskes, and M. Okuniewski, First Principles Calculations of the Stability and Incorporation of Helium, Xenon, and Krypton in Uranium, Journal of Nuclear Materials, accepted, in press.

Changhu Xing, Colby Jensen, Heng Ban, Robert Mariani, and J. R. Kennedy, Electromotive Force Measurement System for Material structure Investigation, Journal of Thermal Sciences and Engineering Applications (submitted).

\section{Patent Applications}

R. D. Mariani, "Zirconium-based Alloys, Nuclear Fuel Rods and Nuclear Reactors Including Such Alloys and Related Methods," U.S. Patent Application No. 13/021,480, filed February 4, 2011.

\subsection{Fuel Feedstock Preparation}

P. Lessing, paul.lessing@inl.gov, INL

There is a continuing need to document and secure feedstock materials within the FCRD program for current and future use. This need also requires establishment of methods and techniques to produce feedstock materials at desired purity levels. During FY 2011, the objectives for Fuel Feedstock were to: (1) reduce $\mathrm{NpO}_{2}$ to $\mathrm{Np}$ metal using molten salt reduction and then purify the resulting Np metal; (2) build 
a small size, high temperature distillation/condensation device to extract Am from $\mathrm{Am}-\mathrm{Pu}$ and Np-Am alloy feedstocks.

The molten salt reduction method for securing $\mathrm{Np}$ metal from $\mathrm{NpO}_{2}$ feedstocks was demonstrated in FY 2010 and is illustrated in Figure 11. This process produces Np metal of approximately 97\% purity. Work was planned and approvals secured for experiments designed to purify the $97 \% \mathrm{~Np}$ material. Melting in $\mathrm{MgO}$ crucibles under vacuum and also hydriding/de-hydriding experiments were approved using a tube furnace located in an inert atmosphere Pu qualified glove-box.

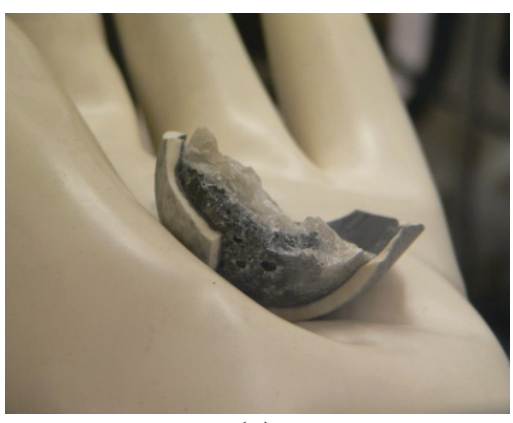

(a)

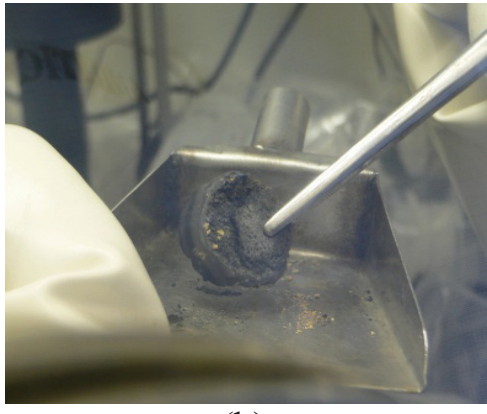

(b)

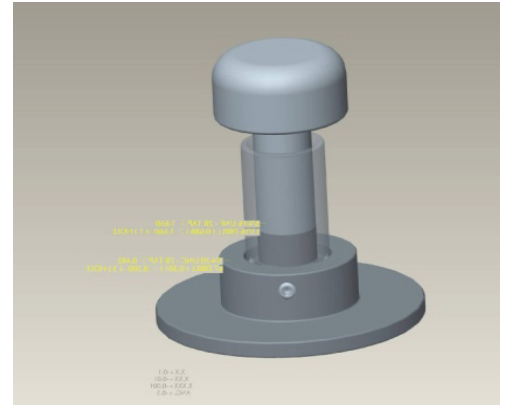

(c)

Figure 11. (a) Np metal and molten salt in bottom of MgO crucible, (b) removed, (c) hand mill for crushing to remove Np "nugget" for analysis or further purification.

The process to extract "pure" Am from 3\% Np-Am or 20\% Am-Pu alloys is outlined in Figure 12. The furnace was acquired and the tantalum distillation column (including the "cold" finger) was designed and fabricated. In addition, a special Am detector was designed, fabricated, and tested using various gamma sources. This detector sits outside the furnace and can measure the concentration of $\mathrm{Am}$ or $\mathrm{Pu}$ at various locations within the furnace. The detector will be used to indicate the optimum furnace temperature and optimum "cold finger" position that will give the maximum deposition rate of Am. Details of the furnace design are shown in Figure 13. The tube and condensation hardware (olive green color) are constructed from Ta metal. Most of the "cold finger" is constructed from high purity copper (gold color) while the heat shields and tip are constructed from Ta metal (olive green color). 


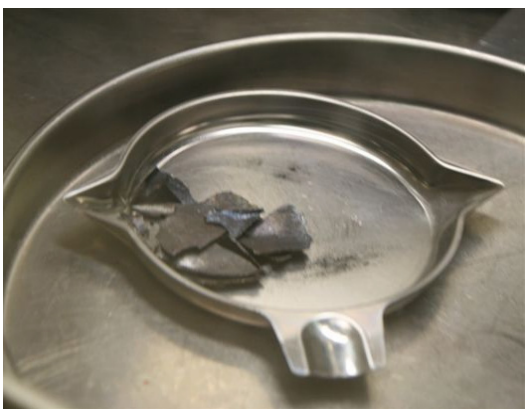

(a)

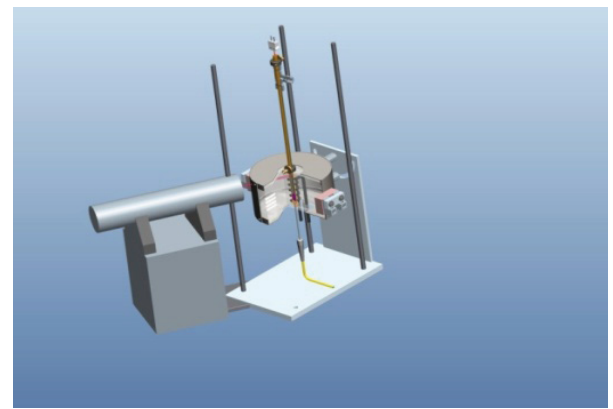

(d)

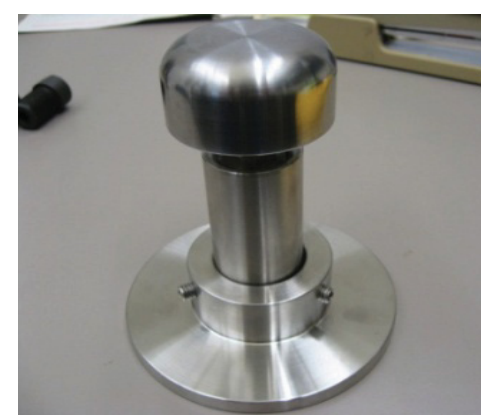

(b)

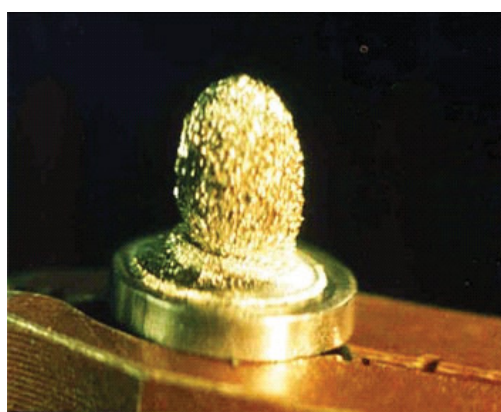

(c)

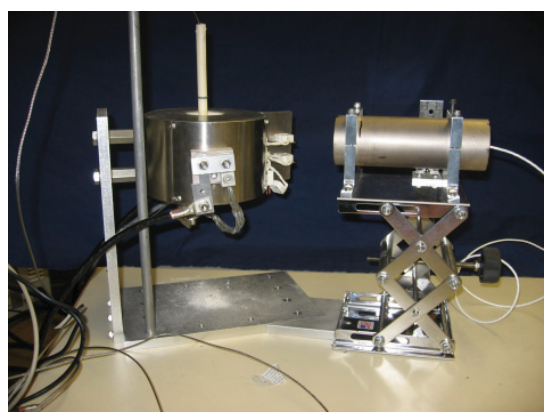

(e)

Figure 12. (a) Am-Np feedstock, (b) hand mill crushing, c) condensed Am (ITU), (d) drawing of small scale Am distillation apparatus with gamma detector, and (e) actual gamma detector (head).

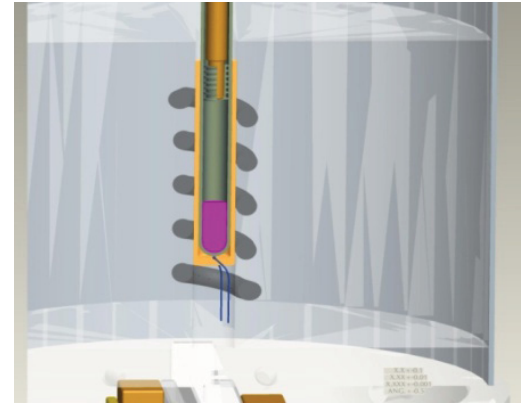

(a)

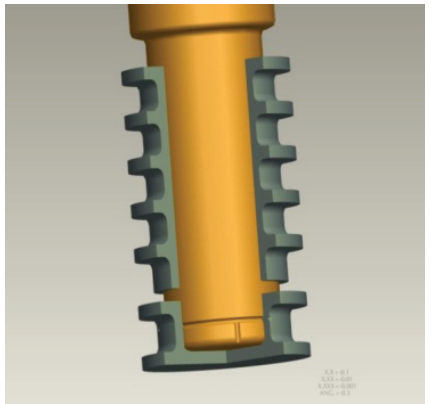

(b)

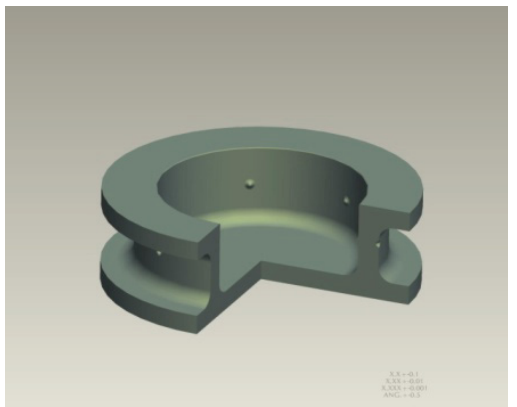

(c)

Figure 13. Details of the Am distillation/condensation apparatus: (a) heating element, tantalum tube, and molten metal, (b) Cu cold finger (gold color) with heat shield and cap, and (c) Ta deposition cap (removable).

Test runs of the apparatus were conducted in an air atmosphere using stainless steel tubing and Mn as a surrogate for Am. These experiments worked well with a high deposition rate of $\mathrm{Mn}$ at temperatures as low as $1000^{\circ} \mathrm{C}$ (using ceramic rather than Ta tube). A resulting Mn metal deposit is shown in Figure 14. 


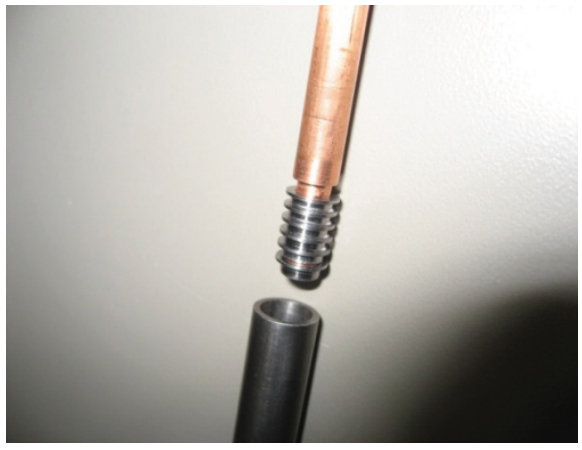

(a)

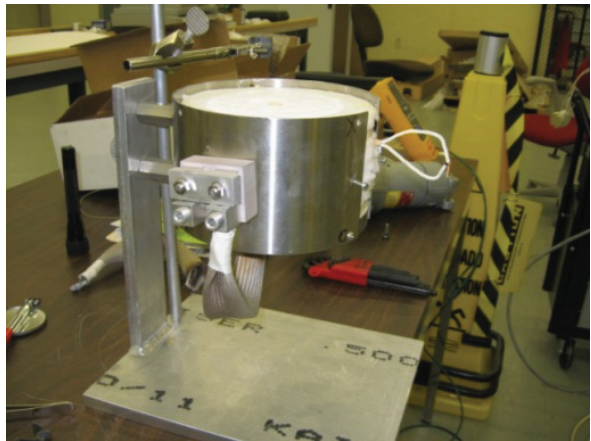

(c)

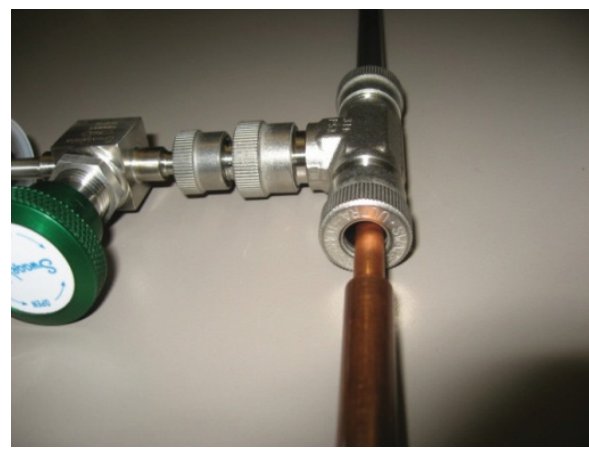

(b)

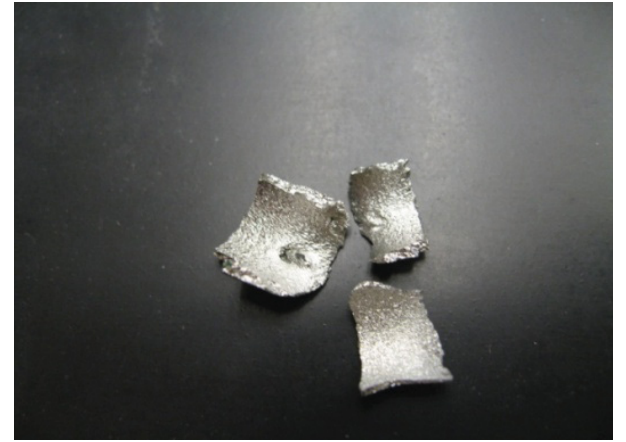

(d)

Figure 14. (a) Cold finger with Ta tip being inserted into Ta distillation tube, (b) outer end of long copper cold finger, (c) high temperature $\left(1600^{\circ} \mathrm{C}\right)$ furnace, and (d) Mn metal obtained from a test run.

Quality assurance (QA) approved software was written that will allow control of the furnace temperature and simultaneously provide an interpretation of the gamma ray spectrum. Operating procedures and related process work sheets were also written for operation of the systems during conduct of the evaporation/condensation experiments.

\subsection{Enhanced Analytical Techniques}

\section{J. Giglio, jeffrey.giglio@inl.gov, INL}

The FY 2011 objective was to review the state-of-the-art in analytical chemistry for fuels characterization and to develop an analytical method for the determination of $\mathrm{Fe}$ and $\mathrm{Si}$ in fuel samples. The analytical chemistry method development for determination of Fe and Si progressed in the area of Inductively Coupled Plasma-Atomic Emission Spectroscopy (ICP-AES). The method for the determination of Fe and $\mathrm{Si}$ via ICP-AES yielded quantitation limits (15 sigma times dilution factors) for $\mathrm{Si}$ of $25 \mu \mathrm{g} / \mathrm{g}$ and for Fe of $10 \mu \mathrm{g} / \mathrm{g}$. The primary AES method advance was in the development of a new dissolution strategy and applying the method to $\mathrm{U}$ bearing samples. In the traditional strategy of $\mathrm{Si}$ dissolution, the sample was subjected to a nitric acid/hydrofluoric (HF) acid mixture over a period of weeks with occasional agitation. The resulting dissolution resulted in large amounts of precipitated $U$ and un-dissolved sample on the bottom of the container. The new dissolution technique utilizes a hydrochloric $\mathrm{acid} / \mathrm{HF}$ acid mixture. This combination results in complete dissolution with no un-dissolved solid and a clear solution. The solution was analyzed via ICP-AES. The results were positive in that the method Quality Control (spikes, duplicates, and blanks) resulted in excellent analytical figures of merit. The ICP-AES method for Si determination is faster, more accurate, more precise, and less prone to interferences than the traditional dissolution method with spectroscopic detection. To further improve detection levels, an Inductively Coupled Plasma-Mass Spectrometry (ICP-MS) method is being 
developed that will utilize a dynamic reaction cell (DRC). The DRC will reduce the isobaric interferences on $\mathrm{Fe}$ (i.e., $\mathrm{ArO}^{+}$), thus allowing for lower detection limits.

\title{
4.3 Fabrication Technique Development
}

\author{
R. Fielding, randall.fielding@inl.gov, INL
}

The advanced fuel fabrication work package is tasked with developing fabrication methods for metallic fuel that meet the grand challenge of less than $0.1 \%$ fuel loss during fabrication and supporting fuel irradiation test specimen fabrication through fabrication development and parameter optimization. This year's focus was support of the HFIR rabbit irradiation tests, support of the AFC-3A and -3B irradiation tests, and development and installation in an inert atmosphere Pu qualified glovebox.

\subsubsection{HFIR Rabbit Sample Fabrication Support}

HFIR rabbit test support included developing parameters to cast uranium, uranium-10wt $\%$ zirconium (U-10Zr), uranium-15wt\% zirconium (U-15Zr), and uranium-20wt\% zirconium (U-20Zr) alloys. Rabbit tests were configured to accept several TEM samples. These samples were essentially discs, $3 \mathrm{~mm}$ in diameter by approximately $150 \mu \mathrm{m}$ in thickness. The difficulty in directly casting such specimens required the casting of rods ( $\sim 12 \mathrm{~mm}$ in diameter by $100-150 \mathrm{~mm}$ in length) as seen in Figure 15 . Discs were cut from the rods, which were thinned and several $3 \mathrm{~mm}$ diameter discs punched from these. The 3 $\mathrm{mm}$ discs were finally thinned to the appropriate thickness after punching. The larger discs were cut using a water cooled abrasive saw set at a low feed rate and approximately 500 RPM.

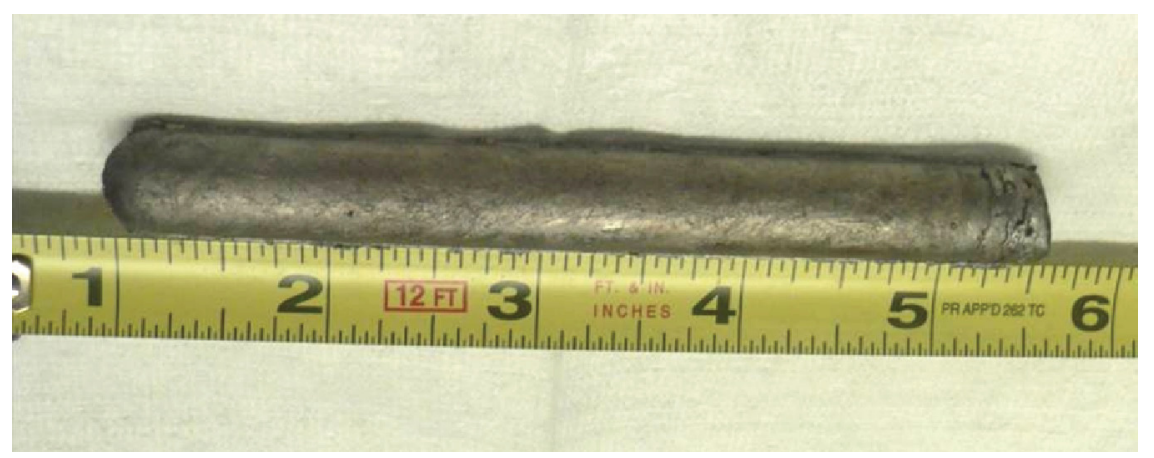

Figure 15. Typical example of cast product for TEM disc preparation (scale is in inches).

\subsubsection{Annular Fuel Casting Development}

The AFC-3A and AFC-3B irradiation test fuels included several annular fuel forms. Except for some limited experience with annular fuel forms in the Dounreay Fast Reactor, annular metallic fuel pins have not been generally produced. A lab scale casting process was the fabrication route of choice with other metal forming/cutting techniques such as extrusion, forging, or drilling (used alone or in conjunction with casting) considered as secondary options. Six different casting methods were initially attempted: (1) gravity arc casting with a mostly closed mold, (2) lab scale injection arc casting, (3) gravity casting using a Vacuum Pressure Casting Machine, (4) centrifugal casting, (5) gravity arc casting with open mold, and (6) counter gravity injection casting (CGIC). The casting development process and results are described in the following.

\section{Gravity Arc Gasting}

The majority of the AFC-1 and AFC-2 series of irradiation test specimens were gravity cast using arc casting techniques. For the annular fuel pins, the mold included graphite plug stabilized glass or graphite core. A typical mold is shown in Figure 16. Initial results showed that although the material would flow into the mold, it would not flow around the core consistently and would often flow only a short distance. 
Several modifications were attempted, including reduced pressure and increased zirconia lubricant, but did not increase the quality of the product. Figure 17 shows examples of typical specimens.

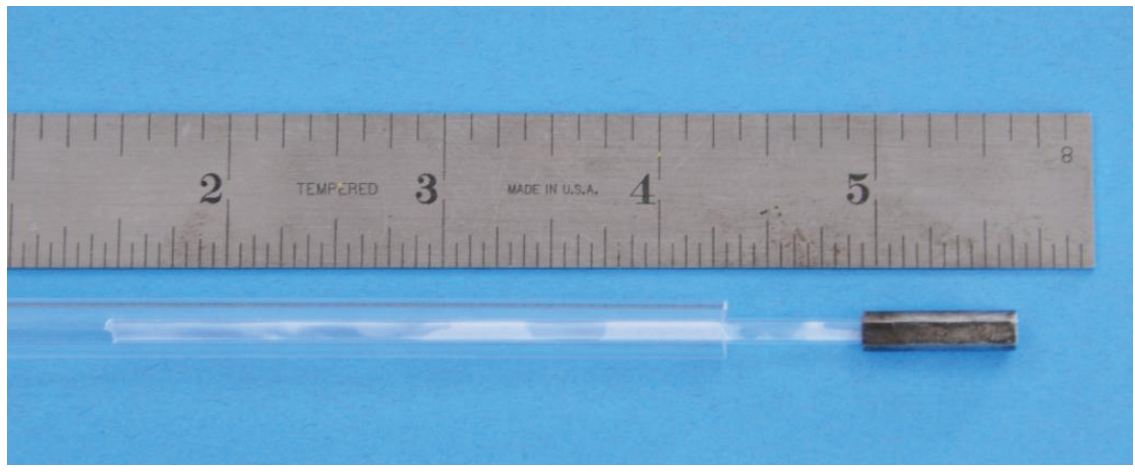

Figure 16. Quartz mold used for arc casting of annular pins.

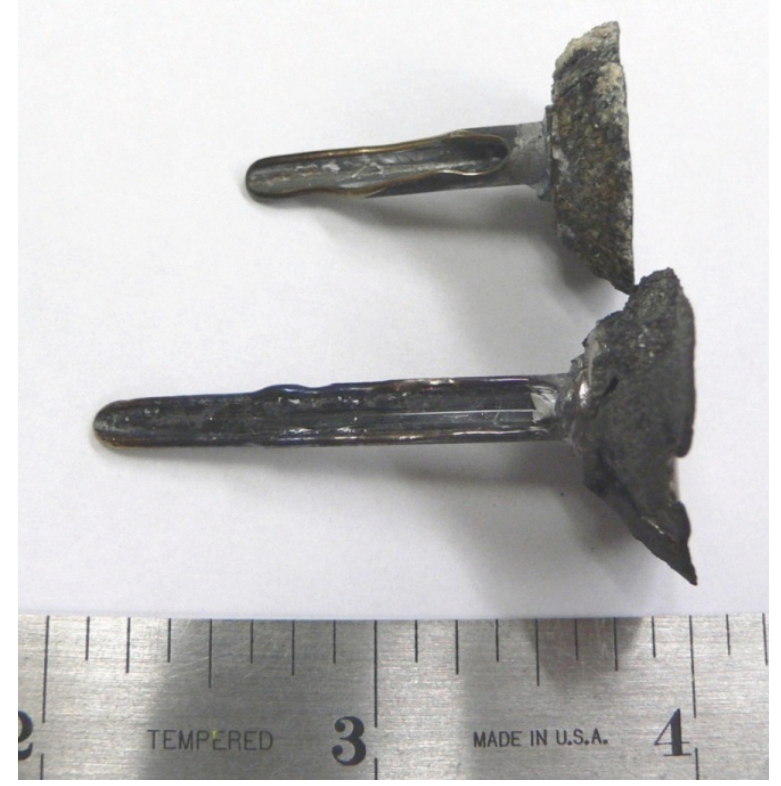

Figure 17. Examples of gravity arc casting products.

\section{Lalb Scale Injection Arc Gasting}

To promote a more uniform flow around the central core, injection arc casting into the mold shown in Figure 16 was investigated. The results from surrogate castings were not satisfactory as adequate flow around the core could not be achieved. As with gravity arc casting, flow around the mold was inconsistent and the resulting pins tended to be short with multiple void sections. This method was abandoned after early testing because of the inconsistent results and extreme operator dependence.

\section{Gravity Gasting Using a Vacuum Pressure Gasting Machine}

Due to the extreme operator dependence of the arc casting process, a commercially available induction heated furnace system typically employed in jewelry casting was procured. A unique feature of this system is the use of separate semi-isolated chambers that house the crucible and mold. Because the chambers are separate and isolated, a pressure differential can be established to increase the flow rate of the material from the crucible into the mold. Additional features of the system included graphite molds that allow for a large "top hat" or riser that provide slow cooling of the fuel pin and pressure relief 
channels to allow gas escape. Despite being able to control temperature, hold times, and pressures, results were similar to those seen in arc casting. Figure 18 shows two examples of the cast material where, once again, material did not flow around the core despite the large riser. Also note the thin strands of material that ran into the pressure relief channels, which demonstrates that adequate super heating was achieved. Although this clearly allowed for high fluid flow, it was resisted by the core or cooled too quickly to flow fully around core.

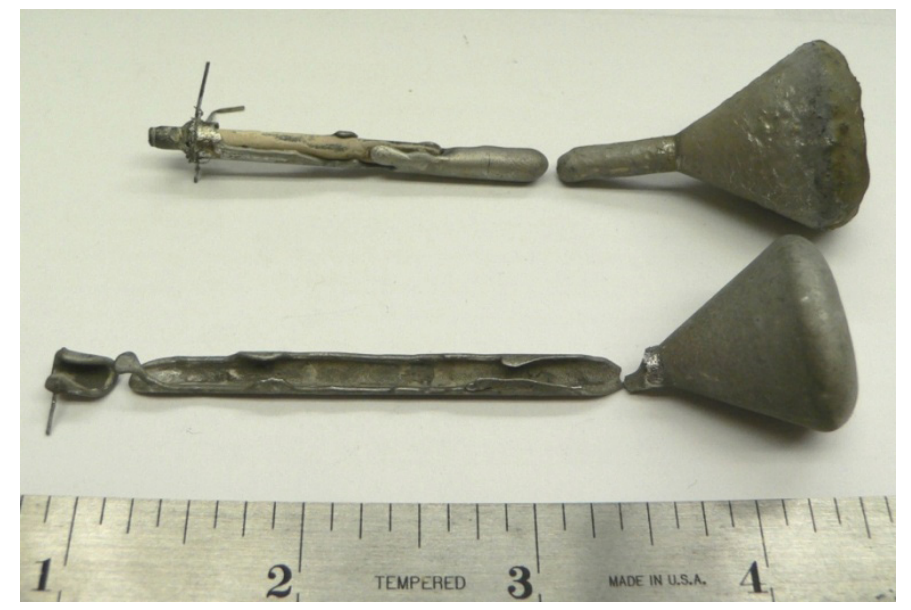

Figure 18. Examples of casting results using a Vacuum Pressure Casting Machine.

\section{Gentrifugal Gasting}

Centrifugal casting was performed with a commercial casting system used for dental and jewelry applications. Here, the charge is inductively heated with the intention that the centrifugal force would be sufficient to fully fill the mold and produce consistent annular pins. The anticipated molten material flow was not completely achieved since void and open sections could be clearly seen in the products.

\section{Open Mold Gravity Are Gasting}

The common failure in all of the casting methods discussed thus far was achieving consistent flow around the quartz or graphite core in order to produce a quality annular cross section. Since the core seemed to inhibit flow, a casting method was attempted that did not employ a core. In the absence of a core, an annular fuel form could be cast through rapid freezing along the mold wall. The fuel annulus size can be controlled by adjusting the temperature of the mold wall. Thus, a glass mold held in a copper tube heat sink resulted in an improved product. Figure 19 shows typical examples of the cast "straws" that have been sectioned in order to examine the inside diameter along the length of the sample. The outside diameters of the straws were quite consistent, varying only $\pm 0.025 \mathrm{~mm}$. The inside diameters were less consistent, varying up to $\pm 0.127 \mathrm{~mm}$, and exhibited some non-concentricity. In an attempt to decrease the inside diameter, or increase the wall thickness, a chilled copper tube was placed around the glass mold. Although the wall thickness was not appreciably increased, the inside diameter was more consistent, however, adequate wall thickness could not be achieved. 


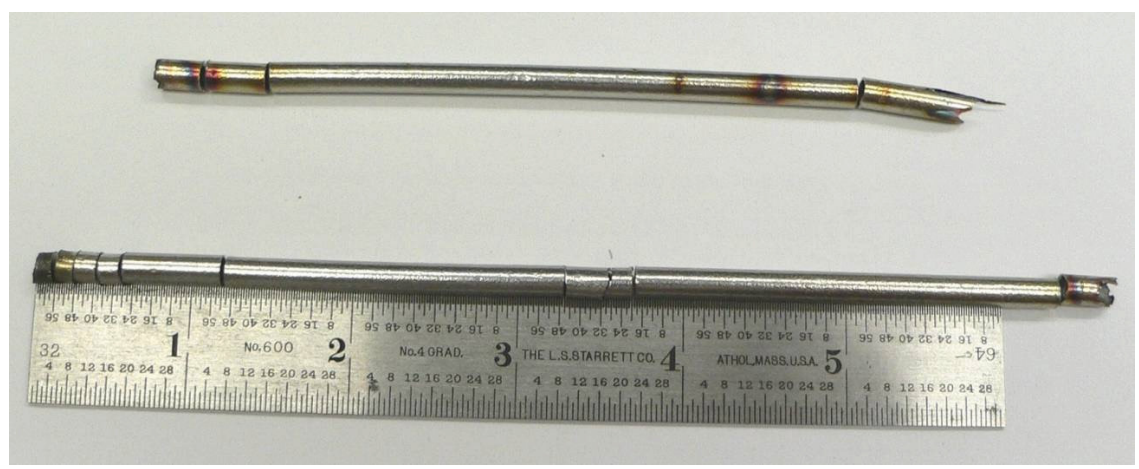

Figure 19. Typical examples of the "straws" cast without use of an inner core.

\section{Gounter Gravity Injection Gasting [CHIC]}

A CGIC technique was used to cast over 130,000 solid fuel pins for the Experimental Breeder Reactor (EBR)-II. Initial testing on pure copper produced annular pins of approximately $100 \mathrm{~mm}$ in length. Continued studies on U-10Mo, U-10Zr, and U-10Zr-(1-4)Pd required certain parameter optimization, including pressure and pre-alloying for mixing. Figure 20 shows examples of the equipment and molds used to cast the annular fuels.

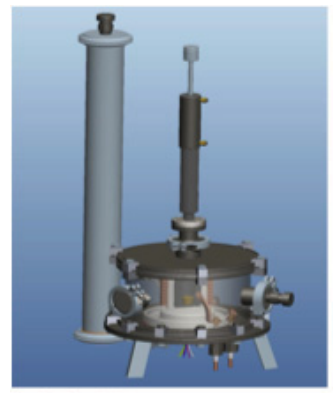

Injection BSC

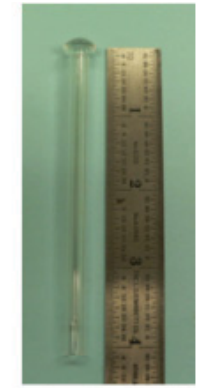

Quartz Annular Mold

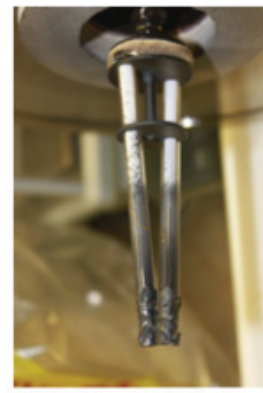

U10Mo (3A-2) Annular Fuel

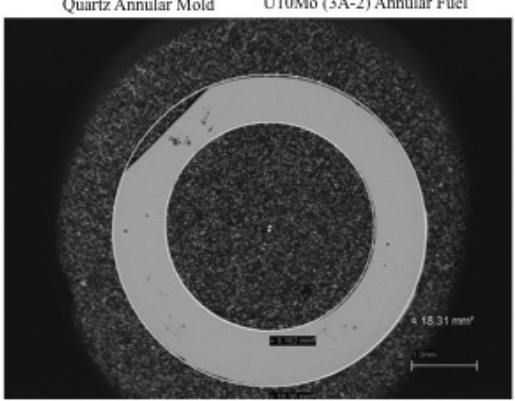

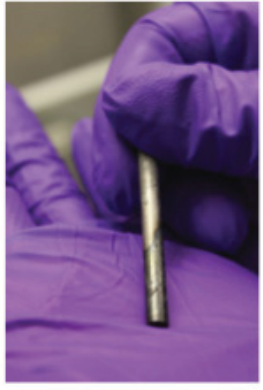

U10Mo (3A-2) Annular Fuel removed from mold

U10Mo (3A-2) Annular Fuel SEM Image (circles are to illustrate concentricity)

Figure 20. Equipment, molds, and example results from the CGIC process development studies.

The U-Zr-Pd alloys presented a unique issue for mixing. Past experience with alloying this composition showed that the palladium appears be repelled from the U-Zr alloy. An initial characterization of the U-Zr-Pd slug microstructure yielded a curious microstructure (Figure 21). The Pd and $\mathrm{Zr}$ formed precipitates that greatly reduced the available $\mathrm{Zr}$ in the $\mathrm{U}-\mathrm{Zr}$ matix. Energy dispersive spectroscopy (EDS) analysis shows the lighter phase to have essentially no $\mathrm{Pd}, 3.5-4 \mathrm{wt} \% \mathrm{Zr}$, and the balance $\mathrm{U}$. The pre-alloying process was modified to produce a product showing significantly less phase segregation. In the case of the U-Mo fuels, the cooling rate of the cast material appeared to have a direct effect on the brittleness of the alloy. Although a higher quality product was produced by adjusting the cooling rate, further studies are needed to optimize the process. 


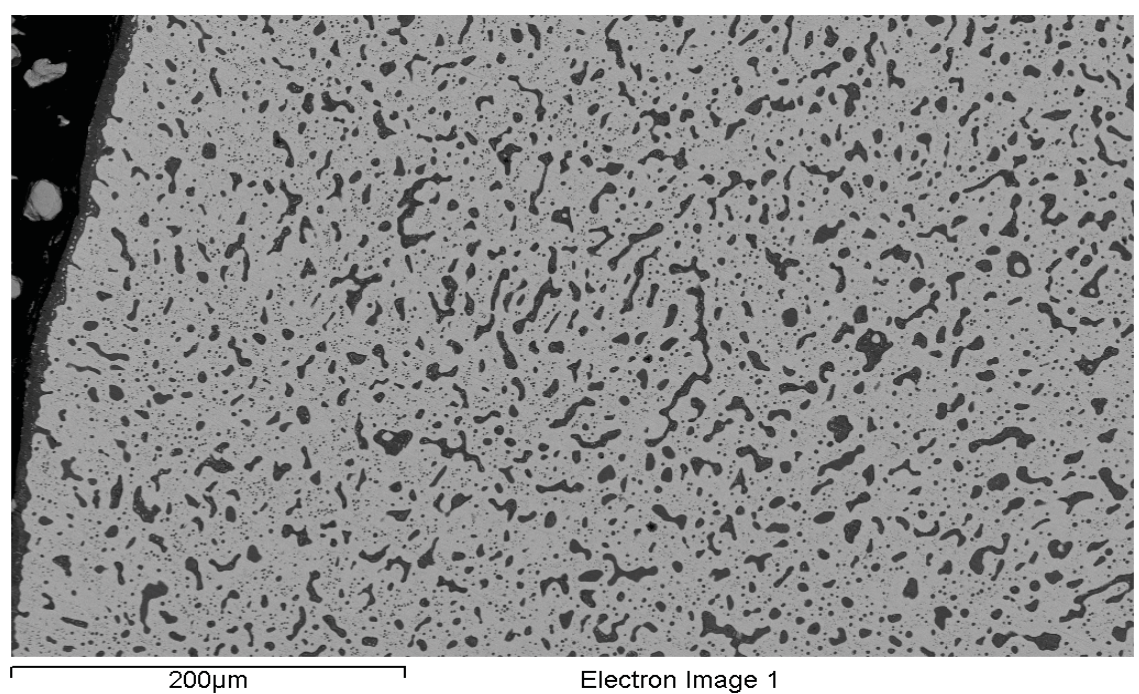

Figure 21. Scanning electron micrograph of the U-Zr-Pd alloy microstructure formed from the CGIC process.

\subsubsection{Glovehox Advanced Gasting System Development and Testing}

Fabrication of the GACS was completed and is currently in mock-up testing. The GACS contains a heated mold and crucible, both inductively heated and independently controlled. Mock-up testing is progressing well with several issues being resolved and small design changes to make glovebox operation more convenient and efficient. Figure 22 and Figure 23 show the furnace in mock-up and the furnace internals, respectively. The GACS will be installed in the Casting Laboratory on the INL site. To make room for the new furnace, an old and obsolete CGIC furnace and associated highly contaminated piping must first be removed. The current status of the installation is the piping was successfully removed with furnace removal to start early FY 2012.

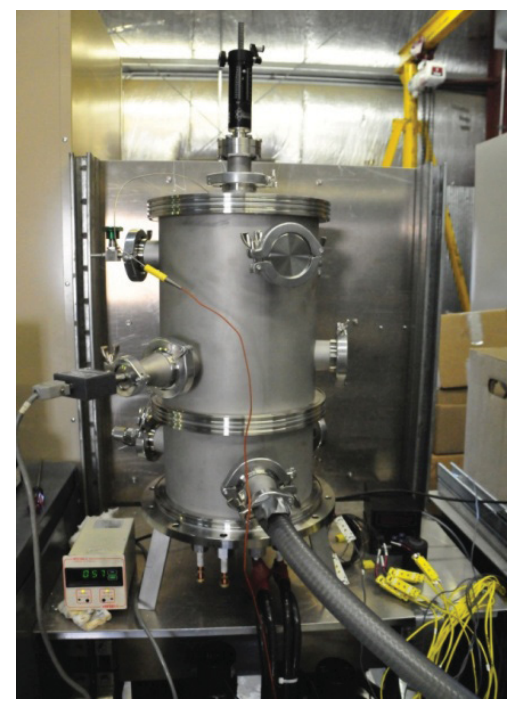

Figure 22. GACS set-up and ready for mock-up testing.

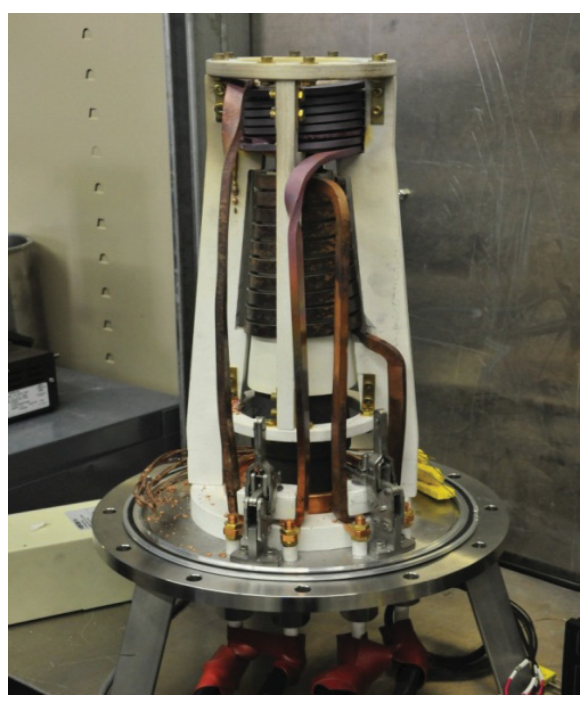

Figure 23. GACS internal components and support structure. 


\subsection{Fuel Fabrication}

T. Hyde, timothy.hyde@inl.gov, INL

The scope of the Fuel Fabrication work package included fabrication of the AFC-3A and AFC-3B irradiation test fuels, fabrication of characterization fuel samples, and fabrication of HFIR irradiation test samples.

\subsubsection{AFC-3A \& AFC-3B Experiment Fabrication}

The fabrication of the AFC-3A and AFC-3B experiments was the primary goal of the fuel fabrication work package during FY 2011. The FCRD AFC-3 irradiation experiments are a continuation of the AFC-1 and AFC-2 scoping level experiments previously irradiated in the ATR. The AFC-3 experiments will consist of metallic and oxide fuels, similar to those in AFC-1 and AFC-2 experiments, as well as other ceramic fuels. The AFC-3 capsule design was modified to limit the content to one rodlet or material test specimen(s) per capsule. The AFC-3 experiments will be irradiated in the ATR Outboard A positions 9-12 using modified hardware designs from the AFC-1 and AFC-2 experiments irradiated in the East Flux Trap (EFT) positions. The AFC-3 experiment series will include multiple tests per year over an estimated ten-plus year duration. Performance data from AFC-3 experiments include irradiation growth and swelling, helium production, helium and fission gas release fractions, fission product and fuel constituent migration, fuel phase equilibria, and fuel-cladding chemical interaction.

INL personnel assembled the experiment capsules, each containing one fueled rodlet suitable for ATR irradiation at the Materials and Fuels Complex (MFC). The capsules were fabricated in the form of a drop-in containment vessel and were fabricated and assembled in accordance with the reference design specifications. AFC-3A and 3B are the first full experiments of the AFC-3 series. The compositions, fuel form, and smear densities of the fuel rodlets are shown in Table 2; note that the compositions are expressed as a weight percent for each constituent element. Several fuel options have an "a" and "b" designation. This indicates that the fuel will be made of two distinct pieces, each of a different composition, which will combine to form the typical 1.5 inch fuel length. Figure 24 through Figure 27 show the final assembly steps for the rodlets and capsules.

Table 2. AFC-3A and $3 B$ Test Matrix.

\begin{tabular}{|c|c|c|c|c|}
\hline Rodlet ID & Fuel Designation & Nominal Composition & Fuel Form & $\begin{array}{c}\text { Nominal Smear } \\
\text { Density }\end{array}$ \\
\hline 3A-R1 & 3A-1 & U-10Mo & Solid & $75 \%$ \\
\hline 3A-R2 & 3A-2 & U-10Mo & Annular & $55 \%$ \\
\hline 3A-R4 & 3A-4 & U-10Zr & Annular & $55 \%$ \\
\hline 3A-R5 & 3A-5A & U-1Pd-10Zr & Solid & $75 \%$ \\
\hline & 3A-5B & U-2Pd-10Zr & Solid & $75 \%$ \\
\hline 3B-R1 & 3B-1 & U-4Pd-10Zr & Solid & $55 \%$ \\
\hline 3B-R2 & 3B-2 & U-4Pd-10Zr & Annular & $55 \%$ \\
\hline 3B-R4 & 3B-4 & U-10Mo & Solid & $55 \%$ \\
\hline 3B-R5 & 3B-5 & U-10Mo & Solid & $55 \%$ \\
\hline
\end{tabular}




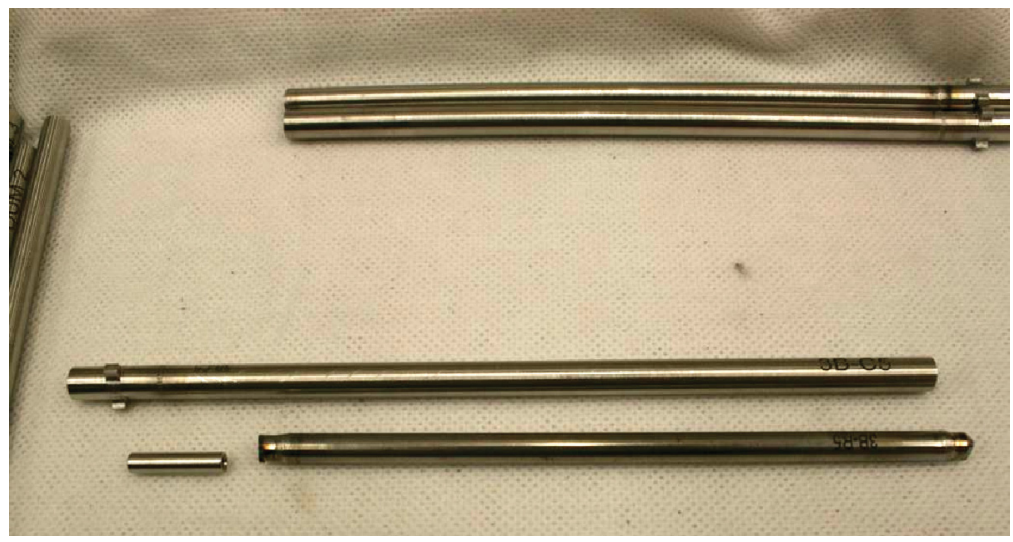

Figure 24. Rodlets and spacer laid out for loading into capsule.

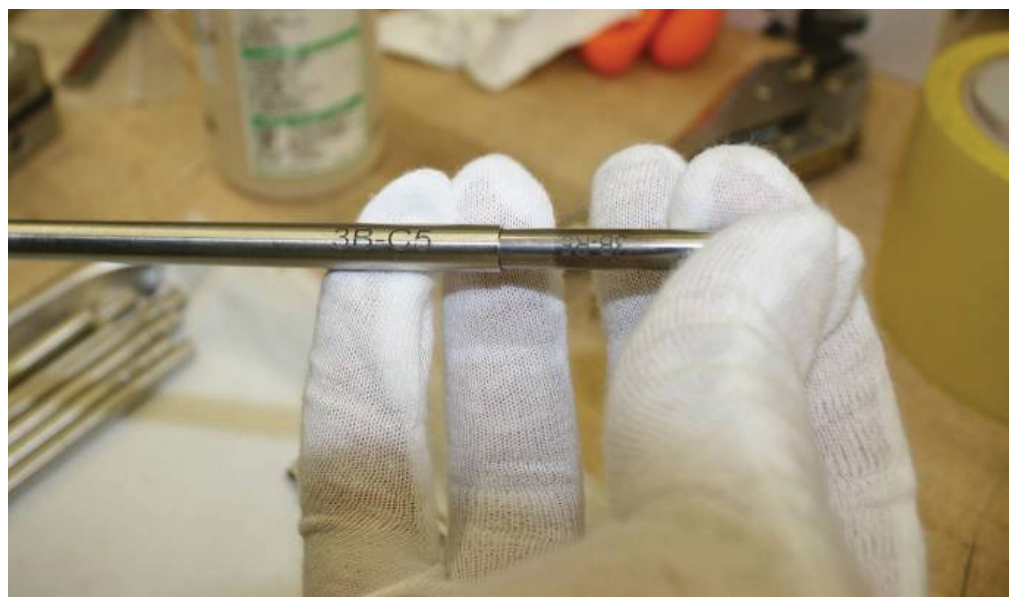

Figure 25. Verifying rodlet serial numbers and inserting rodlet into capsule.

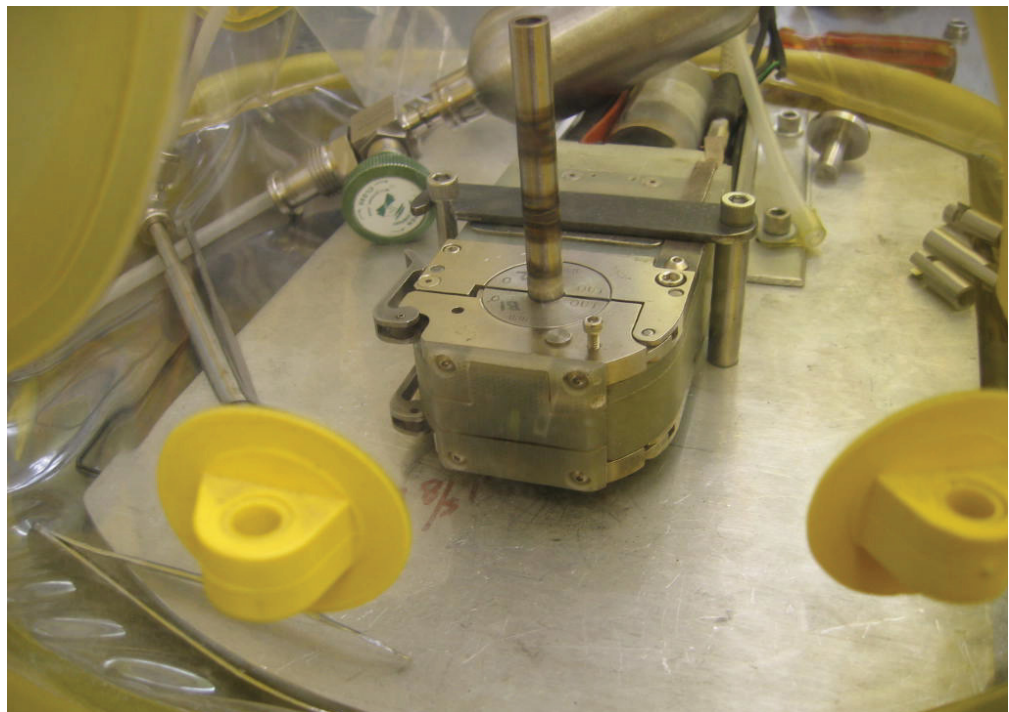

Figure 26. Orbital welding system used for capsule closure weld inside a He glove bag with a test weld piece shown in place. 


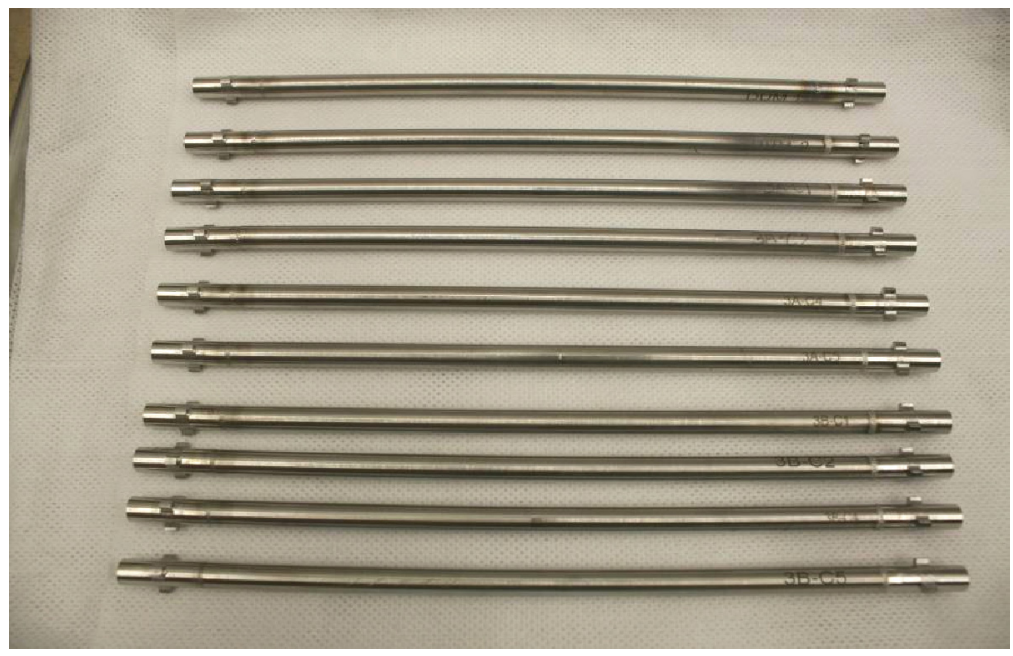

Figure 27. Finished capsules loaded and welded.

\subsubsection{Characterization Castings}

The following alloys and castings were made during FY 2011 to support characterization activities. (This list specifically excludes the fuels cast for reactor insertions.)

1. $82 \mathrm{wt} \% \mathrm{Pu}-18 \mathrm{wt} \% \mathrm{Am}, 4.5 \mathrm{~mm}$ slug

2. $82 \mathrm{wt} \% \mathrm{Pu}-18 \mathrm{wt} \% \mathrm{Am}, 10 \mathrm{~mm}$ slug

3. $85 \mathrm{wt} \% \mathrm{Pu}-15 \mathrm{wt} \% \mathrm{Am}, 4.5 \mathrm{~mm}$ slug

4. $85 \mathrm{wt} \% \mathrm{Pu}-15 \mathrm{wt} \% \mathrm{Am}, 10 \mathrm{~mm}$ slug

5. $90 \mathrm{wt} \% \mathrm{Pu}-10 \mathrm{wt} \% \mathrm{Am}, 4.5 \mathrm{~mm}$ slug

6. $90 \mathrm{wt} \% \mathrm{Pu}-10 \mathrm{wt} \% \mathrm{Am}, 10 \mathrm{~mm}$ slug

7. $95 \mathrm{wt} \% \mathrm{Pu}-5 \mathrm{wt} \% \mathrm{Am}, 4.5 \mathrm{~mm}$ slug

8. $95 \mathrm{wt} \% \mathrm{Pu}-5 \mathrm{wt} \% \mathrm{Am}, 10 \mathrm{~mm}$ slug

9. $98 \mathrm{wt} \% \mathrm{Pu}-2 \mathrm{wt} \% \mathrm{Am}, 4.5 \mathrm{~mm}$ slug

10. $98 \mathrm{wt} \% \mathrm{Pu}-2 \mathrm{wt} \% \mathrm{Am}, 10 \mathrm{~mm}$ slug

11. $90 \mathrm{wt} \% \mathrm{DU}-10 \mathrm{wt} \% \mathrm{Mo}, 4.5 \mathrm{~mm}$ slugs

12. $90 \mathrm{wt} \% \mathrm{DU}-10 \mathrm{wt} \% \mathrm{Zr}, 4.5 \mathrm{~mm}$ slugs

13. $89 w t \% D U-1 w t \% P d-10 w t \% Z r, 4.5 \mathrm{~mm}$ slugs

14. $88 \mathrm{wt} \% \mathrm{DU}-2 \mathrm{wt} \% \mathrm{Pd}-10 \mathrm{wt} \% \mathrm{Zr}, 4.5 \mathrm{~mm}$ slugs

15. $86 \mathrm{wt} \% \mathrm{DU}-4 \mathrm{wt} \% \mathrm{Pd}-10 \mathrm{wt} \% \mathrm{Zr}, 4.5 \mathrm{~mm}$ slugs

16. $85 \mathrm{wt} \% \mathrm{DU}-15 \mathrm{wt} \% \mathrm{Zr}, 4.5 \mathrm{~mm}$ slugs

17. $80 \mathrm{wt} \% \mathrm{DU}-20 \mathrm{wt} \% \mathrm{Zr}, 4.5 \mathrm{~mm}$ slugs

In addition, there were several dozen castings done to develop the right casting parameters and to develop a process to cast annular fuels. 


\subsubsection{HFER Rabbit TEM Sample Fabrication}

Twenty (20) depleted uranium (DU)-10Zr TEM specimens were prepared and characterized for HFIR rabbit testing; Figure 28 shows a typical TEM specimen sample. These samples where received at ORNL on 5/4/11. In addition, there are (20) DU and (20) DU-20Zr samples ready for future shipments. Figure 29 shows typical DU-10Zr sample microstructure.

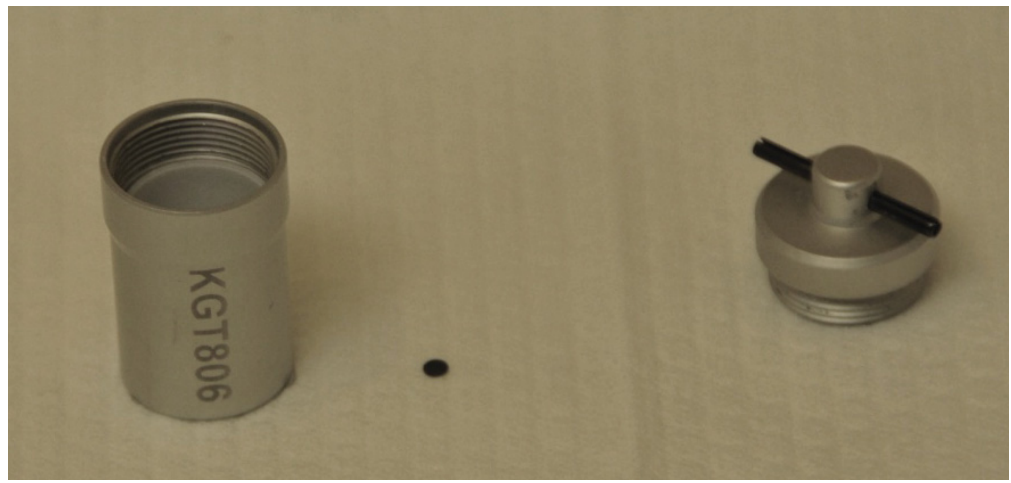

Figure 28. TEM Punch (center) with sample container and lid.

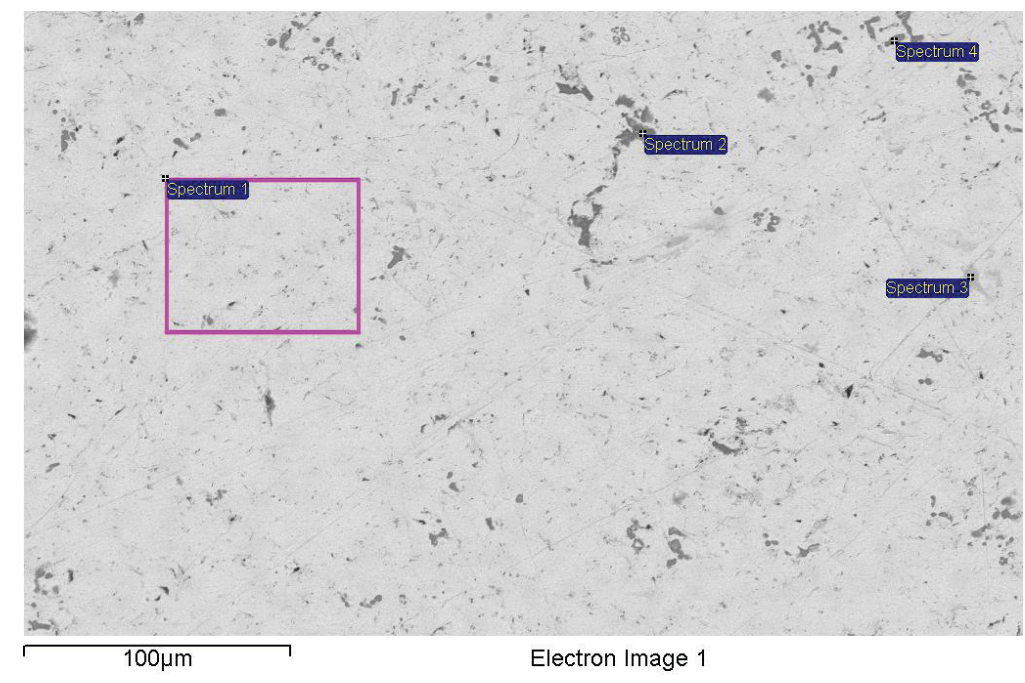

Figure 29. DU-10Zr SEM image showing Zr inclusions.

\subsection{Fuel Characterization}

T. O’Holleran, thomas.oholleran@inl.gov, INL

Significant progress was made in the FASB facility where a new Differential Scanning Calorimeter (DSC), Laser Flash Diffusivity (LFD) instrument, and Dilatometer were installed (Figure 30). The DSC has been run with uranium-bearing materials. The LFD requires an exhaust hood to be installed before running uranium samples. The dilatometer is undergoing some minor plumbing upgrades. The combined data from these instruments is used to determine the thermal conductivity of fuels and materials as a function of temperature, which is important for making safety analyses and for modeling fuel performance. 


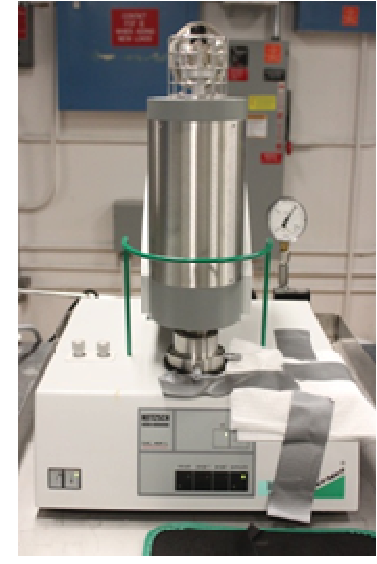

(a)

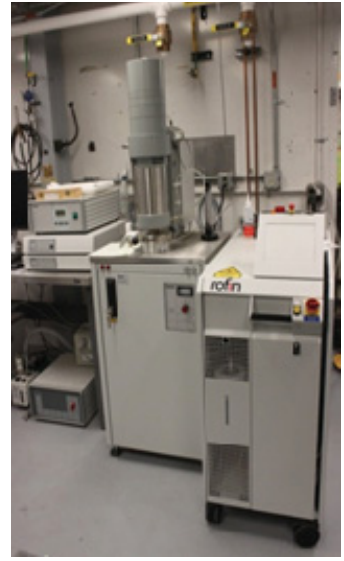

(b)

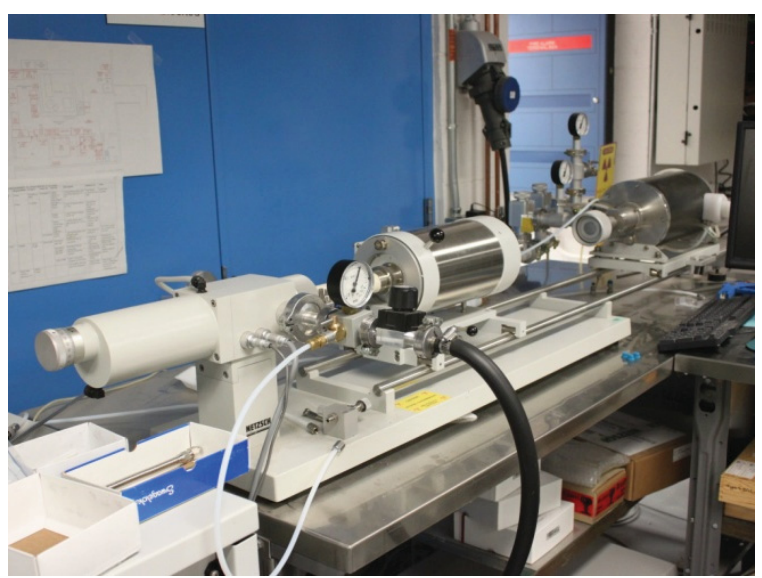

(c)

Figure 30. New instrumentation installed in the Fuels and Applied Science Building (FASB): (a) DSC, (b) LFD instrument, and (c) Dilatometer.

A new PANalytical X-ray diffractometer became operational in FY 2011. A sample holder was designed, fabricated, and tested for handling fresh fuel materials. Ten AFC3 irradiation test samples were analyzed: five U-10Mo alloys and five U-10Zr alloys. Preliminary characterization by SEM and X-ray diffraction (XRD) verified that these castings were acceptable for reactor testing. Figure 31 shows the SEM results for one of the AFC3 U-Zr-Pd alloys.
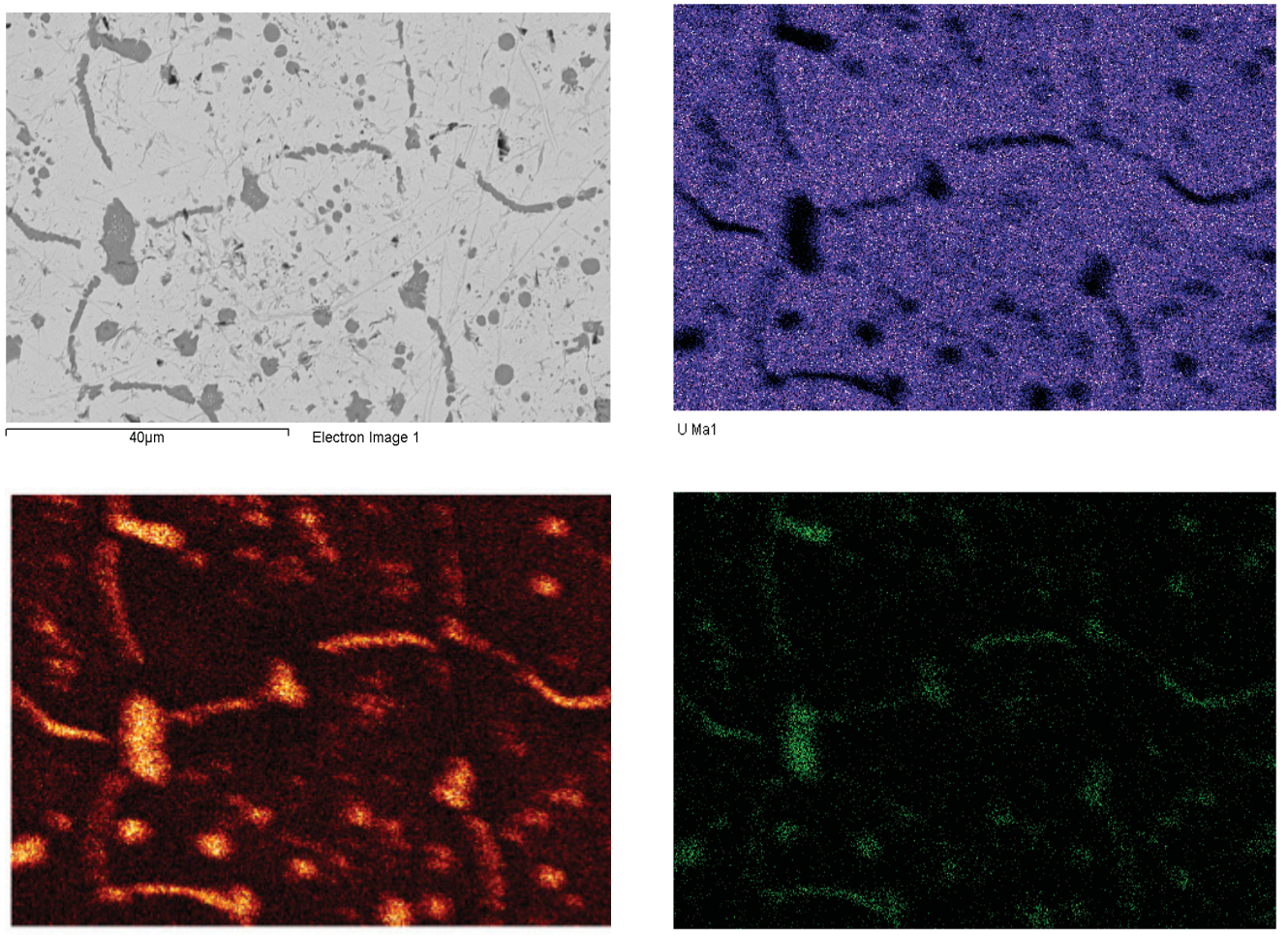

Zr La

PdLa1

Figure 31. Back scattered electron image (upper left) and X-ray maps showing the microstructure with $\mathrm{Zr}$ and $\mathrm{Pd}$ co-located in inclusions. 
The FIB was used to mill lamellae from two diffusion couples, U,Pu-Mo and U,Pu-Zr. This technique is a way of directly preparing samples (lamellae) for TEM. This is the first time this technique has been used to prepare TEM samples from FCRD diffusion couples used to study FCCI. Figure 32 shows the two diffusion couples and locations from which lamellae were obtained. Figure 33 shows representative lamellae from each diffusion couple and Figure 34 shows the mounted lamellae ready for insertion into the TEM. When examined by TEM, these samples will reveal interaction details not previously observable.

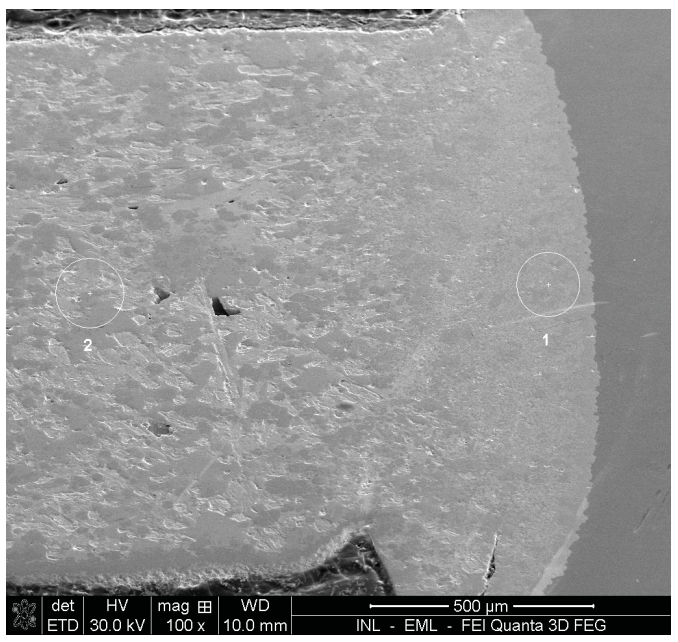

(a)

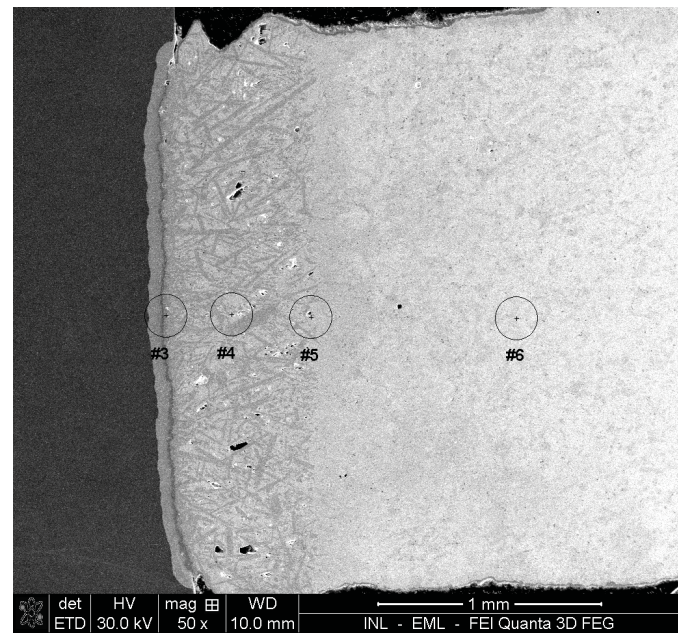

(b)

Figure 32. Secondary electron images showing locations from which lamellae were obtained (circles with $+s$ in the center indicating lamellae locations). (a) The U,Pu-Mo diffusion couple with the U,Pu shown as the lighter region to the left and Mo as the uniform gray region to the right. (b) The U,Pu-Zr diffusion couple with the U,Pu shown as the lighter region to the right and $\mathrm{Zr}$ as the uniform dark region to the left.

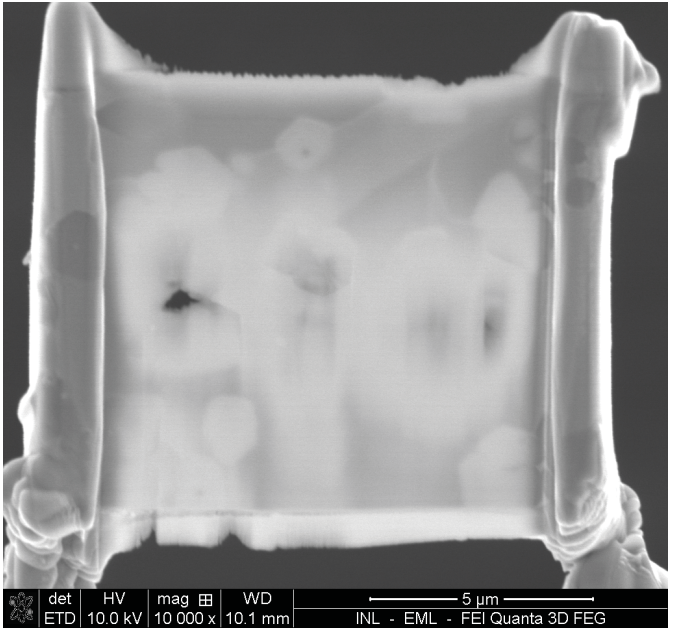

(a)

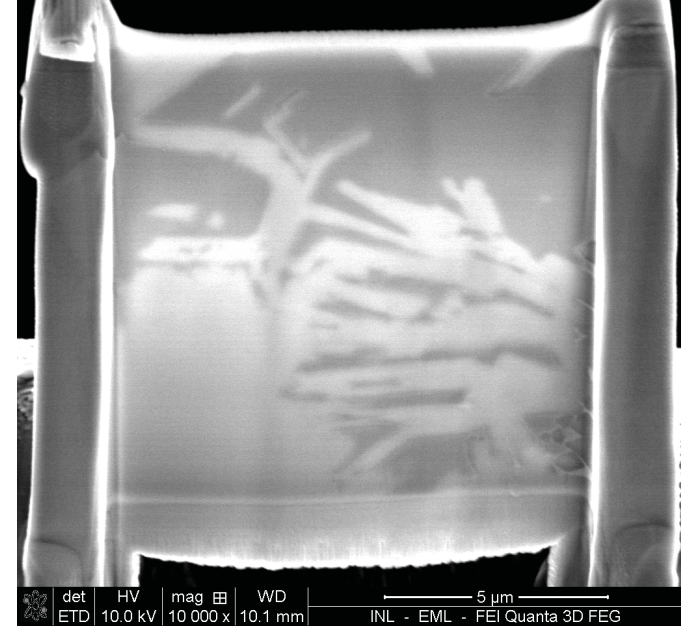

(b)

Figure 33. Secondary electron images showing lamellae from (a) position 2 in the U,Pu-Mo diffusion couple (Figure 32a), and (b) position 4 in the U,Pu-Zr diffusion couple (Figure 32b). 


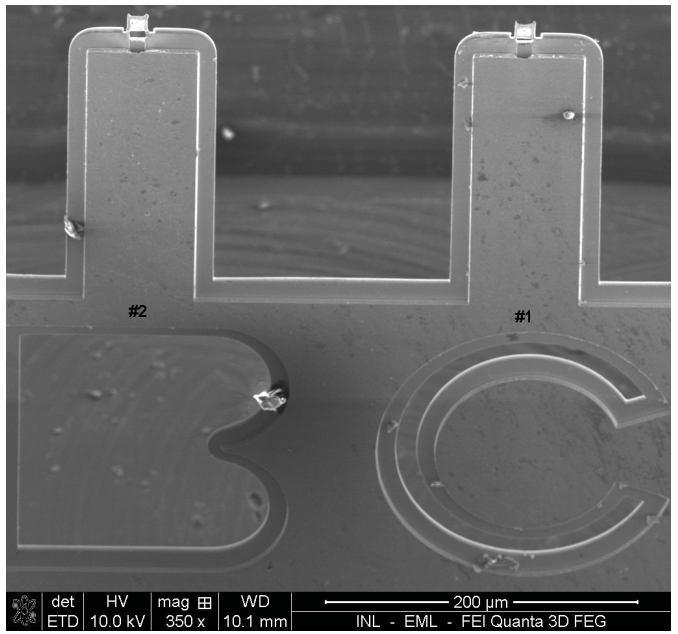

(a)

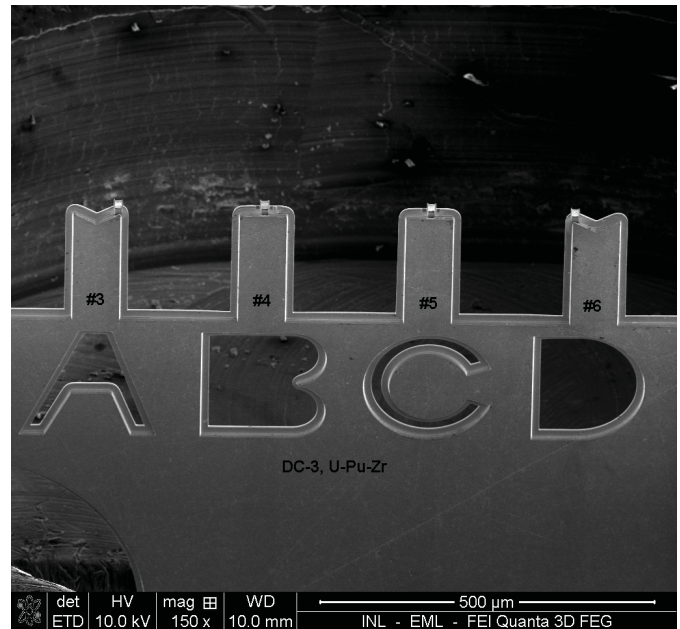

(b)

Figure 34. Secondary electron images showing mounted lamellae ready for insertion in the TEM. The lamellae are the small, light colored rectangular objects welded to the tops of the posts above the large letters. (a) Lamallae from the U,Pu-Mo diffusion couple, and (b) lamellae from the U,Pu-Zr diffusion couple. The numbers at the bottoms of the posts correspond to the positions indicated in Figure 32 from which the lamellae were obtained.

Microstructure characterization efforts during FY 2011 focused on the analysis of two binary $\mathrm{Pu}-\mathrm{Zr}$ alloys: nominal composition of $\mathrm{Pu}-10 \mathrm{wt} \% \mathrm{Zr}$ and nominal composition of Pu-30wt\%Zr. Both as-cast samples and annealed $\left(500^{\circ} \mathrm{C}, 1 \mathrm{wk}\right)$ samples were examined. The objective of this work was to evaluate the approach to equilibrium resulting from this heat treatment as compared to published phase equilibrium data. Each sample consisted of a section approximately perpendicular to the long axis of a fuel rodlet. The annealing operation was performed prior to cutting of the individual slices.

There were no obvious differences between the microstructures in the as-cast and annealed samples of the Pu-10Zr alloy (Figure 35). These images suggest that the samples may contain at least four phases, which can be recognized by their contrast in back-scattered electron (BSE) images. Two of the phases occur as inclusions, while the remaining two make up the matrix. The numbers in Figure 35a give reference to examples of all four phases. Phase 1 inclusions are very dark and contain high concentrations of Si. Phase 2 inclusions are somewhat lighter and contain high concentrations of Zr. Phases 3 and 4 are lighter and contain varying proportions of $\mathrm{Pu}$ and $\mathrm{Zr}$. Because the samples are fine-grained, it is likely that all of the spectra represent more than one phase, making it impossible to reliably determine the composition of any individual phase. 

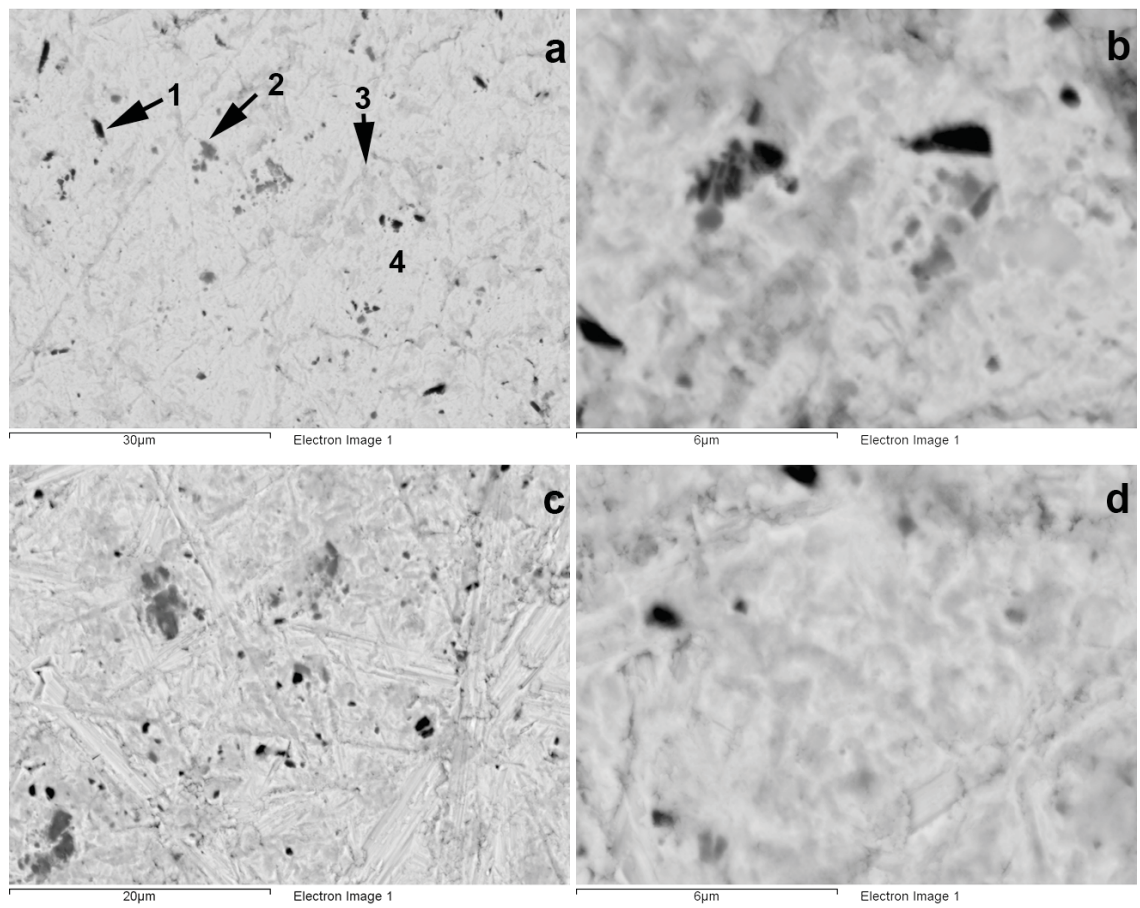

Figure 35. BSE images from as-cast ( $a, c)$ and annealed (b, d) samples of a Pu-10Zr sample (AFC2-B5). Numbers and arrows in a) show what may be four phases. Linear features in (c) are scratches from sample preparation.

Microstructures in as-cast and annealed samples of the $\mathrm{Pu}-30 \mathrm{Zr}$ alloy also appeared similar (Figure 36) and consist of large, dark, dendritic, high-Zr inclusions in a finer matrix. The edges of the high- $\mathrm{Zr}$ inclusions were significantly darker than the interiors in the annealed samples; similar differences in contrast also occurred in the as-cast samples but were less conspicuous. Attempts to identify chemical differences between the material in the interior and at the edges were unsuccessful. Higher-magnification images such as Figure 37 suggest that the difference in contrast between the interior and the edges of the dendritic inclusions might occur because the interiors of the inclusions consisted of a nanocrystalline mixture of two phases, at least one of which is lighter than the material at the edges of the inclusions. However, further investigation (ideally by transmission electron microscopy of a sample produced by FIB) is needed. 

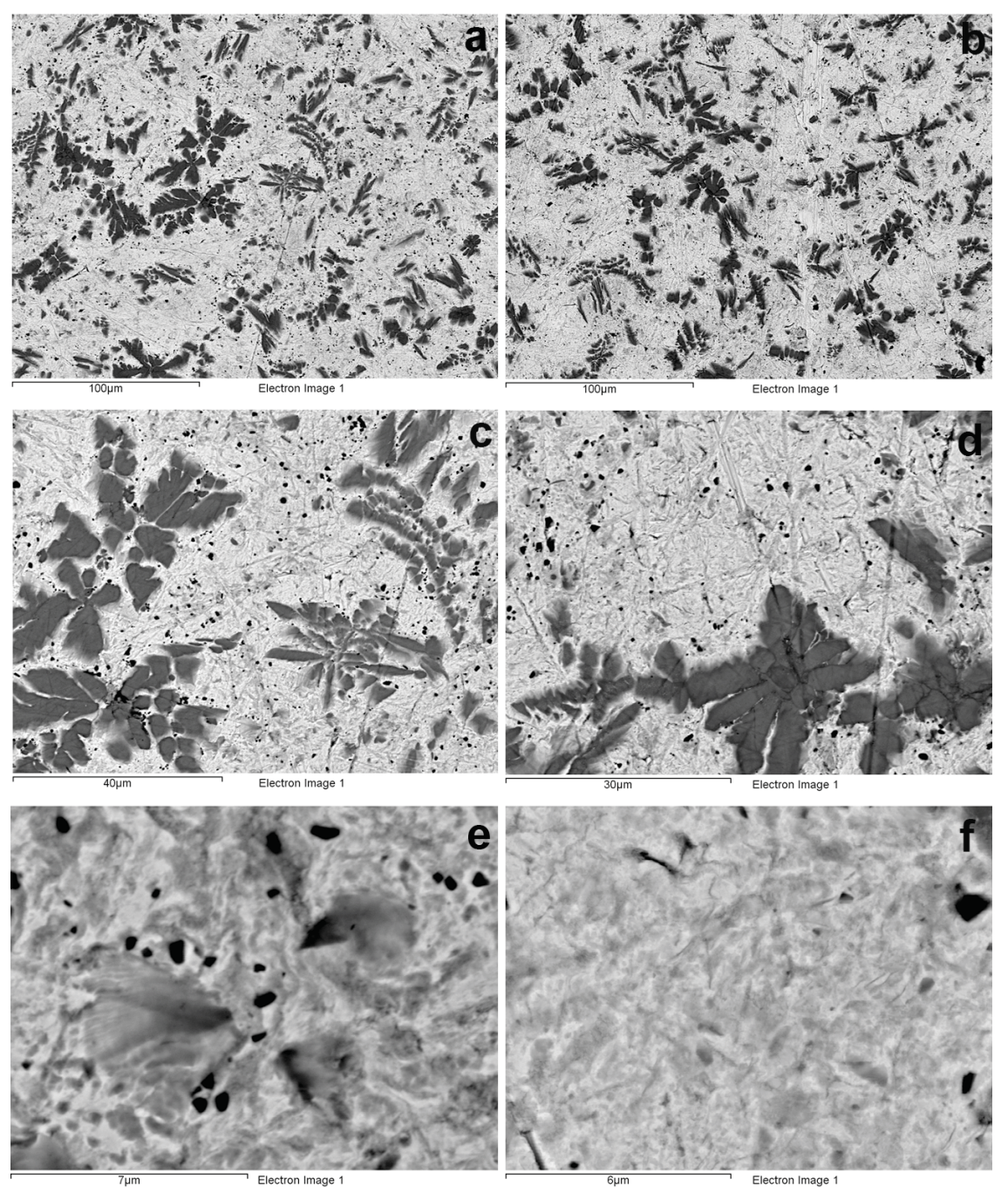

Figure 36. BSE images from as-cast (a, c, e) and annealed (b, $d, f)$ samples of a Pu-30Zr alloy. 


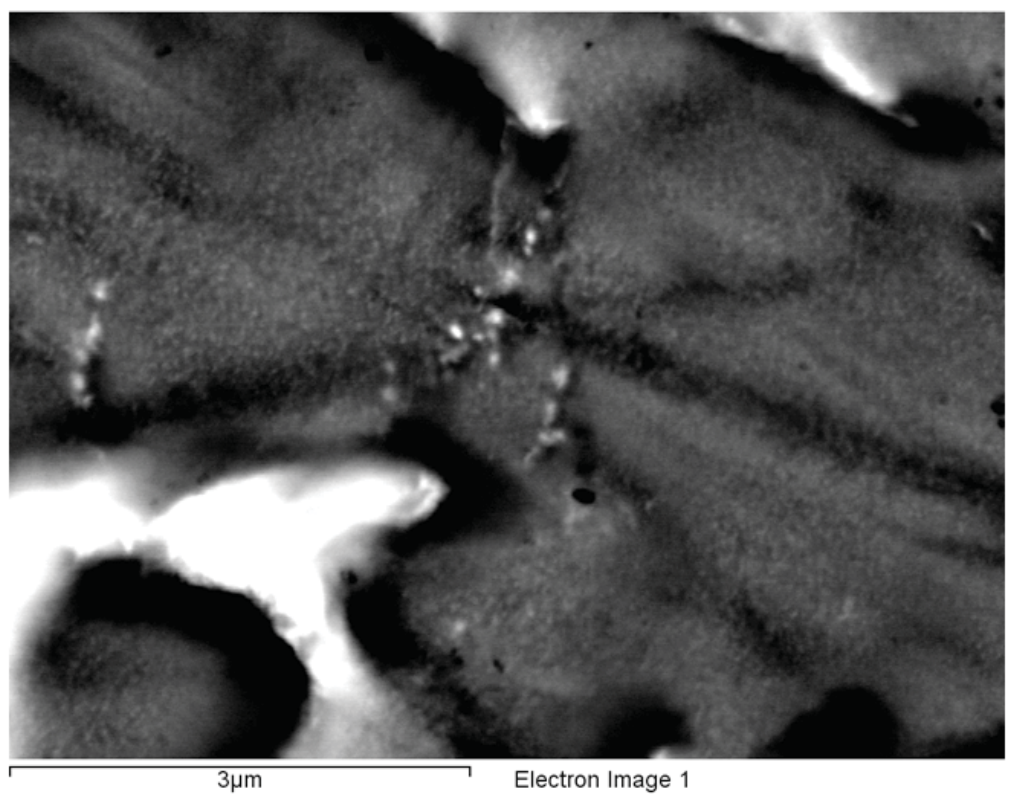

Figure 37. BSE showing details of a high-Zr dendritic inclusion in an as-cast Pu-30Zr alloy. The finely speckled appearance of the interior indicates a nanocrystalline mixture of two phases with significantly different atomic numbers.

Smaller, more euhedral dark inclusions in both as-cast and annealed samples of the Pu-30Zr alloy (e.g., Figure 36e) were high in Si. The matrix in all of the samples showed a fine-scale intergrowth of two phases. Because of the small size of the matrix phases, it was not possible to obtain EDS data from either phase; however, the contrast in BSE images such as those in Figure 36 (which are strongly sensitive to differences in atomic number) suggested that both matrix phases contain $\mathrm{Pu}$ and $\mathrm{Zr}$, but in significantly different proportions.

Further investigation is needed to determine whether the two alloys described here are consistent with the $\mathrm{Pu}-\mathrm{Zr}$ phase diagrams proposed by Okamoto and by Ellinger and Land (ASM Alloy Phase diagrams Center). These phase diagrams indicate that both alloys should be single-phase $\delta$-Pu at $500{ }^{\circ} \mathrm{C}$, but suggest different room-temperature phases. The Okamoto diagram shows only three room-temperature phases: $\alpha-\mathrm{Pu}(<1 \% \mathrm{Zr}), \mathrm{Pu}_{4} \mathrm{Zr}(10-30$ at $\% \mathrm{Zr}$ ), and $\alpha-\mathrm{Zr}$ (whose composition is not shown but appears likely to be $>90 \% \mathrm{Zr}$ ). According to this phase diagram, the $\mathrm{Pu}-10 \mathrm{Zr}$ alloy (which is $\sim 75 \mathrm{at} \% \mathrm{Pu}$ and 25 at $\% \mathrm{Zr})$ at room-temperature equilibrium should be single phase $\left(\mathrm{Pu}_{4} \mathrm{Zr}\right)$ and the $\mathrm{Pu}-30 \mathrm{Zr}(\sim 47 \mathrm{at} \% \mathrm{Pu}$ and 53 at $\% \mathrm{Zr}$ ) should have approximately $1 / 3 \alpha-\mathrm{Zr}$ and $2 / 3 \mathrm{Pu}_{4} \mathrm{Zr}$. The phase diagram proposed by Ellinger and Land (1971) shows five room-temperature compositions: $\mathrm{Pu}_{28} \mathrm{Zr}$ ( $\sim 2$ at $\% \mathrm{Zr}$ ), two distinct ranges for $\mathrm{Pu}_{4} \mathrm{Zr}(\sim 5-13$ and $18-23$ at $\% \mathrm{Zr}), \delta-\mathrm{Pu}(\sim 40-70$ at $\% \mathrm{Zr})$, and $\alpha-\mathrm{Zr}(>88$ at $\% \mathrm{Zr})$. In this case, the $\mathrm{Pu}-10 \mathrm{Zr}$ alloy should contain $\mathrm{Pu}_{4} \mathrm{Zr}$ and $\delta$-Pu, while the $\mathrm{Pu}-30 \mathrm{Zr}$ alloy should be single phase $\delta-\mathrm{Pu}$. 
The experimental data show that neither the $\mathrm{Pu}-10 \mathrm{Zr}$ nor the $\mathrm{Pu}-30 \mathrm{Zr}$ alloy is single-phase and both are far more similar to each other than would be expected from either of the two phase diagrams. Thus, neither alloy appears to be at equilibrium, either at $500^{\circ} \mathrm{C}$ or at room temperature. It remains possible that both alloys were at equilibrium at $500{ }^{\circ} \mathrm{C}$ and that the currently observed microstructures formed during cooling. Experiments with longer annealing times and different cooling rates are needed. Very fine-scale microstructures like those shown in Figure 37 may suggest either the existence of a previously unknown two-phase region at the high-Zr end of the phase diagram or reactions between the high-Zr inclusions and the adjacent matrix. Interpretation of the microstructures in all of the samples requires both crystallographic data and chemical data with higher spatial resolution than can be obtained with SEM. Thus, TEM data collected from samples prepared from selected areas using FIB should proceed.

\subsubsection{Scanning Thermal Diffusivity Microscope}

Four irradiated fuel samples were examined with the STDM whose initial compositions are shown in Table 3. One of these fuels, with initial composition 35U-29Pu-4Am-2Np-30Zr, represents an irradiated version of a previously measured unirradiated fuel. The STDM data for this fuel has been analyzed as discussed below.

Table 3. Initial compositions and positions of irradiated fuel examined with the STDM.

\begin{tabular}{|c|l|}
\hline Composition & Position \\
\hline $40 \mathrm{Pu}-10 \mathrm{Am}-10 \mathrm{~Np}-40 \mathrm{Zr}$ & Rodlet 2 \\
\hline $48 \mathrm{Pu}-12 \mathrm{Am}-40 \mathrm{Zr}$ & Rodlet 1 \\
\hline $35 \mathrm{U}-29 \mathrm{Pu}-4 \mathrm{Am}-2 \mathrm{~Np}-30 \mathrm{Zr}$ & Rodlet 1 \\
\hline $30 \mathrm{U}-25 \mathrm{Pu}-3 \mathrm{Am}-2 \mathrm{~Np}-40 \mathrm{Zr}$ & Rodlet 6 \\
\hline
\end{tabular}

Figure 38 shows an optical microscope image of the irradiated fuel sample, which is unfortunately the only available image at this time. This measurement represents the first comparison of a fuel element from an unirradiated state to PIE at $50 \mu \mathrm{m}$ spatial resolution. In Figure 39, the diffusivity data obtained from the irradiated fuel sample is plotted. On average, the thermal diffusivity was found to be about $20 \%$ lower than in the unirradiated fuel. Unlike in the unirradiated fuel, there does not appear to be a higher thermal diffusivity nearer the center of the sample than at the edges. The number of measurements across the irradiated sample is much larger than that in the unirradiated fuel, one measurement about every 25 $\mu \mathrm{m}$. Out of a total of 200 measurements, 179 were readable, with most of the remaining 21 showing low diffusivity values that could not be reliably quantified. 


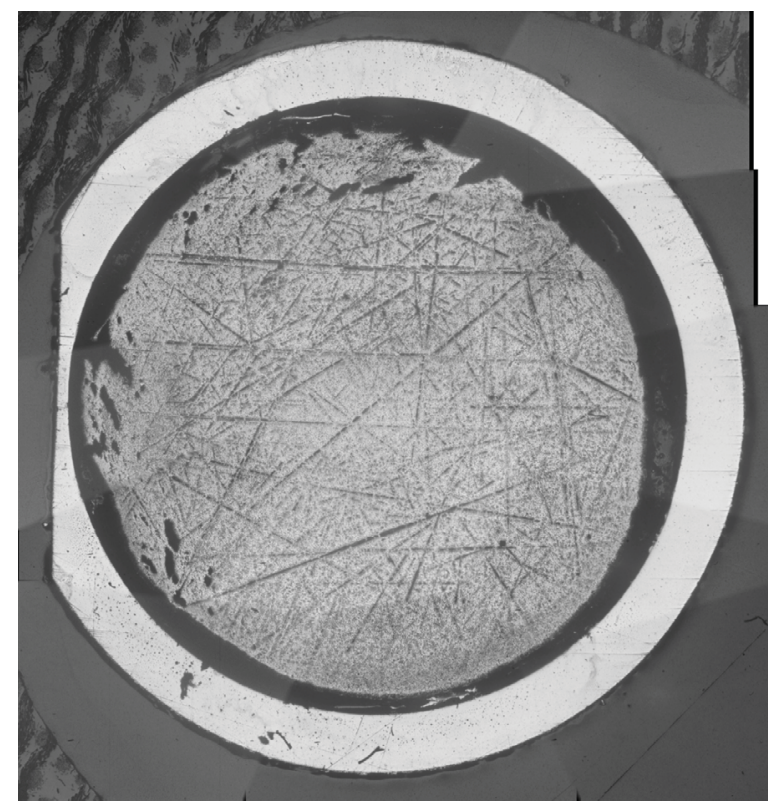

Figure 38. Optical image of the irradiated fuel sample (image courtesy of Heather Chichester).

\section{Scan Across Fuel Pin}

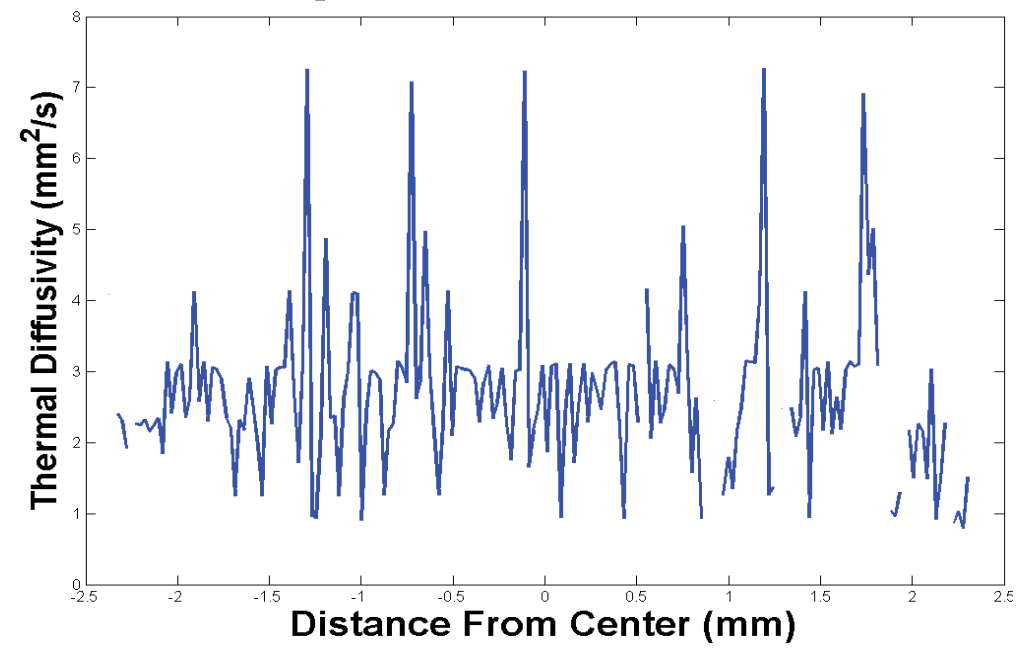

Figure 39. Irradiated fuel diffusivity results from the STDM.

\subsection{Fundamental Fission Products Behavior}

R. Mariani, robert.mariani@inl.gov, INL

Porosity in nuclear fuels is a direct consequence of fuel expansion as it undergoes radial (to fill the space between the fuel and cladding wall) and axial swelling. Transport and diffusion studies involving fuels and engineering materials are usually conducted with fully dense substrates. While these studies and data are very important for characterizing fuel behavior, transport rates are typically much higher on grain boundary surfaces and pore surfaces. This work package intends to identify the potential for higher transport rates with particular focus on metallic fuels. The potential differences arising from the different transport rates and mechanisms in porous fuels are illustrated in Figure 40 and emphasize the need for a better understanding in order to correctly model the fuel behavior. 
To characterize transport in porous fuels at elevated temperatures, it is necessary to (1) prepare porous bodies, (2) ensure the morphology is reasonably stable for the duration of the test (to simplify analysis of the results), and (3) prepare diffusion couples with materials (e.g., Fe, Nd) that are known to undergo transport (based on PIE). A desired feature concerning the porosity is its connectedness.Figure 40 illustrates the possible differences between open (i.e., connected) and closed porosity. Also desirable is maximal, or at least consistent, interfacial contact between the porous fuel body and the test substrate

The development of a method for preparing porous U-10Zr samples was successful. Here, powdered U-10Zr was produced using existing "atomization" equipment. A sizable fraction of the powder exhibited a flake-like morphology, rather than a spherical morphology, which, in fact, was more desirable for producing open-pore bodies upon sintering. The powder was subsequently sintered to less than full density using electrical resistance for short times (minutes) at temperatures estimated to be $850-900^{\circ} \mathrm{C}$. The resulting porosity (Figure 41 ) was verified to be open using a static vacuum technique and it is comparable to the porosity exhibited on the peripheral surface of EBR-II fuels at 2 at\% burnup. Near-term efforts are directed toward preparing the first diffusion couple with a porous fuel alloy.

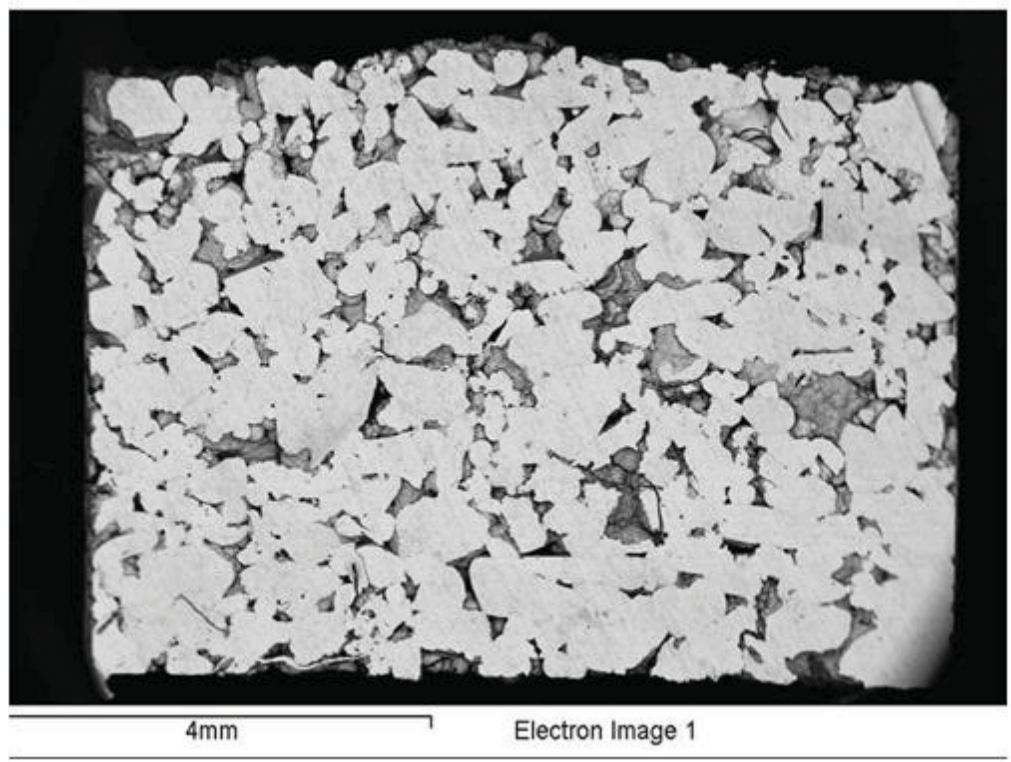

Figure 41. Porous U-Zr pellet produced with approximately 20-30\% porosity, by image analysis. 


\title{
4.7 Fundamental Thermophysical Properties
}

\author{
T. O’Holleran, thomas.oholleran@inl.gov, INL
}

Experiments were conducted to establish methods for handling TRU samples at the University of Nevada-Las Vegas (UNLV) for measurements with the Physical Properties Measurement System (PPMS). The PPMS is an instrument system manufactured by Quantum Design and used to measure fundamental thermophysical properties such as heat capacity with high accuracy. Figure 42 shows the instrument used for these measurements at UNLV.

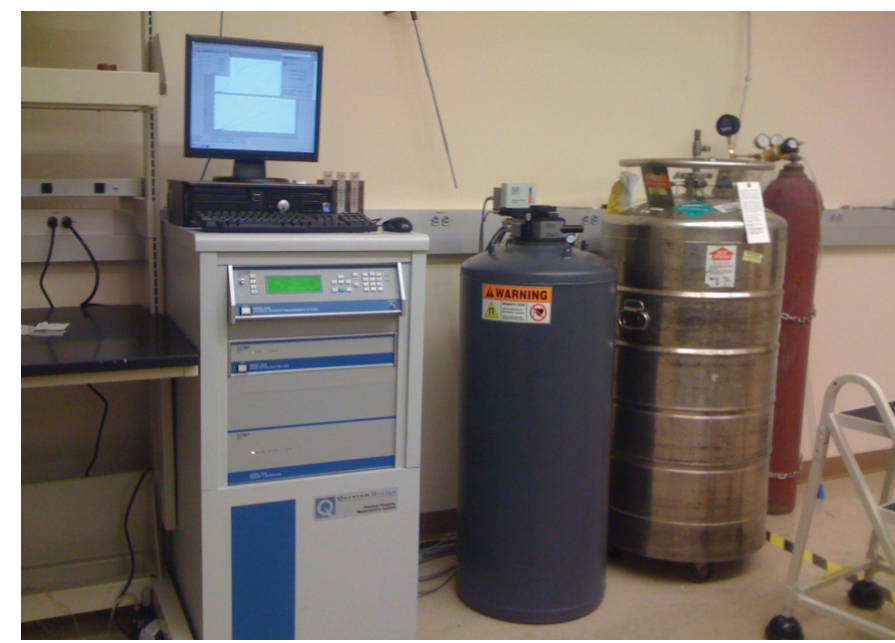

Figure 42. The PPMS at UNLV. During measurement, the sample resides in the dark gray Dewar near the center of the photograph.

Initial experiments demonstrated the system's ability to accurately measure heat capacity (as determined from standard reference materials) and to distinguish changes in heat capacity resulting from small changes in composition. Figure 43 shows the measured change in the heat capacity of uranium resulting from the addition of 3 atomic percent titanium.

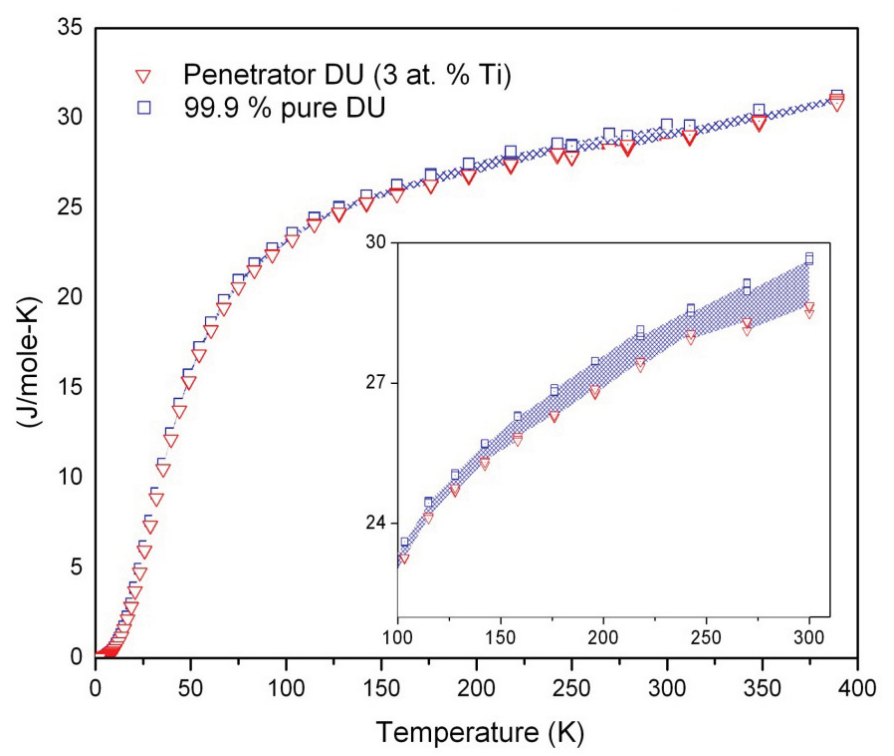

Figure 43. Measured heat capacity of uranium compared to the measured heat capacity of uranium from a DU penetrator containing 3 at\% Ti as a function of temperature. The difference between the two is easily distinguished in the 100 to $400 \mathrm{~K}$ range. 
Efforts in FY 2011 focused on two objectives:

1. Determine the minimum mass of material needed to produce reliable results.

2. Develop a practical method of confining highly radiotoxic materials before and during measurement so that they can be safely handled in the facilities at UNLV.

Experimental results indicate that the minimum mass of sample required to produce reliable results is about $5 \mathrm{mg}$, a quantity small enough to significantly reduce the radiological hazard.

Three methods of confining radioactive materials were investigated. The first method uses a small aluminum container and lid to confine the sample, the second method (a method used at ITU) encapsulates the sample in a thermally conductive epoxy resin, and the third method involves physical deposition of tungsten on the sample to produce a thin barrier layer. The first method, employing an aluminum container, showed undesirable deviation in the thermal conductivity vs. temperature curve. This deviation may be due to experimental factors such as lid decoupling, timing of the thermal pulse, and/or poor thermal contact between the sample and the aluminum cup. The second method, using thermally conductive epoxy, gave generally poor results attributed to difficulties achieving a uniform mixture of sample and epoxy. The sources of error in both methods are under investigation. The third method, deposition of a tungsten layer, was evaluated with a sputter coating process on a representative substrate. Tungsten coatings are attractive for this application because they are highly refractory, inert to the actinide bearing materials of interest, offer the potential to completely encapsulate the sample (thus mitigating radiological hazards), and prevent the loss of volatile materials from the sample during high temperature measurements. Conventional methods of depositing tungsten coatings on substrates are difficult to apply and often involve high temperatures that would be unacceptable for this application. Experiments with sputter coating showed that tungsten coatings could be deposited, but deposition rates were rather low, at only $400 \mathrm{~nm} / \mathrm{hr}$. Figure 44 shows an electron micrograph of one of these coatings deposited on a glass slide. While the coating pulled away from the substrate (presumably during mounting in a met mount for polishing), the coating did not break up, indicating good mechanical strength. Other methods of depositing tungsten coatings at higher rates are being investigated.

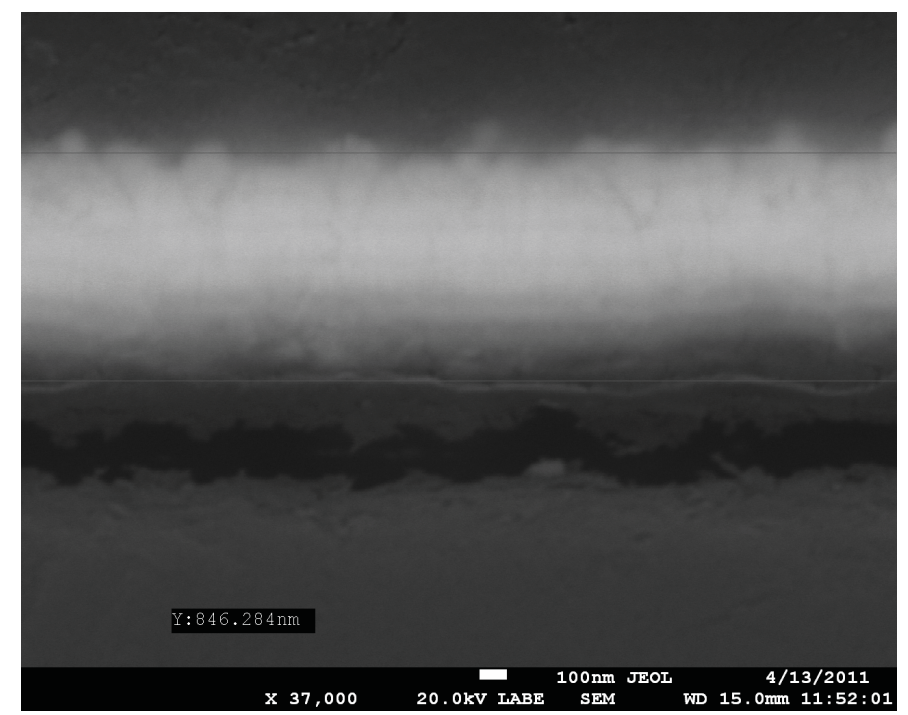

Figure 44. Back scattered electron image of a sputtered tungsten coating deposited on a glass slide. The coating is the bright band across the image. The coating thickness as indicated in the inset is $846 \mathrm{~nm}$. The coating was deposited in six steps, resulting in the vague banding in the image. A crack is visible in the glass substrate below the coating where the coating pulled away (presumably when potted to produce the met mount for polishing) taking a layer of the glass slide with it. This indicates good mechanical strength and adhesion. 
A fourth method has been proposed that combines the aluminum cup and lid with an epoxy encapsulant. This method provides double confinement for the sample and so would be desirable for highly radiotoxic materials such as actinides. This method is currently under investigation.

\title{
4.8 Fundamental Element Redistribution Studies
}

\author{
M. Okuniewski, maria.okuniewski@inl.gov, INL
}

The phenomenon of element redistribution in metallic $\mathrm{U}-\mathrm{Zr}$ and $\mathrm{U}-\mathrm{Pu}-\mathrm{Zr}$ based fuels during reactor irradiation is well documented and results from the significant radial thermal gradient established. The objectives of this work package are to develop an experimental apparatus specifically designed and built to perform thermal transport experiments (i.e., high temperature anneal under a large temperature gradient) and examine the constituent redistribution in U-Zr fuel alloys after the thermal transport heat treatment. The findings of this research will contribute to the basic understanding of the atomic diffusion process by providing a fundamental materials parameter, the heat of transport. Ultimately, this is envisioned to lead to a better design of metallic nuclear fuels and cladding systems.

Significant improvements to the thermal transport apparatus have resulted in the ability to achieve temperatures of up to $1000^{\circ} \mathrm{C}$ and thermal gradients of about $220^{\circ} \mathrm{C} / \mathrm{cm}$ (at the highest temperature ranges). These improvements included a new atmosphere gas gettering-system to reduce the degree of reaction with the furnace atmosphere, a higher temperature furnace, and an oxygen sensor. The result is a more robust high temperature experimental apparatus.

The initial experimental results from thermal transport in the U-Zr system came from a U-10 wt. $\% \mathrm{Zr}$ (U-10Zr) alloy rod (11.8 $\mathrm{mm}$ long and $4.5 \mathrm{~mm}$ diameter) annealed under a temperature gradient of $222^{\circ} \mathrm{C} / \mathrm{cm}\left(\mathrm{T}_{\text {hot }}=1014^{\circ} \mathrm{C}\right.$ and $\left.\mathrm{T}_{\text {cold }}=752^{\circ} \mathrm{C}\right)$ for 348 hours. Throughout this anneal, the oxygen content remained below $3 \mathrm{ppm}$ and the temperature fluctuation never exceeded $\pm 5^{\circ} \mathrm{C}$.

Microstructural and compositional analyses indicated that there was constituent migration under the temperature gradient during the thermal treatment of the specimen. However, an excessive amount of observed Zr-rich precipitates found both before and after the thermal transport test inhibit a clear interpretation of the data. It appears the redistribution behavior of $\mathrm{Zr}$ has a complex dependence on the presence of the Zr-rich precipitates and their variation as a function of the region exposed to a temperature gradient. Clearly, there is an important interplay between thermodynamics (e.g., solubility and cooling rate) and kinetics (e.g., nitridation/oxidation, diffusion of gaseous species in solution and thermal transport).

The configuration of the experimental thermal transport set-up was further evaluated for operation at higher temperatures $\left(\sim 1100^{\circ} \mathrm{C}\right)$ with larger thermal gradients, $\left(\sim 300^{\circ} \mathrm{C} / \mathrm{cm}\right)$. Additional improvements to the experimental set-up were focused on decreasing the oxygen content down to ppb-levels with additional inlet gas oxygen/nitrogen gettering furnaces, a robust gettering system within the thermal transport rig, and scavenger systems.

To accompany the experimental results and assist in experimental analyses, a phase field model was developed for single phase (bcc- $\gamma$ ) and two-phase $(\gamma+\beta) \mathrm{U}-\mathrm{Zr}$ alloys. These models are based on a previously developed model for hypothetical alloys (Mohanty, Guyer, Sohn, J. Appl. Phys., 2009) but utilizes a "true" thermodynamic description of the U-Zr alloy (i.e., CALPHAD based) and atomic/chemical mobility based on literature values. The constituent redistribution within the single $\gamma$-phase along with a relevant parametric study of heat of transport was completed and published, a two-phase $(\gamma+\beta)$ model was developed (Bush, Okuniewski, Sohn, Unpublished, 2011) and a parametric study is being carried out. Upon completion of experimental work, wherein relevant thermo-kinetic coefficients, including the heat of transport for various phases in the U-Zr system, will be determined, these models will serve to validate the experimental results and to identify further critical experiments. 


\title{
4.9 Fundamental FCCI Studies
}

\author{
B. Sencer, bulent.sencer@inl.gov, INL
}

The objective of this work package is to provide an understanding of thermally-activated and separate irradiation effects on multicomponent multiphase diffusion and relevant microstructural evolution within the U-Zr-rare earth (RE) and U-Mo fuel alloy systems in contact with elements or alloys within the Fe-Ni-Cr cladding alloy system. The specific tasks and deliverables will provide critical thermokinetic data and microstructural information and validation for multiscale modeling of the FCCI.

TAMU and the University of Central Florida (UCF) are collaborators on this project. $\mathrm{Mo} / \mathrm{Fe}, \mathrm{Zr} / \mathrm{Fe}$, $\mathrm{Mo} / \mathrm{Zr}, \alpha-\mathrm{U} / \mathrm{Fe}, \beta-\mathrm{U} / \mathrm{Fe}, \mathrm{U} / \mathrm{Mo}, \mathrm{U}-10 \mathrm{Zr} / \mathrm{Fe}, \mathrm{Mo} / \mathrm{Fe}-15 \mathrm{Cr}, \mathrm{Mo} / \mathrm{Fe}-15 \mathrm{Cr}-15 \mathrm{Ni}, \mathrm{Zr} / \mathrm{Fe}-15 \mathrm{Cr}$, and $\mathrm{Zr} / \mathrm{Fe}-15 \mathrm{Cr}-15 \mathrm{Ni}$ diffusion couples have been prepared and analyzed for thermally activated mass transport characteristics. Diffusion couples between $\mathrm{Fe}, \mathrm{Mo}, \mathrm{Zr}, \mathrm{Ce}, \mathrm{Nd}$ and $\mathrm{Ce}-50 \mathrm{Nd}, \mathrm{Fe}-15 \mathrm{Cr}$, and $\mathrm{Fe}-15 \mathrm{Cr}-15 \mathrm{Ni}$ alloys as well as pre-bonded $\mathrm{Fe} / \mathrm{Zr}$ and $\mathrm{Mo} / \mathrm{Zr}$ diffusion couples were irradiated with heavy ions (Fe) to $100 \mathrm{dpa}$ at $450^{\circ} \mathrm{C}$ and $650^{\circ} \mathrm{C}$. A FIB has been used to prepare TEM samples from different regions of several diffusion couples ( $\mathrm{Fe} / \mathrm{Zr}, \mathrm{Fe} / \mathrm{Mo})$ that were irradiated to 100 dpa with heavy ions $(\mathrm{Fe})$. A number of kinetic and diffusion properties (U/Mo, U-10Zr/Fe, U/Fe, etc.) have been calculated. Some of the highlights of the many preliminary results are presented below.

\section{Mo vs. Fe and Fe Alloys}

A series of Fe vs. Mo diffusion couples were assembled and annealed at $750,850,900$, and $1000^{\circ} \mathrm{C}$ for $30,15,30$, and 15 days, respectively. Backscatter electron micrographs with the corresponding concentration profiles of the interdiffusion zone developed in the $900^{\circ} \mathrm{C}$ couple is presented in Figure 45 . It appears that two intermetallic layers have developed which, based on the binary phase diagram, are the $\mu$ and $\lambda$ phases. Another distinct layer is present in the $850^{\circ} \mathrm{C}$ couple with a composition of approximately 96 at.\% Fe and 4 at.\% Mo. This distinction could be a result of the diffusion coefficient being extremely sensitive to the Mo composition. Also, the Mo composition may affect the magnetic transition temperature of $\mathrm{Fe}$, which could play a role in the diffusion process at this temperature.
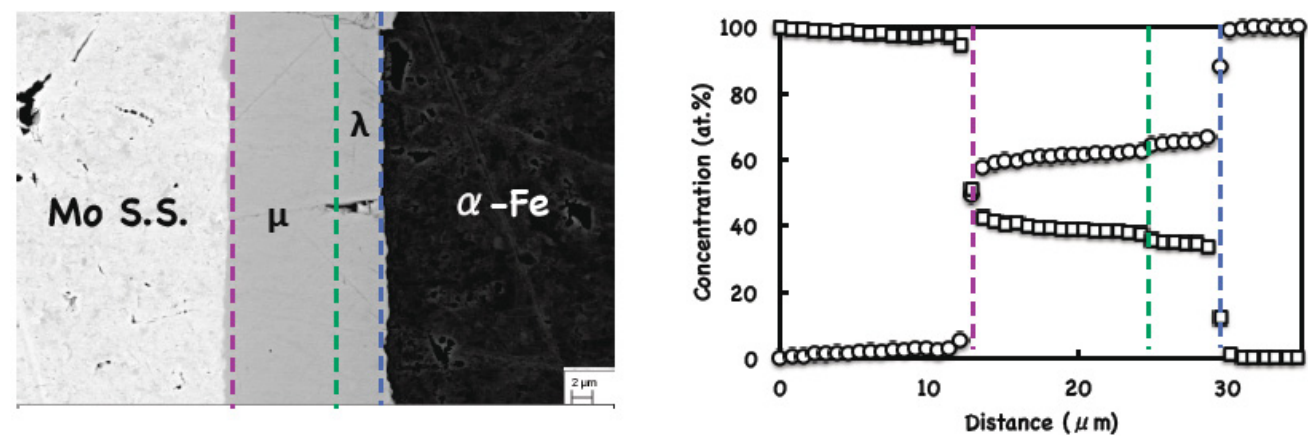

Figure 45. Backscatter electron micrograph and concentration profile of Mo vs. Fe diffusion couple annealed at $900^{\circ} \mathrm{C}$ for 30 days.

\section{Zr vs. Fe and Fe Alloys}

Several different diffusion couples in this series have been assembled and characterized. One selected result is presented in Figure 46 shows the diffusion couple between $\mathrm{Zr}$ and $\mathrm{Fe}$ annealed at $850^{\circ} \mathrm{C}$. Here, two different intermetallic layers developed, likely $\mathrm{Fe}_{2} \mathrm{Zr}$ and $\mathrm{Fe}_{23} \mathrm{Zr}_{6}$ based on the EDS compositions and the phase diagram. The variation in intermetallics that developed is thought to be a result of the allotropic transformation of $\mathrm{Zr}$, but further work needs to be performed to verify this. Also, there is a layer of $\beta-\mathrm{Zr}$ with some solubility for Fe while $\alpha-Z r$ has no solubility for Fe. 

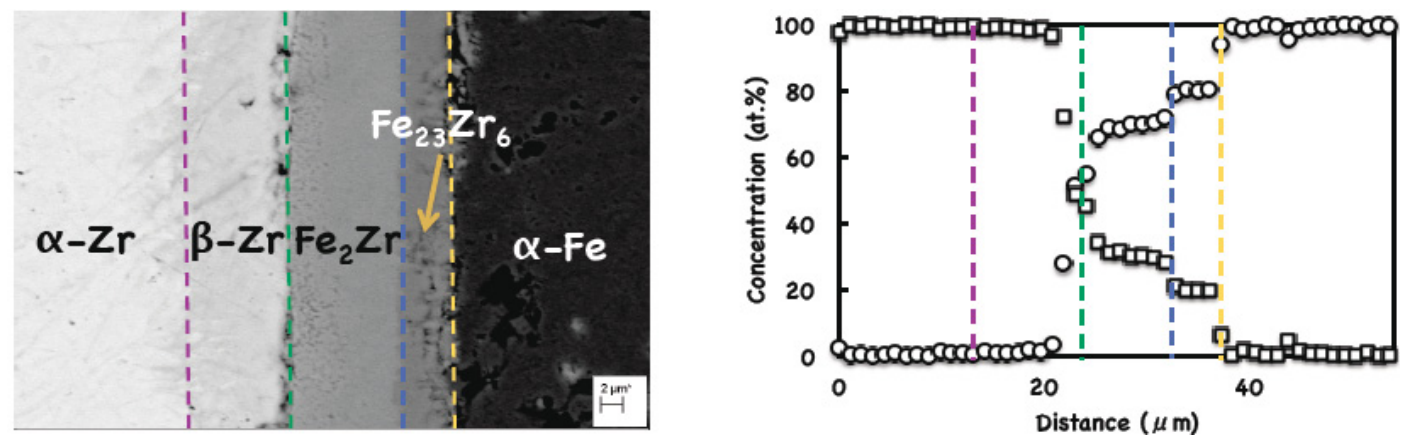

Figure 46. Backscatter electron micrograph of Zr vs. Fe diffusion couple annealed at $850^{\circ} \mathrm{C}$ for 15 days.

\section{Mo vs. Zr System}

Three diffusion couples between Mo and $\mathrm{Zr}$ were assembled and annealed at 750,850 , and $950^{\circ} \mathrm{C}$ for 30,15 , and 15 days, respectively. Backscatter micrograph and concentration profile of the resulting interdiffusion zone for the $950^{\circ} \mathrm{C}$ couple is shown in Figure 47 . Based on the profiles, there is a concentration gradient within the $\mathrm{Zr}$ solid solution in all three couples and no apparent $\mathrm{ZrMo}_{2}$ intermetallic phase. EPMA will be conducted on all three of these samples in order to obtain more accurate concentration profiles. The profiles will then be used to calculate the interdiffusion coefficient at each of the three temperatures.
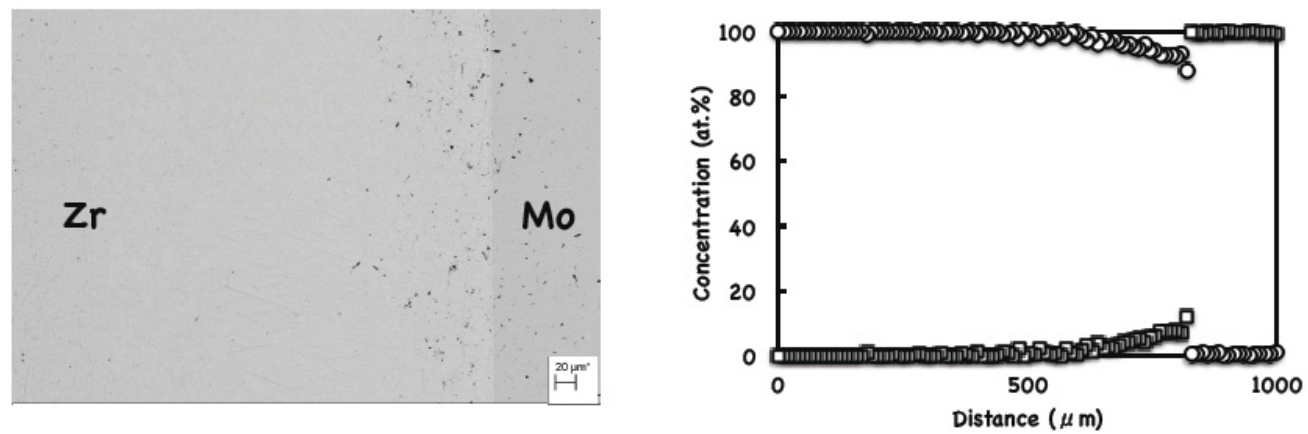

Figure 47. Backscatter electron micrograph and concentration profile of Mo vs. Zr diffusion couple annealed at $950^{\circ} \mathrm{C}$ for 15 days.

\section{U vs. Fe System}

$\mathrm{U}$ vs. Fe diffusion couples were annealed in the $\alpha$-U temperature range $\left(580,615,650^{\circ} \mathrm{C}\right)$ and the $\beta-\mathrm{U}$ temperature range $\left(680\right.$ and $\left.700^{\circ} \mathrm{C}\right)$. The BSE images and averaged concentration profiles of diffusion couples at $650^{\circ} \mathrm{C}$ are shown in Figure 48 . The 4 phases presented in Figure 48 are pure $\mathrm{U}, \mathrm{U}_{6} \mathrm{Fe}, \mathrm{UFe}_{2}$, and pure Fe from the left to the right side, which corresponds to the equilibrium phase diagram very well. The interfaces between each phase are uniform and planar. The $\mathrm{U}_{6} \mathrm{Fe}$ phase grew faster than $\mathrm{UFe}_{2}$. There is negligible solubility in both $\mathrm{U}_{6} \mathrm{Fe}$ and $\mathrm{UFe}_{2}$ phase, so the interdiffusion coefficient cannot be calculated. Instead, integrated interdiffusion coefficients, $\widetilde{D}^{\text {Int }}$ (Table 4), first kind growth constant ("extrinsic") $K_{I}$, and second kind growth constant ("intrinsic") $K_{I I}$ were calculated. Based on these data, we can conclude that diffusion in $\mathrm{U}_{6} \mathrm{Fe}$ is faster than in $\mathrm{UFe}_{2}$, and $\mathrm{U}_{6} \mathrm{Fe}$ grew faster than $\mathrm{UFe}_{2}$. The difference of activation energy for each phase between the $\alpha-U$ temperature range and the $\beta-U$ temperature range is small, which means allotropic transformation of $U$ played only a small role on the growth of $\mathrm{U}_{6} \mathrm{Fe}$ and $\mathrm{UFe}_{2}$. 

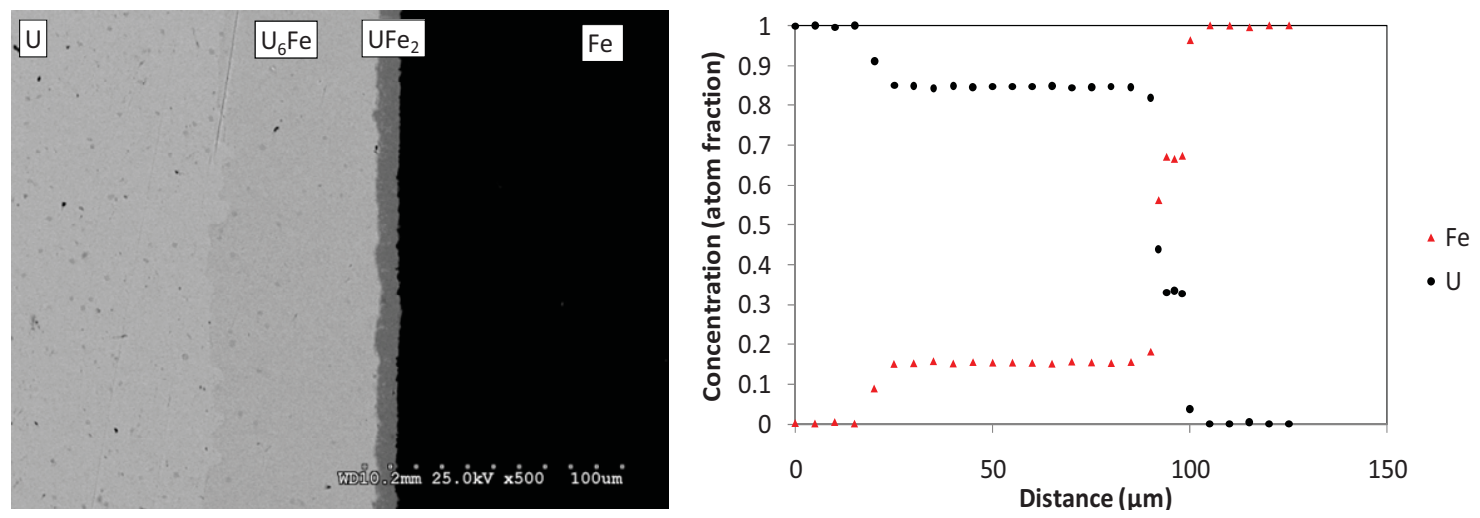

Figure 48. Backscatter electron micrograph and concentration profile of $U$ vs. Fe diffusion couple annealed at $650^{\circ} \mathrm{C}$ for 4 days.

Table 4. Integrated interdiffusion coefficients of $U_{6} F e$ and $U F e_{2}$ in $\alpha-U$ and $\beta-U$ terminal ends.

\begin{tabular}{|c|c|c|c|c|c|}
\hline U Phase & $\mathrm{T}(\mathrm{K})$ & $\begin{array}{c}\widetilde{D}^{I n t, U_{6} F e} \\
\left(\mathbf{x 1 0 ^ { - 1 6 }} \text { at. frac. } \mathbf{~ m}^{2} / \mathbf{s}\right)\end{array}$ & $\begin{array}{l}\mathrm{Q}^{\widetilde{D}^{m t, U_{\sigma}} \mathrm{Fe}} \\
(\mathrm{KJ} / \mathbf{m o l})\end{array}$ & $\begin{array}{c}\widetilde{D}^{I n t, U F e_{2}} \\
\left(\mathbf{x 1 0 ^ { - 1 6 }} \text { at. frac. } \mathrm{m}^{2} / \mathrm{s}\right)\end{array}$ & $\begin{array}{l}\mathrm{Q}^{\widetilde{D}^{I m t, U F e_{2}}} \\
(\mathrm{KJ} / \mathrm{mol})\end{array}$ \\
\hline \multirow{3}{*}{$\begin{array}{c}\alpha-\mathrm{U} \\
\text { (orthorhombic) }\end{array}$} & 853 & 1.56 & \multirow{3}{*}{148.46} & 0.040 & \multirow{3}{*}{243.37} \\
\hline & 888 & 3.60 & & 0.162 & \\
\hline & 923 & 7.63 & & 0.534 & \\
\hline \multirow{2}{*}{$\begin{array}{c}\beta-\mathrm{U} \\
\text { (tetragonal) }\end{array}$} & 953 & 11.35 & \multirow{2}{*}{112.73} & 0.680 & \multirow{2}{*}{225.97} \\
\hline & 973 & 15.21 & & 1.22 & \\
\hline
\end{tabular}

\section{U vs. Mo System}

$\mathrm{U}$ vs. Mo diffusion couples were assembled and annealed at $650,700,800,900$ and $1000^{\circ} \mathrm{C}$. The BSE images and concentration profiles of couples annealed at $1000^{\circ} \mathrm{C}$ is shown in Figure $49 . \mathrm{X}_{\mathrm{K}}$ and $\mathrm{X}_{\mathrm{I}}$ represent the position of the marker plane and the interface of $\gamma-\mathrm{U}$ and $\mathrm{Mo}$, respectively. At $650^{\circ} \mathrm{C}$, there are two phases of $U$ ( $\beta$ and $\gamma$ ) existing in the diffusion couple and the interface between the two phases is marked as $\mathrm{X}_{\beta \gamma}$. Interdiffusion coefficients, $\widetilde{D}$ in U-Mo alloys with mole fraction Mo, $N_{M o}$, varies from 0.02 to 0.26 in the temperature range from $650^{\circ} \mathrm{C}$ to $1000^{\circ} \mathrm{C}$ were calculated based on the curve fitted concentration profiles of Mo and are plotted in Figure 50.
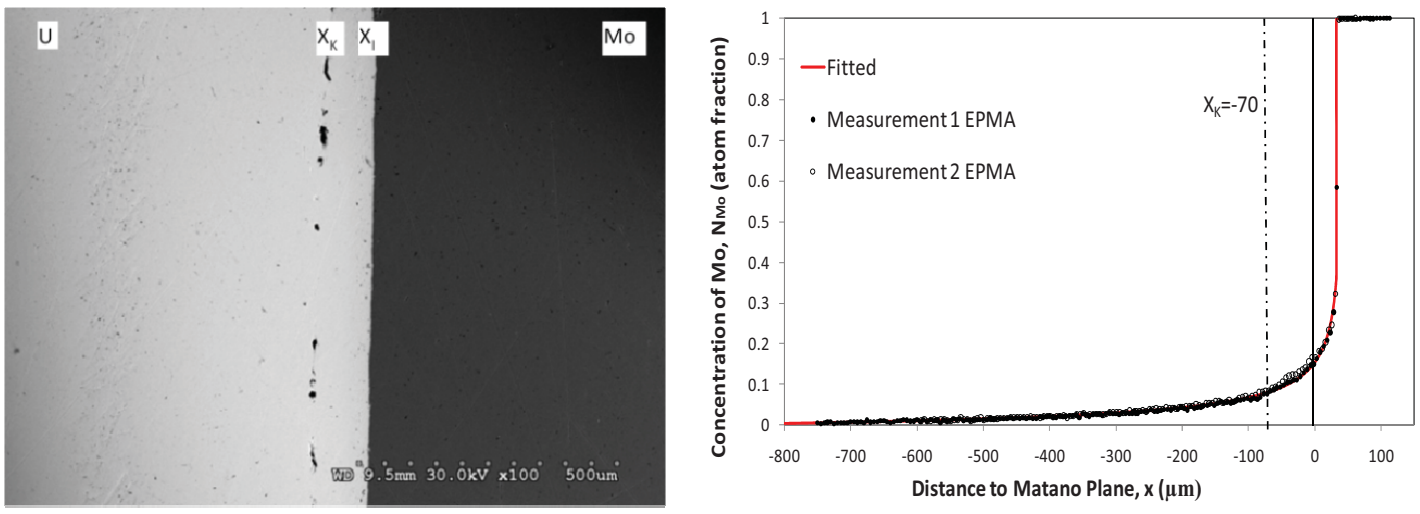

Figure 49. Backscatter electron micrograph and concentration profiles of $U$ vs. Mo diffusion couple annealed at $1000^{\circ} \mathrm{C}$ for 24 hours. 


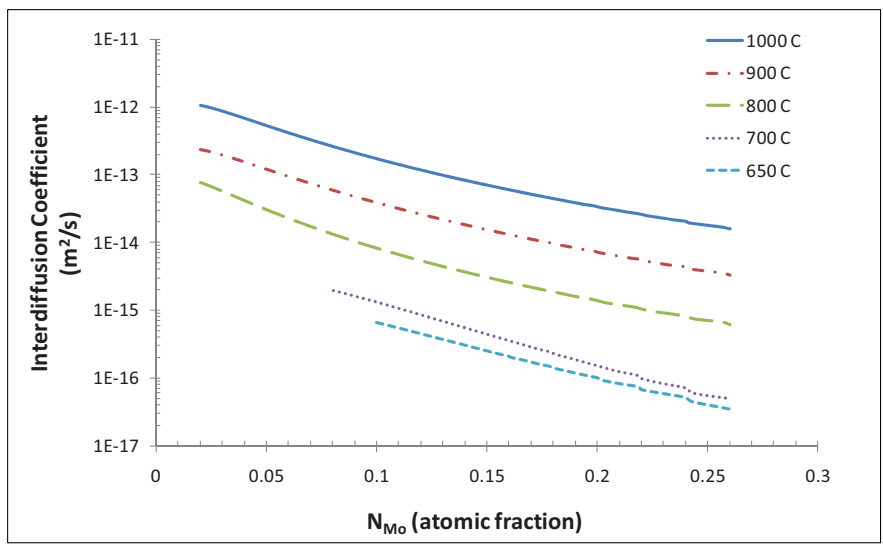

Figure 50. Interdiffusion coefficients of U-Mo alloy.

At each temperature, $\tilde{D}$ decreases with increasing Mo concentration. Figure 51 plots the temperature dependence of $\widetilde{D}$ at $N_{M o}=0.12,0.18$ and 0.24. $\widetilde{D}$, at a given composition, obeys the Arrhenius relation very well in the temperature range from 700 to $1000^{\circ} \mathrm{C}$. However, there is a small deviation in $\tilde{D}$ at the annealing temperature $650^{\circ} \mathrm{C}$. At the lowest annealing temperature $650^{\circ} \mathrm{C}$, grain boundary diffusion may play a more important role, which causes the interdiffusion coefficient to be higher than the Arrhenius relation. Another reason may be relatively large calculation error due to the short diffusion zone (about 30 $\mu \mathrm{m})$. The pre-exponential factor, $\widetilde{D}_{0}$, and activation energy, $Q$, of $\tilde{D}_{\tilde{D}}$ in the temperature range from $800^{\circ} \mathrm{C}$ to $1000^{\circ} \mathrm{C}$ at each $\mathrm{Mo}$ composition were determined.

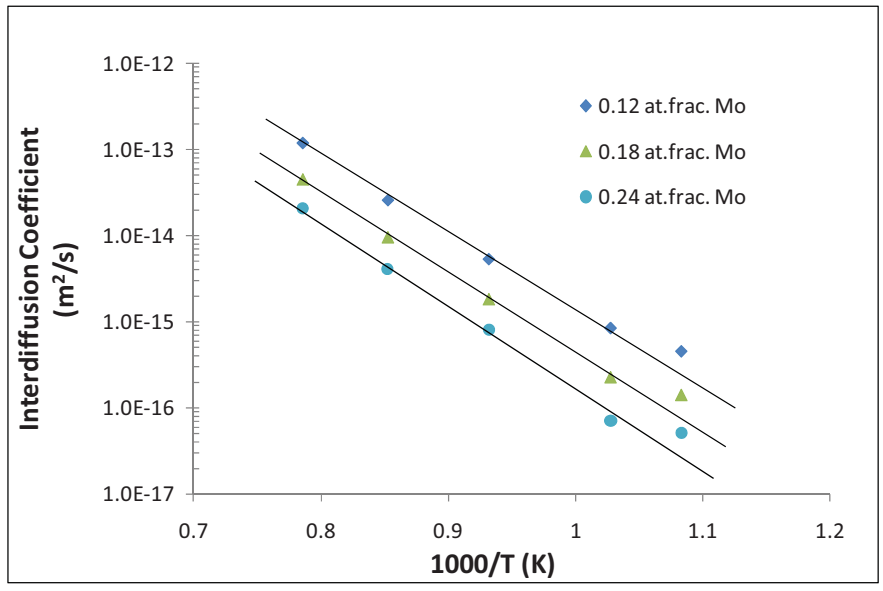

Figure 51. Temperature dependence of interdiffusion coefficients at NMo=0.12, 0.18, 0.24.

The intrinsic diffusion coefficients of $\mathrm{U}$ and $\mathrm{Mo}, D_{U}$ and $D_{M o}$ at the marker planes were computed. $\mathrm{U}$ intrinsically diffused about 5-10 times faster than Mo. The $D_{U}$ and $D_{M o}$ at the marker planes were applied to assess the tracer diffusivity, atomic mobility, and vacancy wind effect of $U$ and Mo in the $\gamma-U$ temperature range $\left(800,900,1000^{\circ} \mathrm{C}\right)$ when the activity or thermodynamic factor of U-Mo alloy at the marker composition is known.

\section{U-10Zr vs. Fe System}

Four diffusion couples between U-10wt\%.Zr and Fe were assembled and annealed at 630, 650, 680, and $700^{\circ} \mathrm{C}$ for 4 days. Typical BSE images of the interdiffusion zone are shown for the $700^{\circ} \mathrm{C}$ couple in Figure 52. Multiple layers with precipitate phases such as $\lambda$-phase, $\varepsilon$-phase, and $\chi$-phase were observed. The thickness of each layer was measured at each temperature and employed to calculate the parabolic 
growth constant and activation energies. The relation between growth constant of each layer and reciprocal absolute temperature are presented in Figure 53 where linear relationships are observed.

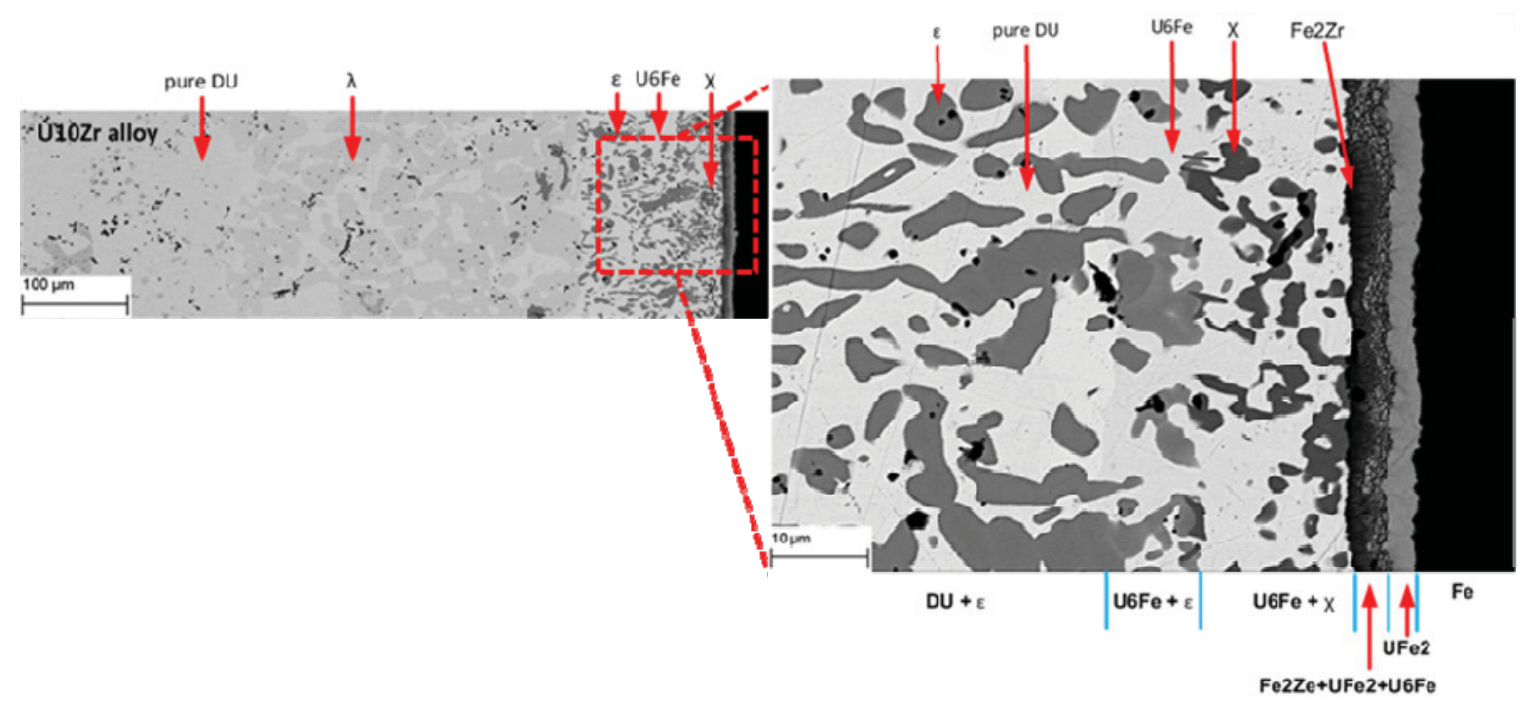

Figure 52. Backscatter electron micrograph of U-10wt.Zr vs. Fe diffusion couple annealed at $700^{\circ}$ for 4 days.

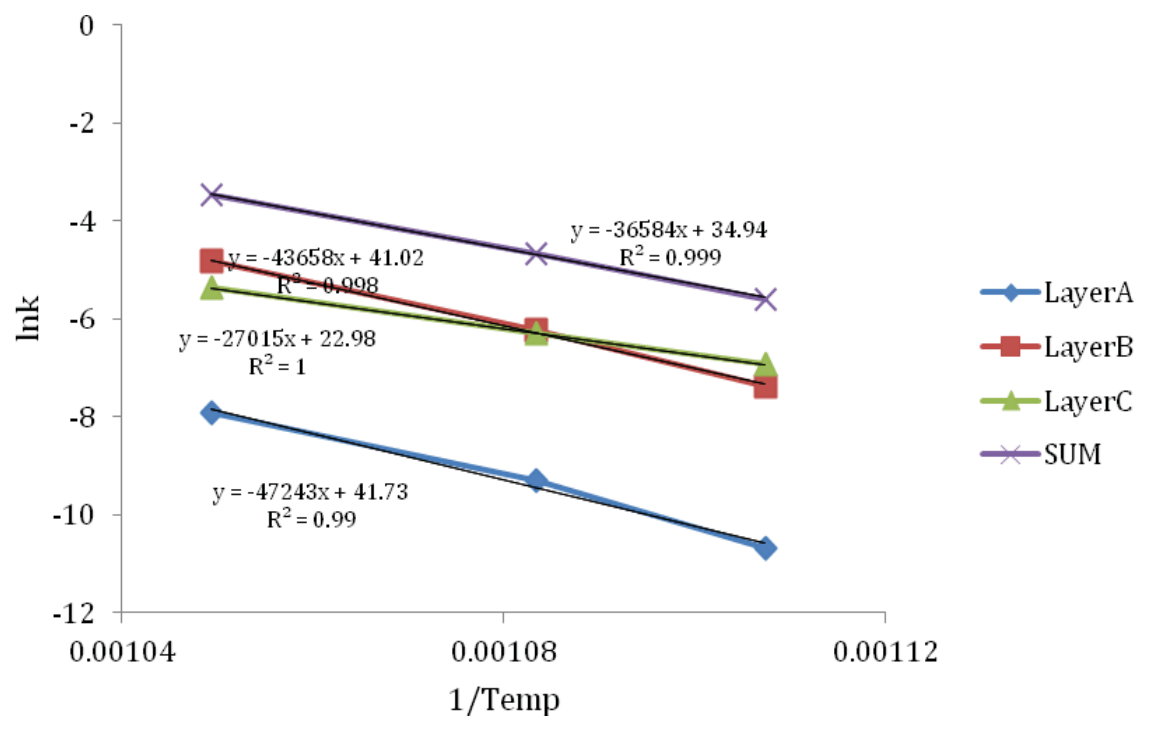

Figure 53. Arrhenius relation of growth constant for layer A, B, C, and SUM.

\subsection{Characterization Technique Development}

D. Hurley, david.hurley@inl.gov, INL

The work package is concerned with advancement of laser-based techniques to determine mechanical and thermal properties of nuclear fuel. A key accomplishment involving the initial construction stage of a multifunctional prototype instrument for laser ultrasound measurements is outlined below.

Two conceptual designs were developed: one based on the use of a free space interferometer and the other based on a fiber delivered interferometer. A market survey was conducted to identify a vendor that could meet or exceed the requirements for the measurement of laser ultrasound. Two types of 
interferometers were tested, the free space laser beam delivery and the optical fiber delivery, and it was determined that the free space interferometer was the only instrument that met the requirements.

A photograph of the prototype instrument after the initial construction stage is shown in Figure 54. The support column has been fabricated and mounted to the base plate. Mirrors and mirror mounts for guiding the detection beam up the column have been installed. The head support plates and mount brackets have been attached to the column. Structural components for the head have been procured and assembled. Detection beam scanning stages have been procured and mounted and the mirror mount assembly is partially complete.

Construction that remains to be completed includes: installation of the optics in the head, imaging system optics and camera installation, optics for generation beam, final detection beam mirror and lens, and incorporation of the interferometer.

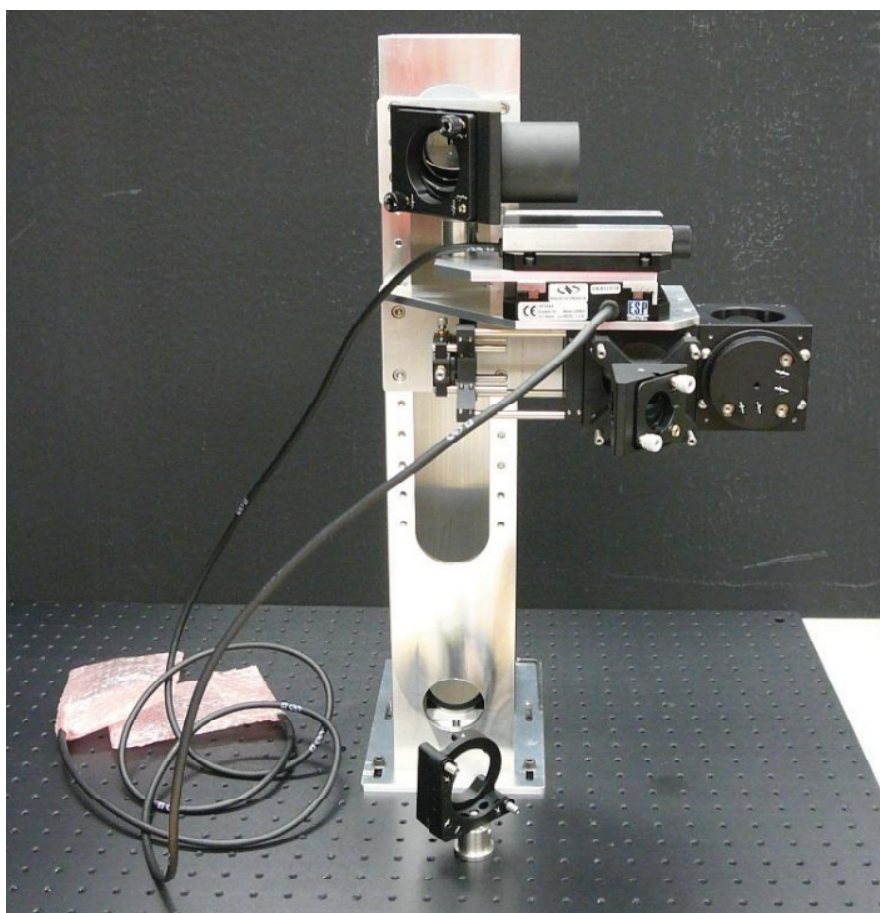

Figure 54. Photograph of prototype instrument for laser ultrasound measurements.

\subsection{Innovative Instrumentation}

\section{J. R. Kennedy, rory.kennedy@inl.gov, INL}

The FCRD program AFC Metallic Fuel Development technical area Innovative Instrumentation work package was tasked in FY 2011 to continue the development of state-of-the-art instrumentation for application with radioactive and highly radioactive materials. In addition, the pursuit of innovative concepts for in-pile measurement devices is reflected in the work scope of this work package. Significant progress was achieved in the development of the EPMA, $\mu$-XRD, and the FIB system as well as two concepts for in-pile measurements: a submersible thermoacoustic (TAC) sensor and a radio frequency/surface acoustic wave wireless transmission device. Highlights for each are described below.

\section{Electron Probe MicroAnalyzer}

Installation of the EPMA in the Analytical Laboratory was completed in FY 2011 and the basic performance of the instrument was tested including the secondary electron resolution, carbon decontamination, distance, and software. Quantitative element analysis at micrometer resolution was 
demonstrated on a silicon carbide ( $\mathrm{SiC}$ )-Pd diffusion couple. A set of actinide bearing standard materials (MOX, Pu, and Pu-Am alloy) was analyzed and found to be unsatisfactory due to inhomogeneities in composition.

\title{
Microfocus K-ray Diffractometer
}

The spatial and X-ray reflection resolution of the instrument was tested on a sample of $127 \mu \mathrm{m}$ diameter contacted wires of $\mathrm{Mo}, \mathrm{Nb}$, and $\mathrm{Ta}$ with a $25 \mu \mathrm{m}$ diameter beam. Line and surface mapping capability was demonstrated on a SiC-Pd diffusion couple in conjunction with EPMA studies to demonstrate continued development of complementary measurement techniques. Grazing incident type measurements were demonstrated on $\mathrm{CrSi} 2$ thin films with applications ranging from corrosion to interfacial interaction studies. Measurements on irradiated materials were demonstrated on ATR irradiated tubes of $\mathrm{SiC}$ and the results indicated that the material did not possess the expected $\alpha$-SiC structure.

\section{Focused Ion Beam}

The FIB tools located in the Electron Microscopy Laboratory (EML) and Center for Advanced Energy Studies (CAES) were used to develop methods for TEM and Atom-Probe Tomography (APT) sample preparation. Non-irradiated materials were used to develop the initial techniques, while TRISO particle fuel irradiated in the ATR showed proof-of-concept for TEM samples. In addition, the scripting capabilities of the FIB user interface have been used to prepare detailed structures.

\section{Thermoacoustic sensor}

A submersible TAC prototype sensor for in-pile wireless sensing was designed and tested. The TAC sensor has demonstrated temperature and axial displacement measurements as well as pressure independence to within 1\%. Proof of concept testing demonstrated the TAC sensor to have an axial displacement resolution of at least $100 \mu \mathrm{m}$. The prototype TAC sensing system is capable of producing an acoustic signal that can be heard throughout the laboratory while the device is entirely submerged in a water tank. The acoustic signal has ample signal-to-noise ratio for precise measurements from either a hydrophone submerged in the test tank or from a microphone in the laboratory. Temperature qualification of the TAC sensor shows that the resulting temperature invariance stayed constant to within $\pm 0.02 \%$ and the sensor has a temperature resolution of $0.05 \mathrm{~K}$. Pressure qualification indicates a measurable acoustic frequency deviation of $\pm 1 \%$ with a pressure change of $400 \mathrm{kPa}$ ( 4 atmospheres) equivalent to 44 $\mathrm{ppm} / \mathrm{kPa}$.

\section{Radio frequency/surface acoustic wave wireless transmission testing}

Proof of concept tests were performed using passive radio frequency identification (RFID) devices and through-the-water communication technologies. It is anticipated that these technologies can be engineered as accident tolerant monitoring systems in LWR cores by providing wireless high temperature monitoring operating in a passive mode. Successful preliminary testing was demonstrated for (1) wireless RF communications through water, (2) wireless RF communications in a reflective and scattering environment, (3) RF communication through a metallic test capsule, and (4) identification of sensor technology based on RF ceramic resonators. A device demonstrated communication of temperature data through 8 feet of fresh water, primarily limited by the experimental setup, at a near real-time data acquisition time of at least once per minute.

\subsection{Advanced Metallic Fuel for Fast Reactors - INL}

\author{
R. Mariani, robert.mariani@inl.gov, INL
}

INL is working with ANL on "Advanced Metallic Fuel Concept for Reliable Performance to Ultra-high Burnup," an outcome of the FY 2010 Innovative Transmutation Fuels Concepts Solicitation. 
This collaborative effort was one of three that were awarded funding as a result of that competitive solicitation.

The advanced metallic fuels concept incorporates major innovations that include decreased fuel smear density, annular compared to solid-rod fuels, coatings or liners on inner cladding wall, vented fuel pin, advanced fabrication methods, targeted fuel alloy additions, and U-Mo based fuel alloy while retaining compatibility with an electrochemical fuel recycle. A preliminary assessment and plan for concept development have been given.[1] The INL research effort is focusing on the latter two innovations, targeted fuel alloy additions and U-Mo based fuel alloys, to increase the chemical stability of metallic nuclear fuels. These innovations have the potential to enhance fuel reliability to high burnup.[2]

Fuel alloy additions offer the potential to bind lanthanide fission products as intermetallic compounds.[3] Lanthanide fission products are known to attack stainless steel cladding after they migrate to the periphery of the fuel slug and the fuel swells to contact the cladding. If, however, the lanthanide fission products can be chemically bound as intermetallic compounds, the lanthanides would be stabilized against attacking the cladding. Fuel alloy additions, or dopants such as palladium and indium, would be used in fresh fuel castings for this purpose.

U-Mo based fuel alloys are interesting because they have a low onset temperature for the body-centered cubic (bcc, or $\gamma$ ) phase.[4] Being cubic, the bcc phase expands isotropically with increasing temperature. Anisotropic fuel swelling was an early metallic fuel performance issue and was minimized through alloy additions that stabilized higher symmetry fuel phases.[5] U-Zr alloys were employed because of their higher solidus temperatures,[6] especially with the addition of plutonium for ternary $\mathrm{U}-\mathrm{Pu}-\mathrm{Zr}$ alloys.[7] However, the U-Zr alloys exhibit multiple phases at fast reactor temperatures, with the bcc phase being present only at the higher temperatures within a fuel element. Fuel swelling is not optimal compared to that for a single-phase bcc alloy, making U-Mo based fuels attractive, despite the lower melting temperatures for U-Mo alloys (and especially U-Pu-Mo alloys).

The INL research effort includes two collaborations with universities. Researchers at UNLV are pursuing efforts to understand the benefit of using palladium to bind lanthanides against attacking iron and stainless steel cladding. Researchers at the Colorado School of Mines (CSM) are investigating combinations of alloying metals such as zirconium and molybdenum to lower the onset of the bcc phase in uranium fuels while keeping the solidus temperature as high as possible. INL researchers conceived and developed the work scope and are guiding the university efforts. In addition, INL researchers are conducting experiments that are not suitable for the university facilities (e.g., experiments with plutonium).

Specific FY 2011 achievements include:

- Research plans for chemical stabilization of metallic fuels were fully developed as a result of literature survey and analysis, which included review of related post irradiation examination (PIE) data of metallic fuels.

- Possible transport mechanisms for atomic species in the fuel alloys were evaluated and the most likely mechanism was established.

- Plans were developed for U-Pu-Zr fuel experiments at the INL to incorporate palladium and other candidate dopants for binding lanthanide fission products as intermetallic compounds. These include out of pile separate effects tests and in pile tests. Experiment plans were also developed for more complex alloys that include molybdenum with plutonium.

- Experiments at INL continued to examine the use of palladium in fresh U-Zr fuels both with and without lanthanide metals present. 
- Out-of-pile, separate-effects experiments at UNLV were initiated for assessing the benefit and for characterizing the use of palladium as an additive dopant for binding lanthanides as intermetallic compounds. The initial results from UNLV corroborated and extended the preliminary results obtained at INL. INL results showed that palladium collocates with lanthanides in fresh fuel castings (Figure 55). UNLV examined diffusion couples where PdCe and PdNd intermetallics in contact with iron indicated no interaction, in contrast to strong interactions shown for cerium and neodymium in contact with iron (Figure 56).

- Experiments at CSM were initiated that are directed at understanding and characterizing U-Zr-Mo and other alloys to stabilize the solidus to higher temperatures while driving down the onset temperature for the bcc phase (to get the desired isotropic fuel expansion).

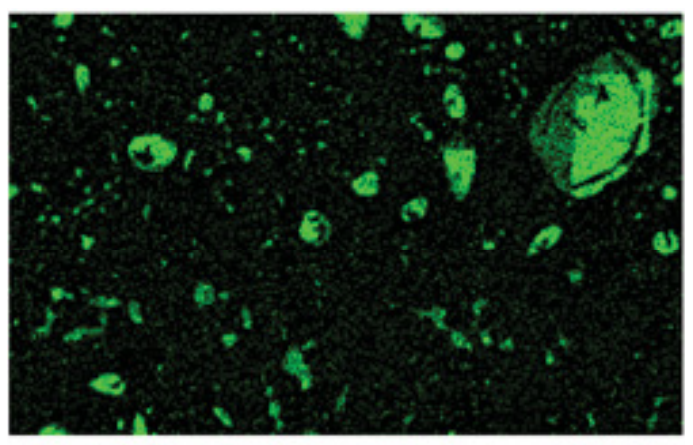

Pd La1

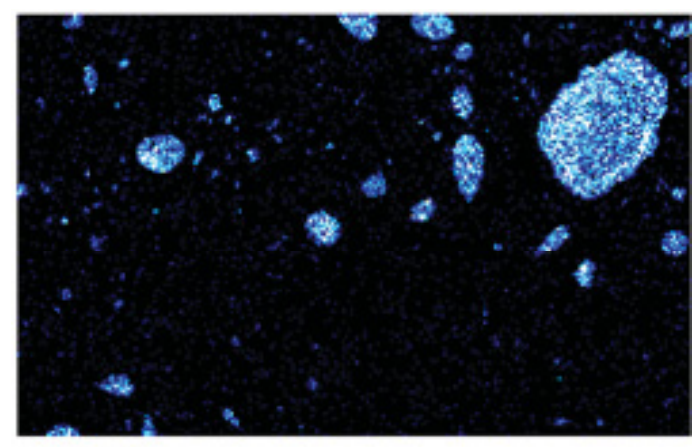

Nd La1

Figure 55. Dot maps for energy dispersive $X$-ray spectrometry of U-15Zr-1.6Pd-2.2Ln. (Ln = $53 \mathrm{Nd}-25 \mathrm{Ce}-16 \mathrm{Pr}-6 \mathrm{La}$, values in weight percent.) The palladium and total lanthanides are in the atomic ratio of 1-to-1, and the lanthanide content of the alloy represents approximately 8 atom percent burnup.

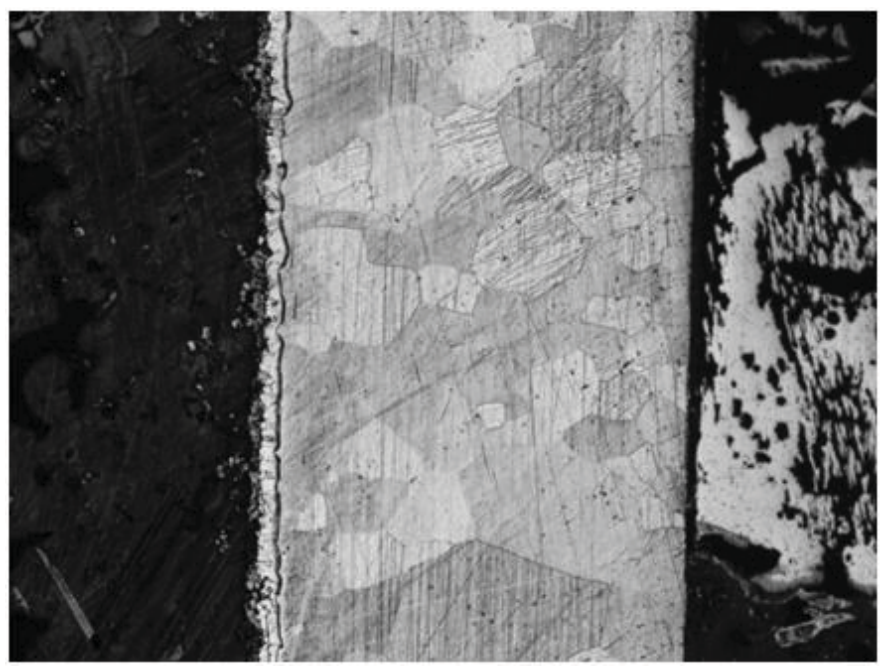

Figure 56. SEM of diffusion couple: $\mathrm{Nd} / \mathrm{Fe} / \mathrm{Pd}-\mathrm{Nd}$ alloy (materials, left to right) illustrating benefit of intermetallic formation between palladium and neodymium. The diffusion couple was heated at $700^{\circ} \mathrm{C}$ for 100 hours, and the double reaction zone is apparent on the left.

\section{References}

1. A. E. Wright, A. M. Yacout, T. K. Kim, Y. S. Kim, T. H. Bauer, W. D. Pointer, S. L. Hayes, J. I. Cole, and R. D. Mariani, "Ultra-high Burnup SFR Metallic Fuel: Preliminary Assessment and Plan for Concept Development, ANL-FCRD-317, September 2010. 
2. R. D. Mariani, D. L. Porter, T. P. O'Holleran, S. L. Hayes, J. Rory Kennedy, “ Advanced Metallic Fuel Concept Status Report,” INL/LTD-10-20431, November 2010.

3. R. D. Mariani, "Dopants for High Burnup in Metallic Nuclear Fuels," U.S. Patent Application No. 20110194667, published August 11, 2011.

4. H. Okamoto, "Mo-U (Molybdenum-Uranium)," Binary Alloy Phase Diagrams, 2nd Ed., Ed. T. B. Massalski, Vol. 3 (1990) pp. 2682-2683; Diagram No. 901671, (C) ASM International.

5. G. L. Hofman, L. C. Walters, "Metallic Fast Reactor Fuels," Materials Science and Technology, Volume 10, Nuclear Materials, Part I, R. W. Cahn, P. Haasen, and E. J. Kramer (Eds.), VCH, New York, 1994, pp. 1-44.

6. L. Leibowitz, R. A. Blomquist, A. D. Pelton, "Thermodynamics of the uranium-zirconium system," J. Nucl. Mater., 167 (1989) 76-81.

7. L. Leibowitz, E. Veleckis, R. A. Blomquist, and A. D. Pelton," Solidus and liquidus temperatures in the uranium-plutonium-zirconium system," J. Nucl. Mater., 154 (1988) pp. 145-153; L. Leibowitz, R. A. Blomquist, and A. D. Pelton, "Thermodynamic modeling of the phase equilibria of the plutonium-uranium system," J. Nucl. Mater., 184 (1991) pp. 59-64.

\subsection{Advanced Metallic Fuel for Fast Reactors-ANL}

A.E. Wright, aewright@anl.gov, ANL

Contributing authors: T. K. Kim, tkkim@anl.gov, D. Yun, diyun@anl.gov, A. M. Yacout, yacout@anl.gov, Y. S. Kim, yskim@anl.gov, W. D. Pointer, dpointer@anl.gov, A. R. Kraus, T. H. Bauer, thbauer@anl.gov, and K. Grimm,kgrimm@anl.gov, ANL

The innovative ultra-high burnup metallic fuel concept for the sodium-cooled fast reactor (SFR) is being pursued jointly by ANL and INL in response to the two grand challenges of the FCRD AFC Program. Those grand challenges are to achieve (1) a major increase in fuel burnup and performance over the current technologies and (2) near-zero-loss fuel fabrication processes.

An overview of the innovative design concept was described in the report "Ultra-high Burnup SFR Metallic Fuel: Preliminary Assessment and Plan for Concept Development" (Report ANL-FCRD-317) submitted in September 2011. As stated therein, the advanced metal-fuel concept is based upon the following innovations: decreased fuel smeared density, a coating or liner at the cladding inner surface to protect the cladding from chemical attack by the fuel at high burnups, venting of the fission gas to the primary coolant to maintain low gas pressure inside the fuel pin, consideration of a U-Mo-based alloy fuel system for potential improved performance relative to the reference U-Zr-based alloy, targeted fuel alloy additions to reduce fuel-cladding chemical interaction, and an advanced fabrication method that includes consideration of annular fuel.

A progress report on the work performed by ANL during FY 2011 was submitted (ANL-FCT-329). Corresponding progress made in FY 2011 by INL in this joint ANL/INL activity was reported separately (INL/LTD-11-23022) in August 2011. The following is a summary of the Argonne accomplishments.

In FY 2011, the Argonne work focused on four principal areas:

(a) Generating a reactor-core design (and attendant fuel element parameters and environment) that will allow practical SFR operation to ultra-high fuel burnup

(b) Developing a modern analytic capability needed to describe the key processes occurring within the innovative concept fuel and to predict fuel performance to ultra-high burnup

(c) Developing an analytical and experimental basis for evaluating the effectiveness of fuel alloy additions in reducing the potential for cladding chemical attack by lanthanide fission-products 
(d) Evaluating fuel pin venting to coolant design options and operational considerations associated with achieving the targeted ultra-high fuel burnup levels.

Fuel modeling issues include the control of lanthanide fission products to minimize the potential of chemical attack of the cladding (FCCI); fuel swelling behavior over the burnup range including ultra-high (30-40\%) burnup, particularly in U-Mo fuel; thermo-mechanical behavior of fuel with decreased smear density, sodium logging, and annular geometry, including mechanical interaction with the cladding (FCMI); and the phase structure and chemistry of U-Mo-based fuel and their impacts on the transport of fuel constituents and fission products.

Fuel pin designs to be evaluated will need to be consistent with practical reactor core designs capable of achieving ultra-high fuel burnup. Thus, detailed core design is a key part of the concept development and will help specify the parametrics boundaries within which fuel designs will need to fit.

One aspect of the design that seems clear is that, without major improvements in cladding materials, limiting the cladding internal pressure by venting the fission gas will be necessary. Various issues pertaining to venting fission gas into the primary coolant during normal operation were recognized long ago, and the approach is now to evaluate those issues within present contexts.

\subsubsection{Ultra-High Burnup Reactor Core Concept}

\section{T. K. Kim, tkkim@anl.gov, ANL.}

To assess the impact of the ultra-high burnup fuel on the core performance characteristics, homogeneous and heterogeneous ultra-high burnup sodium-cooled fast reactor (UHBR) core concepts were developed aiming for discharge burnup of $30 \%$ or higher. The homogeneous UHBR core concept consists of driver fuels only, while the heterogeneous UHBR core concept consists of driver fuels and internal, axial, and radial blanket fuels. The designs incorporate vented to-coolant fuel pins by necessity in order to achieve ultra-high burnup, in view of the current limitations in cladding properties and irradiation performance. Detailed core and blanket parameters for the design concepts made, mass flows of heavy-metal isotopes were computed, and inherent core safety considerations were described (Figure 57). 

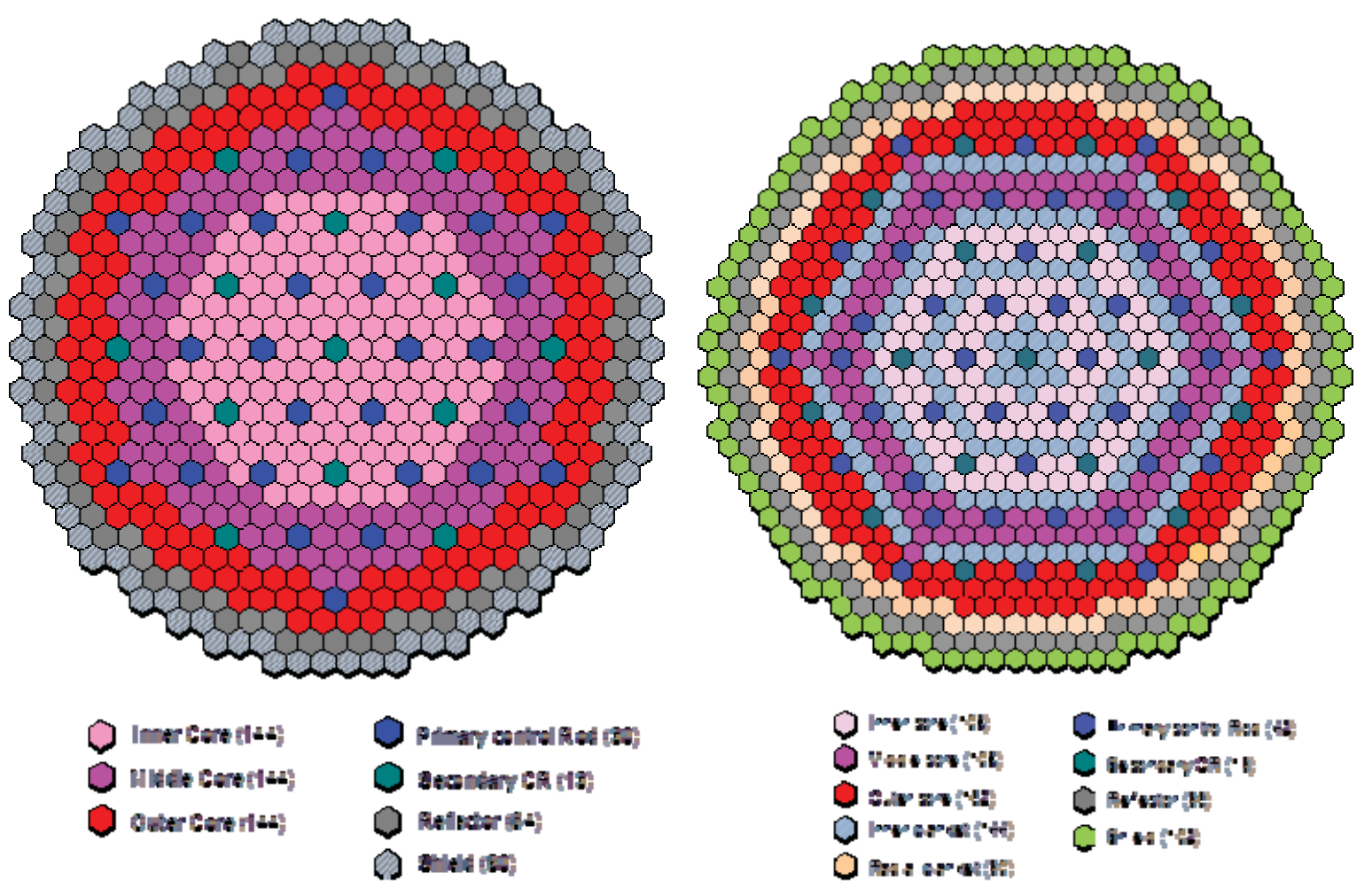

Figure 57. Homogeneous (left) and Heterogeneous (right) UHBR Core Concept

The UHBR core concepts adopted the innovative ultra-high burnup fuel (i.e., Mo-based metallic fuel, low smeared fuel density, fission gas vented, etc.). The design parameters and fuel management scheme of the core concepts were determined to maximize the average discharge burnup while simultaneously meeting critical safety criteria such as the fuel centerline temperature being below the fuel melting temperature and the cladding inner wall temperature being below the fuel-cladding eutectic temperature. It is noted that design criteria such as fast fluence limit of the cladding material and cumulative damage factor were not considered in the UHBR core concept development because it is assumed that current and future advances in fuel development and technology would lead to further extension of the discharge burnup or fuel residence time. Table 5 shows the primary design and core performance parameters obtained at an equilibrium cycle of the UHBRs.

The resulting burnup of the discharged driver fuel is $32 \%$ and $37 \%$ for the homogeneous and heterogeneous core concepts, respectively, with the fuel residence time in the core of about 20 years. The control of the power peaking factor and peak linear power density was a challenge in both UHBR core concepts because the large difference in burnup between the fresh and burnt fuels increases the power peaking factor. Thus, additional design efforts, such as fuel shuffling during the refueling interval, advanced driver fuel pins with absorbers, etc., are required to reduce the power peaking factor. The further reduction of fuel-smeared density to $50 \%$ was considered in this study, but the resulting core performance parameter changes were unfavorable regarding inherent safety features for unprotected accident scenarios. 
Table 5. Primary Design and Core Performance Parameters of Equilibrium UHBRs

\begin{tabular}{|c|c|c|}
\hline & Homogeneous UHBR & Heterogeneous UHBR \\
\hline Power rate, MWth/MWe & $3000 / 1200$ & $3500 / 1400$ \\
\hline Cycle length, month & 30 & 24 \\
\hline Number of batches (driver/inner blanket/radial blanket) & $8 /-/-$ & $9 / 12 / 15$ \\
\hline Fuel form (driver/blanket) & U-TRU-Mo/- & U-TRU-Mo/U-Mo \\
\hline Fuel length (active core/axial blanket), cm & $130.0 /-$ & $100.0 / 40.0$ \\
\hline Pin diameter (driver fuel/inner-radial blanket), $\mathrm{cm}$ & $0.94 /-$ & $0.87 / 1.00$ \\
\hline Fuel volume fraction (driver/blanket), $\%$ & $30.4 /-$ & $28.5 / 37.8$ \\
\hline Average TRU mass fraction of fresh driver fuel, $\%$ & 18.0 & 26.9 \\
\hline Fissile conversion ratio at equilibrium cycle & 0.71 & 0.93 \\
\hline Burnup reactivity loss, $\% \Delta \mathrm{k}$ & 5.6 & 3.1 \\
\hline Power sharing (active core/inner blanket/other), $\%$ & $100.0 /-/-$ & $60.3 / 26.2 / 13.5$ \\
\hline $\mathrm{HM} / \mathrm{TRU}$ inventory at BOEC, MT & $53.8 / 9.9$ & $82.7 / 10.8$ \\
\hline $\begin{array}{c}\text { Average discharge burnup, \% FIMA } \\
\text { Active core } \\
\text { Internal blanket } \\
\text { Axial blanket } \\
\text { Radial blanket }\end{array}$ & $\begin{array}{c}32.0 \\
- \\
- \\
-\end{array}$ & $\begin{array}{l}36.9 \\
28.9 \\
13.9 \\
10.3\end{array}$ \\
\hline Core average specific power density, MW/IHMMT & 55.8 & 42.3 \\
\hline Peak fast flux fluence (driver/blanket), $10^{23} / \mathrm{cm}^{2}$ & $15.6 /-$ & $14.5 / 19.3$ \\
\hline Linear power (average/peak), $\mathrm{kW} / \mathrm{m}$ & $23.1 / 52.0$ & $24.5 / 55.2$ \\
\hline Power Peaking Factor (BOEC/EOEC) & $2.26 / 1.94$ & $2.18 / 1.94$ \\
\hline Core average flux (driver/inner blanket), $10^{15} / \mathrm{cm}^{2}-\mathrm{sec}$ & $2.8 /-$ & $3.2 / 2.9$ \\
\hline Fast flux $(>0.1 \mathrm{MeV})$ fraction & 0.68 & 0.67 \\
\hline
\end{tabular}

\subsubsection{Thermo-Mechanical Modeling of Advanced Metallic Fuels}

D. Yun, diyun@anl.gov, and A. M. Yacout, ANL.

A thermo-mechanical model is being developed using the finite element (FE) multiphysics simulation code COMSOL and applying it to evaluate the performance of advanced metallic alloy fuels. This evaluation includes calculation of the temperature distribution within the modeled fuel element, fissiongas and fission-product induced fuel swelling, fuel-cladding-mechanical-interaction (FCMI), etc. Because this may be the first application of COMSOL to analyzing nuclear fuel performance, initial benchmarking was performed (for convenience, an oxide fuel simulation) with good results. Thermo-mechanical models were then built for $\mathrm{U}-\mathrm{Zr} / \mathrm{U}-\mathrm{Pu}-\mathrm{Zr}$ metallic fuel and time-dependent fuel swelling and creep models were 
built for metallic fuels U-10Mo, U-10Zr, and U-19Pu-10Zr in both solid and annular geometries. Fuel-cladding mechanical interaction modeling is continuing.

As one of the first steps for the metallic-fuel model validation, temperature distributions of cylindrical fuel element were simulated using this model and compared to the results of simulations reported in the literature. The comparisons showed good consistency between results from our model and from the literature.

Time dependent models have been developed that model fission-gas and solid-fission-product induced swelling and also thermal- and irradiation-induced creep (both in the fuel and the cladding) in both solid and annular metallic fuels including U-10Mo, U-10Zr and U-19Pu-10Zr. At the present time, available swelling correlations are applied to all the models. Attempt to incorporate a detailed swelling model into the COMSOL model met with difficulties because there were too many partial differential equations in the mechanistic model for COMSOL to handle. In the future, another route towards resolving the computational issue will be pursued: a mechanistic code will be used as a kernel of the simulation, with COMSOL providing critical information such as temperature profiles and hydrostatic stress distributions inside the fuel. In this way, the code will be able to handle the detailed power history, the contact between fuel and cladding or FCMI, etc.

Fuel-cladding contact mechanics modeling created problems of an unclear nature for swelling modeling in the case of 3-D solid fuel geometry. Several issues have been identified which may have contributed to the problem. 2-D axisymmetric models worked well in dealing with the contact mechanics issues with solid fuel geometry. Preliminary results of the 2-D axisymmetric models for U-19Pu-10Zr fuel showed that our model captured the shape of the axial distribution of cladding diametral strain and underestimated the magnitude when compared to available EBR-II PIE data.

The first priority in the next steps planned for the model development is to improve the swelling and creep models and further validate these models against available PIE data. Once these models are well established, models for constituent redistribution will be implemented in the code by constructing appropriate equations within COMSOL. A comprehensive validation effort will then need to be carried out to test the accuracy of the combination of models. When the validations are completed and the confidence of the models is established, detailed input corresponding to the current ATR irradiations can then be configured into the code to predict results from their PIEs.

\subsubsection{Atomistic Simulations of the Effect of Alloying Additions to U-Zr Fuel for Lanthanide Immobilization}

A.M. Yacout, yacout@anl.gov and G. Bozzolo, ANL.

Atomistic modeling of metallic fuels and computer simulations has studied the role of five different additives to $\mathrm{U}-10 \mathrm{wt} \% \mathrm{Zr}$ fuel, aimed at stopping surface migration of lanthanides ( $\mathrm{Ln}$ ). For the preferred cases ( $\mathrm{Sb}$ or $\mathrm{Pd}$ ), an amount of additive was recommended. All calculations were performed using the Bozzolo-Ferrante-Smith (BFS) method for alloys [1], a quantum approximate method that is best suited for economical but accurate calculations at the atomic level for multicomponent systems. The BFS method was chosen due to its ability to deal with an unrestricted number of elements without loss of accuracy, and its ability to provide a simple description of the role of each element in the resulting phase structure and surface phenomena.

The parameterization of the BFS method was performed based on in-house, first-principles calculations using the Linearized Augmented Plane Wave method and a database including fuel elements (U, $\mathrm{Zr}, \mathrm{Pu}, \mathrm{Mo}$, etc.), fission products (lanthanides, minor actinides, etc.), additives (In, Ga, Tl, Sb, Pd, $\mathrm{Sn}, \mathrm{Te}$ ), and cladding elements ( $\mathrm{Fe}, \mathrm{Cr}, \mathrm{C}, \mathrm{Ni}$, etc.). The calculations were performed in several stages: (1) examining the bulk behavior of additives to U-Zr and fission products, (2) examining the influence of 
surfaces, and (3) examining the energetics of individual atomic species to explain the formation of the observed phase structure and migration patterns.

The analysis of the results includes (1) the formation of precipitates in the bulk fuel among fuel elements, fission products, and alloying additions, (2) the surface segregation and migration patterns of all participating elements, and (3) the role of internal defects such as cracks, voids, and bubbles. Such behaviors were obtained from temperature-dependent Monte Carlo - Metropolis simulations using BFS for the energetics. The results were cataloged based on the ability of the different alloying additions (In, $\mathrm{Tl}, \mathrm{Ga}, \mathrm{Sb}, \mathrm{Pd}$ ) to achieve the desired effect of binding lanthanides to the bulk fuel and inhibiting their migration to the outer surface of the fuel, minimizing their interaction with cladding. Several features can be identified depending on the temperature dependence of the BFS total energy and its strain and chemical components, indicated in Figure 58. All U-Zr-Ln-X cases share basic features with the reference U-Zr-Ln case (Figure 58a), but with substantial differences in the location (with respect to temperature) of the most salient features. (Note that in Figure 58, the abscissa range is from 0-1400 K and in Figure 58b, $\mathrm{d}$, e, and $\mathrm{f}$, the chemical components become negative over part of the temperature range.). The characteristic temperatures of each case (defined below) are shown in Table 6.

- Minimum chemical energy $\left(\mathrm{T}=\mathrm{T}_{\mathrm{Ch}}\right)$ : maximum stability of $\mathrm{Zr}-\mathrm{Ln}$ and $\mathrm{Zr}$-Ln-X precipitates.

- $\quad$ Minimum strain energy $\left(T=\mathrm{T}_{\mathrm{St}}\right)$ : maximum segregation without surface reconstruction.

- Flattening of the chemical energy $\left(\mathrm{T}=\mathrm{T}_{\mathrm{F}}\right)$ : dissolution of the $\mathrm{Zr}-\mathrm{Ln}$ or $\mathrm{Zr}-\mathrm{Ln}-\mathrm{X}$ precipitates.

- High temperature increase of the strain energy, indicating surface reconstruction (terracing and faceting).

Based on simulations varying the amount of alloying additions, guidelines were established to determine the optimum amount of alloying additives needed to maximize the desired immobilizing effect, as a precursor for experimental verification. Defining $\mathrm{R}$ as the ratio of the concentration of additive $\mathrm{X}$ to the concentration of Ln atoms, the immobilizing effect of $X(X=S b, P d)$ seems to be optimized in the intermediate $\mathrm{R}$ range $(0.7<\mathrm{R}<1.4)$, and maximized (in terms of the temperature stability of $\mathrm{X}$-Ln precipitates) for the upper range of this interval $(1.2<\mathrm{R}<1.4)$. As a result, it appears that the case with $\mathrm{R}$ $=1.2$ (i.e., the amount of X approximately $20 \%$ higher than that of Ln present) is the best choice.

The mechanisms by which $\mathrm{Sb}$ and Pd achieve their final effect (similar in both cases) are somewhat different. Although both additives display similar behavior, Sb does so by binding Ln in Sb-Ln up to high temperatures, whereas Pd has sufficiently large surface energy to inhibit its migration to the surface through transport mechanisms. An equally weighted combination of both additives might be a viable option to maximize their independent behavior. 


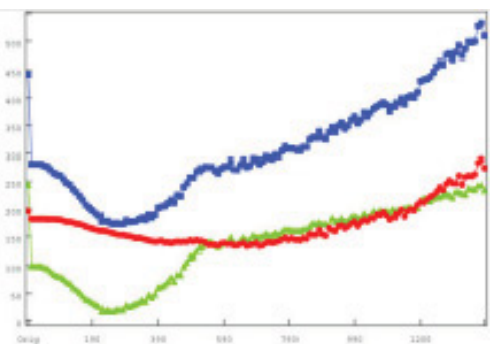

(a)

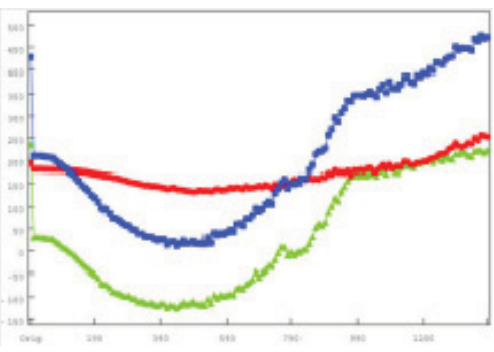

(d)

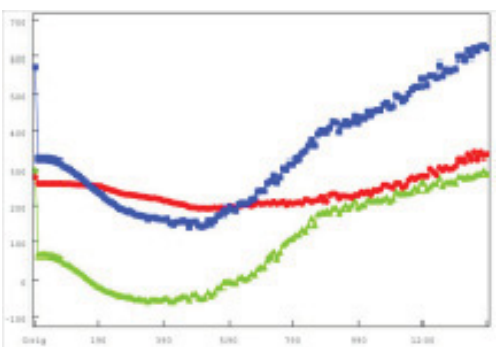

(b)

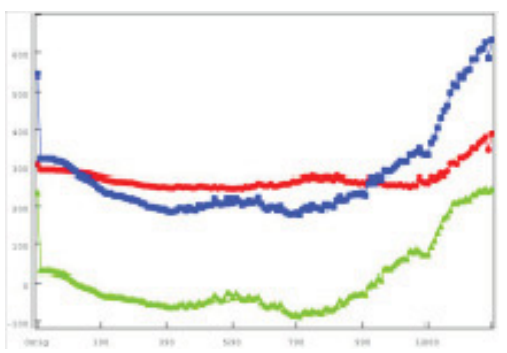

(e)

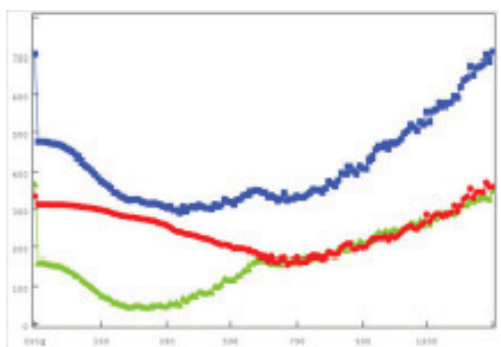

(c)

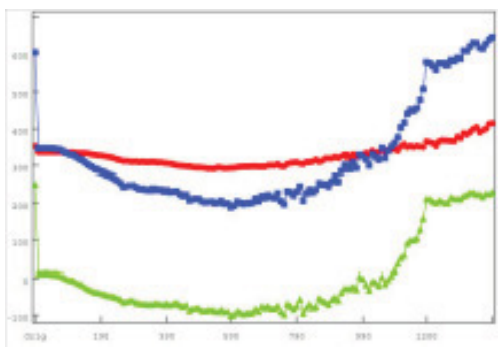

(f)

Figure 58. Total BFS energy (blue) of the computational cell as a function of temperature (0 to $1400 \mathrm{~K}$ ), and its strain (red) and chemical (green, lower curve) components for (a) the reference U-Zr-Ln cell (no additives) and the U-Zr-Ln cell with additions $X$ for $X=$ (b) In, (c) TI, (d) Ga, (e) Sb, and (f) Pd.

Table 6. Characteristic Temperatures (in K) of the Reference Case (U-Zr-Ln) and Different U-Zr-Ln-X Cases $(X=I n, T l, G a, S b, P d)$

\begin{tabular}{|c|c|c|c|c|c|}
\hline Case & \multicolumn{2}{|c|}{$\mathrm{T}_{\mathrm{St}}$} & \multicolumn{2}{|c|}{$\mathrm{T}_{\mathrm{Ch}}$} & $\mathrm{T}_{\mathrm{F}}$ \\
\hline U-Zr-Ln & \multicolumn{2}{|c|}{600} & \multicolumn{2}{|c|}{250} & 520 \\
\hline U-Zr-Ln-In & \multicolumn{2}{|c|}{530} & \multicolumn{2}{|c|}{550} & 850 \\
\hline U-Zr-Ln-Tl & \multicolumn{2}{|c|}{750} & \multicolumn{2}{|c|}{325} & 660 \\
\hline U-Zr-Ln-Ga & \multicolumn{2}{|c|}{480} & \multicolumn{2}{|c|}{400} & 980 \\
\hline U-Zr-Ln-Sb & 550 & 1150 & 400 & 800 & 1280 \\
\hline U-Zr-Ln-Pd & \multicolumn{2}{|c|}{550} & \multicolumn{2}{|c|}{600} & 1200 \\
\hline
\end{tabular}

\section{Reference}

1. G. Bozzolo, J. Ferrante, and J. R. Smith, Phys. Rev. 45 (1992) 493.

\subsubsection{Out-0f-Pile Experiment on Targeted Fuel Alloy Addition}

Y.S.Kim, yskim@anl.gov, ANL

An important aspect of the modeling will be that of describing the action of fuel alloy additions designed to diminish or eliminate chemical interaction between lanthanide fission products and the cladding. Both analytical and experimental approaches are being pursued to identify the optimum fuel additives and demonstrate their effectiveness. The analytical approach uses BFS methodology to compare the interactions between the fuel pin materials and various additives (palladium, antimony, gallium, indium, and thallium) particularly pertaining to the effectiveness of the additives in immobilizing the lanthanides within the fuel so as to prevent them from reaching the cladding. The experimental approach is investigating the interaction between fuel alloys and additives during fuel fabrication. 
Indium was selected as one of the candidates. An alloy of U-10Zr-2Ce-5In was fabricated and characterized. Figure 59 shows an optical micrograph of the sample cross section. In this image, it appears that the alloy has three phases: the continuous phase, precipitate phase, and the darker phase enclosed in the precipitate phase. Characterizations by SEM showed that there were indeed three phases that were vaguely observable in the optical image.

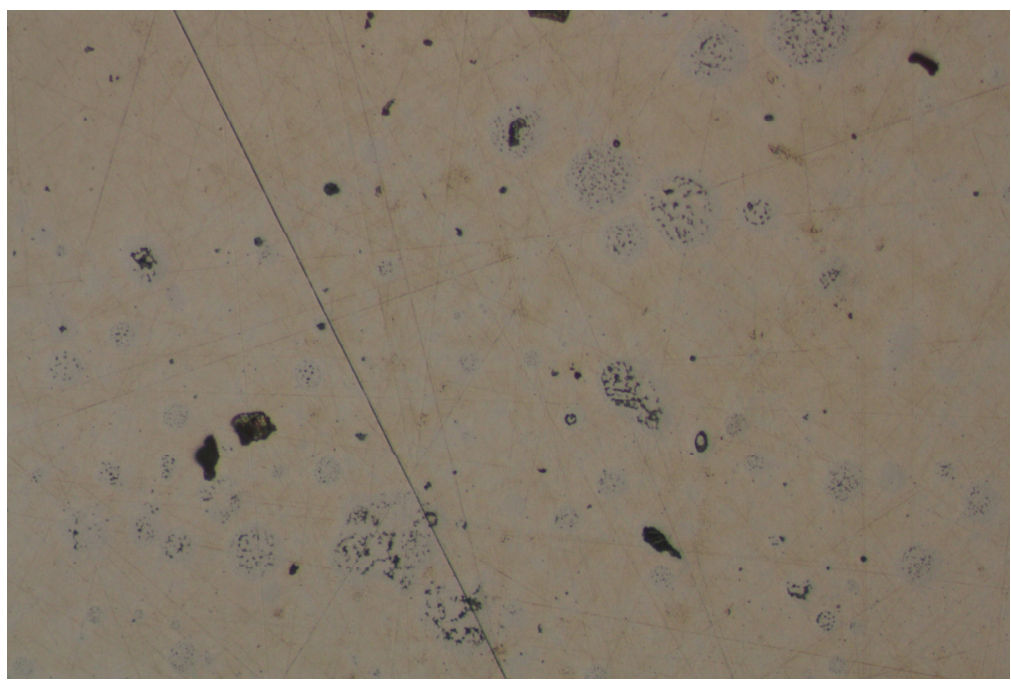

Figure 59. Optical micrograph of the cross section of U-10Zr-2Ce-5In alloy sample

Composition analysis (by energy dispersive spectroscopy) was performed, with analysis of three locations: one in the continuous phase, one in the precipitate phase, and one in the darker phase in the precipitate phase. The measured compositions at these three spots are given in Table 7, as determined from a complete fitting of the spectrum. Because Ce is found in the phase with $\mathrm{In}$, it appears that Ce forms a compound involved with In. The In to Ce ratio is about 2. Some indium found in the compounds with $\mathrm{Zr}$ is due to its affinity to $\mathrm{Zr}$ (even though indium tends to be preferentially attracted to Ce than to $\mathrm{Zr}$ ). However, in a real irradiation, this excessive amount of In will also be used to form compounds with Ce. This result confirms the effectiveness of its addition as an immobilizer for lanthanides. In addition, In does not appear to form high-In phases. The formation of such a high In compound (or even worse, In residing as a pure element) was a concern because of low melting of these that can cause liquid metal embrittlement of the cladding. When In forms a compound with fuel constituents, their melting points are much higher than the pure element.

Table 7. Composition analysis for phases observed (at\%)

\begin{tabular}{|c|c|c|c|c|} 
& In-L & Ce-L & Zr-L & U-M \\
\hline Spot \#1 & 3.6 & 0.1 & 7.0 & 89.3 \\
\hline Spot \#2 & 18.9 & 1.1 & 51.2 & 28.2 \\
\hline Spot \#3 & 27.2 & 15.7 & 39.2 & 17.9 \\
\hline
\end{tabular}

A similar test of $\mathrm{U}-10 \mathrm{Mo}-2 \mathrm{Ce}-5 \mathrm{In}$ is planned to be conducted next (including the same posttest analyses) for a direct comparison between U-Zr-based fuel and U-Mo-based fuel. Other additives of interest are being considered for subsequent tests. 


\title{
4.13.5 Vented Pin Sizing Analyses
}

\author{
W. D. Pointer, dpointer@anl.gov, A. R. Kraus, ANL.
}

In the evaluation of vented fuel concepts, two different design options can be considered:

- A fully open pin in which coolant sodium flows freely into and out of the pin

- A partially open pin in which fission gas can escape from the pin but coolant sodium does not enter the pin.

In the fully open case, the concentration of fission and activation products appearing in the coolant can be greatly increased as a consequence of dissolution of solid fission products into the sodium coolant. Therefore, the partially open concept is preferred. The analyses completed in FY 2011 provide the basis for a vented pin design using the diving bell concept, in which a drastically shortened fission gas plenum is connected to a vented diving bell volume by a long slender capillary. Among the various vented-to-coolant fuel-pin concepts, the diving-bell has historically been the preferred option because of its greater simplicity and likely higher reliability. To prevent coolant sodium contact with the fuel and to reduce the opportunities for capillary plugging, the diving bell vent plug must be designed so that coolant sodium does not enter the capillary under any normal operating condition.

The development of an optimized pin venting design requires the development and validation of a suitable analytical model of the concept as well as an analytical means to evaluate the radiological-driven ramifications of slowly releasing fission products from the fuel pin into the primary coolant during reactor operations. A simple engineering model has been used to evaluate the impact of geometric design parameters on the performance of the proposed diving bell vent design for a vented SFR fuel pin. Isothermal and non-isothermal changes in external pressure, as well as heat-up and cool-down scenarios, have been considered. The analysis suggests that the capillary tube and the diving bell volume should be as long as can be tolerated and that the fission gas plenum should be as short as can be reasonably achieved. Based on these initial scoping analyses, the nominal dimensions shown in Figure 60 and Table 8 are recommended as the baseline for future design studies.

The current analyses provide only supporting information needed to establish a nominal design for further analysis. The model does not account for frictional resistance or form losses in the vent openings or capillary itself. The model is also limited to steady-state snap-shots and does not provide a time line for the evolution of the sodium level or gas pressure in the pin.

Using the design guidance provided by these initial scoping studies, a more rigorous analysis of the vent performance and the time dependent evolution of the sodium coolant level is planned as future work. A computational fluid dynamics model of the vented pin is being developed using the commercial CFD software suite STAR-CCM+ to evaluate the time evolution of the sodium coolant level under different operating conditions to fine tune design parameters and optimize the proposed design. The model will 
correct the simplifications of the scoping analyses, including form and frictional losses, and will include the effects of the production of gaseous fission products.

Additionally, the small scale of the proposed vent plug enables the development of a small bench-top experiment that would provide a means to verify and validate the computational model used for design optimization. Two design concepts using different instrumentation strategies were proposed.

Table 8. Recommended Nominal Geometric Parameters.

\begin{tabular}{|c|c|c|c|}
\hline Parameter & & Unit & Value \\
\hline $\mathrm{D}_{\mathrm{po}}$ & Pin Outer Diameter & $\mathrm{m}$ & 0.008 \\
\hline $\mathrm{t}_{\mathrm{clad}}$ & Thickness of Cladding & $\mathrm{m}$ & 0.00052 \\
\hline $\mathrm{D}_{\mathrm{pi}}$ & Pin Inner Diameter & $\mathrm{m}$ & 0.00696 \\
\hline $\mathrm{D}_{\mathrm{co}}$ & Capillary Outer Diameter & $\mathrm{m}$ & 0.003 \\
\hline $\mathrm{D}_{\mathrm{ci}}$ & Capillary Inner Diameter & $\mathrm{m}$ & 0.002 \\
\hline $\mathrm{h}_{\mathrm{c}}$ & Length of Capillary & $\mathrm{m}$ & 0.1 \\
\hline $\mathrm{h}_{\mathrm{b}}$ & Length of Bell Volume & $\mathrm{m}$ & 0.1 \\
\hline $\mathrm{h}_{\mathrm{cb}}$ & Length of Capillary in Bell Volume & $\mathrm{m}$ & 0.08 \\
\hline $\mathrm{h}_{\mathrm{fp}}$ & Length of Fission Gas Plenum & $\mathrm{m}$ & 0.02 \\
\hline $\mathrm{t}_{\mathrm{plug}}$ & Thickness of plug & $\mathrm{m}$ & 0.02 \\
\hline
\end{tabular}

\subsubsection{A Radiological Perspective of Vented Fuel}

T. H. Bauer, thbauer@anl.gov and K. Grimm, kgrimm@anl.gov, ANL.

In assessing the radiological consequences of venting fission gases, and perhaps some volatile fission products, from fuel pins into the primary coolant, it is useful to provide a rough perspective on the potential radiological dose that might be involved. To do so, the relative "hazard" of radioactive components of fuel operated to ultra-high burnup (34 at.\% core-average burnup was assumed) is considered by scaling the material at risk for each isotope according to its associated relative biological hazard indicated (as an example) in DOE-STD-1027 for the hazard Category-3 threshold. Thus, the amount of each isotope "at risk" is divided by its Category-3 threshold and summed. A sum equal to unity, by definition of Category-3 values, corresponds to the amount of radioactive material which would produce less than $0.1 \mathrm{~Sv}(10 \mathrm{rem})$ doses at 30 meters based on 24 hour exposure. Results of this materialat-risk approach are presented in Figure 61 for several fission-product venting scenarios for 34 at $\%$ burnup fuel. The released-to-coolant fission product inventory, when venting only the noble-gas fission products into the coolant (as is intended for the vented fuel), was compared with more-extensive releases that would result under a broader range of scenarios ranging from operation of flow-through pin designs (in which the coolant flows within the cladding) to the hypothetical case in which all fission products and actinides are released into the coolant (either intentionally or by accident). On a per-mole-of-fuel basis, the results are as follows:

a) The radiological hazard from fission gases falls two orders of magnitude during the first week after release from the fuel and another order of magnitude during the following six months;

b) If the alkali metal fission products are also released, the hazard remains nearly constant at 15 times the initial fission-gas-only hazard level for the first six months before dropping appreciably; 
c) If all fission products are released, the initial hazard is about 500 times the initial fission-gasonly level and falls to twice the gases-plus-alkali-metals value at six months; and

d) If the actinides are also included, the corresponding hazard is initially only slightly higher than for the all-fission-product-release case but is still about 16 times the gases-plus-alkalimetals level after six months.

The time-dependent Category-3 sums computed using the preceding technique provide relative and absolute measures of radioactive hazard associated with vented fuels. These, in turn, can contribute to plant safety basis analyses along with associated requirements for future plant design and operation.

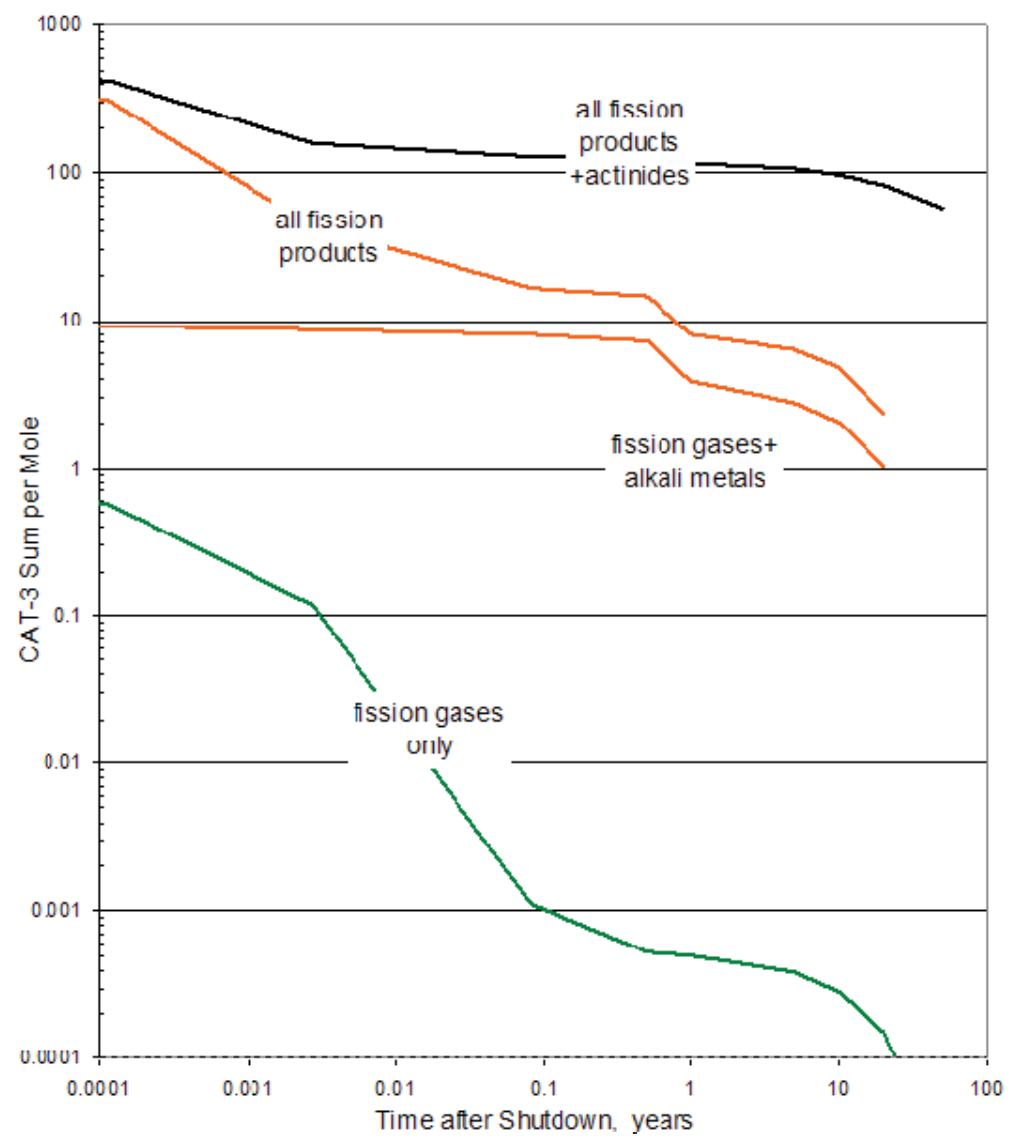

Figure 61. Relative measures of fission-product doses after fuel burnup to 34 at\%.

\subsection{Advanced Metallic Fuel for Light Water Reactor - PNNL}

R. Omberg, ron.omberg@pnl.gov, PNNL

Contributing authors: A. L. Doherty, K. J. Geelhood, C. H. Henager, C. A. Lavender, D.L. Love, and C. L. Painter

As part of its charter to develop and implement transformational changes, the FCRD Program solicited proposals for innovative transformational technologies in FY 2010. Of the 21 proposals submitted, three were chosen for further exploration and implementation. Among these was the development of Uranium-Molybdenum (U-Mo) metal fuel for use in commercial LWRs (LWRs). An earlier report (1) described a 3-year program leading to development and use of this fuel. A second report (2) contains a first principles feasibility assessment of this fuel. A multi-year plan (3) defining additional 
and more indepth experiments to further validate this fuel for commercial LWR use has been developed. A status report describing the work performed in FY 2011 was submitted (FCRD-FUEL-2011-000117).

The preliminary design of an experiment to establish the basic performance characteristics of the U-Mo alloy fuel, such as irradiation swelling of the U-Mo metal and the amount of fission gas released, was completed as were preliminary experiments on a fabrication process.

First estimates of heating rates in the LUMOS-1 proposed irradiation test were calculated and appear to be consistent with the expectations as discussed in the irradiation test plan. Linear heat rates range from $8-14 \mathrm{~kW} / \mathrm{ft}$ assuming the uranium enrichments on the order of $5 \%$. The rodlet's enrichment was varied and will be specified to obtain the linear heat rates $(6-10 \mathrm{kw} / \mathrm{ft})$ as identified in the test plan. The rodlets powers are not uniform and vary significantly in all dimensions. The overall rodlet axial and azimuthal variations are driven by the ATR thermal flux profile and to some extent, interaction with the other tests. The radial power variation in the rodlets is predominantly from self-shielding. Power peaking occurs near the ends of rodlets due to the exposed surface area on the ends of fuel pellet stack. This allows absorption of more neutrons and in turn creates a higher localized power density. As a result of these preliminary analyses, consideration is being given to using hafnium disks on the top and bottom of the fuel stacks to help flatten the local power rates. The LUMOS-1 experiment will take advantage of these rather large power fluctuations to obtain variations in burnup for a given time in core. Two rodlet stacks are designed to be the same distance from the core and will have nearly the same power distributions.

A FE code ANSYS was used to model and support the thermal analysis for the fuel rodlet and capsule design. Heat rates based on MCNP5 simulations discussed above were incorporated into the model. A continuous axial power curve was used for the axial profile. Three pellet models were developed - two annular pellets at different wall thickness and a solid pellet.

A linear elastic MATHCAD structural model was developed to assess the stresses in the U-Mo fuel, Zr-4 rodlet, and 304L SS capsule as a function of temperature, power, swelling and thermal expansion. It also allows the creep strain and internal pressure resulting from the release of fission gas as a function of burnup to be determined. A model for burnup as a function of linear heat rates was developed to support the preliminary modeling work. Burnup as a function of linear heat rate model for several irradiation periods was determined. These results yielded a plan to irradiate a set of fuel rodlets for about 200 days and allow another set to continue on for 400 days to obtain low burnup and high burnup data.

A detailed gas release model was created as a function of fission gas production rate which, in turn, is dependent on power level and irradiation time, $\mathrm{wt} \%$ of Mo in the alloy, temperature, dimensions, and number of pellets in a rodlet. The total moles of gas estimate also accounts for the helium gas included as backpressure during assembly process of the rodlet. The gas release model was incorporated into a structural model that has been created to evaluate the effects of internal pressure within a fuel rodlet.

The elastic modeling tools that were developed were used to analyze and bound the U-Mo swelling under nominal conditions to the higher power levels that might be encountered. These results suggest that at nominal conditions (i.e., $6.9 \mathrm{~kW} / \mathrm{ft}$ power level and $370^{\circ} \mathrm{C}$ ) the U-Mo annular fuel will not swell into the zircaloy cladding until after about 290 effective full power days in the reactor. However, at higher power levels, alternate dimensions, and irradiation temperatures this contact would occur prior to reaching 200 effective full power days. These results provide insight into how the capsules will need to be built and dimensions must be adjusted.

\section{Reference}

1. Painter, et al., "LWR U-Mo Fuel Development Plan," Pacific Northwest National Laboratory, PNNL-19722, (August 2010).

2. Gesh, et al., "LWR U-Mo Feasibility Study," Pacific Northwest National Laboratory, PNNL-19814, (August 2010). 
3. Painter, et al. "Engineering Design Plan-Uranium Alloy Fuel for LWRs," Pacific Northwest National Laboratory, S667146-EDP01 (April 2011). 


\section{GERAMIC-BASED FUELS TECHNOLOGIES}

Technical Lead: K. McClellan, kmcclellan@lanl.gov, LANL

Key challenges for AFC are the development of reliable, low-loss fuel fabrication methods and fuel technology development to enable major increases in fuel burn up and performance (reliability, power and safety) beyond current technologies. Ceramic fuel development accomplishments in the broad areas of processing, properties and performance for FY 2011 are discussed in this section. FY 2011 highlights include:

- Transitioned to a science-based approach for ceramic fuel development involving close coordination between theory, experiment and modeling efforts. A key aspect was completion of initial fuel development plans for $\mathrm{UO}_{2}$ (including $\mathrm{MOX}$ ) and $\mathrm{ThO}_{2}$-based fuels with associated irradiation test plans and separate effects test plans.

- Established co-conversion synthesis capabilities for reference oxide powder feedstocks, fabricated feedstock batches for separate effects, processing and properties studies.

- A flow sheet suitable for advanced oxide feedstock synthesis using the co-precipitation route was proposed as a reference case for future incorporation of this approach.

- A reference batch of thoria powder was procured and initial approval for processing and characterization of thoria-based fuels was established.

- Powder conditioning and processing studies were performed for reference urania powder and the reference, co-converted feedstocks. These studies improved pellet quality and reliability and provided material for subsequent oxide fuel structure/property relations studies.

- The ability to dynamically control oxygen to metal ration in urania was demonstrated and employed for processing and characterization studies.

- Master sintering curves were completed for reference urania powder enabling efficient fabrication of samples with specifically targeted densities.

- Initial microwave sintering studies were completed for urania demonstrating the ability to sinter $\mathrm{UO}_{2}$ with reduced sintering times.

- Interrupted sintering and dilatometry tests were performed on MOX fuel compositions under different atmospheres as part of a systematic study to establish the relations between processing conditions and resulting microstructure and chemistry for 80/20 and 70/30 MOX and two minor actinide-bearing analogues.

- Thermal transport studies were performed on various urania-based reference compositions providing fundamental data for development of improved thermal conductivity models. Insitu thermal diffusivity measurements as a function of time, temperature and atmosphere also provided insight into the role of phase and defect content during the early stages of pellet sintering.

- The initial set of urania samples were fabricated in preparation for the first ceramic rabbit test in HFIR.

- Technique development for synthesis of reference single crystals of urania and urania compounds was advanced for skull melting and via exaggerated grain growth.

- The technique for fabrication of urania thick films with embedded xenon was demonstrated yielding samples with nano-scale grain sizes analogous to high burn up structures in LWR fuels. Xenon contents were varied from a fraction of an atomic percent to over 6 at $\%$. 
- A systematic analysis of candidate fission gas getter concepts was performed and initial experiments demonstrated promising performance characteristics for both $\mathrm{Pd}$ and $\mathrm{Ni}$.

\section{FY 2011 Ceramic Fuel Approach}

Ceramic fuel development for FY 2011 focused on oxide (urania- and thoria-based) fuel and was a balance of effort seeking transformational R\&D while incrementally advancing fuel technology. This balance was established via fuel material processing and performance studies that advance fundamental understanding of the relationships for given fuel forms between fuel composition, pellet fabrication processing, corresponding material properties and subsequent fuel performance.

The ceramic fuel fabrication and characterization R\&D supports open, MOC, and closed fuel cycle options. The fundamental, science-based approach employed in this area relies upon close coordination of theoretical, experimental and modeling efforts and is substantially independent of spectrum (i.e., supports LWR and transmutation fuel development). The FY 2011 effort included fuel fabrication and performance studies employing separate effects-type testing but also still supports integral effect-type testing. Separate effects test studies are designed to establish and articulate the underlying physical processes at the lower length scales while supporting definition of phenomenology at the meso-scale and subsequent understanding of behavior at the engineering (continuum) scale. In addition to the resulting improvement of theoretical understanding of fuel fabrication and performance, the separate effects testing aspect of the program is intended to directly inform and support the development of predictive fabrication and performance models.

FY 2011 work was structured around the fact that the key issues for establishing low loss processing and improved fuel performance are materials issues. Therefore, a fundamental materials approach was taken to understanding the relationships between composition, processing, properties, and performance (see Figure 62) and then establishing enhanced ability to control processing and fuel performance. Research was primarily supported at Los Alamos National Laboratory (LANL) and ORNL, but critical collaborations were also supported at INL on irradiation testing and PIE under their technical areas. BNL lead the effort on the innovation concept for fission gas gettering. Highlights from the FY 2011 ceramic fuel development R\&D are categorized in this section.

\subsection{Processing of Uranium Oxide Powders Synthesized by Advanced Techniques}

E. Luther, eluther@lanl.gov, P. Dickerson, K. McClellan, LANL; S. Voit, ORNL

In FY 2011 LANL initiated processing studies on uranium oxide based powders produced by an advanced synthesis method, Modified Direct Denitration (MDD) performed at ORNL. Powders were also made at LANL by conventional methods for comparison. Powders made by MDD have the potential to reduce the amount of material lost during processing because fewer steps are needed to make a pellet. Each process step introduces loss during fabrication either due to scrap or simply material retained on equipment. These losses are acceptable for low value or low risk materials but become increasingly important as cost or health and environmental safety concerns increase. In addition, higher quality 
powders should result in more reproducible pellets which require less post processing (e.g., grinding) and which lower the probability of missing pellet surface flaws. These pellets should be more stable in pile, allowing safer operation by reducing fuel failure and allowing higher power and/or deeper burn.

In our initial studies, $(\mathrm{U}, \mathrm{Ce}) \mathrm{O}_{2}$ compounds were chosen for their applicability in a variety of fundamental studies. The advantage of the MDD process is that the components are completely dissolved and then co-converted to oxide. This approach allows intimate atomic mixing of the dissimilar species which is desirable to form a completely homogeneous powder. Further, this approach is fully compatible with feed streams produced by separations processes for used fuel recycling. In contrast, the conventional approach physically blends two dissimilar powders and then relies on solid state diffusion of the dissimilar atoms at elevated temperatures. Because the diffusion distances are quite large, and the driving force for diffusion decreases as the process progresses, this method is time consuming and energy intensive.

After synthesis, powders produced by MDD and conventional approaches were analyzed chemically using EDS, which allows the researcher to map the location of specific chemical species. Figure 63 shows the results of a uranium oxide powder with $30 \%$ cerium oxide made by the conventional method. Figure 64 shows a powder of the same composition made by MDD.

As seen in the figures, with the conventional approach mixing is incomplete as evidenced by the particle just below the center of the image in which the middle portion is cerium (yellow map) and the edges are uranium (blue map). In contrast, the powder produced by MDD is much more uniform at a finer scale. Although some segregation is observed, there is still a mixture of both species in all locations.

This study and others not described here have demonstrated some advantages of powders produced by the MDD process. In order to fully exploit the advantages of MDD powders, further studies are needed to explore powder flow properties for ceramic processing. Studies planned for FY 2012 are intended to produce powders that consolidate more uniformly and predictably than conventional powders.
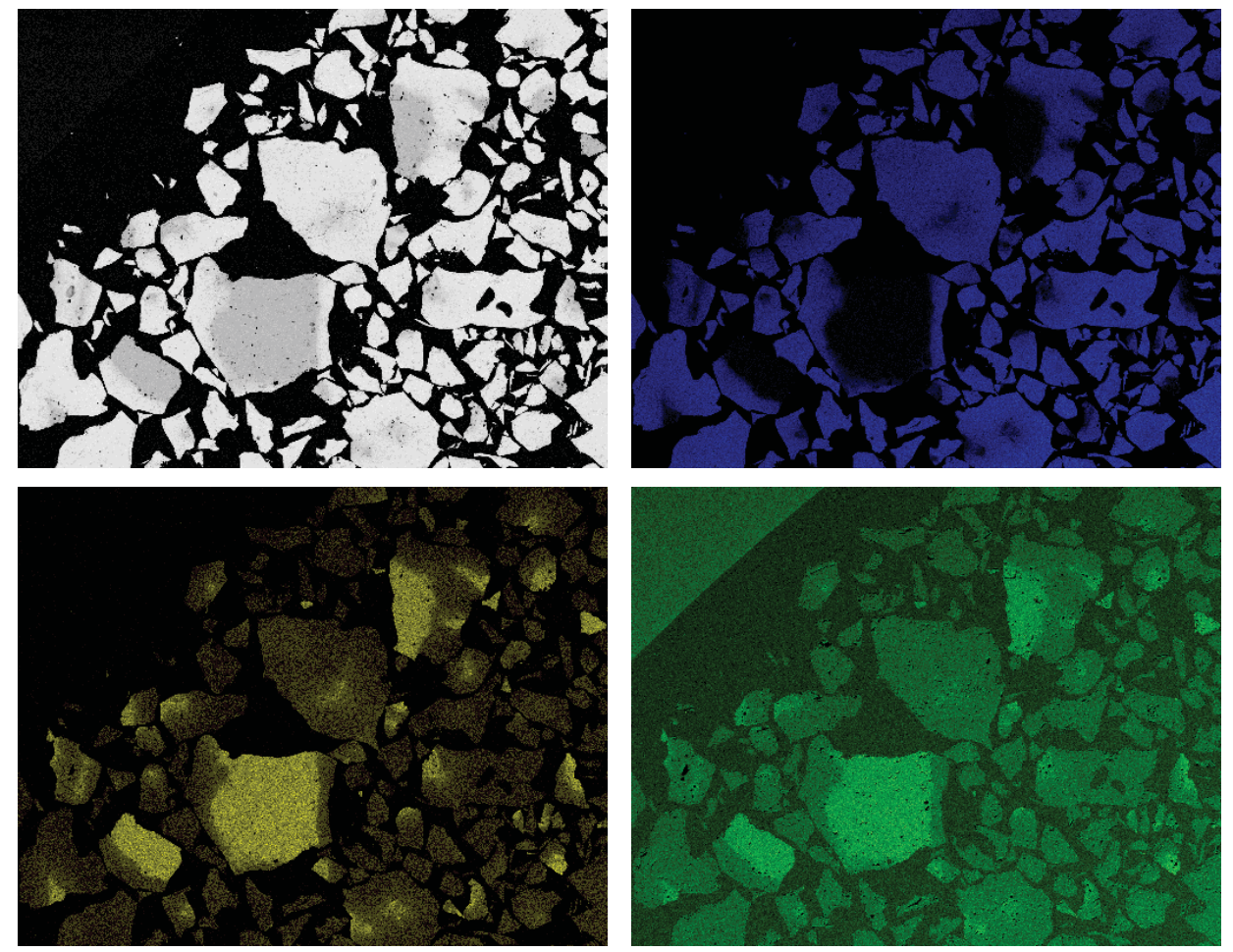

Figure 63. EDS analysis of DUO2 - 20 at\% Ce prepared by conventional processing after one heat treatment cycle: (A) Secondary Electron image, (B) U map, (C) Ce map, (D) O map. 

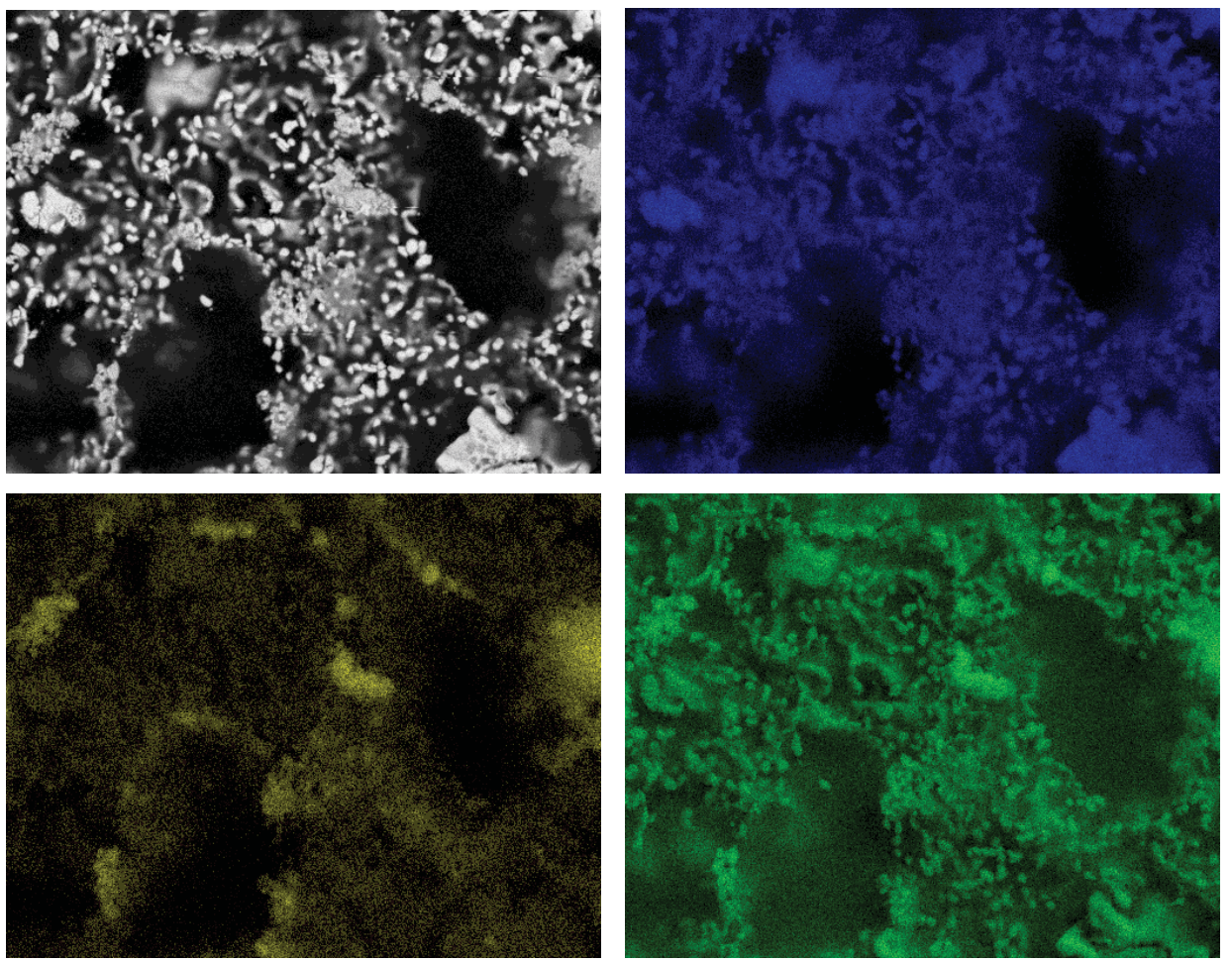

Figure 64. EDS analysis of fully reduced MDD UO2 - 20at\% Ce. (A) Secondary Electron image, (B) U map, (C) Ce map, (D) O map.

\subsection{Microstructural Control of Uranium Oxide Based Materials}

\section{E. Luther, eluther@lanl.gov, R. Forsyth, P. Papin, M. Emigh, K. McClellan, LANL; P. Peralta, ASU}

At LANL, research into microstructural control during pellet consolidation and sintering has enabled grain size control. The grain size of a ceramic fuel pellet is an important aspect due to the desire to contain fission products. Larger grained material is more likely to trap fission products within a single grain where diffusion is slower compared with diffusion along grain boundaries. Understanding the conditions under which pellets can be sintered to high density prior to extensive grain growth allows one to engineer the size of the grains that will be present in a fuel pellet in service. This work also supports modeling efforts which require data on materials that have highly controlled microstructures in order to separate the effects of numerous interrelated phenomena. By controlling the contribution of influencing factors such as grain boundaries, better data can be obtained as input for models allowing model refinements resulting in better fuel performance codes.

In Figure 65, pellets with densities of 92\% and 93\% density respectively are shown using Electron Backscatter Diffraction (EBSD) reveals grain size, shape, distribution and orientation with no additional sample preparation. Use of this technique on uranium oxide is a new approach developed at Arizona State University (ASU) and LANL for detailed study of pellet microstructures and generation of "real" microstructure for fuel model input. These two pellets were made under the same conditions with the exception of the sintering temperature. By sintering $300{ }^{\circ} \mathrm{C}$ hotter, the average grain size is increased from 3 microns to 21 microns. This effect is known in general for ceramics; however, the exact conditions enabling fabrication of pellets with starting high densities to allow this grain growth behavior in uranium oxide is an important new result. This data will be integrated into work on the MSC model (described in another highlight) in order to attempt to extend that model to predicting microstructural features. 
In preliminary experiments to create controlled porosity in fuel pellets, various starches with different average particle sizes were used as fugitive pore forming agents. Pores with controlled sizes can be produced by adding the appropriate starch to the ceramic material. Figure 66 and Figure 67 show electron microscope images of pellets with different pore distributions.
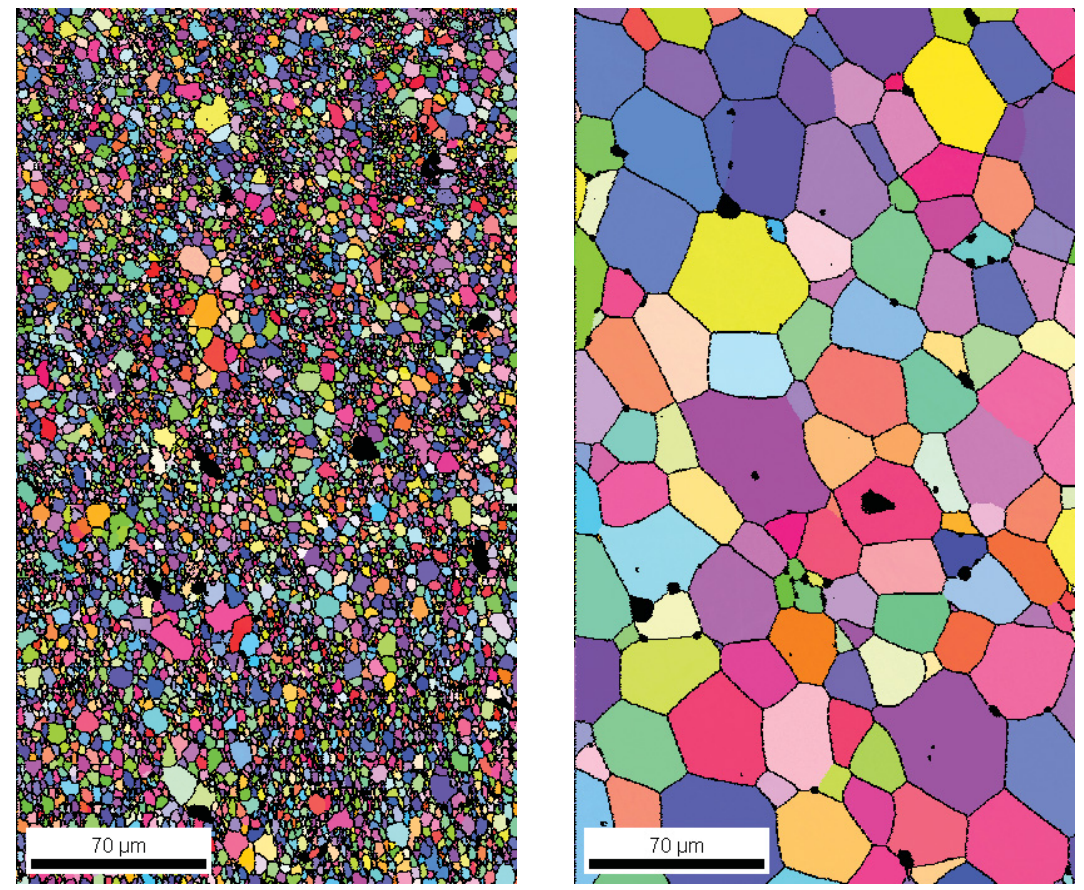

Figure 65. EBSD patterns of uranium oxide pellets sintered at $1350{ }^{\circ} \mathrm{C}$ (left) and $1650{ }^{\circ} \mathrm{C}$ (right). The pellets are approximately the same density but the grain size has been increased by a factor of 7 .

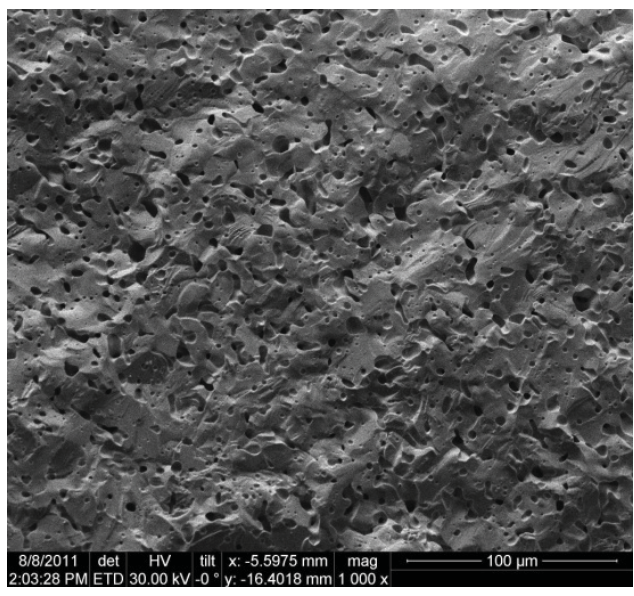

Figure 66. SEM image showing fine porosity induced by the addition of starch.

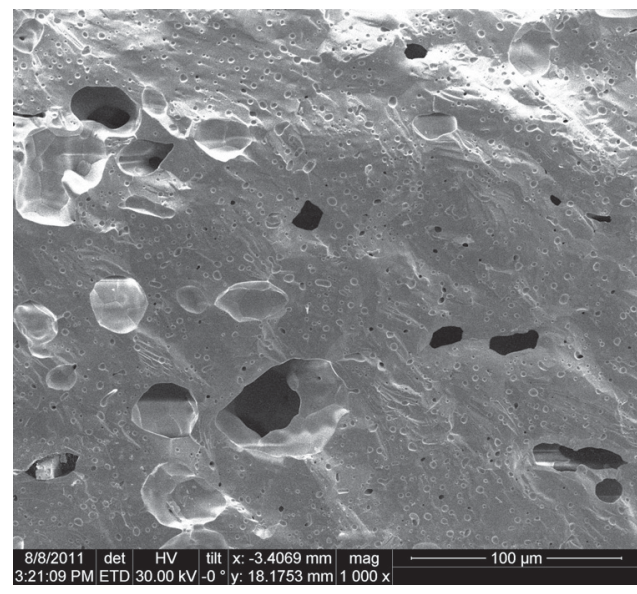

Figure 67. SEM image showing coarser porosity induced by the addition of starch.

With this research, we have established the initial capabilities for controlling grain size and controlled porosity. Work will continue in FY 2012 to achieve grain size control over a larger range of sizes and by alternative methods. Work on controlled porosity will also continue in order to demonstrate the formation of interconnected porosity. 


\title{
5.3 Oxide Thermochemical Modeling
}

\author{
S. Voit, voitsl@ornl.gov, T. Besmann, ORNL
}

Thermochemical model optimization of U-RE- $\mathrm{O}_{\mathrm{x}}$ systems is performed using a Compound Energy Formalism $(\mathrm{CEF})^{1}$ representation. A three sublattice model of the $\mathrm{UO}_{2 \pm \mathrm{x}}$ system has been developed by Gueneau et al. ${ }^{2}$ and this CEF framework is used as the basis for pseudobinary U-RE-O $\mathrm{O}_{\mathrm{x}}$ models because it accounts for hypo- and hyperstoichiometric charge compensating defects. Zinkevich et al. created a two sublattice CEF model for the $\mathrm{CeO}_{2-\mathrm{x}}$ system; ${ }^{3}$ however, the two sublattice model must be extended with a third sublattice before combining with the $\mathrm{UO}_{2 \pm \mathrm{x}}$ three sublattice model. The combined pseudobinary $(\mathrm{U}, \mathrm{Ce}) \mathrm{O}_{2 \pm \mathrm{x}}$ model will represent the complete fluorite fcc phase.

In FY 2011, the three sublattice model was extended to include excess oxygen at interstitial sites which will allow for modeling in the hyperstoichiometric regime. Figure 68 displays a comparison of the three sublattice $\mathrm{CeO} 2-\mathrm{x}$ model and the two sublattice model from Zinkevich against experimental data from the literature ${ }^{4,5}$ and the three sublattice model appears to display an improved fit. Figure 69 shows a plot of the experimental data points from Norris and Kay at $1123 \mathrm{~K}$. ${ }^{6}$ Also shown for comparison is the calculated fit to the data based on FY 2011 optimization of the improved the CEF model. The calculation displays very good fit to experimental data and extrapolation to lower $\mathrm{O} / \mathrm{M}$ values shows the correct trend.

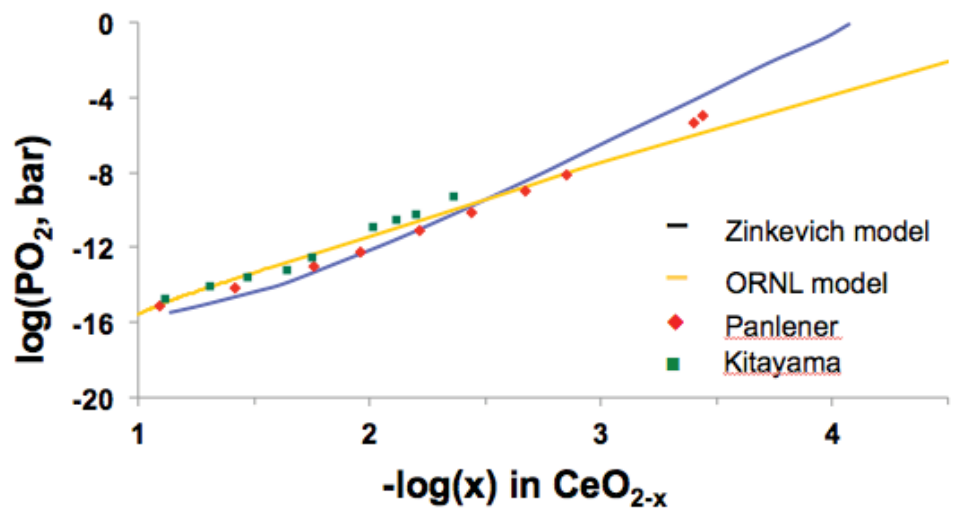

Figure 68. Comparison of ORNL and Zinkevich models to literature data.

Norris \& Kay: 1123K

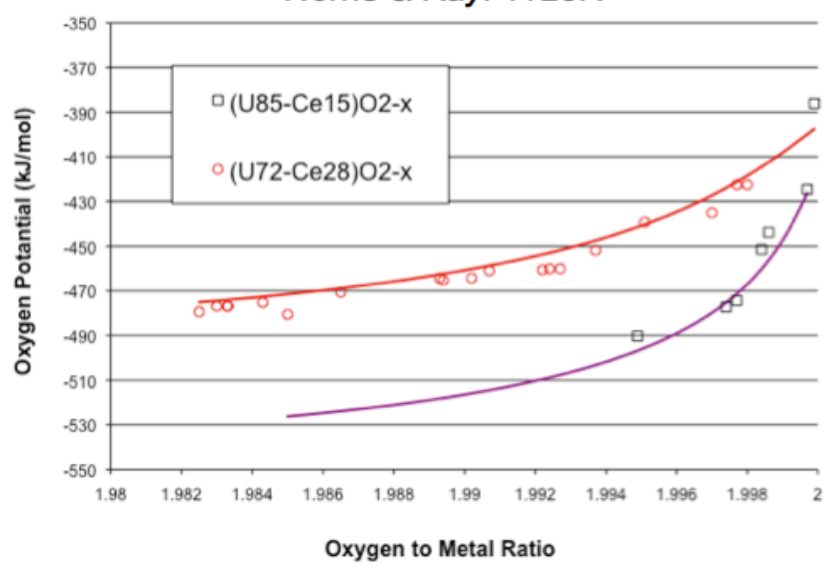

Figure 69. $\triangle G O 2$ versus O:M from experiment compared with CEF model for $(U, C e) O 2 \pm x$. 


\section{References}

1. Hillert, M., J. Alloys Compd. 320 (2001) 161-176

2. Gueneau, C., et al., J. Nucl. Mater. 304 (2002) 161

3. Zinkevich, M., et al., Solid State Ionics 177 (2006) 989-1001

4. Panlener, R., et al., J. Phys. Chem. Solids 36 (1975) 1213-1222

5. Kitayama, K., et al., J. Solid State Chem. 56 (1985) 1-11

6. Norris, D., Kay, P., J. Nucl. Mater. 116 (1983) 184-194

\subsection{Dynamic 0/M Control}

Darrin Byler, dbyler@lanl.gov, Erik Luther, Ken McClellan, LANL

The $\mathrm{O} / \mathrm{M}$ ratio plays a significant role in all aspects of a ceramic nuclear fuel from fabrication to performance. The $\mathrm{O} / \mathrm{M}$ has been shown to affect some of the key properties of the fuel including the thermal conductivity and melting point. Due to the range of $\mathrm{O} / \mathrm{M}$ ratios in ceramic nuclear fuels, work to understand the effects on processing, properties and the overall performance of the fuel have been ongoing. Early work done by Hagemark et al. on the $\mathrm{UO}_{2+\mathrm{x}}$ system revealed the broad range of oxygen partial pressures necessary to properly control $\mathrm{O} / \mathrm{M}$ with increasing temperatures. ${ }^{1}$ Efforts at LANL have been focused on gaining precise control over the $\mathrm{O} / \mathrm{M}$ ratio during sintering to enable evaluation of the effects on sintering kinetics, microstructure, physical and thermal properties.

The focus of FY 2011 work has been to refine the capability to precisely control the oxygen stoichiometry during the sintering process and evaluate the related effects on fuel properties. This work utilizes the dynamic gas mixing system developed in FY 2010 to control the oxygen partial pressure as a function of temperature to maintain a desired $\mathrm{O} / \mathrm{M}$ ratio within a furnace by precisely mixing two or more gases. Industrial fabrication of fuel pellets differs in that; the $\mathrm{O} / \mathrm{M}$ ratio is allowed to dynamically change during the sintering process, thereby changing the physical and thermal properties due to microstructural and stoichiometric variations. These changes do not allow for variations in feedstock or process parameters. To effectively produce high quality fuels with tailored characteristics, examination of the effects of the $\mathrm{O} / \mathrm{M}$ are essential for process optimization and control.

Fiscal year 2011 work has improved the capability to control the $\mathrm{O} / \mathrm{M}$ ratio of urania to \pm 0.01 , based largely upon oxygen partial pressure values calculated at ORNL. To illustrate the O/M control achieved, a plot of $\mathrm{O} / \mathrm{M}$ change with temperature is shown in figure 1a below. Figure $70 \mathrm{~b}$ illustrates an experiment to compare conventional to controlled $\mathrm{O} / \mathrm{M}$ sintering to evaluate sintering kinetics and understand the underlying mechanisms, which illustrates the effect of reduced oxygen availability early in the sintering experiment. This achievement in stoichiometry control has allowed experiments to be conducted to produce materials with tailored $\mathrm{O} / \mathrm{M}$ ratios for characterization of physical and thermal properties. This capability can be implemented for fundamental studies of fuel performance and optimization of fuel processing techniques. Additionally, this technique will be employed routinely to control $\mathrm{O} / \mathrm{M}$ ratios for studies involving thermochemical, thermophysical and melt point measurements. 


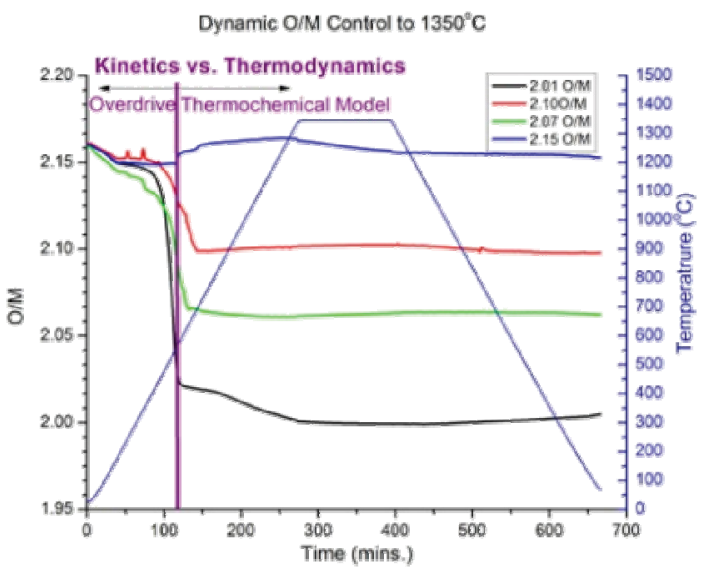

(a)

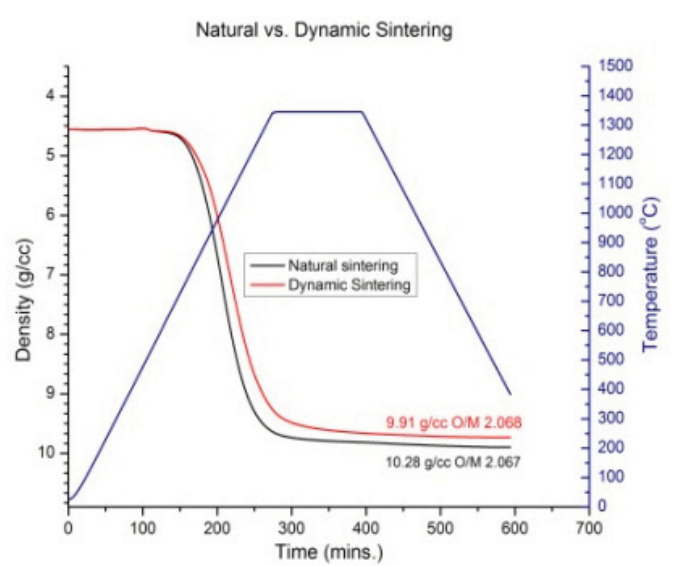

(b)

Figure 70. (a) TGA curves for the sintering of urania using a dynamic gas mixing system, (b) Dynamic vs. conventional sintering of urania resulting reduced density by controlled sintering.

\section{Reference}

1. Hagemark, K., Broli, M. Equilibrium oxygen partial pressures over the nonstoichiometric uranium oxides $\mathrm{UO} 2+\mathrm{x}$ and $\mathrm{U} 3 \mathrm{O} 8 \mathrm{-z}$ at higher temperatures, Journal of Inorganic Nuclear Chemistry. 1966, 28: pp 2837-2850.

\subsection{Sintering Modeling to Support Process Fabrication}

E. Luther, eluther@lanl.gov, B. Mihaila, P. Papin, K. McClellan, LANL

Uranium oxide fuel pellets are produced on an industrial scale by conventional ceramic processing routes, specifically, they are cold pressed and sintered. This conventional approach to the fabrication of fuel pellets for civilian nuclear reactors has been used for decades. The process is advantageous because it is inexpensive to manufacture large quantities of pellets quickly. Work at LANL seeks to improve the process in order to reduce the number of steps required to fabricate pellets to reduce process losses, allow remote handling, and result in more reproducible pellets which require less post processing (e.g., grinding). In addition, more reproducible pellets should be more stable in pile, allowing safer operation by reducing fuel cladding interactions and allowing higher power and/or deeper burn. In order to improve the pellet fabrication process, a fundamental, scientific understanding of powder processing science that integrates fabrication modeling to guide experimental progress is needed.

Fiscal year 2011 work has advanced an empirical model for sintering called the MSC. This year, an MSC was developed for uranium oxide such that its sintering behavior (density) can be predicted for any arbitrary time and temperature excursion. Also investigated was sintering behavior for non-stoichiometric uranium oxide. This data will ultimately be combined to form a Master Sintering Surface to allow sintering predictions for a wide range of starting conditions. This information sets the framework for continuing efforts that will extend this model to allow prediction of dimensional changes during sintering. Once completed, the model will allow prediction of sintering behavior in order to fabricate fuel pellets that are close to the final desired shape. Pellets that immediately achieve their final desired shape will require no additional processing (i.e., grinding), which is a step that introduces additional process losses. 


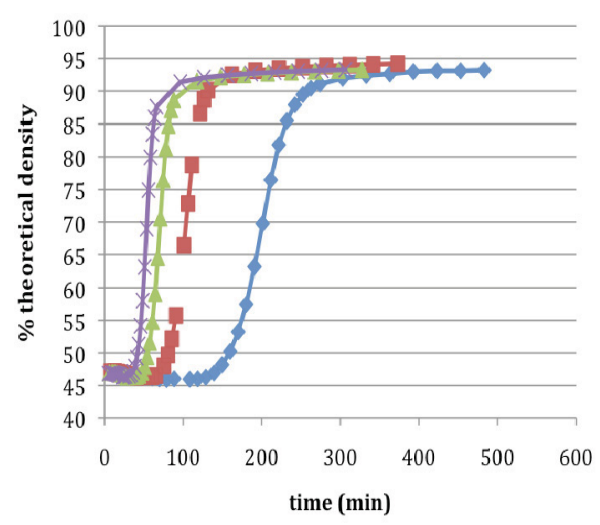

(a)

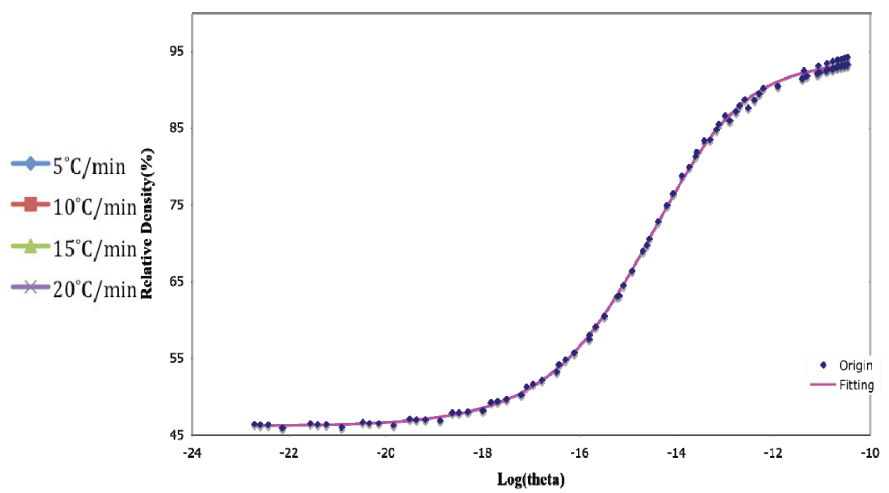

(b)

Figure 71 Empirical sintering curves (a) are collapsed in to a Master Sintering Curve (b) allowing prediction of the density over any arbitrary time/temperature excursion.

\subsection{Microstructural Characteristics of Sintered Urania}

\section{K. Rudman, K. Krishnan, H. Lim and P. Peralta, pperalta@asu.edu ASU}

The initial microstructure of oxide fuels can play a key role in their performance. The transport of fission products at low burnups may have a strong dependence on the grain size distribution, porosity and grain boundary (GB) characteristics (crystallography, geometry and topology), all of which, in turn, depend on processing conditions. These data are key to understand microstructural evolution of oxide fuels during processing and in-pile, and provide fundamental inputs for microstructurally aware models of fuel behavior at meso, micro and atomic scales.

Serial sectioning techniques were developed to obtain EBSD data for depleted $\mathrm{UO}_{2}$ pellets, manufactured under conditions that are representative of actual fuels. The EBSD maps were used to relate processing conditions to GB and porosity characteristics, with emphasis on special GBs according to the Coincident Site Lattice (CSL) model. Correlations between pore size and location in the microstructure were also studied. The information was also used to develop preliminary 3D FE models of mass transport to evaluate potential effects of GB networks on fission gas release.

The microstructure of $\mathrm{d}-\mathrm{UO}_{2}$ pellets with different densities ( $86 \%$ and $95 \%$ theoretical density [TD]) was characterized using EBSD scans. The CSL distribution of the insulator pellet (86\% TD) was compared with the denser pellet $(95 \% \mathrm{TD}$, as shown in Figure 72.

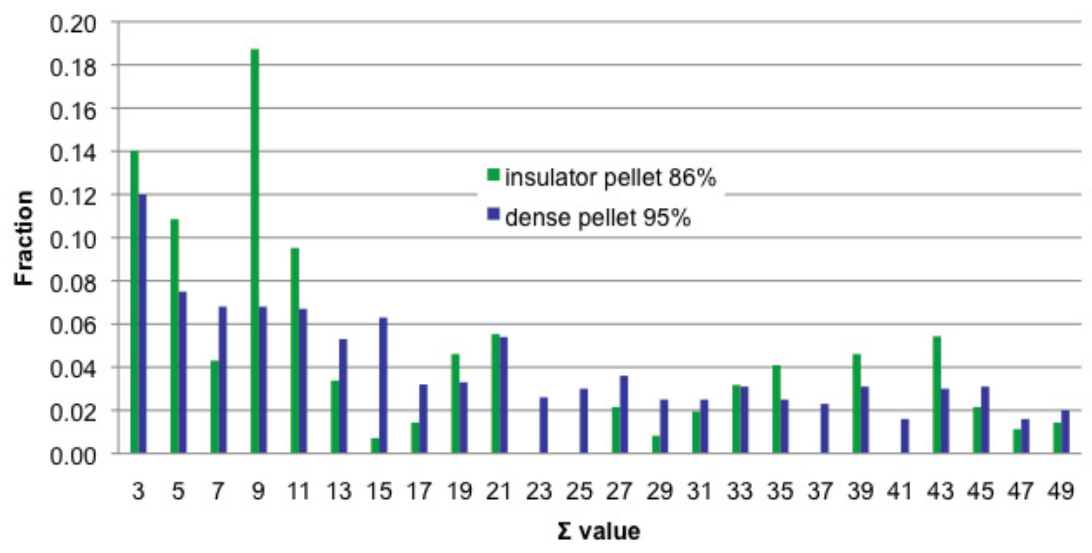

Figure 72. Grain boundary character distribution for CSL GBs for two pellet densities. 
Results showed a clear dependence of the CSL distribution on processing, while their total fraction remained approximately constant. The results also indicate that the denser microstructure had a more uniform distribution of special boundaries.

Correlations between porosity and microstructure were also observed, with larger pores located at triple points for all samples. Serial sectioning and EBSD maps were used to develop a 3D microstructure representation for the $95 \%$ TD pellet. This allowed the development of a 3D FE model of the GB network as well as measurements of triple point dihedral angles, which were found to follow a normal distribution, in agreement with theoretical predictions in the literature. In the model, the GBs are organized into 3 different groups - High, Low, and Bulk diffusivity GBs, based on their misorientation angles. The CSL GBs are assigned low diffusivity. Results indicate that mass flux localizes in GBs with higher diffusivity when the ratio of $\mathrm{D}_{\mathrm{HIGH}} / \mathrm{D}_{\mathrm{BULK}}$ exceeds $10^{4}$ and a clear percolation path arises for larger ratios (see Figure 73). Percolation behavior had a strong dependence on the geometry, location and misorientation of the GBs, which suggests that GB character could indeed play a key role on fission gas release at low burnups.

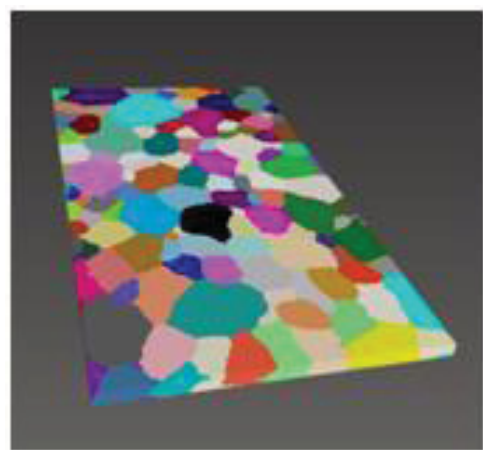

(a)

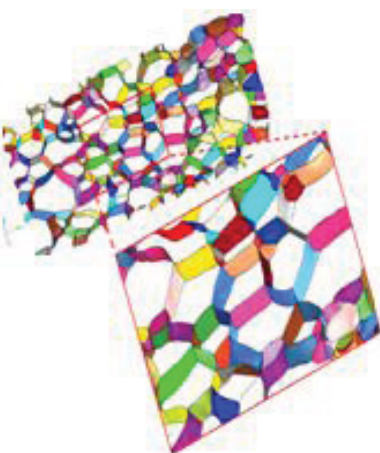

(b)

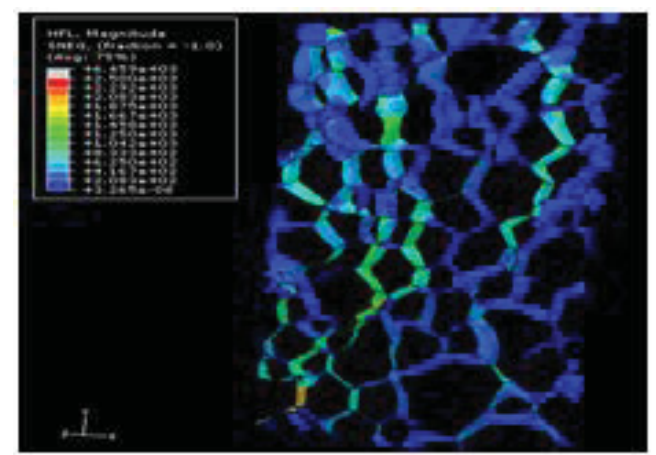

(c)

Figure 73. (a) 3D Reconstruction of the microstructure, (b) $3 D G B$ network and (c) $3 D G B$

\subsection{Sintering Kinetics of MOK and MA-MOX}

J. Mitchell, Jeremy@lanl.gov, S. Willson, LANL

Fiscal year 2011 work in the TA-55 Plutonium Facility focused on the sintering behavior of MOX and minor actinide-bearing MOX (MA-MOX). The objective of these studies is to improve processing knowledge so that higher quality and more reproducible pellets can be synthesized. Four pellet compositions were produced: $80 / 20\left(\% \mathrm{UO}_{2} / \% \mathrm{PuO}_{2}\right) \mathrm{MOX}, 70 / 30 \mathrm{MOX}, 80 / 20 \mathrm{MA}-\mathrm{MOX}$ (minor actinide content of $3 \% \mathrm{AmO}_{2}$ and $2 \% \mathrm{NpO}_{2}$ substituted in part for the $\mathrm{UO}_{2}$ in the $\mathrm{MOX}$ analogues), and 70/30 MA-MOX. Sintering behavior was then evaluated using interrupted sintering and dilatometry.

Pellets selected for interrupted sintering were measured for height, diameter, and mass. The furnace temperature was increased from room temperature to $400{ }^{\circ} \mathrm{C}$ in an $\mathrm{Ar}$ or $\mathrm{Ar} 6 \% \mathrm{H}_{2}$ atmosphere at 20 ${ }^{\circ} \mathrm{C} /$ minute. At $400^{\circ} \mathrm{C}$, the ramp rate was changed to $5{ }^{\circ} \mathrm{C} /$ minute, and the temperature was raised to the target temperature. Immediately following reaching the target temperature, the temperature was decreased at a rate of $200{ }^{\circ} \mathrm{C} /$ minute back to room temperature. The finished pellets were measured for diameter, height, and mass, and dimensional and density changes recorded. One pellet from each batch was submitted for $\mathrm{O} / \mathrm{M}$ analysis, $\mathrm{x}$-ray diffraction, ceramography, and electron microprobe analysis. An example of the interrupted sintering curves is shown in Figure 74.

Each dilatometry experiment was performed in UHP Ar or Ar- $6 \% \mathrm{H}_{2}$ gas flowing at $40 \mathrm{ml} / \mathrm{min}$ and followed the same temperature profile: heat to $1500{ }^{\circ} \mathrm{C}$ at $5{ }^{\circ} \mathrm{C} / \mathrm{min} ; 5 \mathrm{~min}$ isothermal; cool to room temperature at $2{ }^{\circ} \mathrm{C} / \mathrm{min}$. Representative dilatometry curves are shown in Figure 75. 
These data sets support MOX and MA-MOX fabrication process development and optimization with regard to sintering rate, pellet density and oxygen stoichiometry.

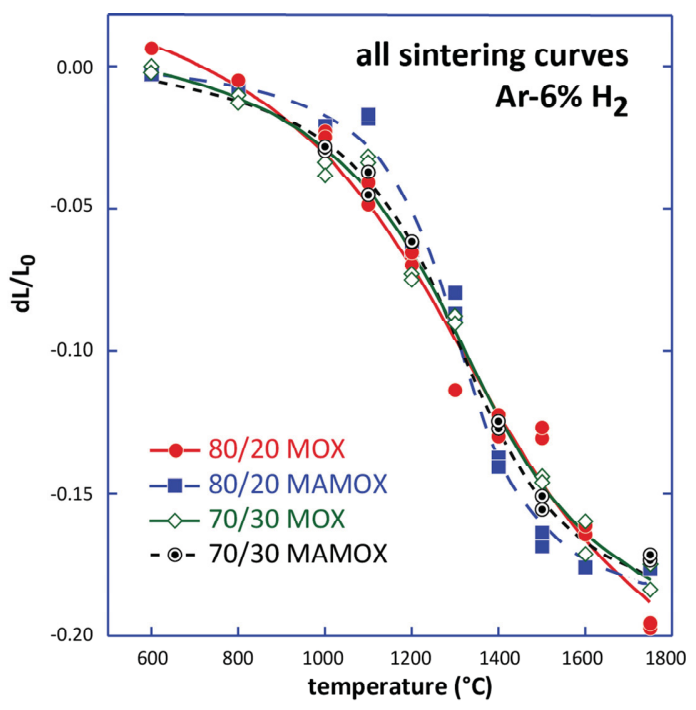

Figure 74. Interrupted sintering curves for pellets heated in $\mathrm{Ar}-6 \% \mathrm{H} 2$.

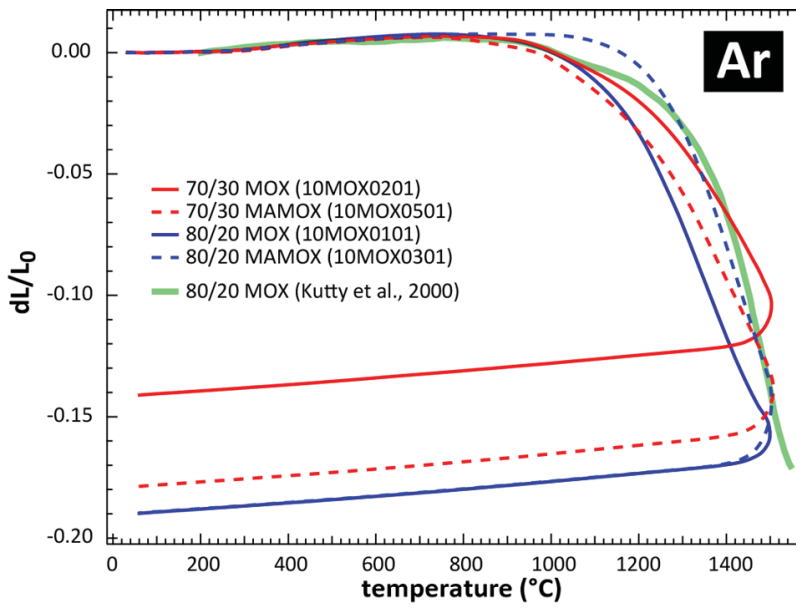

Figure 75. Dilatometry curves of pellets sintering in an Ar atmosphere.

\subsection{In Situ Laser Flash Analysis of Ceramic Fuel Compacts During Sintering}

A. Nelson, atnelson@lanl.gov, LANL

Improving our understanding of the theory underlying sintering and densification of ceramic nuclear fuels is an important component driving next generation fuel fabrication technologies and controls. An ability to better predict the microstructure and final geometry of a fuel pellet as a function of sintering atmospheres, times, and temperatures is necessary for input to fuel performance codes in order to accurately predict the temperature distribution throughout a fuel rod. In parallel to improved modeling capabilities, new experimental techniques must be developed to understand the sintering process at all phases.

Laser flash analysis (LFA) is a standard technique used to measure the thermal conductivity of dense materials. While samples are conventionally of uniform and constant geometry, modifications to fixturing and furnace controls facilitated LFA measurement of the thermal diffusivity of a sample during densification. Data can be taken continuously for a ceramic body as it sinters beginning at room temperature through final densification above $1600^{\circ} \mathrm{C}$. The resulting data is an important companion to conventional dilatometry. Typical results are plotted in Figure 76. 


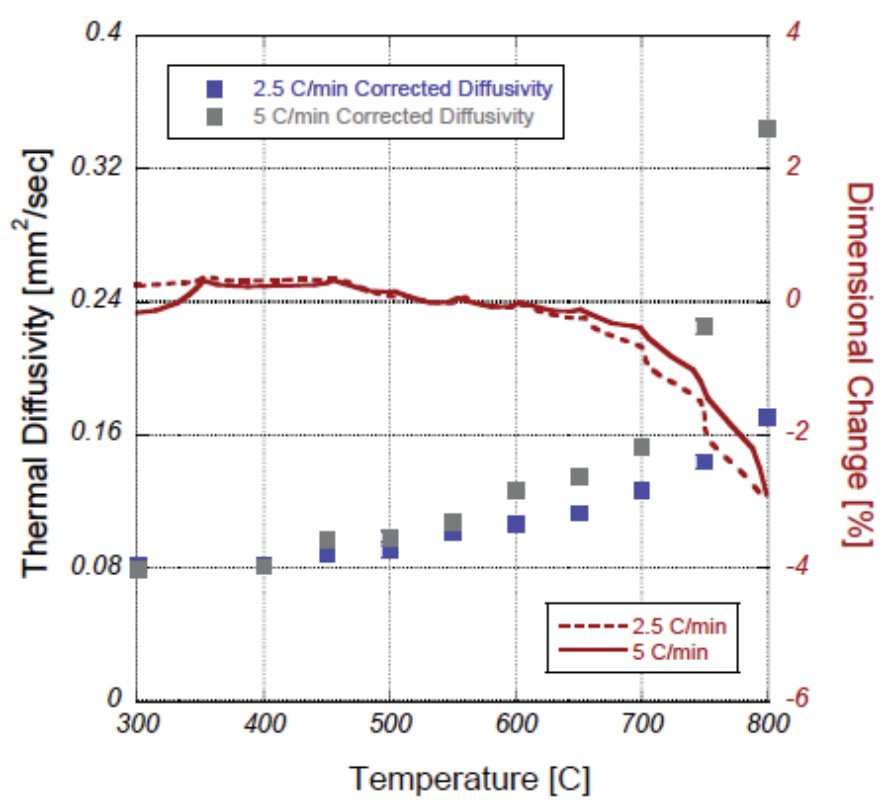

Figure 76. Results from in situ LFA sintering studies performed for UO2 heated at two different rates. The temperature scale is restricted to show only the early stages of sintering to highlight the role of in situ LFA studies compared to data generated using conventional dilatometry.

Figure 76 shows the results of $\mathrm{UO}_{2}$ sintered at two heating rates. The plot shows an increase in the measured thermal diffusivity at temperatures well below the point where the first reduction in length is observed in the dilatometry data. This increase in the thermal diffusivity is caused by the initial necking of powder particles brought about by species transport. Understanding the role of heating rate, atmosphere, and powder morphology on the early stages of sintering and compact densification is an important component that must be captured in models currently under development. Expansion of this technique to include other cations in solution as well as nontraditional powder synthesis routes will greatly aid understanding of their effects on fuel pellet densification.

\subsection{Thermal Transport in Ceramic Fuels}

\section{A. Nelson, atnelson@lanl.gov, LANL}

Thermal transport in ceramic nuclear fuels remains only marginally understood on the level necessary to allow for integration of mechanistic, first principles models into fuel performance codes. The importance of thermal conductivity to reactor operation and safety scenario analysis has historically motivated exploration and development of numerical correlations that seek to link evolution of thermal conductivity to fuel burnup. Though not without utility, these curve fits are limited to very specific application; extrapolation to different reactor conditions or even slightly different fuel chemistry is not possible. Development of new fuel performance codes requires improved understanding of the many factors impacting thermal conductivity of ceramic fuels.

One of the leading causes of degradation in the thermal conductivity of oxide fuels is the addition of cation species beyond uranium alone. The best known example is plutonium as used in MOX, but other solid species can be introduced either intentionally as reactor controls (e.g., gadolinium poison), through carryover during reprocessing, or as fission products during operation. Understanding the role of species' charge and atomic radius on the thermal conductivity of uranium dioxide solid solutions is of immense interest to ongoing first principles modelers seeking to understand these effects. 
Samples can be produced using either conventional mechanical mixing or through advanced techniques. Measurement of the thermal conductivity is executed using laser flash analysis to high temperatures. Figure 77 shows the results of urania-ceria solid solutions with varying ceria content. Ceria is traditionally used as a surrogate for plutonium, but is also an important fission product in its own right. As predicted by theory, increasing the quantity of cerium atoms enhances phonon-defect scattering ( $A$ pre-exponential) while phonon-phonon scattering ( $B$; the slope of the inverse diffusivity) is unchanged.
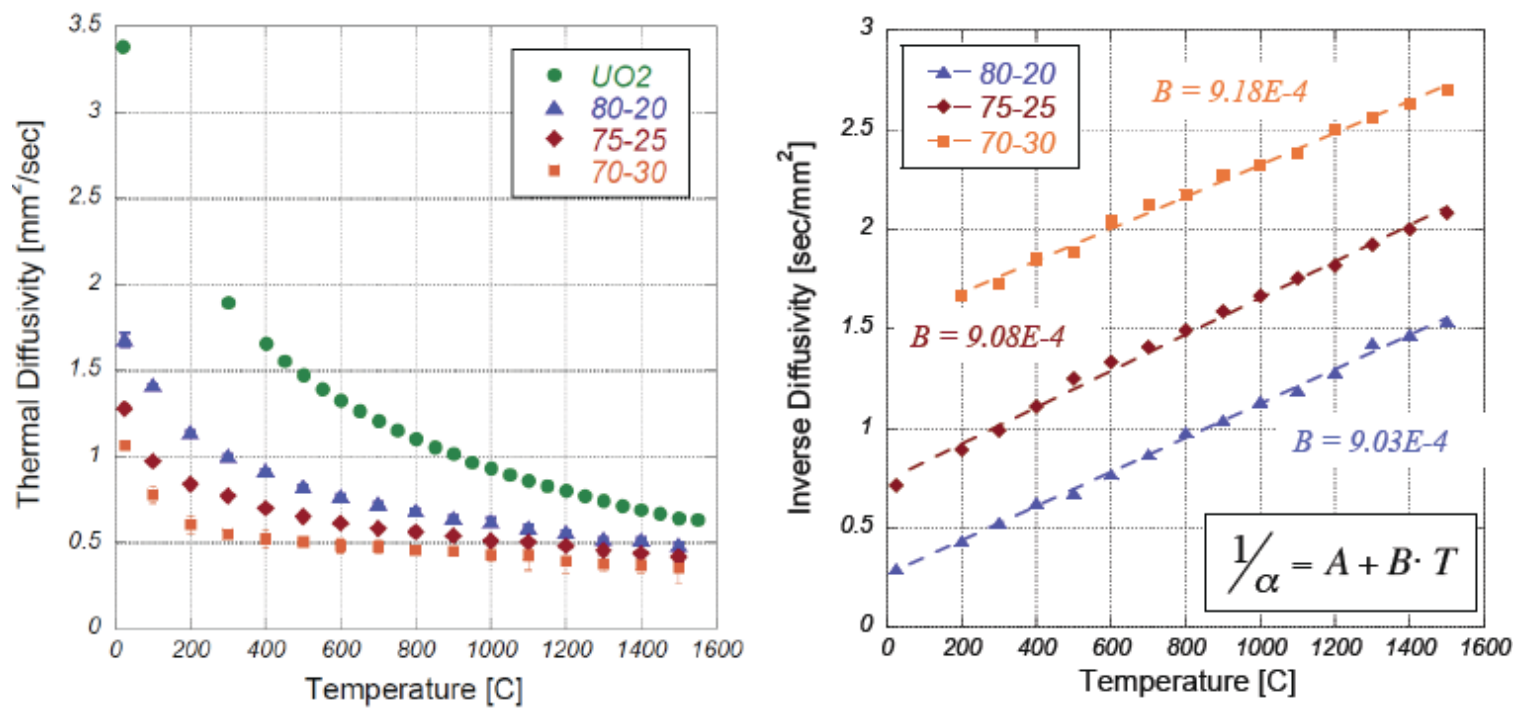

Figure 77. The thermal diffusivity of three urania-ceria solid solutions plotted against temperature. The original data is plotted on the left, while the inverse diffusivity is shown on the right. Plotting of the inverse diffusivity allows for graphical extraction of the pre-exponential factors $A$ and $B$.

Work in this area is currently progressing to study the role of other cations to capture the effect of different charge states as well as the role of oxygen defect structures in restricting thermal conductivity of these systems. With more complete data sets provided in this manner, modelers can continue to develop more accurate representations of phonon scattering in oxide ceramic fuel systems. These results will then directly feed upward to drive development of new and more accurate fuel performance codes.

\section{$5.10 \mathrm{UO}_{2}$ Crystal Growth}

Darrin Byler, dbyler@lanl.gov, James Valdez, Ken McClellan, LANL

Ceramic nuclear fuels are very complicated material systems with impurities (or additions to tailor properties), varying oxygen stoichiometries, not to mention the chemical and microstructural modifications that occur in-pile with the production of fission products. In the interest of distinguishing the effects of individual variable, separate effects testing has been started to evaluate the variations in material properties and determine the effects of fission products and their mobility as a function of variables such as temperature, composition and atmosphere. To improve the ability to characterize a particular contribution, it is important to reduce the number of variables in a system and then systematically evaluate variable changes. To study effects such as fission product mobility at grain boundaries, it is essential to engineer grain boundaries by effectively coupling single grains (crystals) together to form the desired GB or by growing very large grained materials and selectively harvesting grains of interest.

In FY 2011, a series of methods to grow high melt point oxide crystals were evaluated and the "skull" melting method depicted in figure 1a was chosen to grow large $\mathrm{UO}_{2}$ crystals. A high power RF induction unit was sourced; a cold crucible was designed, fabricated and tested using zirconia powder. Tests were 
successful in melting the zirconia at full power and forming large crystals as shown in figure $1 \mathrm{~b}$ below. To improve heating efficiency, the cold crucible was redesigned and tested with zirconia followed by urania. The preliminary growths used a tungsten susceptor to heat and melt a small quantity of $\mathrm{UO}_{2}$ resulting in crystals with a range of sizes between $20 \mu \mathrm{m}$ and $150 \mu \mathrm{m}$ with small voids and imperfections present due to the partial melt. Based on these successes, a transition to larger $\mathrm{UO}_{2}$ crystals can be performed to produce reference materials to feed thermochemical and thermophysical studies in addition to providing materials for separate effects testing.

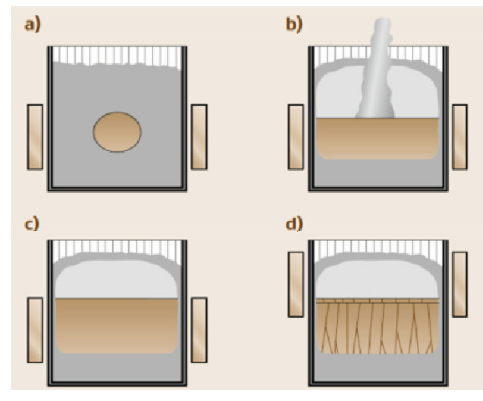

(a)

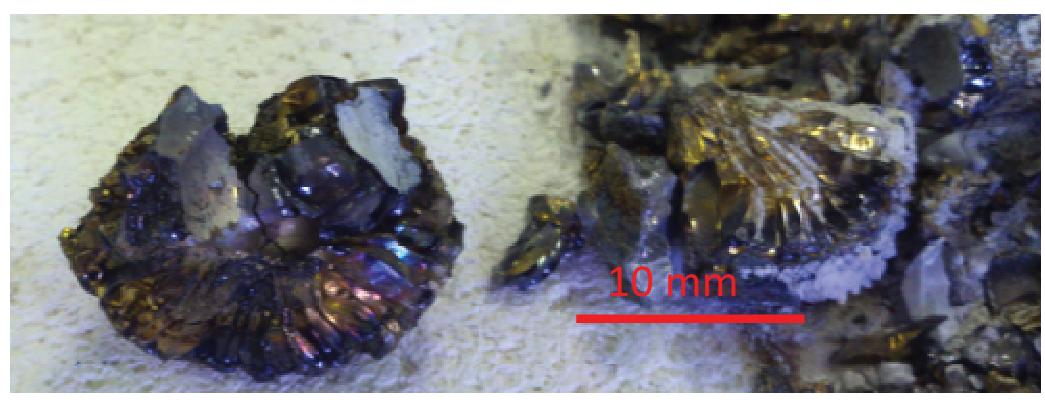

(b)

Figure 78. (a) Illustration of skull melting process illustrating melting in the center of the powder, powder filling into melt, full melt and nucleation and growth of grains; (b) zirconia crystals formed from skull melting test.

An alternate method of crystal growth was conducted in parallel to ensure success of the program. The method uses an exaggerated grain growth technique at $1550^{\circ} \mathrm{C}$ under a controlled atmosphere to cause rapid grain growth while preventing phase changes that could damage the integrity of the pellet. Crystals with grain sizes of approximately $1.5 \mathrm{~mm}$ have been grown using this method indicating the potential to generate large single and multi-crystals via this solid-state method at lower temperatures. By leveraging these methods, materials can be supplied for fundamental property measurements as well as separate effects testing.

\title{
5.11 Uranium Dioxide Films with Embedded Kenon
}

\author{
I.O.Usov, iusov@lanl.gov, LANL
}

Xenon $(\mathrm{Xe})$ is the major fission gas element produced during nuclear fuel burning. High concentrations of insoluble Xe leads to fuel matrix swelling as well as degradation of mechanical properties and thermal conductivity. Release of Xe gas also reduces thermal conductivity in the fuel clad gap and increases pressure in the fuel pin. Thus, both Xe retained in the fuel pellet and Xe released to the plenum are detrimental to the fuel performance and safety and therefore must be well understood and controlled.

Experimental data concerning description of Xe diffusion in $\mathrm{UO}_{2}$ have a wide range of disparity such that it is difficult to verify modeling results for development of predictive nuclear fuel performance codes. A key problem, which relates to obtaining quantitative parameters governing Xe migration, is fabrication of reference $\mathrm{UO}_{2}$ samples, with an uniform and controllable Xe concentration, separated from the various effects attendant to the fission process.

The goal of this work is to fabricate and charcaterize reference depelted $\mathrm{UO}_{2}\left(\mathrm{DUO}_{2}\right)$ samples with controlled microstructure and fission gas distribution. Such reference samples are indispensable for systematic experimental studies elucidating the underlying physics of fission gas behavior. This data will provide an input for modeling of fundamental atomic-scale processes that are active during nuclear fuel burning and in particular support the separate effects testing efforts. Acquisition of such fundamental 
knowledge will lead to a strong foundation for the development of nuclear fuel perfomance theoretical modeling methods and simulation codes.

In fiscal year 2011, the focus was on the fabrciation of $\mathrm{DUO}_{2}$ samples with emebedded Xe in a thin/thick film form by the ion beam assisted deposition (IBAD) technique [1]. The IBAD process consists of material deposition and simultaneous bombardment of a growing film with low energy Xe ions (see Figure 79). Figure 80 shows a photo image of IBAD $\mathrm{DUO}_{2}<\mathrm{Xe}>$ film deposited on $\mathrm{DUO}_{2}$ substrate. To perform a comparative analysis between the film and the substrate microstructure, half of the substrate was shielded during the deposition.

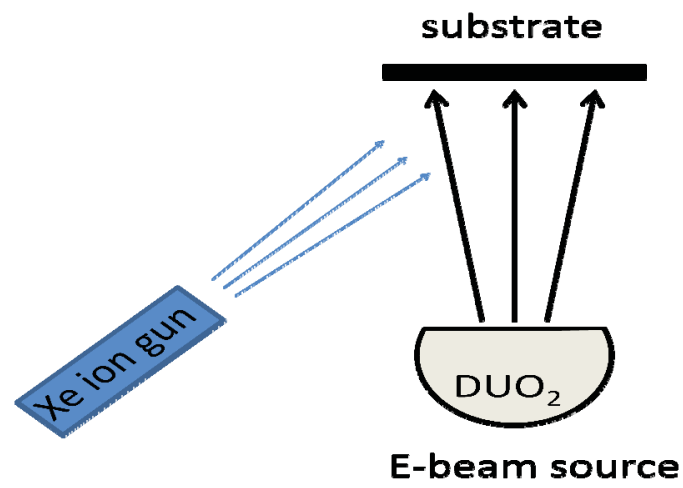

Figure 79. Schematic of IBAD process employed for fabrication of $\mathrm{DUO}_{2}$ film doped with $\mathrm{Xe}$ atoms. Deposition of the $\mathrm{DUO}_{2}$ film is carried out by electron beam (e-beam) evaporation and Xe atoms are incorporated into the growing film by simultaneous bombardment with low energy Xe ion beam.

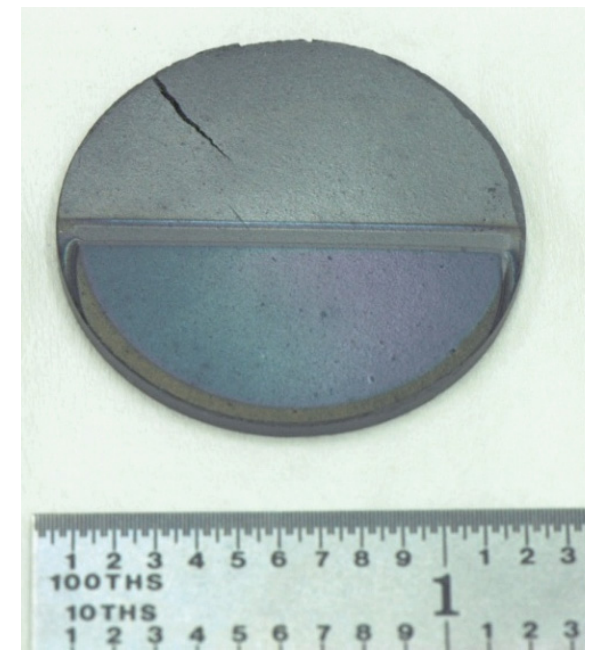

Figure 80. Photo image of the $\mathrm{DUO}_{2}$ substrate coated with a $5.1 \mu \mathrm{m}$ depleted urania film with embedded $X e$ (lower half of sample). The upper half of the sample, containing the crack, is the substrate without the film, which was shielded during the deposition.

We have investigated the effect of deposition conditions, substrate material and post deposition annealing on $\mathrm{DUO}_{2}$ stoichiometry, microstructure and grain size, and also Xe incorporation, redistribution and release from $\mathrm{DUO}_{2}$. We established that as grown films were stoichiometric $\mathrm{DUO}_{2}$ and crystalline.

We discovered that, on the initial stage, $\mathrm{DUO}_{2}$ film is composed of small grains (grain size $\sim 20 \mathrm{~nm}$ ). As the film thickness increases grains grow as well and columnar type microstructure evolves. At the film thickness of $\sim 5 \mu \mathrm{m}$, grain sizes up to $135 \mathrm{~nm}$ were observed. We evaluated a range of analytical techniques for quantitative $\mathrm{Xe}$ analysis. For thick $\mathrm{DUO}_{2}$ films our preference was given to X-ray 
fluorescence and for thin films to Rutherford Backscattering Spectroscopy (RBS). Figure 81 shows an example of RBS analysis applied to a study of Xe behavior in thin $\mathrm{DUO}_{2}$ film. The $\mathrm{DUO}_{2}<\mathrm{Xe}>$ film was deposited on silicon carbide substrate and coated with alumina layer to preserve $\mathrm{O} / \mathrm{U}$ ratio. The amount of $\mathrm{Xe}$ in the as-grown sample was estimated to be $\sim 0.6$ at $\%$. No Xe redistribution or release was observed after annealing in ultra high purity argon at temperatures up to $1000{ }^{\circ} \mathrm{C}$.

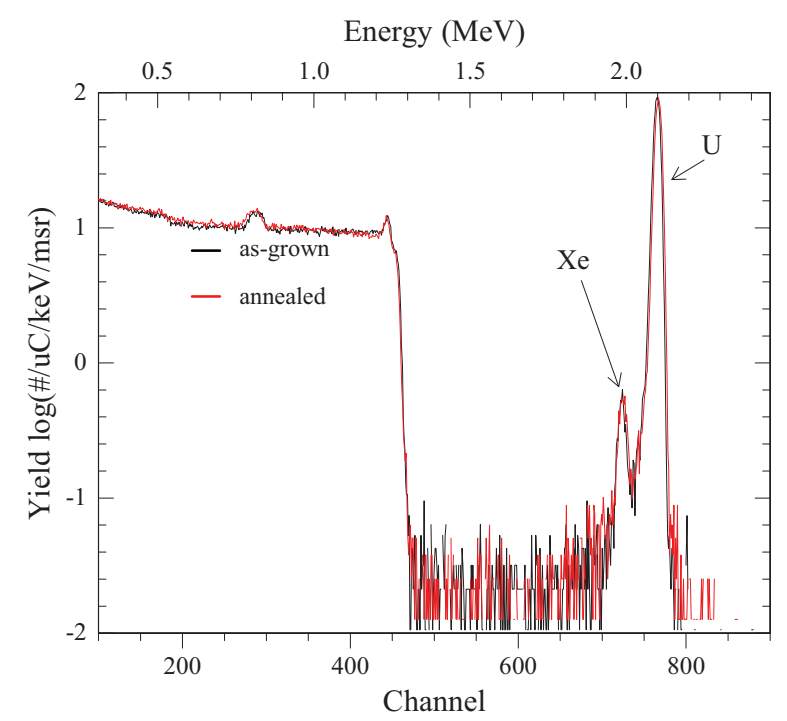

Figure 81. RBS spectra obtained from the UO2<Xe $>$ film deposited on silicon carbide substrate and coated with protective alumina layer. Peaks corresponding to backscattering from $U$ and $X e$ atoms are labeled on the graph. Features at channel numbers below 500 correspond to backscattering from Si, Al, $O$ and $C$ atoms. We did not observe any changes in RBS spectra before and after annealing.

\section{Reference}

1. I.O. Usov, J.Won, D.J. Devlin, Y.-B. Jiang, J.A. Valdez, K.E. Sickafus “A novel method for incorporating fission gas elements into solids”, J. Nucl . Mater. 408 (2011) 205.

\subsection{Thoria Feedstock Procurement and Authorization of Experimental Operations}

A. Nelson, atnelson@lanl.gov, LANL

Thorium nuclear fuel forms have long been of interest given the lack of TRU waste produced by a thorium cycle. Although this is an important consideration, researchers in recent years have began to emphasize the wide array of other beneficial material properties possessed by thorium dioxide (thoria) in comparison with the more familiar uranium dioxide fuels as utilized in the existing reactor fleet. Foremost of these benefits are improved thermal conductivity, mechanical property behavior, and fission gas retention. Additionally, thoria is a more stable chemical form than urania. Thoria cannot oxidize further even when provided with an excess of oxygen at high temperatures, whereas this is a significant concern for urania. Thorium dioxide performance in accident scenarios may therefore be appreciably enhanced and could allow for a more accident resilient 'drop in' fuel form for use in current reactors.

Work within the FCRD program in FY 2011 has focused on 1) procurement of a $50 \mathrm{~kg}$ batch of thoria powder as a reference feedstock, and 2) providing a programmatic capability to fabricate and characterize thorium containing ceramic materials. Authorization was established at LANL to process thorium using the same capabilities available for uranium. Thorium dioxide powders can be characterized, pressed into compacts, sintered to high density, characterized for chemistry and microstructure, and undergo 
thermophysical property characterization to over $2000^{\circ} \mathrm{C}$. The chemistry-structure-property relations constructed using these capabilities will facilitate improved accuracy in reactor loading and burnup calculations necessary to evaluate candidate fuel form designs and fuel cycle options.

\subsection{Vented Fuel/Getter Concept}

J. Valente, valente@bnl.gov, G. Greene, M. Todosow, J. Wishart, J. Hatcher, BNL; C. Scherer, LANL

To extend the burnup capability of nuclear fuel, candidate getter materials, which have the capacity for excess adsorption, will be examined. This means that the volume occupied by the getter results in more retained adsorbate in that occupied volume than adsorbate would occupy in the same volume without the presence of the getter material (assuming the same adsorbate temperature and pressure conditions). The goal is to produce a getter material that preferentially captures and retains fission gases under operating, transient and accident conditions (see Figure 82).

Preliminary results indicate that $\mathrm{Pd}$ powder particles hold strong promise, as they exhibit chemisorption for Xe. Other carbon based getter materials do show physisorption properties for Xe, but these characteristics would be less rewarding than those having chemisorption.

If the results for Pd hold true, then a reduction in the fuel pin's internal gas pressure caused by fission product gases such as Xe, would be seen. Experimental results and calculations show that Pd would be able to adsorb on the order of 100 times the moles of Xe in the same space as the free plenum volume at the same temperature and pressure conditions (400C and one atmosphere).

Future efforts include investigating the validity of the Pd results, and that of other candidate materials. These materials include $\mathrm{Ni}$ and $\mathrm{W}$ as they have properties that are also expected to result in chemisorption of $\mathrm{Xe}$.

In addition, the chemisorption process would strongly assist in the desire to enhance the mitigation of internal pressure fuel pin failures that might occur during accident conditions, as well as extending fuel pin burnup.

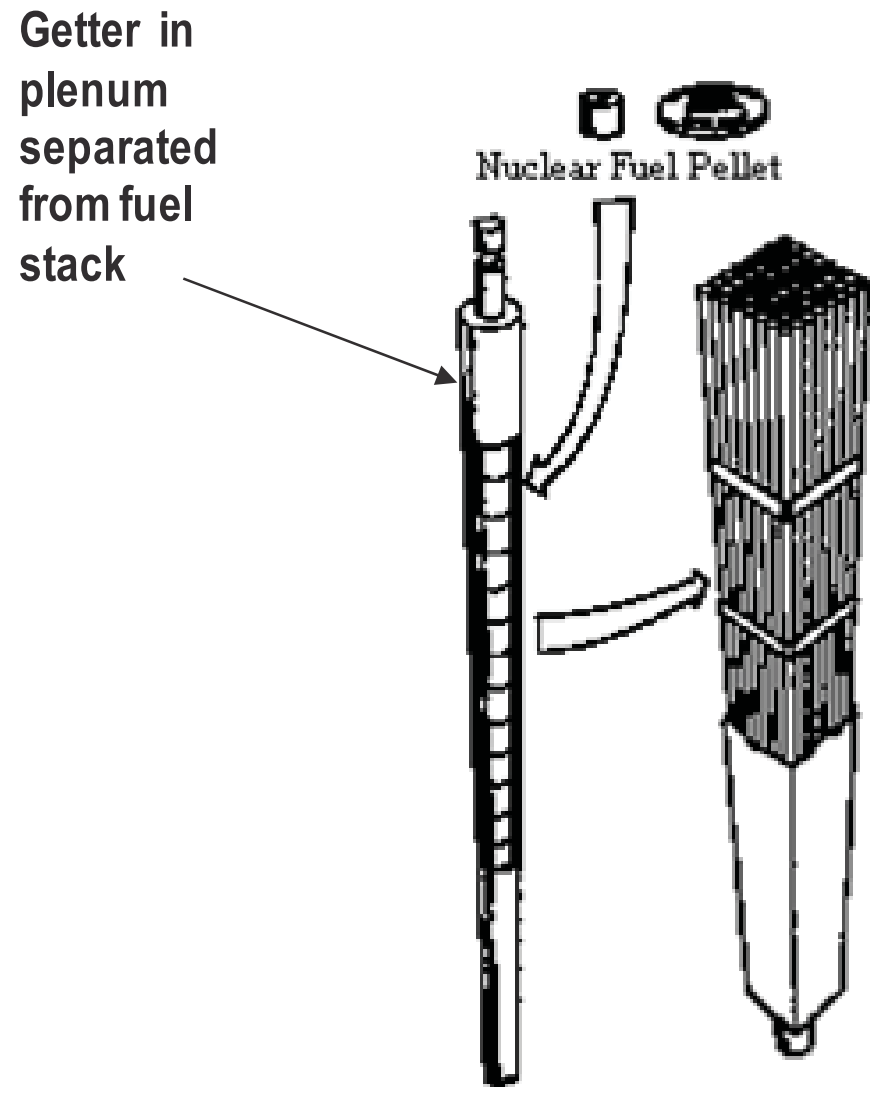

Figure 82. Getter in plenum separated from fuel stack. 


\section{GOATED PARTIGLE FUELS TECHNOLOGIES}

Technical Lead: L. Snead, snead1l@ornl.gov, ORNL

Coated Particle Fuels Technologies focused on the application of coated particle fuel (CPF) technology for advanced reactor platforms including LWR applications. It is highly integrated with direct modeling, fuel fabrication, irradiation testing, and post irradiation performance evaluation. FY 2011 focus areas included modeling, TRU TRISO Development, LWR Microencapsulated fuel development, and the testing of advanced clad and fuel materials being developed for enhanced safety under the FCRD and other programs.

Some of the specific accomplishments include the fabrication and assembly of a new coating furnace for use in fabricating microencapsulated fuel particles and the fabrication of the first uranium bearing FCM fuel. This includes an intensive program for optimizing the matrix for this fuel system. FCM originally designed for uranium-burn or TRU deep-burn in LWRs, was analyzed for use in fully loaded LWR Cores.

An irradiation vehicle was designed for HFIR irradiation of fueled and surrogate FCM fuel in contact with zircaloy cladding to simulate LWR application. Metal Matrix Microencapsulated (M3) fuel was designed for increasing uranium utilization in LWRs. Fuel rods were fabricated with surrogate TRISO particles with various most outer layer coatings of pyrolytic carbon (PyC), SiC, and zirconium carbide $(\mathrm{ZrC})$.

Irradiation of a series of FCM fuel pellets clad in Zircaloy-4 and M3 fuel rods (containing TRISO particles with surrogate kernels) was started in HFIR. All the fuel specimens conformed to the exact geometry of the Westinghouse $17 \times 17$ PWR bundles. First round of PIE has begun with encouraging results as to the integrity of the fuel matrix under neutron irradiation.

The assembly and demonstration was completed of a Focused Ion Beam and Simultaneous Thermal Analyzer for use on fuels for the wider FCRD program.

A process was developed and demonstrated to add $\mathrm{SiC}$ to the $\mathrm{UO}_{2}$ kernel. This milestone is on a path to verify a FY 2010 modeling milestone, which proposed this route to mitigating high burnup kernel migration, a long-standing issue for microencapsulated fuels. Incorporated $\mathrm{SiC}$ and graphite matrix physical properties into FRAPCON and performed a preliminary analysis to assess fuel temperatures under LWR operating conditions.

Accident Testing was conducted on FCM Fuel and Advanced Fuel and Cladding Materials. Two sets of tests were performed to provide insight into the performance of fuel/cladding materials under severe accident conditions (high temperature steam attack). A side-by-side comparison of different classes of materials is being developed through examination of a wide matrix of materials including zirconium alloys, $\mathrm{SiC}$ components, advanced fuel forms, as well as conventional and advanced ferritic and austenitic steels.

\subsection{Fuel Performance Modeling}

M. Pope, mike.pope@inl.gov, A. Ougouag, INL

A mesoscale kinetic Monte Carlo (KMC) model developed to investigate the diffusion of silver through the pyrolytic carbon and silicon carbide containment layers of a TRISO fuel particle has been developed. The release of radioactive silver from TRISO particles has been studied for nearly three decades, yet the mechanisms governing silver transport are not fully understood. This model atomically resolves Ag, but provides a mesoscale medium of carbon and silicon carbide, which can include a variety of defects including grain boundaries, reflective interfaces, cracks, and radiation-induced cavities that can either accelerate silver diffusion or slow diffusion by acting as traps for silver. The key input parameters 
to the model (diffusion coefficients, trap binding energies, interface characteristics) are determined from available experimental data, or parametrically varied, until more precise values become available from lower length scale modeling or experiment. Some predicted results, in terms of the time/temperature dependence of silver release during postirradiation annealing are seen in Figure 83, and the variability of silver release from particle to particle have been compared to available experimental data from the German High-Temperature Reactor (HTR) Fuel Program.
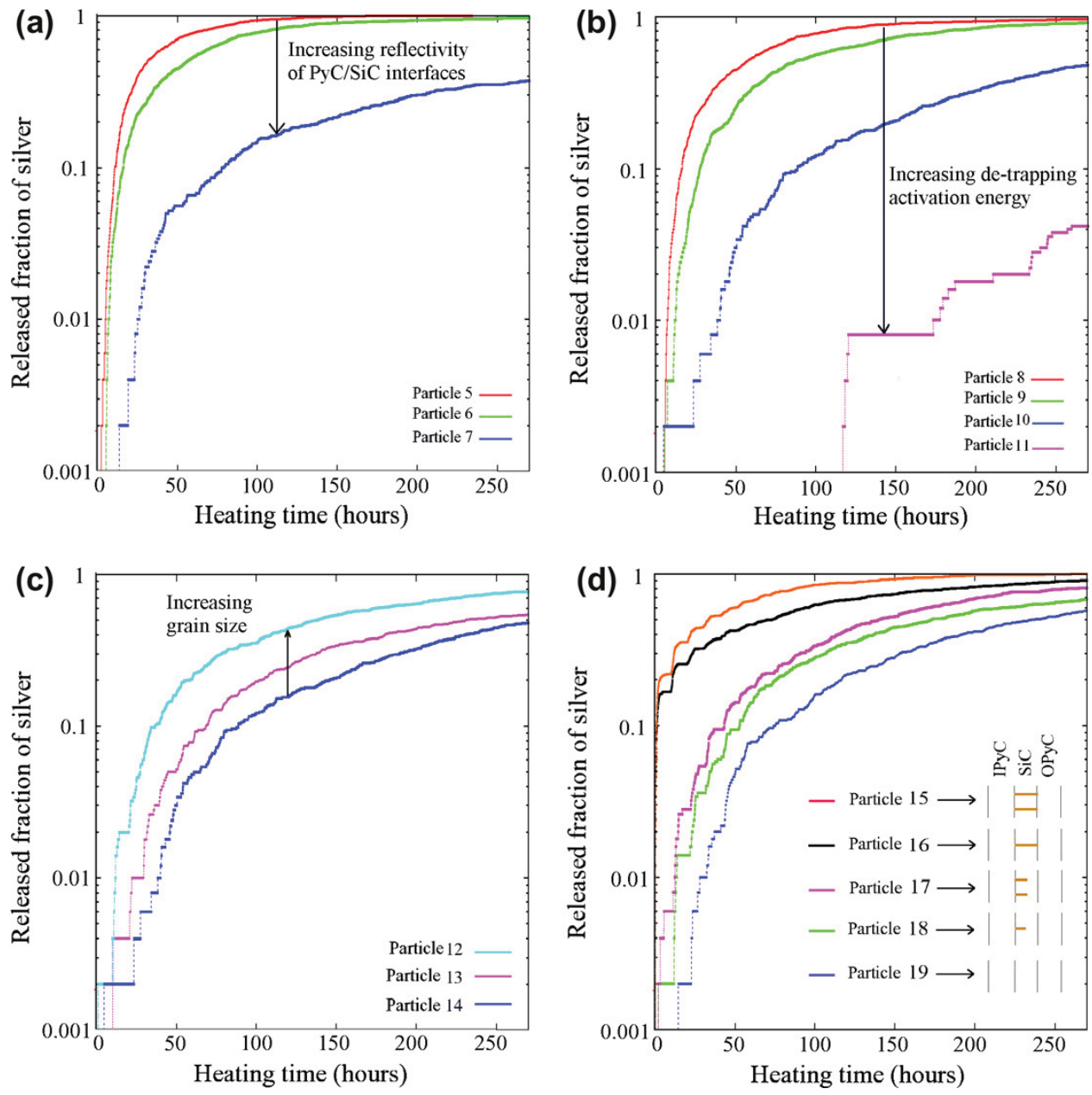

Figure 83. Kinetic Monte Carlo simulations of the fractional release of silver at $1700^{\circ} \mathrm{C}$ : a) influence of the reflective nature of the PyC/SiC; activation energies for particles 5, 6 and 7, respectively $(0 \mathrm{eV}, 0 \mathrm{eV}, 0$ $\mathrm{eV}),(0 \mathrm{eV}, 2 \mathrm{eV}, 1 \mathrm{eV})$ and $(0 \mathrm{eV}, 2 \mathrm{eV}, 2 \mathrm{eV}) ; \boldsymbol{b})$ influence of the cavity de-trapping activation energy with a given cavity number density of $10^{18} \mathrm{~m}^{-3}$ in SiC. Particle 8 has no cavities, whereas 9,10 and 11 have de-trapping activation energies of $2.7 \mathrm{eV}, 3.0 \mathrm{eV}$ and $3.3 \mathrm{eV}$, respectively; c) influence of the grain geometry in SiC; particle 12 has no grains, 13 has square $1 \mu \mathrm{m}$ grains, 14 has rectangular grains $1 \mu \mathrm{m}$ in height and a range of width from $1 \mu \mathrm{m}$ to $40 \mu \mathrm{m}$; d) influence of the cracking within the SiC layer, cracks are $20 \mathrm{~nm}$ wide and provide instantaneous transport path for incident Ag atoms.

The effect of defects on the thermal conductivity of silicon carbide was studied at $800 \mathrm{~K}$ with the Green-Kubo method. Six defects were studied, carbon vacancy (V_C), silicon vacancy (Si_C), carbon interstitial (I_C), silicon interstitial (I_Si), carbon anti-site (C_Si), and silicon anti-site (Si_C). The thermal conductivity was studied over a wide defect concentration range from $1 \mathrm{defect} / 51 \overline{2}$ atoms to 1 defect/4096 atoms. Figure 84 plots the relative increase in thermal resistivity $((\rho-\rho) / \rho o)$ of the 6 defects 
as a function of concentration, where $\rho$ is the resistivity of the defects and $\rho$ o is the resistivity of the perfect system. The resistivity is the inverse of the thermal conductivity.

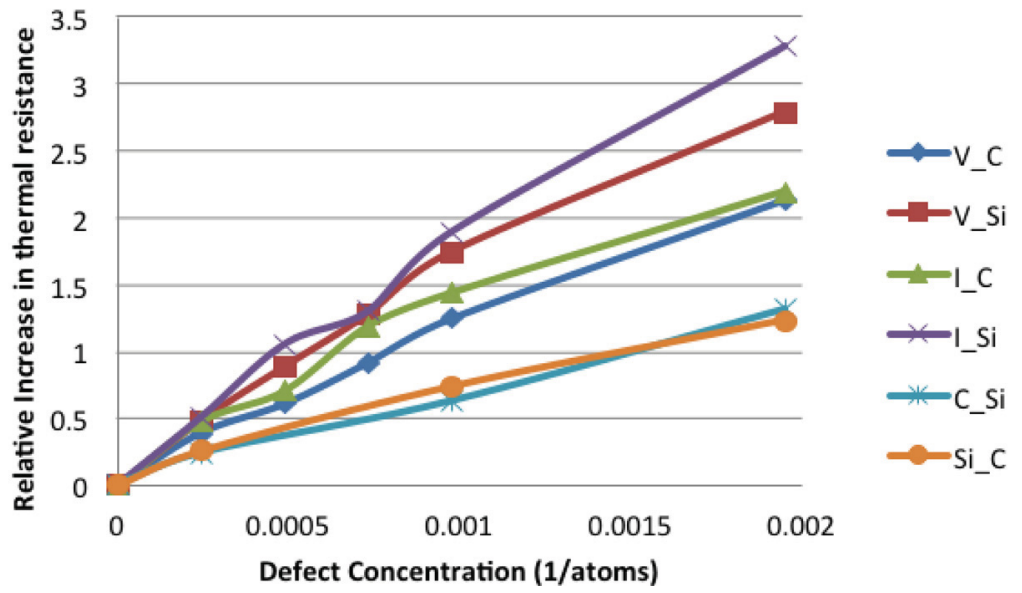

Figure 84. Increase in thermal resistance as a function for defect concentration for six different types of defects.

As noted in Figure 84, as the defect concentration increases, the thermal resistivity increases. Si interstitials have the largest effect on thermal conductivity followed by $\mathrm{Si}$ vacancies. The next most influential defects are the carbon interstitial and vacancy. This is followed by both anti-sites which have virtually the same effect on the thermal conductivity. In addition, the plots are extended to a perfect crystal and a linear regime is found between the results and the perfect crystal. This method has been compared to experimental work and found reasonable agreement. With these results and knowledge of defect concentration in irradiated $\mathrm{SiC}$, initial estimates can be made to the reduction of the thermal conductivity. These results are being prepared for publication.

The development of high burn-up fuels relies on the incorporation of TRISO particles into a silicon carbide matrix (FCM) that exhibits high thermal conductivity. Due to differences in the properties of the various constituents making up the TRISO particles, as well as those of the $\mathrm{SiC}$ matrix, modeling and experimental studies are being conducted to assess the factors that influence the generation of stresses and the fuel performance. COMSOL Multiphysics software was used to predict the residual stresses developed within a TRISO particle embedded in a SiC matrix that result from mismatch in the thermal and mechanical properties of the constituents. This model showed that debonding of the zirconia particle during cooling could occur as a result of the tensile stresses generated at the interface. The debonding event was also modeled by including a thin layer between zirconia and the $\mathrm{C}$ buffer layer, which initially had elastic and thermal properties identical to zirconia. When the tensile stresses exceeded a critical value, the elastic modulus was taken reduced by several orders of magnitude, which has the effect of relaxing the stresses on both the zirconia particle and the $\mathrm{C}$ buffer layer. There was some concern regarding the potential for damage due to the stresses imposed during hot pressing of TRISO particles$\mathrm{SiC}$ matrix powder mixture; however, analytical results show that the tensile stress levels acting on the TRISO particles are negligible.

The effect of particle-particle interactions on the matrix stresses was also examined by first using the aforementioned model to estimate the effective elastic and thermal properties of the composite particle system. A single particle having these effective properties was then used to examine the effect of particleparticle spacing upon the residual stresses developed within the matrix. As shown in Figure 85, the tensile stresses in the matrix increased from $3 \mathrm{MPa}$ to $47 \mathrm{MPa}$ as the particle spacing decreased from 4 to 1 . This model is currently being used in conjunction with typical fracture properties for $\mathrm{SiC}$ matrix materials to 
estimate the probability of matrix cracking. The models described above were based on the assumption of uniform particle sizes and simple geometric arrangement of particles within the matrix.

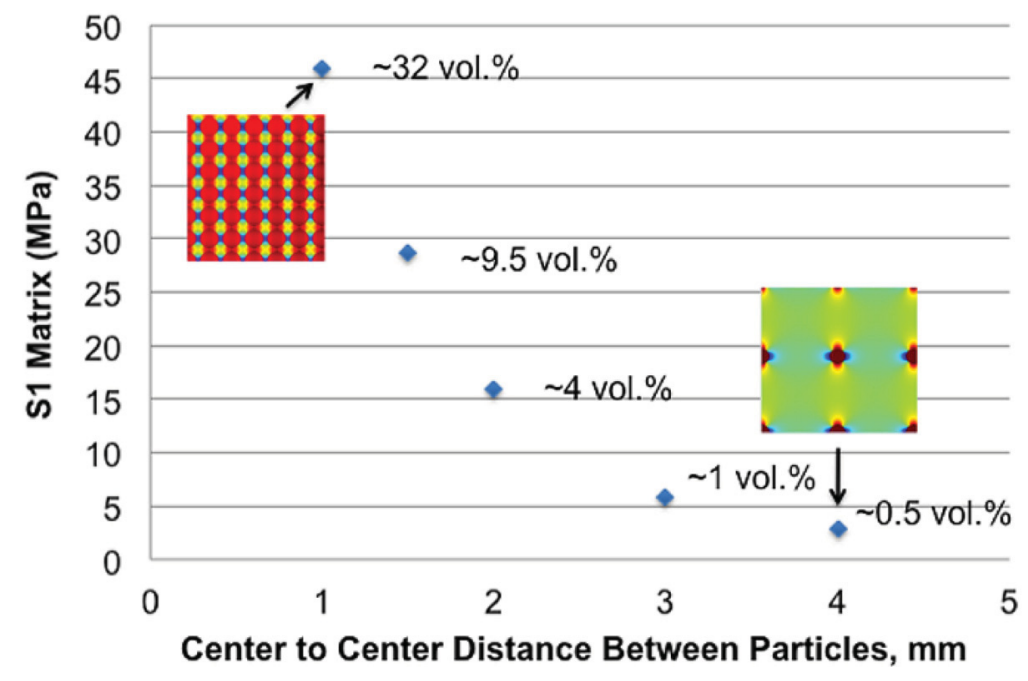

Figure 85. The tensile stress generated within the SiC matrix as a result of interactions between the stress fields associated with each TRISO particle arranged in a simple cubic array increases rapidly as the inter-particle spacing decreases (i.e., particle loading increases).

\subsection{Thermochemical Data and Model Development}

\section{Snead, sneadll@ornl.gov, ORNL}

Significant progress has been made in developing thermochemical models for oxide fuel kernels for CPF. A compound energy formalism model for the fluorite structure fuel phase has been created and subsystems for uranium and plutonium, and the REs Ce and La, have been completed and included in the model. Adequate models already exists for the white noble metal alloy phase and are included in the database. Secondary oxides phases have been included in the database, but the simple models for the complex phases will require refinement. The addition of $\mathrm{SiC}$ or $\mathrm{ZrC}$ as getters was considered, and results confirm their ability to limit oxygen potential and mitigate kernel migration. Future computational and experimental efforts for TRU fuel will need to focus on further improving the oxide fuel data and models, and address issues such as controlling palladium attack on the $\mathrm{SiC}$ layer.

Work has been initiated in considering complex models for the nitride phases in support of the new effort in CPF related to nitride kernel fuels. Similar to that for the oxides, complex nitride fuel phase models will need to be generated to understand evolution of nitrogen pressure during burnup. A model for the $\mathrm{U}(\mathrm{C}, \mathrm{N})$ phase has been developed allowing calculation of nitrogen pressure above the phase as a function of temperature and composition, and an example of the results can be seen in Figure 86. In addition, understanding the thermochemistry will also impact controlling palladium attack of the SiC coating layer, which will be an issue for nitride fuels as it is for oxide fuels. A targeted computational and experimental effort was outlined to develop data and models that will allow predictive determination of thermochemical behavior to help design high burnup fuels and provide input to fuel performance codes. 


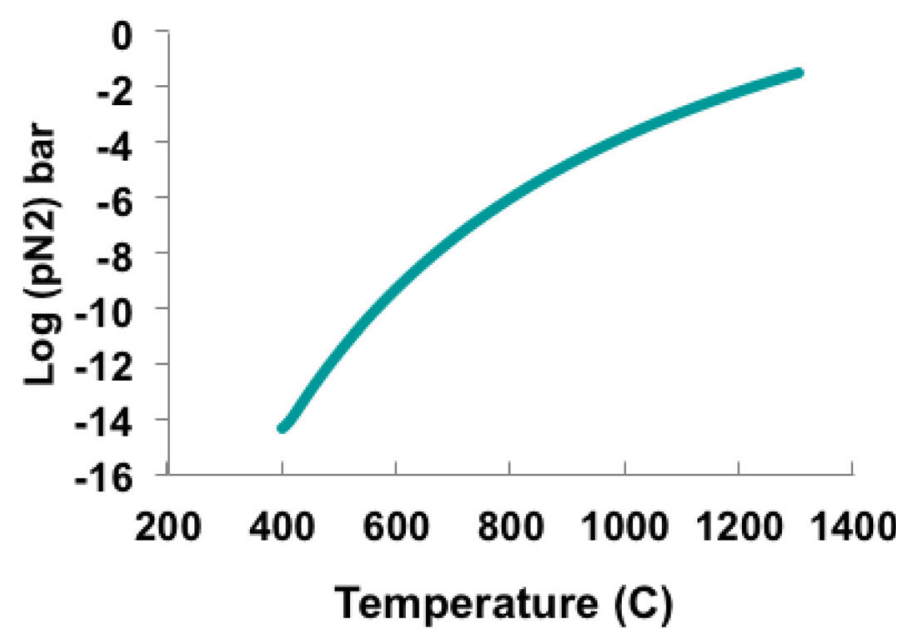

Figure 86. Computed nitrogen pressure over $U C_{0.5} N_{0.5}$ as a function of temperature.

\subsection{TRU TRISO Development}

L.Snead, sneadll@ornl.gov, ORNL

The ultimate objective of this work is the development of CPF for TRU waste destruction in support of the MOC approach to managing the nuclear fuel cycle. Owing to the high burnup capability of the ceramic TRISO fuel form, a plutonium bearing fuel kernel has the potential for use in the management utilization - destruction of TRU material recovered from commercial LWR used fuel. Specific objectives for FY 2011 were to:

1. Secure operational approval and then demonstrate fabrication $\mathrm{Pu}-239 / 3.5$ at $\% \mathrm{~Np}-237$ oxide fuel kernels

2. Complete fabrication of a new coating furnace

3. Develop a plan for continuation of work to install coating equipment in a glovebox as required to coat TRU kernels to produce TRU TRISO

4. Begin PIE of fuel compacts from the FTE-13 irradiation experiment focusing on deep burn relevant aspects of the fuel.

An additional objective was to develop and demonstrate a method to add $\mathrm{SiC}$ to a $\mathrm{UO}_{2}$ kernel as method to enable high burnup. This work was part of an effort to verify an FY 2010 modeling milestone that proposed this route to eliminate high burnup kernel migration, a long-standing issue for microencapsulated fuels.

\subsubsection{TRU Kernel Development}

In FY 2010, two glove boxes were installed in Lab 109 of Bldg. 7920, Lab 109 at the Radiochemical Engineering Development Center at ORNL. The equipment needed to fabricate TRU bearing kernels using the internal gelation process was installed and demonstrated using surrogate materials. In FY 2011, a variety of work control documents, hazard analyses, and operating procedures were completed/approved, and authorization to use the sol-gel glove box for actinide operations was secured on June 29, 2011. However, production of Pu-Np oxide kernels was not to be. Owing to evolving changes in AFC priorities the scope and schedule of the Deep Burn project was changed and emphasis was shifted from the development of high burnup TRU fuel (for use in a TRU burning high temperature gas-cooled reactor as a candidate element of a MOC to the R\&D of a fully ceramic matrix microencapsulated fuel form with the potential to support a variety of reactor applications (e.g., molten salt cooled reactors, 
advanced LWRs, etc.). In January 2010, ORNL received notification of a DOE-NE management concern "about contaminating new facilities and boxes as part of the Deep Burn program objectives" and was directed to not start any Pu work in the new kernel boxes without explicit approval from DOE-NE. Therefore, there are no plans to introduce Deep Burn related TRU materials into the sol-gel glove boxes until further direction is received from DOE-NE. The milestone to demonstrate fabrication of $\mathrm{Pu}$ 239/3.5at\%Np-237 oxide fuel kernels was deferred to FY 2012 and we expect the milestone to be canceled in early FY 2012.

In FY 2010, thermochemical modeling indicated the addition of $\mathrm{SiC}$ to a $\mathrm{UO}_{2}$ kernel could be used as an oxygen getter $\left(\mathrm{SiC}+\mathrm{O}_{2}=\mathrm{SiO}_{2}+\mathrm{C}\right.$ ) to control the oxygen potential (oxygen released from fission of $\mathrm{U}$ in $\mathrm{UO}_{2}$ ) at high burnup and thereby eliminate kernel migration, a long-standing issue for microencapsulated fuels subjected to deep burn. In FY 2011, fabrication of uranium fuel kernels with 4 mole \% SiC nanoparticles was successfully demonstrated using the internal gelation process. Thermochemical calculations indicate a minimum of 4 mole $\% \mathrm{SiC}$ is needed to consume the oxygen that is generated during full burnup. However, testing indicated a fraction of the $\mathrm{SiC}$ is lost during the sintering process, so uranium microspheres with 8 mole \% $\mathrm{SiC}$ were subsequently prepared and are currently being characterized. The results to date have been included in a manuscript that will be submitted to the Journal of Nuclear Materials in FY 2012.

\subsubsection{TRU Goating Development}

A new particle coating furnace, identical to the existing surrogate and uranium coaters currently in service at ORNL, was fabricated from the existing design drawings. The new furnace was assembled in the surrogate coating lab and is being stored there awaiting DOE decisions regarding continuation of the TRU particle coating R\&D activities. A photograph of the new furnace is provided in Figure 87. In addition, the design of the custom coating glove box was completed and a procurement package (specifications, drawings, etc.) was prepared, but procurement was not initiated owing to the aforementioned programmatic redirection.

Finally, a letter report was issued on June 30, 2011 (M21AF080203) documenting the work performed to date on a new TRU particle coating facility in Bldg. 3525 (an ORNL non-reactor nuclear facility) and summarizing the plan for completing the work, including cost and schedule (24 months, $\$ 2 \mathrm{M})$.

It should be noted that a significant amount of work on the TRU coating laboratory has been performed and work was curtailed at appropriate hold points so as to avoid re-work and minimize the cost of resuming the effort. Fortunately, funding was identified from a DOE Oak Ridge Operations Office (DOE-ORO) infrastructure improvement project and was used to continue a portion of the facility engineering and infrastructure work. The managers of the facility improvement funding agreed that completing the design and installation of the glove box ventilation system and procuring standard glove boxes that could support multiple programs was within the scope of their effort. As a result, the refurbishment of the laboratory space has progressed and the new glove box laboratory is currently scheduled for completion in mid FY 2012. 


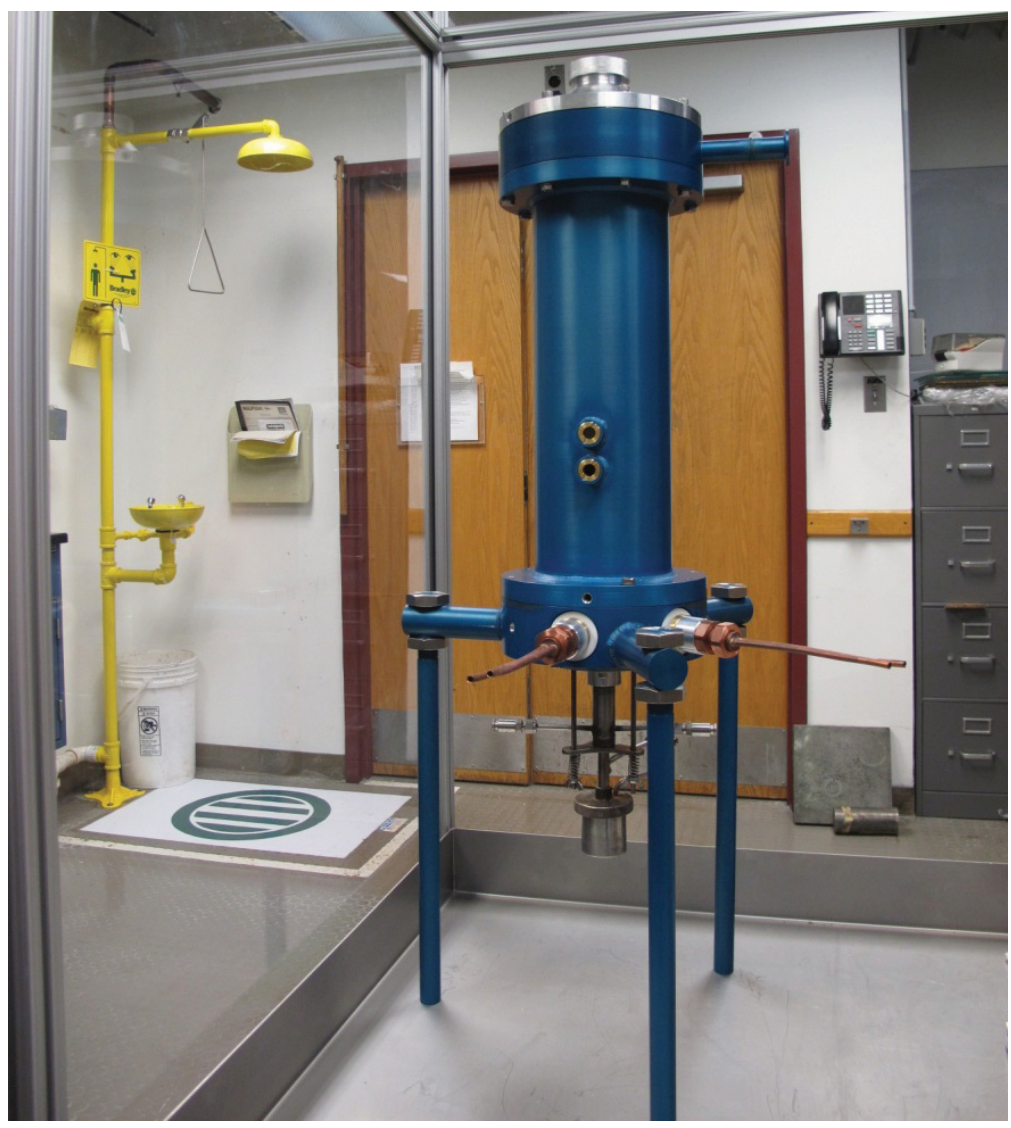

Figure 87. Photograph of the new particle coating furnace.

\subsubsection{FTE-13 Fuel Post-Irradiation Examination}

In the 1970s TRISO-coated $\mathrm{ThO}_{2}$, (Th,Pu) oxide, and $\mathrm{PuO}_{2-\mathrm{x}}$ kernels were irradiated to $\sim 70 \%$ burn-up in the Peach Bottom High Temperature Gas-Cooled Reactor FTE-13 irradiation test, and some of the test articles were archived at ORNL. Samples of this fuel were selected for examination using modern PIE techniques to assess deep burn relevant aspects of their condition, specifically, examination for evidence of kernel migration, general coating behavior (e.g., cracks, shrinkage, debonding, etc.), corrosion of the $\mathrm{SiC}$ layer by noble metals, and irradiated microsphere gamma analysis to detect failed particles. A fulllength compact was selected and deconsolidated, and a 500 micron sieve was used to remove the large diameter thorium particles. Two "grid" type metallographic (MET) mounts of $\mathrm{PuO}_{2-\mathrm{x}}$ TRISO particles were prepared by mounting the selected particles with epoxy in a phenolic base mount. These mounts were then ground flat and polished. One MET mount contains only a few particles in order to reduce the radioactivity for wavelength dispersive spectrometry (WDS) and EDS analysis; the other has tens of particles so that good statistical results can be obtained. The MET mounts were checked by optical microscopy and were found to be suitable for SEM examination. Elemental analyses of the particles using SEM/EDS/WDS is currently scheduled for FY 2012. 


\title{
6.4 Light Water Reactor Fully Ceramic Fuel Development
}

\author{
L. Snead, sneadll@ornl.gov, ORNL
}

\subsubsection{FGM Fuel Fabrication with Surrogate TRISO Particles and Preparation of Surrogate FGM Pellets for HFIR irradiation}

FCM fuels consist of TRISO fuel particles embedded in a silicon carbide ( $\mathrm{SiC}$ ) matrix. Under the FCM fuel concept the graphite matrix of High-Temperature Gas-Cooled Reactor (HTGR) fuel is replaced with a SiC matrix to offer the following advantages for a fuel intended for LWRS: i) improved irradiation stability [1,2], ii) presence of another effective barrier to fission product release, iii) environmental stability under operating (steady state and transient) conditions as well as long term storage, and finally iv) proliferation resistance. In the meantime the high thermal conductivity of the graphite matrix is matched by $\mathrm{SiC}[1,3]$.

Multiple FCM fuel pellets with surrogate TRISO particles have been fabricated to demonstrate the feasibility of production. Some of the fabricated pellets have also been incorporated into irradiation capsules and are currently undergoing irradiation in HFIR cycle 346. The TRISO particle in the FCM fuel consists of a spherical kernel that is coated with multiple layers of carbon and a SiC layer. For the purposes of the first irradiation test the kernel is made from zirconium oxide that yields a surrogate TRISO particle; that is no fissile or fertile material is present in the particle. In the meantime, pellets with 19.7\% low enriched uranium TRISO particles have been fabricated in FY 2011 under this effort.

The powder mixtures utilized during FCM fuel fabrication consisted of SiC nanopowder with addition of yittria, alumina, silica, and occasionally gadolinia nanopowders. The mean diameter of all the powder particles was $30-40 \mathrm{~nm}$. Thorough mixing of the oxide additives with the base $\mathrm{SiC}$ powder is necessary to achieve optimized matrix microstructures. In this study addition of dispersant agents (PEI) along with ultrasonic mixing was used to effectively mix the powders.

Surrogate TRISO particles used during the fabrication of FCM pellets are the $\mathrm{ZrO} 2-500-\mathrm{AK} 2-$ Surrogate TRISO batch that have been previously fabricated and characterized in detail. AK2 particles consist of a $530 \mu \mathrm{m}$ zirconia kernel that is coated to result in a $916 \mu \mathrm{m}$ TRISO particle. AKT-2 Surrogate TRISO particles were embedded in a SiC matrix by hot-pressing a powder-particle mixtures at $1850{ }^{\circ} \mathrm{C}$ and $15 \pm 5 \mathrm{MPa}$ of pressure for the duration of one hour. The powder-particle mixtures were introduced into a graphite die and graphite punch sets were used for hot-pressing. A steady temperature ramp rate of $20^{\circ} \mathrm{C} / \mathrm{min}$ was utilized under an inert argon gas environment of $0.6 \mathrm{~atm}$. The powder was a mixture of $\mathrm{SiC}$ nanopowder along with a minute amount of oxide additives consisting of yttria $\left(\mathrm{Y}_{2} \mathrm{O}_{3}\right)$, alumina $\left(\mathrm{Al}_{2} \mathrm{O}_{3}\right)$, and silica $\left(\mathrm{SiO}_{2}\right)$. The mass ratio (wt\%) of $\mathrm{SiC}: \mathrm{Y}_{2} \mathrm{O}_{3}: \mathrm{Al}_{2} \mathrm{O}_{3}: \mathrm{SiO}_{2}$ in the powder mixture was accurately controlled to $93: 1.75: 3.25: 2$. Using the hot pressing technique discussed earlier, over 40 pellets were fabricated. The hot-pressed specimens then underwent machining to achieve the desired geometries and tolerances. A portion of pellets do not contain any TRISO particles and are fabricated to: i) investigate the hot-pressing behavior of monolithic $\mathrm{SiC}$ and ii) to be irradiated to determine the stability of this type of monolithic $\mathrm{SiC}$ under neutron irradiation.

Microstructure of the pellets revealed a near fully dense $\mathrm{SiC}$ matrix into which the TRISO particles were randomly dispersed (Figure 88). The interface between the TRISO particle and the SiC matrix exhibited good contact where no major voids or evidence of debonding was observed. Close examination of the matrix microstructure showed a uniform $\mathrm{SiC}$ matrix with small volume fraction of isolated oxide phases. The microstructure of the hot-pressed $\mathrm{SiC}$ was comparable to that of Chemical Vapor Deposition (CVD) in the TRISO particle. Transmission electron microscopy was also utilized to characterize the microstructure of the matrix $\mathrm{SiC}$ where presence of oxide phase at the triple points between the grains was observed. A thin $(\sim 1 \mathrm{~nm})$ oxide layer at the grains boundaries also appeared to be present. 

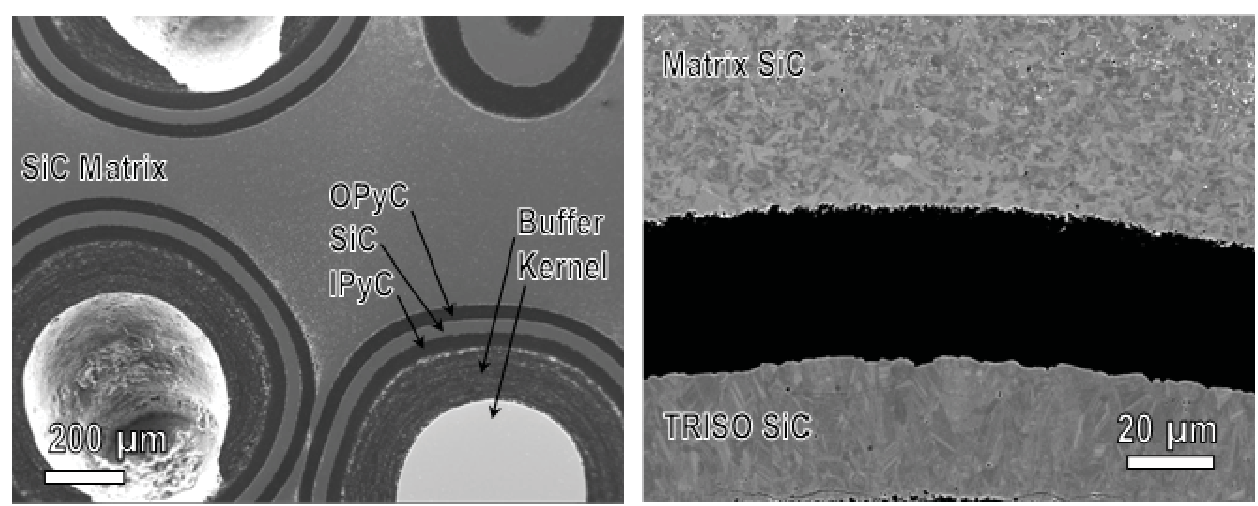

Figure 88. Left: Low-Mag SEM image of TRISI particles embedded in SiC matrix. Right: comparison of grain structure in matrix and particle's CVD-deposited SiC.

Based on the methodology developed above, a series of pellets were fabricated with the diameter of 9 $\mathrm{mm}$, intended for irradiation in flux trap region of HFIR. The pellets were subsequently machined into geometries corresponding to that of oxide pellets in $17 \times 17$ Westinghouse PWR bundle. 20 pellets from this fabricated batch were chosen to be inserted into the reactor. Figure 89 is an example of one of the rabbits where the pellets are shown prior to assembly into the irradiation capsule.

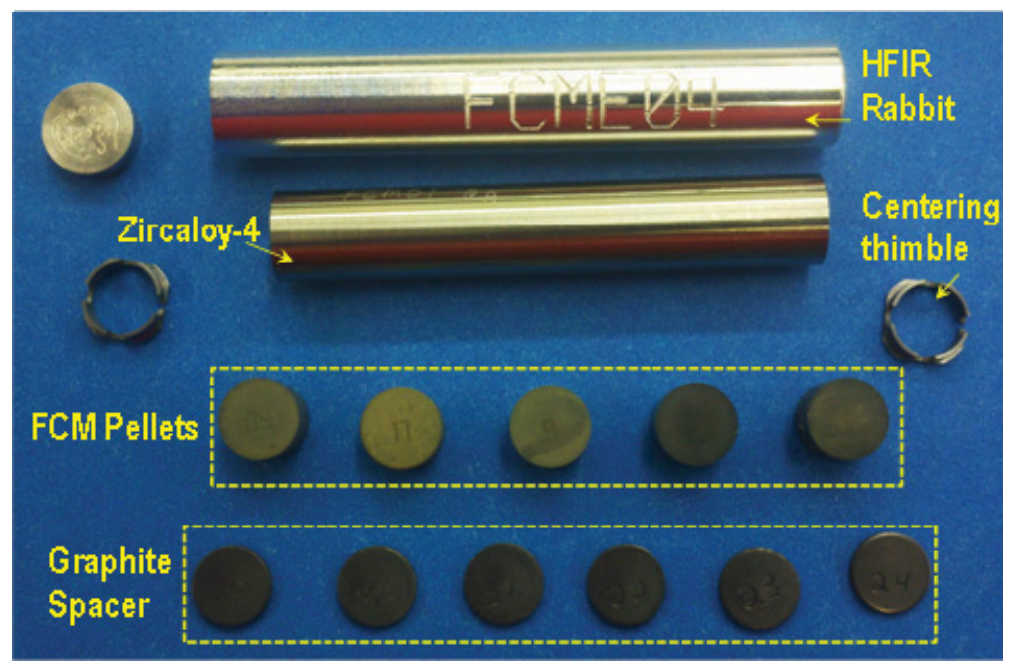

Figure 89. FCM pellets along with the zircaloy-4 tubing section, graphite spacers, and centering thimble prior to encapsulation in the HFIR rabit.

\section{References}

1. L. Snead, et al., J. Nuc. Mater., 371 (2007) 329.

2. L.L. Snead, T.D. Burchell, Y. Katoh, J. Nuc. Mater., 381 (2008) 55.

3. L.L. Snead, T.D. Burchell, J. Nuc. Mater., 224 (1995) 222.

\subsubsection{Accident Testing of Current and Advanced Fuel and Cladding Materials}

Soon after the events at Fukushima NPP an effort at ORNL, sponsored by DOE NE's FCRD program, was undertaken to examine fuel behavior under severe accident conditions. The goal of the program is to examine both current and advanced fuel concepts for LWRs under design basis accident conditions and beyond pertaining to that of a loss of coolant event. While NRC regulations define the conditions for a 
design basis loss-of-collant accident (LOCA), prediction of parameters governing materials behavior under a beyond design basis LOCA proves tedious; this is especially the case when advanced fuel systems other than the current oxide/Zircaloy system is to be considered. Since the inception of the program hitherto, the following basic efforts have been undertaken to address the needs outlined above:

- Testing fuel/cladding materials under NRC-defined design basis LOCA conditions in a bench-top scale with the ultimate aim of re-establishing domestic capability for in-cell testing of irradiated fuels in an integral LOCA test station (similar to the one that previously existed at ANL). Representative results shown in Figure 90a.

- Testing fuel/cladding materials under high-temperature, high-pressure steam and steamhydrogen gas environments for prolonged periods of time. This aims at establishing materials behavior for conditions that could be representative of beyond design basis LOCA events. Representative results shown in Figure 90a.

- Assembling an international panel of experts to discuss and reach upon a consensus on a set of appropriate conditions for testing fuel/cladding materials under a beyond design basis accident LOCA.

Note that the current fuel system (zirconium alloys and oxide fuel pellets) constitutes only a small fraction of materials undergoing examination. Meanwhile multiple classes of steel alloys as well as ceramic materials that could possibly substitute fuel and cladding modules in the fuel system are also undergoing investigation. The purpose of the efforts outlined above has been to provide the basis for an intelligent debate among the experts to discuss various fuel system options. Meanwhile the information provided though these efforts shall empower the scientific community to make decisions on the specific fuel systems and testing techniques that are of merit to understand and mitigate these severe accident conditions.
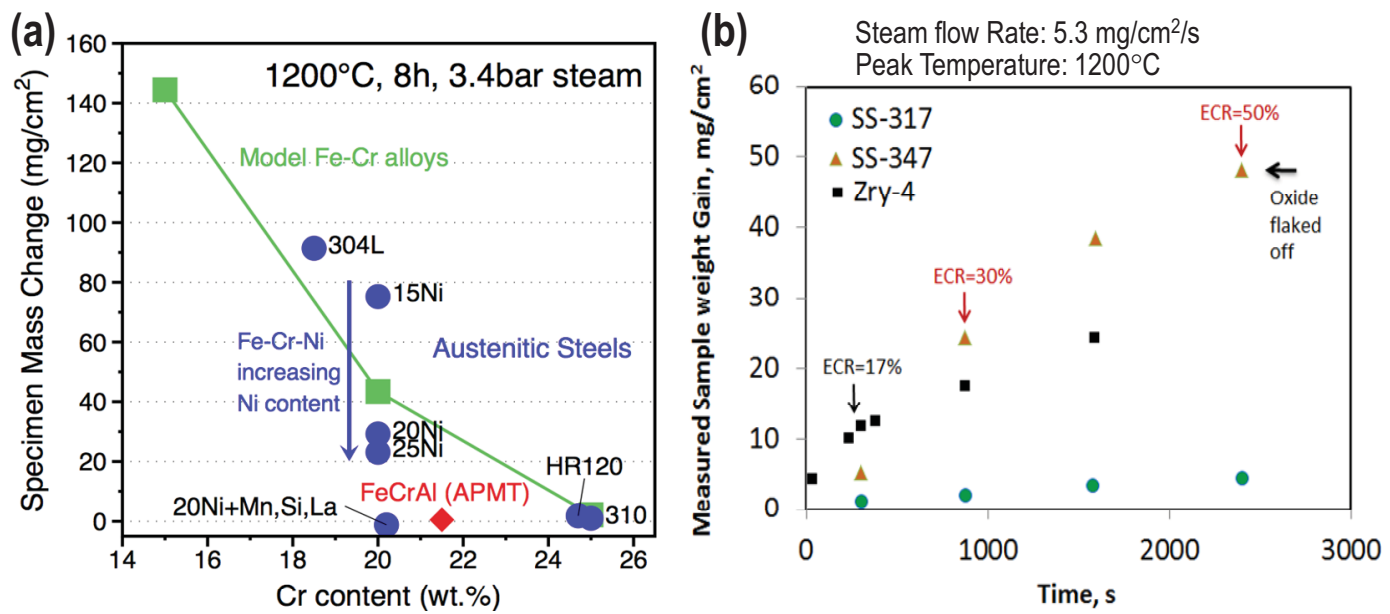

Figure 90. Steam oxidation at a) high-pressure, high-temperature b) atmospheric pressure high flow rate. 


\section{GORE MATERIALS TECHNOLOGIES}

Technical Lead: S. Maloy, maloy@lanl.gov, LANL

Core Materials Technologies focused on fast reactor clad materials for high dose applications in the following R\&D areas: Knowledge base development for High Dose (up to $200 \mathrm{dpa}$ ) Core Materials Irradiation Data (ACO-3 duct testing and Fast Flux Test Facility [FFTF]/Materials Open Test Assembly [MOTA] testing) and Advanced Material Development (advanced cladding materials and coatings/liners to mitigate FCCI).

The analysis of specimens from the ACO-3 Duct involved analysis of Charpy, compact tension, tensile and TEM specimens. A detailed microstructural analysis was performed of the phase distribution along the duct (TEM and APT analysis). Rate jump testing was performed to provide detailed data for model development. Thermal annealing was performed at 550 and $650^{\circ} \mathrm{C}$ followed by fracture toughness testing at ORNL.

For innovative liner development, the design of AFC-3 materials capsule was completed. Modifications were necessary to reach desired temperature. The first cycle capsule will only include cladding-fuel-liner diffusion couple samples. Out of Pile cladding-fuel-liner and coating DC studies are on-going at TAMU.

Coatings were fabricated on HT-9 and MA957 and underwent Fe heavy-ion irradiation at $500^{\circ} \mathrm{C}$. Cross-sections of the coatings were prepared in the FIB and analyzed by a field emission gun-based scanning transmission electron microscope (FEG-STEM).

Innovative processing for oxide dispersion strengthened (ODS) alloys included the fabrication of Friction Consolidation and Extrusion (FCE) dies and the initiation of the experimental work to establish process parameters for densifying powder metal ODS alloy precursors.

A large heat ( $>50 \mathrm{~kg}$ ) of ODS strengthened ferritic steel (14YWT) was produced and bars were consolidated using HIP'ing and hot extrusion followed by forging and rolling processing. Mechanical testing and irradiation testing of this material is underway. First data was obtained on an ODS steel, (MA957) after irradiation in FFTF to doses up to $100 \mathrm{dpa}$. This is the highest dose data reported on this ODS steel.

\subsection{Cladding Development}

\subsubsection{HT-9 Knowledge- based Development to Very High Doses [>200 dpa]}

T. Saleh, tsaleh@lanl.gov, T. Romero, S. Maloy, LANL

A fuel test assembly known as the ACO-3 duct made out of a fully tempered ferritic/martensitic steel (HT-9) was irradiated in the FFTF up to 155 dpa at a temperature range of $380-504^{\circ} \mathrm{C}$. Mechanical testing was performed on samples cut from locations along the length of the duct including tensile testing, Charpy testing and fracture toughness testing. To understand the defects produced from radiation damage at a fundamental level, additional testing has been performed including digital microscopy on the fracture surfaces, strain rate jump testing, thermal annealing tests followed by fracture toughness testing and microstructural analysis. This data will aid in determining the design limits for HT-9, developing atomistic-scale models and investigate methods of using annealing to increase the lifetime.

\section{Digital Optical Microscopy on Tensile Specimens from ACO-3 Duct}

Imaging and measuring of the fracture surfaces of the ACO-3 tensile samples (tested in FY 2010) took place on a Hirox KH-7700 Digital Optical Microscope (DOM) in a hot cell. Fracture surfaces were imaged in both single focus and compilated multifocused images. The 3D multifocused image allows for three dimensional reconstruction of the fracture surface to be made, as well as to provide an easily 
measurable cross section of the fracture surface. Samples were imaged at inclinations of 0,45 and 90 degrees to provide profile and angled views of the fracture surface and surrounding deformation areas. Generally all samples failed in a ductile manner, even the ones with the smallest \% reduction in area or smallest elongation (Sample 6E9-6 $\left(\right.$ Tirr $=380^{\circ} \mathrm{C}$, dose $=22 \mathrm{dpa}$ ) tested at $350 \mathrm{C}$, Figure 91). The fracture surfaces on all the samples measured are extremely clean and shiny, with no degradation in the year since testing. Full image sets and tabulated results can be found in report "Deformation Mechanisms of Irradiated HT-9 Steels from the ACO-3 Duct", M31AF050303.

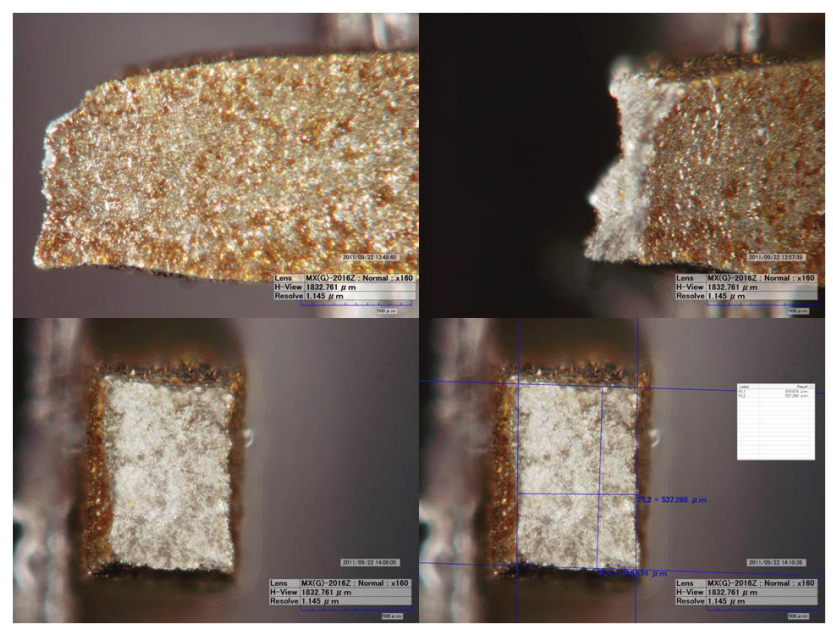

Figure 91. Optical microscopy images of sample $6 E 9-6\left(\left(\right.\right.$ Tirr $=380^{\circ} \mathrm{C}$, dose $\left.=22 \mathrm{dpa}\right)$ at $160 \mathrm{x}$ multiplication. Sample had the lowest uniform, $1.27 \%$, and total elongation, $7.8 \%$, of all samples measured. Ductility is still evident, especially on sample's surface in $45^{\circ}$ image. Clockwise from top left: $2 D$ image at $0^{\circ}, 3 D$ multifocus image at $45^{\circ}, 3 D$ image with measurements at $90^{\circ}, 3 D$ image at $90^{\circ}$.

\section{Rate Jump Experiments}

Rate jump samples were tested on a $30 \mathrm{kN}$ capacity Instron 5567 screw driven load frame located inside a hot cell in Wing 9 at the CMR facility at Los Alamos National Laboratory

Tests were performed at a variable engineering strain rate of $10^{-3}$ and $10^{-4} / \mathrm{sec}$. Initial strain rate was $10^{-4} / \mathrm{sec}$ until near the yield point and then the strain rate was changed roughly every $0.5 \%$ plastic strain. Seven to nine jumps were made for each sample. Load/displacement data were converted to engineering stress/strain data using the initial measured specimen dimensions.

Two rate jump experiments were completed, on control material from the INL lot and an irradiated sample from location 6E5 (Tirr $=398^{\circ} \mathrm{C}$, dose $\left.=42 \mathrm{dpa}\right)$. Results can be seen in Figure 92. 


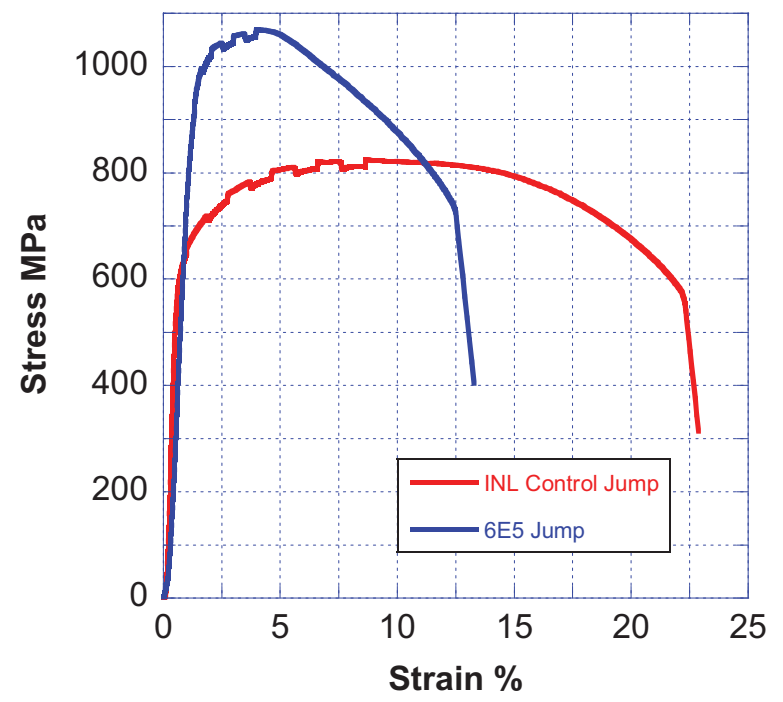

Figure 92. Rate jump experiments for HT-9 INL Control and 6E5 samples

The data in this set will be expanded to include the rest of the irradiation conditions at room temperature. After iteration with modelers a decision will be made to continue with experiments at either higher temperatures or under different strain rate conditions. The data will inform models of rate dependency vs. radiation damage.

\subsubsection{Effect of Thermal Annealing on Fracture Toughness in HT9}

T. S. Byun, byunts@ornl.gov, ORNL

The recovery of fracture toughness by thermal annealing treatment has been studied for the HT9 steel from the ACO-3 duct of FFTF and reported as M31AF050802. The main objective of this study was to suggest possible means for the life extension of core materials by mitigating the effects of long term radiation damage. Three-point bend (TPB) fracture toughness tests have been performed for the irradiated and annealed and non-irradiated HT9 specimens in a servohydraulic testing machine in vacuum condition. The specimens used in this research were the broken halves of Charpy bars $(13 \times 3 \times 4 \mathrm{~mm})$.

Figure 93 displays the effect of $550^{\circ} \mathrm{C}$ annealing on fracture toughness in the irradiated HT9 steel. For the majority of low temperature irradiation cases $\left(<430^{\circ} \mathrm{C}\right)$, the $550^{\circ} \mathrm{C}$ annealing induced only a partial recovery in fracture toughness. Little fracture toughness recovery occurred when the irradiation temperature was about $464^{\circ} \mathrm{C}$ or higher than $430^{\circ} \mathrm{C}$. This indicates the long term irradiation in FFTF has produced significant amount of thermally stable defect clusters such as voids, gas bubbles, and certain types of precipitates. Such a partial or little recovery in fracture toughness is different from the recovery behavior of hardening damage: the microhardness data reported in last year indicated a complete recovery of hardening damage by annealing in the same condition.

Even though the change of fracture toughness by the $550^{\circ} \mathrm{C}$ thermal annealing indicates only a partial or negligible recovery, the thermal annealing treatment is believed to be useful for damage mitigation because the low fracture toughness values obtained after low temperature irradiations show relatively higher degree of recovery. Combining this behavior with the high level of fracture toughness maintained during high temperature irradiation, it can be recognized that the fracture mode after thermal annealing is always ductile and fracture toughness values $(>130 \mathrm{MPa} \sqrt{\mathrm{m}})$ are high enough for maintaining structural stability. 

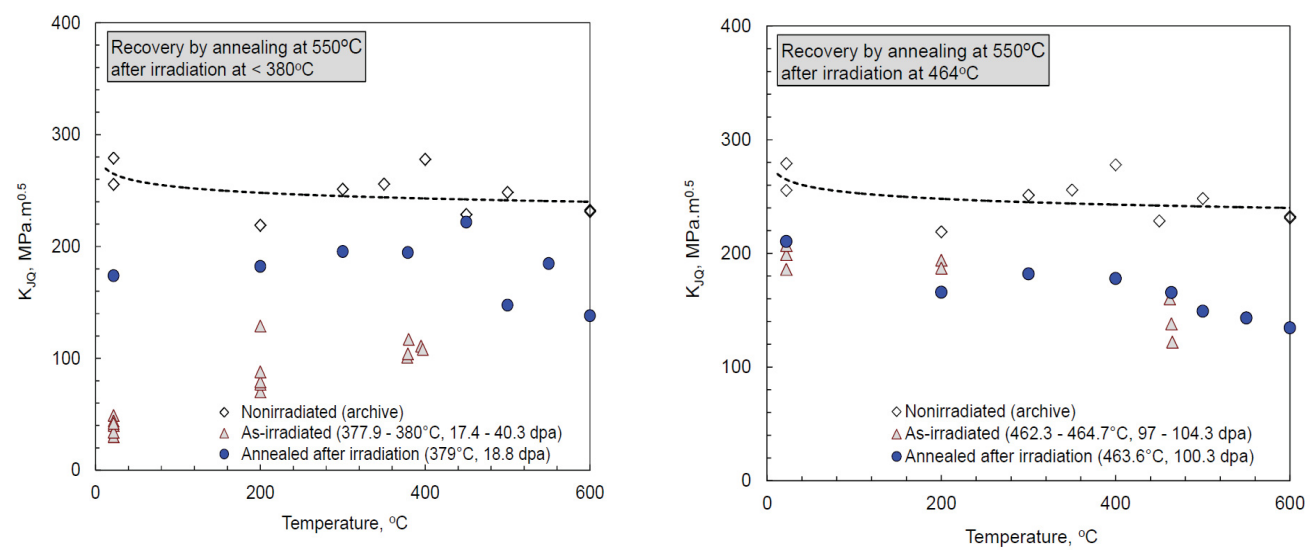

Figure 93. Effect of $550^{\circ} \mathrm{C}$ annealing on fracture toughness in $\mathrm{HT9}$ steel irradiated at about $379^{\circ} \mathrm{C}$ and $464^{\circ} \mathrm{C}$

After the $650^{\circ} \mathrm{C}$ annealing nearly complete or more than $100 \%$ recovery was observed in all fracture tests except for the case tested at $600^{\circ} \mathrm{C}$, as seen in Figure 94. Such a more than complete recovery indicates that the $650^{\circ} \mathrm{C}$ annealing induces additional microstructural changes such as the coarsening of precipitates and/or annihilation of dislocations in addition to the thermal annihilation of small radiationinduced defects. A significant drop in the fracture toughness occurred between 500 and $600^{\circ} \mathrm{C}$ in the test after the $650^{\circ} \mathrm{C}$ annealing. It is notable however that the lowest fracture toughness measured at $600^{\circ} \mathrm{C}$ was still higher than $170 \mathrm{MPa} \sqrt{\mathrm{m}}$.
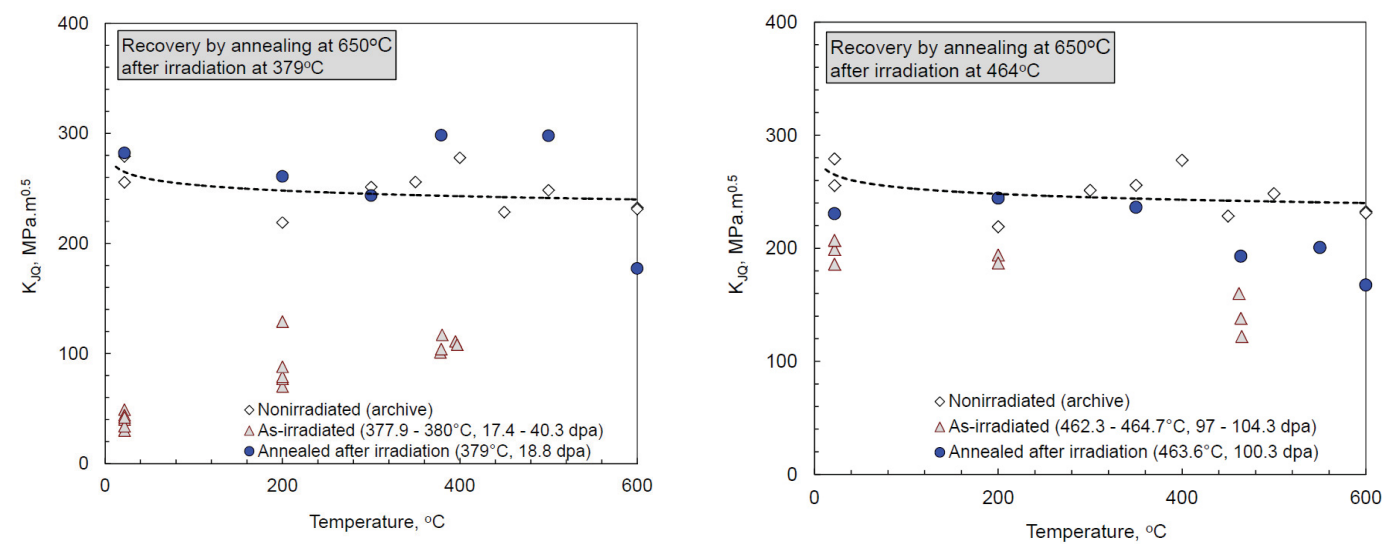

Figure 94. Effect of $650^{\circ} \mathrm{C}$ annealing on fracture toughness in $\mathrm{HT9}$ steel irradiated at about $379^{\circ} \mathrm{C}$ and $464^{\circ} \mathrm{C}$

\subsubsection{Microstructural Analysis of ACO-3 duct:}

O. Anderoglu, J. Van den Bosch- LANL, B. Sencer- INL;

The microstructures of the samples from 5 different zones along the length of the duct were analyzed using a combination of TEM based techniques, APT, small angle neutron scanning (SANS), and X-ray and Neutron diffraction. At moderate irradiation temperatures $\left(\leq 440^{\circ} \mathrm{C}\right)$ a high density of second phase precipitates, $\mathrm{Cr}$ rich $\alpha^{\prime}$ and G-phase were found. The precipitation densities of the second phases are more sensitive to temperature than the dose. Similarly carbide volume fraction increased with increasing temperature while the dislocation density dropped. The second phase particles were found at higher temperatures $\geq 466^{\circ} \mathrm{C}$ despite a high dose of 92 dpa using TEM and APT. However, diffraction studies suggested another phase that was thought to be Chi or $\eta$ phase at higher irradiation temperatures. While 
$\mathrm{Cr}$ was depleted, $\mathrm{Si}, \mathrm{Mn}$, and Ni segregation to grain boundaries were observed. HT-9 showed remarkable resistance to void swelling, $0.3 \%$ at $155 \mathrm{dpa}$ at $440^{\circ} \mathrm{C}$. Void formation was very sensitive to temperature. Voids were detected at $100\left(410^{\circ} \mathrm{C}\right)$ but none were seen at $96 \mathrm{dpa}\left(466^{\circ} \mathrm{C}\right)$ zone. In contrast to what is reported in the literature, no laves phases was found in any of the zones. Details of these analyses can be found in M31AF050301, Report on Microstructural Analysis in the ACO-3 duct.

\subsubsection{High dose Advanced Materials}

Tarik A. Saleh, tsaleh@lanl.gov, Tobias J. Romero, Stuart A. Maloy, LANL

Samples from the STIP-IV (SINQ Target Irradiation Program) irradiation were received at Los Alamos National Laboratory on 8/5/2011. Samples were moved to the hot cells in Wing 9 of CMR on $8 / 10 / 11$ and inspection of the samples and initial testing commenced immediately. The conditions of the STIP-IV irradiation that these samples were subjected to is detailed in the report "A preliminary report of STIP-IV" by Yong Dai of the Paul Scherrer Institut in Switzerland.

After samples were removed unloaded, two HT-9 samples (UG-13 and UG-14) and one sample of 14YWT (FD-02) were selected for testing. Unirradiated control samples for these two materials were tested as a part of this initial testing effort. Additional comparison data is drawn from HT-9 samples tested from the ACO-3 duct, both control and irradiated. Samples tested are listed in Table 9.

Table 9. Tested sample summary

\begin{tabular}{|c|c|c|c|c|}
\hline Sample Name & Material & Irradiation & Dose & Irradiation Temperature \\
\hline UG-13 & HT-9 & STIP-IV & $\sim 20 \mathrm{dpa}$ & TBD \\
\hline UG-14 & HT-9 & STIP-IV & $\sim 20 \mathrm{dpa}$ & TBD \\
\hline Control & HT-9 & STIP_IV Control & NA & NA \\
\hline 6 E 9 & HT-9 & ACO-3 & $21.8 \mathrm{dpa}$ & NA \\
\hline Control & HT-9 & ACO-3 Control & NA & TBD \\
\hline FD-02 & $14-$ YWT & STIP-IV & $\sim 20$ dpa & NA \\
\hline Control & $14-$ YWT & STIP_IV Control & NA & \\
\hline
\end{tabular}

Neither the HT-9 nor the 14YWT samples tested from the STIP-IV irradiation displayed any plasticity. The nonlinearity in the elastic region is believed to be due to the samples slipping in the grips. Initial post test inspection yielded no measurable plasticity at the fracture. There was evidence of some off-axis loading, due to cracks in the grips, possibly causing premature fracture. Yield and ultimate tensile strength (UTS) are believed to be the same for these samples at this point. Further testing and inspection is ongoing, a complete summary can be found in milestone report on First Tensile Tests on Neutron Irradiated Advanced Materials, M21AF050401.

\subsubsection{High Dose FrTF Specimen Testing}

Mychailo B. Toloczko, mychailo.toloczko@pnnl.gov, PNNL

With mechanical testing capabilities reestablished at Pacific Northwest National Laboratory (PNNL), work was able to resume on mechanical property testing of FFTF high dose specimens recovered from storage. There were two main task areas for FY 2011: 1) Baseline tensile testing of irradiated MA957 ODS steel, and 2) Phase 1 fracture toughness testing of irradiated HT-9.

Tensile tests on MA957 were performed on axial tensile specimens cut from pressurized tubes that were recovered from FFTF. The irradiation temperature ranged from as low as $385^{\circ} \mathrm{C}$ to as high as $750^{\circ} \mathrm{C}$ while the dose ranged from $43 \mathrm{dpa}$ to $103 \mathrm{dpa}$. Baseline room temperature tests were performed as shown in Figure 95. The testing went very smoothly with the only unusual result being the response of the specimen that was irradiated at $550^{\circ} \mathrm{C}$. This specimen showed a much higher rate of hardening than both 
the specimen irradiated at a lower temperature of $495^{\circ} \mathrm{C}$ and the specimen irradiated at a higher temperature of $603^{\circ} \mathrm{C}$. With the exclusion of the $550^{\circ} \mathrm{C}$ test, the tensile property plot in Figure $95 \mathrm{~B}$ reveals some surprising properties. First is that the yield and ultimate strength of all the irradiated specimens, including those irradiated at $603^{\circ} \mathrm{C}-750^{\circ} \mathrm{C}$ were higher than the unirradiated material. While the increase in strength for irradiation temperatures of $385^{\circ} \mathrm{C}$ and $495^{\circ} \mathrm{C}$ is not surprising, the microstructure and mechanical properties of ferritic/martensitic steels are typically dominated by thermal aging when irradiated at temperatures above $550^{\circ} \mathrm{C}$ with the result that the strength is typically the same or lower than the unirradiated material due to coarsening of the microstructure. The strength of ODS steels arises primarily from the oxide particle population, and thus the increase in strength suggests that the irradiation is having some effect on the oxide particles. The leading hypothesis is that ballistic dissolution is refining or changing the oxide particle size or number density. The other surprising trend in properties is the high uniform elongation preserved after irradiation. Irradiation and thermal effects on microstructure typically reduce the uniform elongation of irradiated ferritic/martensitic steels. With the exception of the specimen irradiated at $385^{\circ} \mathrm{C}$, the uniform elongation is as good or better than the unirradiated material. As with the surprising strength of the material after irradiation, the high uniform elongation is suggestive of a change in the oxide particle population. Transmission electron microscopy studies will take place in FY 2012 to begin to determine these postirradiation properties of this material.
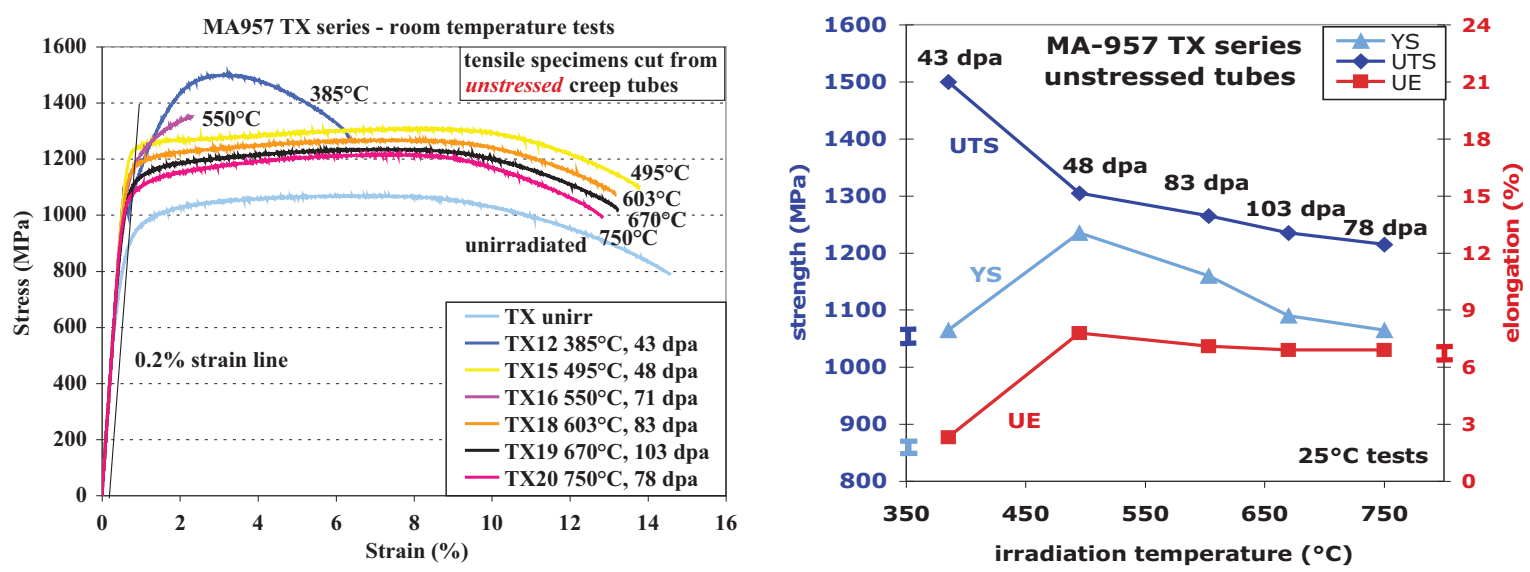

Figure 95. (A) Tensile traces and, (B) tensile property plot for irradiated MA957 ODS steel. Tensile properties of the unirradiated material are indicated by the bold "I" markers on the vertical axes in B).

The other major mechanical property test effort for FY 2011 was fracture toughness testing of irradiated HT-9. Testing focused primarily on testing at room temperature but selected tests were also performed at $200^{\circ} \mathrm{C}$ (prototypic fuel handling temperature) and at the irradiation temperature. Several different heats were tested with the results presented in Figure 96. In general, the results mirrored those that have been performed on the ACO-3 duct with fracture toughness being very low on materials irradiated below $400^{\circ} \mathrm{C}$ and being approximately $30 \%$ lower than unirradiated material at tests above $400^{\circ} \mathrm{C}$. One interesting exception are tests on two different heats at approximately $410^{\circ} \mathrm{C}$. One heat showed very brittle response while the other showed ductile tearing. Further testing and analysis will be performed in FY 2012. 


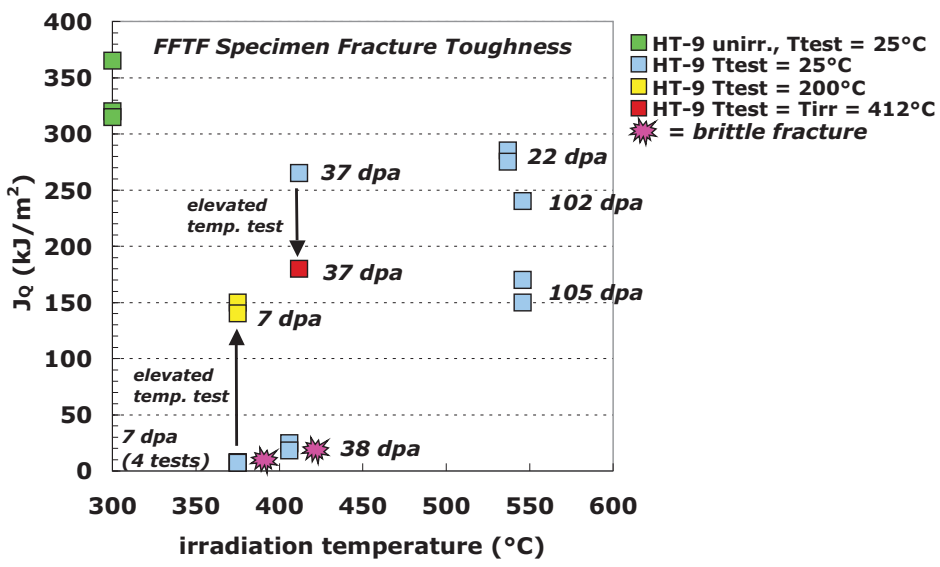

Figure 96. Fracture toughness of irradiated HT-9 specimens recovered from FFTF.

\subsubsection{Ma957 Irradiation Greep and Swelling}

\section{Mychailo B. Toloczko, mychailo.toloczko@pnnl.gov, PNNL}

Irradiation creep and swelling measurements were performed on two heats of MA957 pressurized tube in-reactor creep specimens irradiated in FFTF up to $110 \mathrm{dpa}$ at temperatures from $385^{\circ} \mathrm{C}$ to $750^{\circ} \mathrm{C}$. Swelling was assessed by diameter change measurements of unpressurized tubes and by density change meaurement of sections of both unpressurized and pressurized tubes with the results shown in Figure 97. Swelling estimated from diameter change shows a linear increase as a function of irradiation temperature with a maximum of almost $1 \%$ indicated swelling at $750^{\circ} \mathrm{C}$ while swelling from density measurement shows a flat response of $0.3 \%$ that is independent of both irradiation temperature and stress. Diameter change measurements are sensitive to swelling, precipitation, and texture-induced grain shape change while density measurements are sensitive to only swelling and precipitation. The density data are considered more reliable for swelling measurements and suggest little or no swelling at all irradiation temperatures $(0.3 \%$ is within the error of the technique). The flat response of the density change data also suggests that the increase in diameter as a function of irradiation temperature is likely being driven by shape change of the grains. Since MA957 has a highly elongated grain shape with an 10:1 grain aspect ratio that has the long axis parallel to the tube axis, grain shape change is a reasonable explanation for the increase in diameter as a function of irradiation temperature because the shape change process will be driven more strongly at higher temperatures.

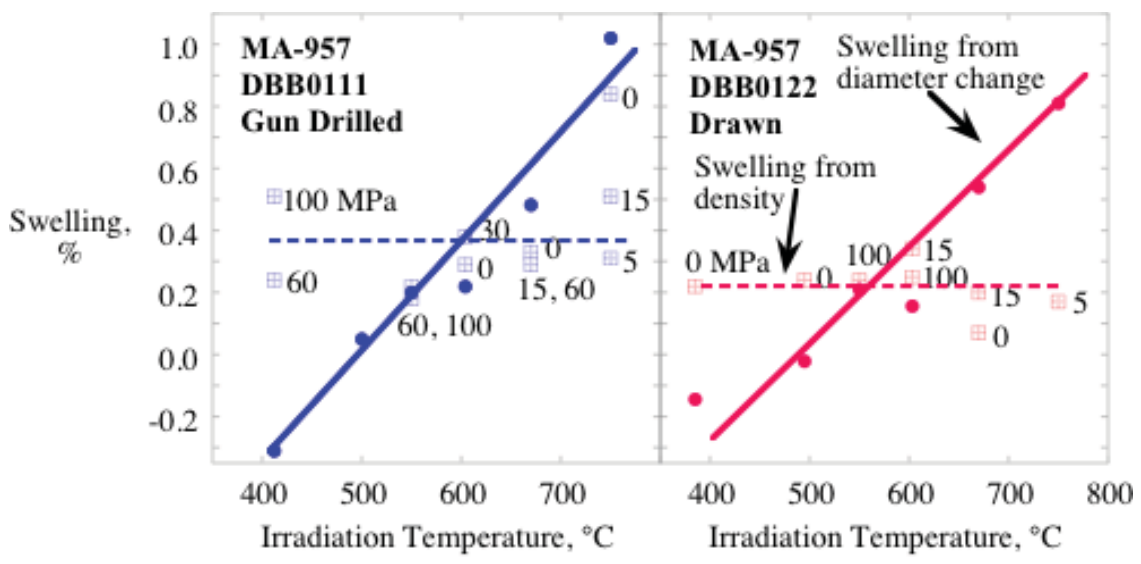

Figure 97. Swelling estimated from diameter change of unpressurized tubes and from density change of pieces of both unpressurized and pressurized tubes. The stress of each tube measured by density is indicated in the plots. 
The steady state in-reactor creep rate was also measured as a function of irradiation temperature and is shown in Figure 98 along with results for HT-9. Both materials have the same steady state creep rate up to $550^{\circ} \mathrm{C}$, and then $\mathrm{HT} 9$ begins to increase rapidly due to thermal creep effects while MA957 is independent of irradiation temperature up to about $600^{\circ} \mathrm{C}$ and then shows a slight upward trend to the peak irradiation temperature of $750^{\circ} \mathrm{C}$. This is the first clear in-reactor demonstration that MA957 has superior creep resistance over a traditional ferritic/martensitic steel. Another valuable conclusion from the data is that the in-reactor steady state creep response of ferritic/martensitic steels is apparently independent of microstructure. The high density of nano-sized oxide particles and ultrasmall grain size of MA957 is a much different microstructure than the coarse carbide precipitate population and relatively large lath grains in HT-9. Microstructural observations will begin in FY 2012 to confirm these results.

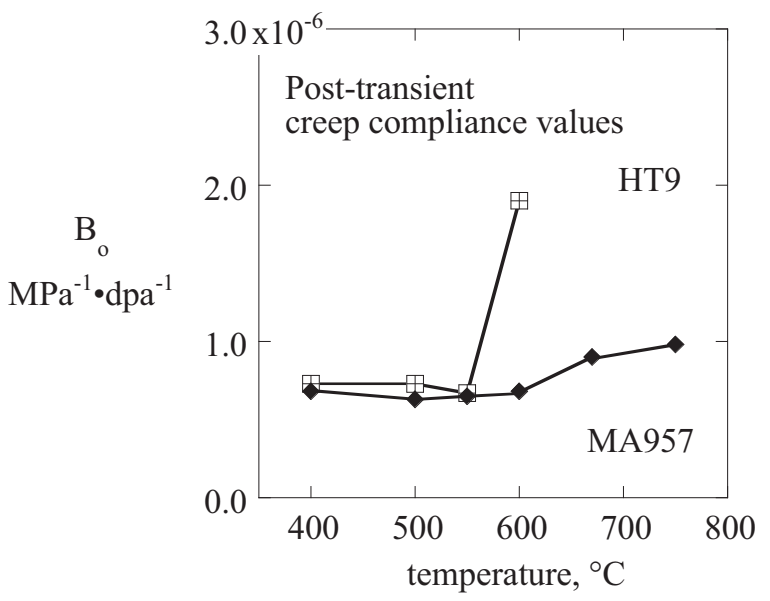

Figure 98. Steady state creep rate of MA957 and HT-9 as a function of irradiation temperature.

\subsubsection{Coating and Liner Development for FCCI Mitigation}

J. Cole, james.cole@inl.gov, J. Gan, Randy Fielding INL, Haiyan Wang, TAMU

The advanced fuel cladding development effort at the INL has focused on fabrication development, characterization and testing of barrier coatings and liners to mitigate or eliminate chemical interactions between nuclear fuel and cladding alloys. Activities during FY 2011 included completion of an HT-9 cladding tube fabrication trial, the conduct of a series of cladding/liner/coating/surrogate fuel interaction studies, the fabrication of $\mathrm{Zr}$-lined cladding tubes for in-reactor testing and the design of an ATR irradiation test to evaluate in-pile liner behavior. The following briefly highlights these efforts.

A fabrication trial to evaluate the capability, within the United States, to produce HT-9 fast reactor cladding tubes that meet stringent NQA-1 quality standards was completed. The effort began in early 2008 and the cladding tubes were delivered late in the summer of 2010 with a summary report being issued in spring of 2011. Fast reactor cladding tubes have not been manufactured in the United States for more than a decade. At the initiation of the fabrication trial in 2008 it was viewed as important to revive this capability for near term fast-reactor deployment activities before the manufacturing knowledge was completely lost. Ultimately, several issues were encountered during fabrication of the tubing, and a significant fraction of the supplied tubing did not meet the required specification. Cladding tubes out of specification will be used for on-going research efforts not involving reactor testing.

Efforts continued during the year to develop effective barrier layers for FCCI mitigation whether solid-liner or coating based options. Zirconium-lined HT-9 cladding tubes were successfully fabricated at Century tubing in San Diego, CA. To evaluate the effectiveness of the barriers, and guide in establishing optimized fabrication parameters for both the liners and the coatings, interaction studies using diffusion couple samples were conducted. Results from the studies suggest $\mathrm{Zr}$ is more compatible with HT-9 than 
$\mathrm{V}$ and that thin coating layers of $\mathrm{TiN}(<500 \mathrm{~nm})$ slow down but do not completely eliminate reaction of Ce with the cladding material. Testing will continue to better understand the factors that influence interdiffusion behavior within the composite fuel-cladding system.

Understanding how potential liner and coating materials behave at temperature under irradiation is the next key step in the process of the fuel-cladding barrier development. To this end, an irradiation experiment was designed to insert into the ATR as part of the AFC-3 experiment. The materials and sample geometry were defined, the thermal design was performed, and capsule design was completed (see the schematics in Figure 99). Due to inadequate thermal losses, the original design temperature was not achievable and therefore the experiment was redesigned with a planned insertion during the 2nd or 3rd cycle of the AFC-3 experiment.
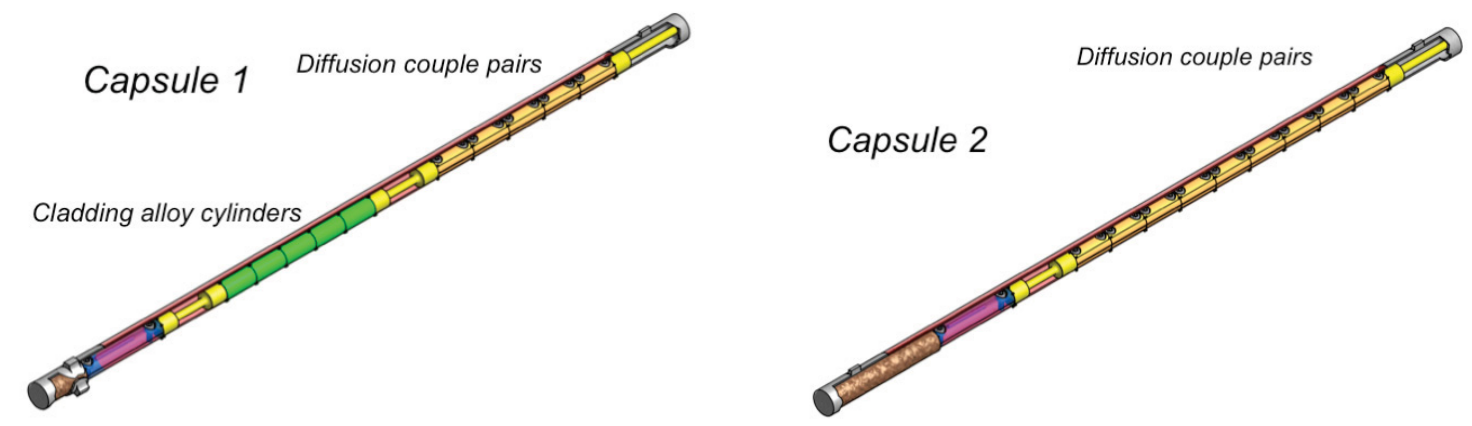

Figure 99. Schematic drawings of AFC-3 materials capsules.

\title{
7.1.8 Innovative Coating Development
}

\author{
J. Cole, james.cole@inl.gov, J. Gan, INL, H.Wang, TAMU
}

A strong and robust ceramic coating can be introduced between the fuel and cladding inner wall to act as an effective diffusion barrier and mechanical buffer to either completely stop or significantly reduce the chemical interaction and avoid the stress buildup at the cladding due to the various fuel-clad interaction induced changes. The radiation response of the nano-scale TiN and TiN/AlN multilayer coatings (500-1000 $\mathrm{nm}$ thickness) and the microstructural development at the coating/cladding interface to high doses ( $\sim 200 \mathrm{dpa})$ under irradiation temperatures relevant to fast reactor cladding $\left(500-600^{\circ} \mathrm{C}\right)$ are not known. As an initial study to evaluate high-dose behavior of innovative ceramic coatings on an HT-9 or ODS substrates, Fe heavy-ion irradiations were conducted at the Michigan Ion Beam Facility in the summer of FY 2010. The dose levels achieved during this experiment were 10, 50 and $200 \mathrm{dpa}$. Using a FIB system, cross-section TEM samples of the heavy ion irradiated materials were prepared and the samples were analyzed in the FEG-STEM during FY 2011. The samples irradiated included the following:

1. HT9, TiN coating, deposited at room temperature.

2. HT9, TiN coating, deposited at $500^{\circ} \mathrm{C}$.

3. HT9, TiN/AlN coating, deposited at $500^{\circ} \mathrm{C}$.

4. MA957, TiN coating, deposited at room temperature.

5. MA957, TiN coating, deposited at $500^{\circ} \mathrm{C}$.

6. MA957, TiN/AlN coating, deposited at $500^{\circ} \mathrm{C}$.

Images of a TiN/TaN multilayer coating on HT-9 irradiated to 10 dpa are shown in Figure 100. The analyses revealed significant stability of the multilayer coating at elevated temperature to a dose of 10 dpa. The stability is evident because no damage from heavy-ion bombardment is observed. Chemical 
analysis across the layer interfaces also indicates little mixing. These investigations are on-going and will lead to a better understanding of coating behavior under irradiation.

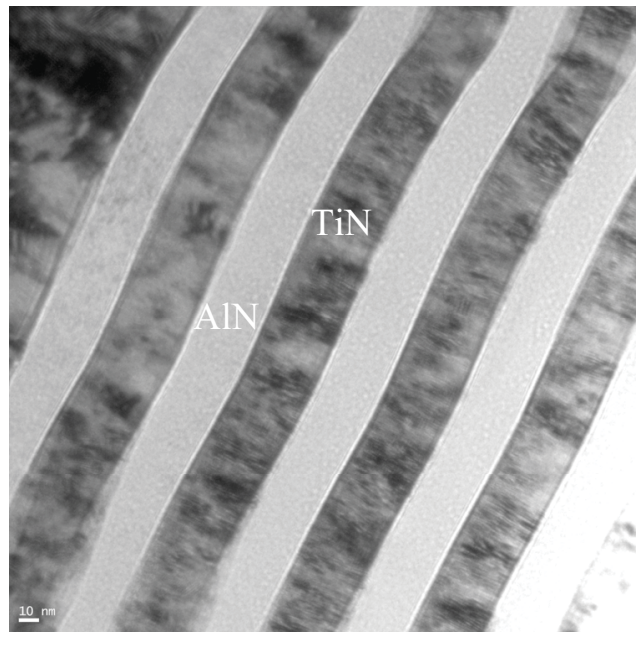

(a)

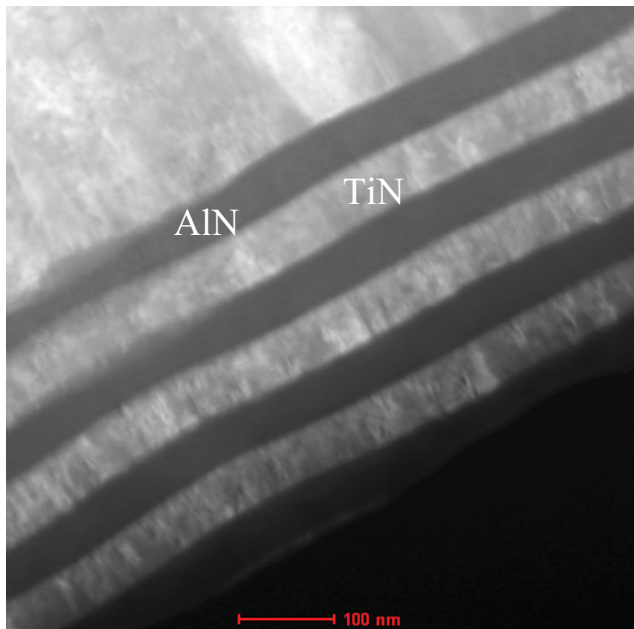

(b)

Figure 100. (a) TEM image showing minimal evidence of radiation damage in coating layers after 10 dpa at $500^{\circ} \mathrm{C}$; (b) high angle annular dark field STEM image illustrating compositional variation in coating layers and little evidence of elemental mixing.

\subsection{Advanced Clad Materials Development}

\subsubsection{Development of Large Scale Heat of an Advanced ODS Alloy}

D. Hoelzer, ORNL, hoelzerd@ornl.gov, S. Maloy, D. Hammon, LANL, G.R. Odette, UC-Santa Barbara, B.D. Wirth, University of Tennessee-Knoxville (formerly UC-Berkeley), P. Hosemann UC-Berkeley (formerly LANL), M. West, South Dakota School of Mining and Technology

Several large heats of the advanced ODS 14YWT ferritic alloy were produced to investigate the effects of processing parameters and fabrication methods on the mechanical properties. The primary purpose of the investigation on processing parameters was to obtain an understanding of how to reduce the contamination of gas atomized Fe pre-alloyed powders by $\mathrm{O}, \mathrm{C}$, and $\mathrm{N}$ interstitial atoms during ball milling. The purpose of investigating fabrication methods was to determine whether the anisotropy introduced by mechanical alloying processing can be reduced. Both controlling the $\mathrm{O}, \mathrm{C}$ and $\mathrm{N}$ levels in the ball milled powders and reducing anisotropy are key factors that will be used to produce the large 55 $\mathrm{kg}$ heat of 14YWT involving ball milling the powder at the pilot facility operated by Zoz, GmbH, Germany and subsequent consolidation by extrusion and hot isostatic pressing (HIP) during the current fiscal year.

\subsubsection{Advances in Processing}

\section{D.T. Hoelzer, hoelzerd@ornl.gov, ORNL}

Four heats of the advanced ODS 14YWT ferritic alloy were produced with the larger capacity Zoz CM08 attritor mill located at ORNL. The $3.0 \mathrm{~kg}$ ( $6.6 \mathrm{lbs}$.) 14YWT-SM11 heat was produced first using the typical ball milling parameters. The chemical analysis results for this heat showed that significant contamination of $\mathrm{O}$ and $\mathrm{N}$ occurred during ball milling of the powder as shown in Table 10 . The $2.4 \mathrm{~kg}$ ( $\sim 5.3$ lbs.) 14YWT-PM1 heat was the next large heat that was produced, but was the first heat produced using the modified ball milling condition. The chemical analysis results for 14YWT-PM1 showed that the modified ball milling condition successfully reduced the contamination level of $\mathrm{O}, \mathrm{C}$ and $\mathrm{N}$, as shown in Table 10. However, the Fe pre-alloyed powder (L2311 heat) used for producing this heat had been gas 
atomized to contain $0.2 \% \mathrm{Y}$ and a low $\mathrm{O}$ content of $0.014 \%$, which was significantly too low to produce the desired high number density of nano-size oxide particles typical of the previous heats of 14YWT. The $3.0 \mathrm{~kg}$ ( $\sim 6.6 \mathrm{lbs}$.$) 14YWT-SM12 heat was the next experimental heat that was produced using the$ modified ball milling condition. In this case the Fe pre-alloyed powder was ball milled with $0.3 \% \mathrm{Y}_{2} \mathrm{O}_{3}$ powder to elevate the O level. The chemical analysis of the 14YWT-SM12 heat showed that the O content could be controlled to the desired level while maintaining lower $\mathrm{C}$ and $\mathrm{N}$ levels during ball milling as shown in Table 10 . The $1.0 \mathrm{~kg}(\sim 2.2 \mathrm{lbs}$.) 14YWT-PM2 heat was produced with powders that had a similar composition as the large $55 \mathrm{~kg}$ heat that will be used for scale-up production of the advanced ODS 14YWT ferritic alloy. In this heat, the O content was increased to the desired level by ball milling $\mathrm{FeO}$ powder with the Fe-pre-alloyed $\mathrm{L} 2311$ powder that contained $0.2 \% \mathrm{Y}$ and $0.014 \% \mathrm{O}$. The chemical analysis shown in Table 10 demonstrated that this experimental procedure was successful in obtaining a high $\mathrm{O}$ level of 1352 wppm while keeping the $\mathrm{C}$ and $\mathrm{N}$ levels at a low value of 140 wppm, respectively. Figure 101 shows a bar graph depicting the total amount of $\mathrm{O}, \mathrm{C}$ and $\mathrm{N}$ picked up during the production of the four large heats of 14YWT.

Table 10. Chemical analysis results of ball milled powder and extruded heats of 14YWT.

\begin{tabular}{|c|c|c|c|c|c|c|c|c|}
\hline \multirow{3}{*}{$\begin{array}{l}\text { Element } \\
\text { (wppm) }\end{array}$} & \multirow{2}{*}{\multicolumn{2}{|c|}{$\begin{array}{c}\text { 14YWT-SM11 } \\
\text { Fe-14Cr-3W-0.4Ti + } \\
0.3 \% \mathrm{Y}_{2} \mathrm{O}_{3}\end{array}$}} & \multirow{2}{*}{\multicolumn{2}{|c|}{$\begin{array}{c}\text { 14YWT-P+M1 } \\
\text { L2311 (Fe-14Cr-3W- } \\
0.4 \text { Ti-0.20Y-0.014O) }\end{array}$}} & \multirow{2}{*}{\multicolumn{2}{|c|}{$\begin{array}{c}\text { 14YWT-SM12 } \\
\text { Fe-14Cr-3W-0.4Ti + } \\
0.3 \% \mathrm{Y}_{2} \mathrm{O}_{3}\end{array}$}} & \multicolumn{2}{|c|}{ 14YWT-PM2 } \\
\hline & & & & & & & $\mathbf{L} 2311+0$ & $\% \mathrm{FeO}$ \\
\hline & Atomized & Milled & Atomized & Milled & Atomized & Milled & Atomized & Milled \\
\hline O & $\mathrm{d}$ & 2590 & 140 & 447 & 150 & 1184 & 140 & 1352 \\
\hline $\mathrm{C}$ & 100 & 346 & 60 & 287 & 100 & 128 & 60 & 140 \\
\hline $\mathbf{N}$ & 50 & 1911 & 25 & 123 & 50 & 117 & 25 & 140 \\
\hline
\end{tabular}

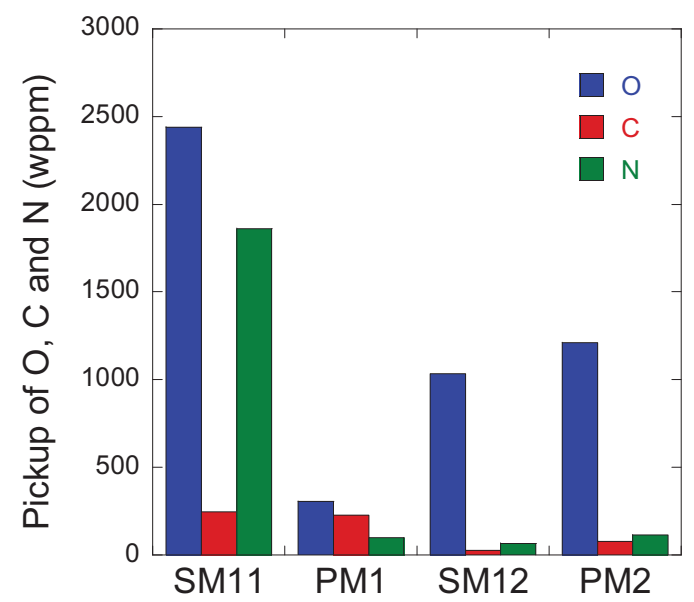

Figure 101. Shows the beneficial effect of using the modified ball milling condition to control the $O, C$ and $N$ levels in the four large heats of 14YWT.

\subsubsection{Advances in Fabrication}

D.T. Hoelzer, hoelzerd@ornl.gov, M.A. Sokolov, sokolovm@ornl.gov, ORNL,

G.R. Odette, odette@engineering.ucsb.edu, UCSB

The fabrication studies consisted of hot rolling extruded material of the 14YWT-SM11 and 14YWTPM2 heats and conducting fracture toughness testing to investigate the effects of controlling the $\mathrm{O}, \mathrm{C}$ and $\mathrm{N}$ levels in the heats and fabricating by cross rolling on reducing the anisotropy characteristic of ODS alloys including 14YWT. The chemical analysis results showed that 14YWT-SM11 contained a high 
concentration of $\mathrm{O}, \mathrm{C}$ and $\mathrm{N}$ (Table 1) while the modified ball milling conditions used to produce 14YWT-PM2 successfully kept the $\mathrm{O}$ level high but lowered the $\mathrm{C}$ and $\mathrm{N}$ levels as desired. In the fabrication study, $14 \mathrm{YWT}$-SM11 was hot rolled parallel to the extrusion direction at $1000^{\circ} \mathrm{C}$ to $40 \%$ reduction in thickness in contrast to 14YWT-PM2, which was hot rolled normal (crossed rolled) to the extrusion direction at $1000^{\circ} \mathrm{C}$ to $50 \%$ reduction in thickness. The fracture toughness tests conducted on 14YWT-SM1 1 were performed at ORNL while those on 14YWT-PM2 were performed at University of California-Santa Barbara (UCSB).

The fracture toughness of both 14YWT heats was examined in two orientations, the L-T and T-L. It is well known now that fracture toughness of ODS alloys produced by mechanical alloying have very strong orientation dependence. For specimens of L-T orientation, the tension is applied in longitudinal, extrusion (L) orientation, while crack propagates in transverse (T) orientation. Typically, this is the toughest orientation. For specimens in T-L orientation, the tension is applied in T orientation and crack propagates in L orientation. Typically, the T-L orientation is the weakest, from fracture toughness point of view, orientation. Figure 102 illustrates the fracture toughness of the 14YWT-SM11 and 14YWT-PM2 heats in the two orientations. The results showed that the fracture toughness transition temperature, $\mathrm{T}_{0}$, for 14YWT-SM11 was $48^{\circ} \mathrm{C}$ in the L-T orientation and increased by $63^{\circ} \mathrm{C}$ to $111^{\circ} \mathrm{C}$ in the T-L orientation. However, recent results obtained for 14YWT-PM2 showed that $\mathrm{T}_{\mathrm{o}}$ was considerably lower $\left(\sim-150^{\circ} \mathrm{C}\right)$ than that observed for 14YWT-SM11 for the L-T orientation and that there was no apparent change in $\mathrm{T}_{\mathrm{o}}$ for the T-L orientation. These results are very promising and show that controlling the $\mathrm{O}$ content while lowering the contamination levels of $\mathrm{C}$ and $\mathrm{N}$ during mechanical alloying and applying fabrication procedures that include cross rolling will be key to producing the large scale-up $\sim 55 \mathrm{~kg} 14 \mathrm{YWT}$ heat with excellent mechanical properties.

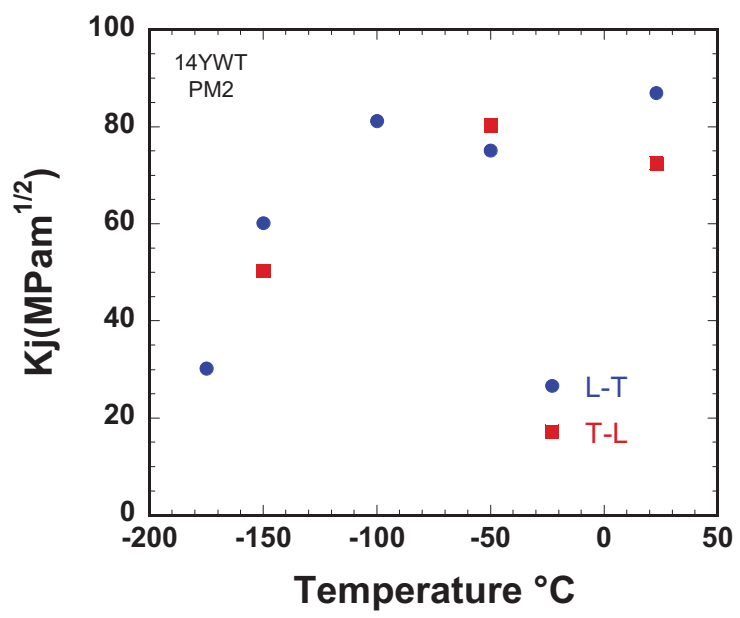

(a)

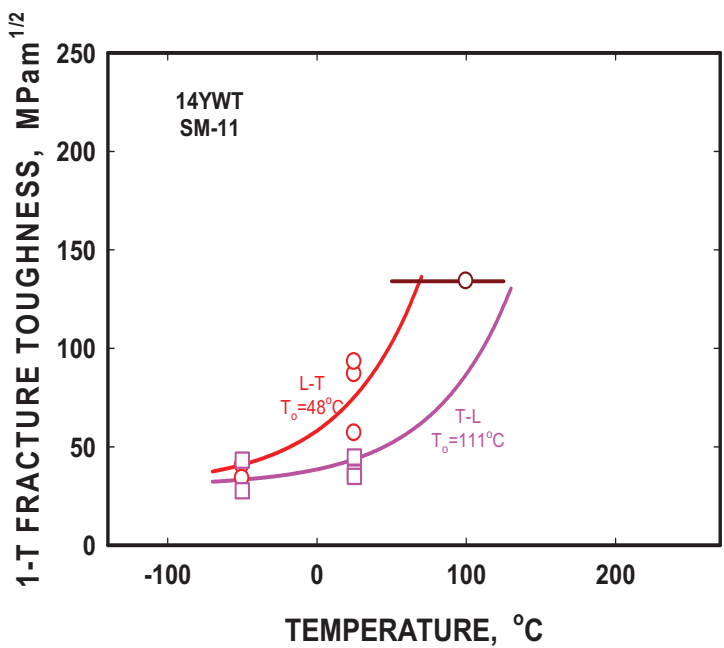

(b)

Figure 102. Fracture toughness of the (a) 14YWT-SM11 heat and (b) 14YWT-PM2 heat in the L-T and TL orientations.

\subsubsection{High Temperature Mechanical Characterization of ODS Alloys}

\section{T. S. Byun, byunts@ornl.gov, ORNL}

The ODS alloys, including the nanostructured ferritic alloys (NFAs) as finer microstructure versions, have been developed as core structural materials for future high temperature reactors. In the past decade, the majority of research efforts were focused on the improvement of high temperature strength, creep strength, and radiation resistance as well as on understanding fundamental phenomena such as nanocluster formation and responses to irradiation. The engineering aspects of NFAs have been largely 
ignored although a high mechanical and structural performance is critical for the applications in extreme conditions. High temperature tensile and fracture tests have been performed for a series of NFAs and reference ODS and ferritic-martensitic (FM) steels to produce basic engineering data for a comparative study and to provide a feedback to the materials development efforts.

Table 11 lists IDs, chemistry and descriptions for test materials. The tensile specimens were the SS-3 type (nominal gage section dimensions: $0.76 \times 7.62 \times 1.52 \mathrm{~mm}$ ) and fracture specimens were bend bars $(2.5 \times 5 \times 15 \mathrm{~mm})$ with $10 \%$ side groove on both sides or $3 \mathrm{~mm}$ thick disk compact tension (DCT) specimens with a diameter of $12.5 \mathrm{~mm}$ and $10 \%$ side grooves. The test temperature ranges were $22-$ $800^{\circ} \mathrm{C}$ for tensile testing and $22-700^{\circ} \mathrm{C}$ for fracture testing.

Table 11. Test materials for uniaxial tensile and fracture toughness tests. 14YWTs and LAFs are in the NFA category; ODS Eurofer 97 and PM2000 have relatively coarse microstructures; NF616 and HT9 steels are non-ODS ferritic and/or martensitic steels.

\begin{tabular}{|c|c|c|}
\hline Material I.D. & Chemistry (wt.\%) & Final TMT \\
\hline 14YWT-SM10 & $\mathrm{Fe}-14 \mathrm{Cr}-3 \mathrm{~W}-0.4 \mathrm{Ti}-0.25 \mathrm{Y}_{2} \mathrm{O}_{3}$ & $\begin{array}{l}\text { Extruded at } 850^{\circ} \mathrm{C} \text {, annealed at } 1000^{\circ} \mathrm{C} \text {, hotrolled at } \\
850^{\circ} \mathrm{C} \text { for } 40 \% \text { reduction. }\end{array}$ \\
\hline 14YWT-SM11CR & $\mathrm{Fe}-14 \mathrm{Cr}-3 \mathrm{~W}-0.4 \mathrm{Ti}-0.25 \mathrm{Y}_{2} \mathrm{O}_{3}$ & $\begin{array}{l}\text { Extruded at } 850^{\circ} \mathrm{C} \text {, hotrolled at } 1000^{\circ} \mathrm{C} \text { for } 40 \% \\
\text { reduction plus cross rolled for } 60 \% \text { reduction. }\end{array}$ \\
\hline 14YWT-SM1HR & Fe- $14 \mathrm{Cr}-3 \mathrm{~W}-0.4 \mathrm{Ti}-0.25 \mathrm{Y}_{2} \mathrm{O}_{3}$ & 13 path hotrolled at $1050^{\circ} \mathrm{C}$ for $\sim 70 \%$ reduction. \\
\hline LAF3C & $\begin{array}{l}\text { Fe- } 8.6 \mathrm{Cr}-2.0 \mathrm{~W}-0.44 \mathrm{Mn}-0.24 \mathrm{Si}-0.29 \mathrm{~V}- \\
0.065 \mathrm{C}-0.08 \mathrm{Ta}-1.0\left(\mathrm{Y}_{2} \mathrm{O}_{3}+\mathrm{TiO}_{2}\right)\end{array}$ & $\begin{array}{l}\text { 1:1 oxide ratio, } 16: 1 \text { reduction extrusion, } \\
1050^{\circ} \mathrm{C} / 1 \mathrm{~h}+\mathrm{AC} \& 750^{\circ} \mathrm{C} / 1 \mathrm{~h}+\mathrm{AC} \text { (Romanoski) }\end{array}$ \\
\hline LAF20 & $\begin{array}{c}\text { Fe- } 8.6 \mathrm{Cr}-2.0 \mathrm{~W}-0.44 \mathrm{Mn}-0.24 \mathrm{Si}-0.29 \mathrm{~V}- \\
0.065 \mathrm{C}-0.08 \mathrm{Ta}-1.0\left(\mathrm{Y}_{2} \mathrm{O}_{3}+\mathrm{TiO}_{2}\right)\end{array}$ & $\begin{array}{l}\text { 2:1 oxide ratio, } 16: 1 \text { reduction extrusion, } \\
1050^{\circ} \mathrm{C} / 1 \mathrm{~h}+\mathrm{AC} \& 750^{\circ} \mathrm{C} / 1 \mathrm{~h}+\mathrm{AC} \text { (Romanoski) }\end{array}$ \\
\hline ODS Eurofer 97 & $\begin{array}{l}\mathrm{Fe}-8.9 \mathrm{Cr}-1.1 \mathrm{~W}-0.2 \mathrm{~V}-0.14 \mathrm{Ta}- \\
0.12 \mathrm{C}+\mathrm{Y}_{2} \mathrm{O}_{3} \text { (typical) }\end{array}$ & Plate \\
\hline PM2000 & Fe-20Cr-5.5Al-0.5Ti- $0.5 \mathrm{Y}_{2} \mathrm{O}_{3}$ (typical) & Block \\
\hline NF616 & $\begin{array}{c}\text { Fe- } 9 \mathrm{Cr}-1.9 \mathrm{~W}-0.45 \mathrm{Mn}-0.45 \mathrm{Mo}-0.2 \mathrm{Ni}- \\
0.1 \mathrm{Nb}-0.2 \mathrm{Si}-0.1 \mathrm{C}-0.05 \mathrm{Cu}-0.011 \mathrm{~S}\end{array}$ & $1079^{\circ} \mathrm{C} / 0.5 \mathrm{~h}+\mathrm{AC} \& 746^{\circ} \mathrm{C} / 1 \mathrm{~h}+\mathrm{AC}(\mathrm{Tan})$ \\
\hline $\begin{array}{l}\text { NF616 mod. } \\
(1539 S T)\end{array}$ & $\begin{array}{c}\text { Fe-9Cr-1.06W-0.5Mn-0.01Mo-0.14Ta- } \\
0.25 \mathrm{Si}-0.32 \mathrm{~V}-0.109 \mathrm{C}-0.055 \mathrm{~N}\end{array}$ & $1070^{\circ} \mathrm{C} / 0.75 \mathrm{~h}+\mathrm{AC} \& 760^{\circ} \mathrm{C} / 0.75 \mathrm{~h}+\mathrm{AC}(\mathrm{Tan})$ \\
\hline HT9 & $\begin{array}{l}\text { Fe-11.9Cr-1.0Mo- } 0.6 \mathrm{Mn}-0.55 \mathrm{~W}-0.5 \mathrm{Ni}- \\
0.3 \mathrm{~V}-0.3 \mathrm{Si}-0.2 \mathrm{C}\end{array}$ & $1030^{\circ} \mathrm{C} / 0.5 \mathrm{~h}+\mathrm{AC} \& 760^{\circ} \mathrm{C} / 1 \mathrm{~h}+\mathrm{AC}$ \\
\hline
\end{tabular}

Figure 103 and Figure 104 present the tensile yield strength and total elongation of the test materials as functions of temperature. The test results for some highly strengthened alloys indicate the strength of NFAs can reach above $2 \mathrm{GPa}$. The most important observation in the comparison of strengths is that the NFAs can retain strengths higher than $500 \mathrm{MPa}$ at a target application temperature of $700^{\circ} \mathrm{C}$, while the strengths of FM steels (NF616s and HT9) are in the range of 320- $430 \mathrm{MPa}$. The strength of traditional ODS steels, ODS Eurofer 97 and PM 2000, do not have clear advantage in strength at very high temperatures. 


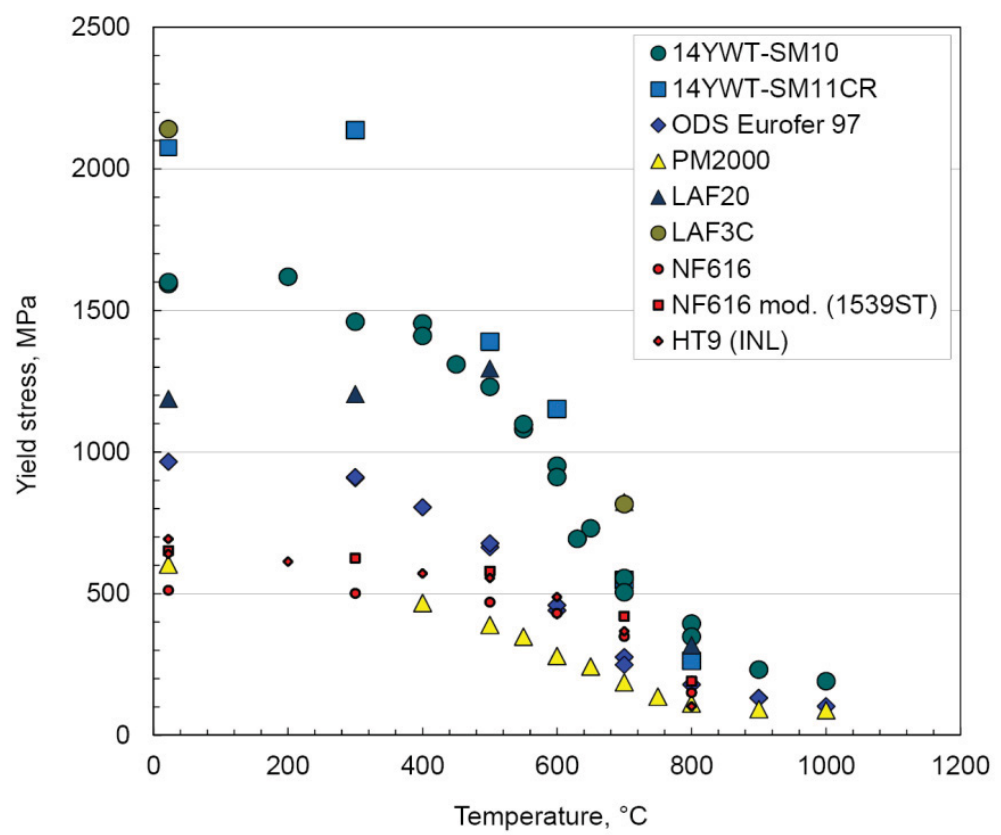

Figure 103. Temperature dependence of tensile yield stress in ODS alloys and reference FM steels.

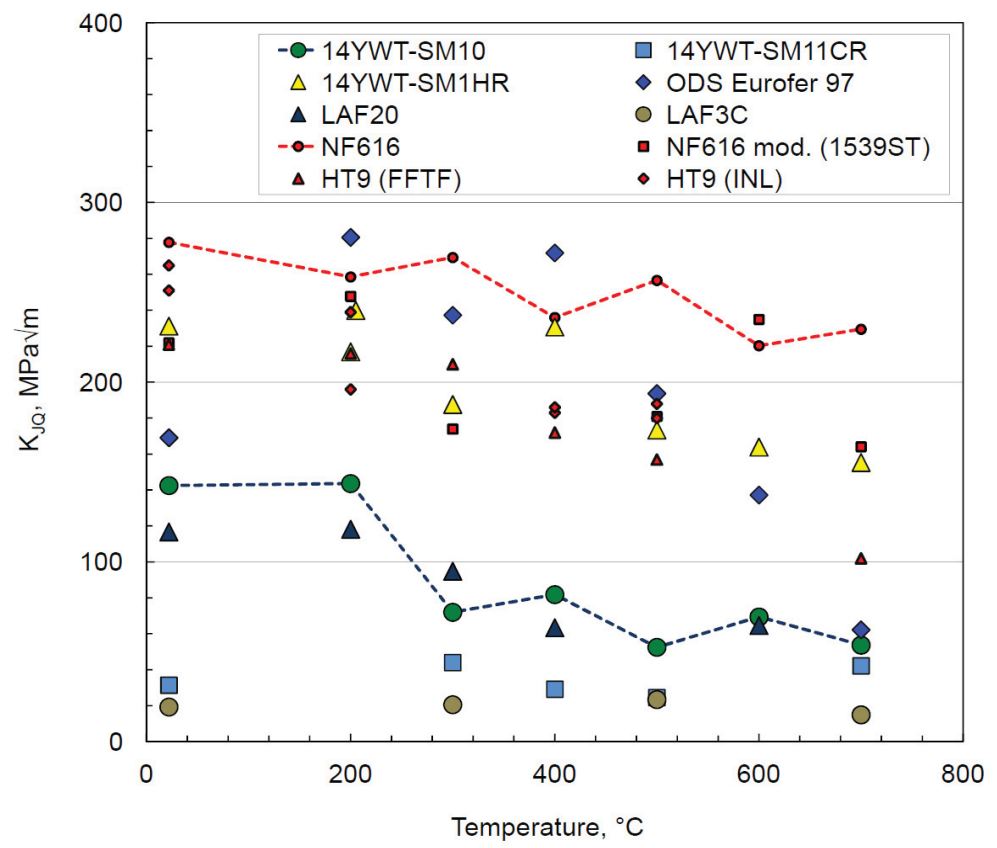

Figure 104. Temperature dependence of fracture toughness in ODS alloys and reference FM steels.

Fracture toughness is considered very important in engineering structure since it characterizes the general resistance of the material to cracking and final failure. The low fracture toughness of NFAs has been recognized as one of the most critical drawbacks of the alloys for engineering applications. As compared in the Figure 104, the high strength NFAs (LAF3C and 14YWT-SM11CR) display very low fracture toughness $\left(\mathrm{K}_{\mathrm{JQ}}\right)$ over the test temperature, while significantly higher fracture toughness are measured from those with relatively low strengths, 14YWT-SM1HR and ODS Eurofer 97 and are 
comparable to those of non-ODS FM steels. It is, however, noticed that the $\mathrm{K}_{\mathrm{JQ}}$ for ODS Eurofer 97 decreases rapidly above $400^{\circ} \mathrm{C}$ and falls in the low fracture toughness band of NFAs at $700^{\circ} \mathrm{C}$. This indicates that the general method or lowering the strength of a material to improve fracture toughness may not work for high temperature applications.

The $\mathrm{K}_{\mathrm{JQ}}$ values for $14 \mathrm{YWT}-\mathrm{SM} 10$ and LAF20 drop below the $100 \mathrm{MPa} \sqrt{\mathrm{m}}$ mark at above $200^{\circ} \mathrm{C}$, below which the fracture mode is in the upper shelf region. This sudden drop of fracture toughness at an elevated temperature is unique characteristics of NFAs whose strength is in the median range among NFAs. The $\mathrm{K}_{\mathrm{IQ}}$ data of the three FM steels show the typical temperature dependence of non-ODS steels that $\mathrm{K}_{\mathrm{JQ}}$ is high at low temperatures, since it is in the upper shelf region, and decreases with temperature gradually as strength decreases.

\subsubsection{Development of Dual Phase NFAs [INERI with Korea]}

T. S. Byun, byunts@ornl.gov, D. T. Hoelzer, hoelzerd@ornl.gov, ORNL

The main objective of this INERI project is to develop a nanoparticle strengthened dual phase composite for application to high performance reactor core structures. In the first year of the INERI collaboration, two base NFAs, Fe-9Cr-2W-0.4Ti-0.2V-0.12C-0.3 $\mathrm{Y}_{2} \mathrm{O}_{3}$ and Fe-9Cr-2W-0.4Ti-0.2V$0.05 \mathrm{C}-0.3 \mathrm{Y}_{2} \mathrm{O}_{3}$, were produced and named as 9YWTV-PM1 and 9YWTV-PM2, respectively. The extruded bars were cut into about 4 inch long coupons, as seen in Figure 105.

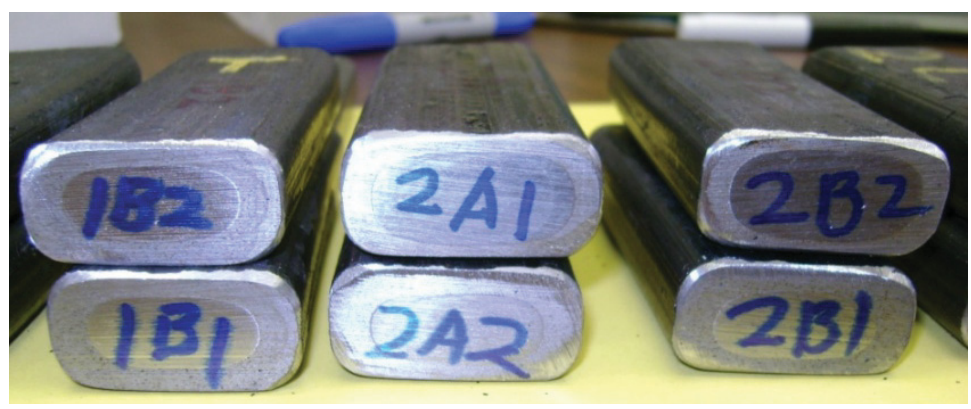

Figure 105. The coupons cut from the extruded bars. Note that in the coupons the inner meat part is the NFA and the outer rim part is from the carbon steel can.

SEM analysis was performed on polished specimens of 9YWTV-PM1 and 9YWTV-PM2 to investigate the grain structure. The BSE images shown in Figure 106 revealed the microstructures of both NFAs containing uniform distributions of ultra-small grains $<300 \mathrm{~nm}$. Detailed microstructural examination was performed for the as-extruded NFAs using XRD and field emission transmission electron microscope (FE-TEM). The nanoparticles within the grain are evenly scattered, whose mean diameter is about $2 \mathrm{~nm}$. Particles bigger than $10 \mathrm{~nm}$ exist rarely in the matrix and at the GB. The nanoparticles of about $2 \mathrm{~nm}$ in diameter are fully coherent with the matrix. 

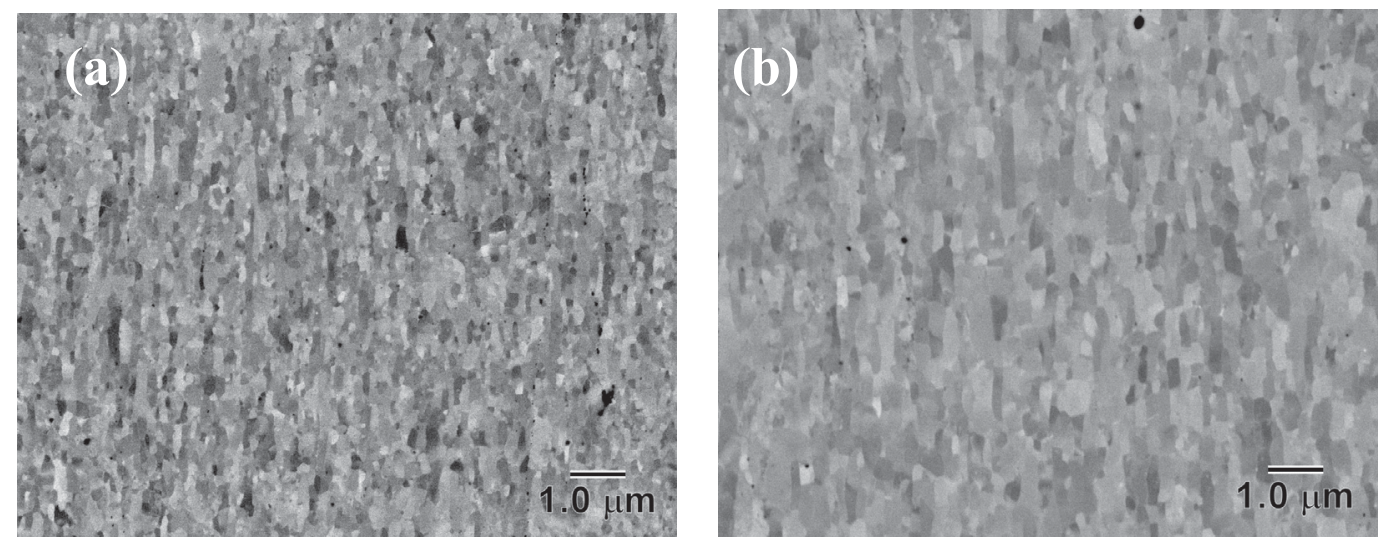

Figure 106. SEM BSE micrographs showing the ultra-fine grain structure of the extruded 9Cr NFAs: (a) 9YWTV-PM1 $(0.12 \% \mathrm{C})$ and (b) 9 YWTV-PM2 $(0.05 \% \mathrm{C})$.

Tensile tests have been carried out in the temperature range of $22-800^{\circ} \mathrm{C}$ in vacuum. The 9YWTVPM1 has slightly higher yield stress (YS) than the 14 YWT-SM10 up to $700^{\circ} \mathrm{C}$, while the 9YWTV-PM2 lower YS. Above $600^{\circ} \mathrm{C}$, however, the three alloys display similar strengths; it is notable that the new base NFAs retain YS higher than $500 \mathrm{MPa}$ at $700^{\circ} \mathrm{C}$ regardless of the difference in low temperature strength.

\subsubsection{Innovative Tube Processing for ODS Steels}

G. Grant, glenn.grant@pnnl.gov, PNNL

Nanostructured ferritics are anticipated to be strong contenders for use as fuel cladding in fast reactor high burn-up environments. A potential barrier to their use is the difficulties encountered in fabricating the material into a tubular product form required for cladding. These alloys are typically prepared through traditional powder metallurgical methods including mixing and ball milling or gas atomizing metal powders, then densifying through combinations of canning, hipping or extruding, then heat treated to produce the appropriate dispersoid microstructure and machining to final product. This multistep process is time consuming and the batch nature of the process results in a very high unit cost for the product.

This task is developing a technique called FCE to bypass most of the steps in traditional powder processing by using a method that densifies, shear processes, and extrudes product forms directly from powdered metals as show in Figure 107. An additional benefit to the process lies in the fact that it is a thermomechanical process that utilizes severe plastic deformation and as such, has the potential to impart desirable homogenization of powder components, dissolution then re-precipitation of desirable oxide dispersoids and at the same time impart grain size and textural features that may be desired in a cladding material. 


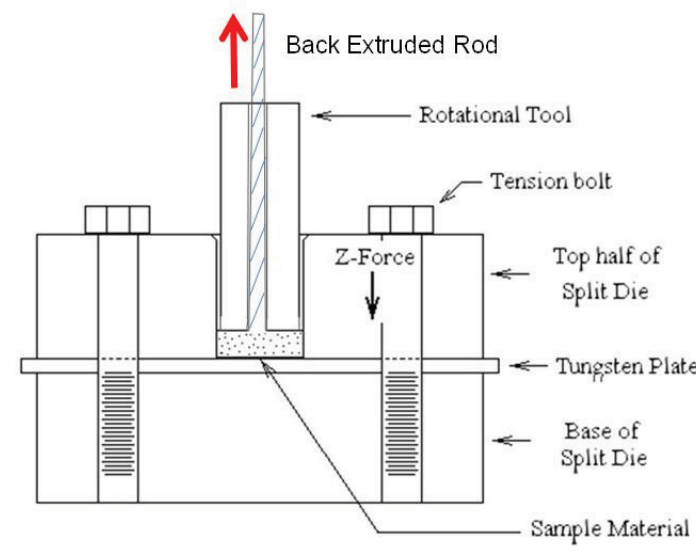

(a)

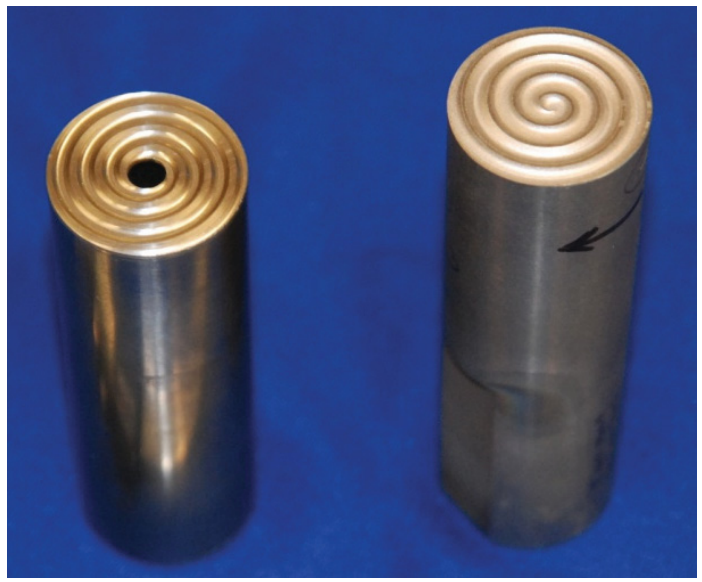

(b)

Figure 107. (a) Friction Consolidation and Extrusion concept and (b) Examples of plungers used to extrude rod (left) or compacted pucks (right).

Work during FY 2011 focused on demonstrating that ODS powders could be densified into pucks and that the desired dispersoids could by created by the process. Two alloys are being investigated in this work: MA956 and 14YWT. The MA956 powder used in these studies was prepared by traditional (high speed milling) methods, while the 14YWT was prepared by gas atomization. The MA956 powder, when analyzed, revealed that the oxide particle constituent elements (primarily Al-Y-O phases) were fully dissolved by ball milling. After densification of MA956 into a puck by FCE, the resulting microstructure was found have fully recrystallized very fine grains, and when analyzed in TEM were found to contain sub-10 nm dispersoids as shown in Figure 108. Work during the remaining part of FY 2011 focused on preparing for FCE trials on 14YWT and on efforts prepare a plunger to produce a rod product form using the FCE process.
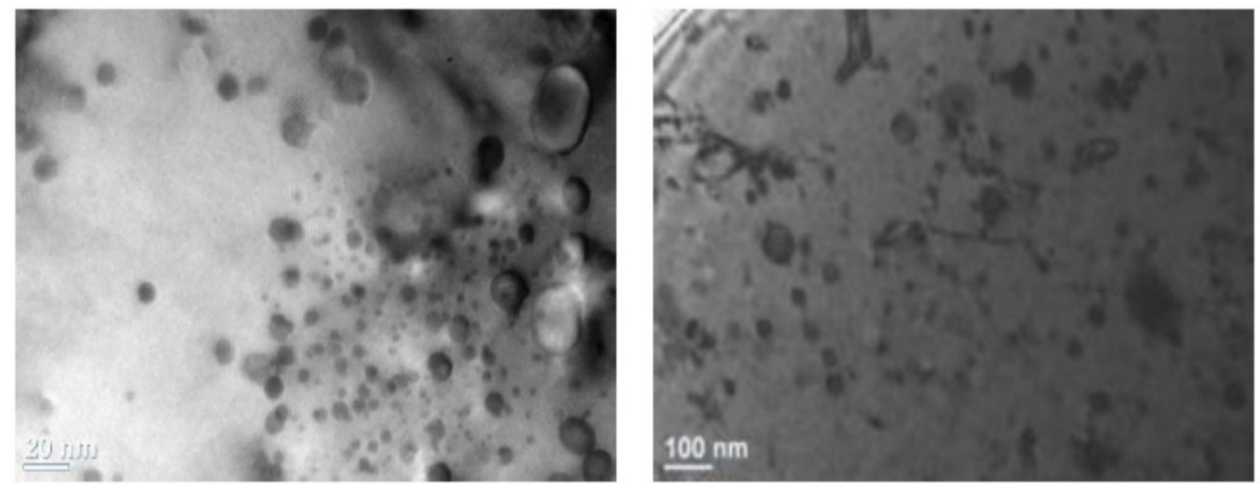

Figure 108. TEM micrographs and particle size distribution data on nanoscale dispersoids observed in FCE processed material (on left) verses material processed through traditional densification routes. The average dispersoid size in the FCE compact is $4.8 \mathrm{~nm}$, and $21.1 \mathrm{~nm}$ in the conventional product. 


\section{IRRADIATION TESTING TECHNOLOGES}

Technical Lead: S. Hayes, steven.hayes@inl.gov, INL

Progress was good during FY 2011 in the Irradiation Testing Technologies Technical Area, which includes both irradiation testing and PIE. Brief summaries from each of the work package managers are provided below; this introduction highlights the most important accomplishments for the year.

In the area of ATR Irradiations, the AFC-2D (high-burnup, MOX fuel with minor actinide additions experiment), AFC-2E (metallic fuel fabrication variables experiment), and gas-cooled fast reactor (GFR)F1 (legacy GFR matrix materials experiment) continued irradiation throughout the year in the East Flux Trap of ATR; they will all reach their target burnups/exposures mid-way through FY 2012 and be shipped to the Hot Fuels Examination Facility (HFEF) to begin PIE. Two new experiments, AFC-3A and AFC-3B (the advanced metallic fuel concept for reliable performance to ultra-high burnup experiment) were designed, analyzed, fabricated, and delivered to ATR and will begin irradiation in Cycle 150B (scheduled to start in October). Looking to the future, design work on AFC-4, an ATR irradiation vehicle better suited to support Separate Effects Testing (SET) with in-pile temperature measurement and control as well as the ability to accommodate advanced sensors for other in situ measurements, was initiated and progressed well; with the funding requested for FY 2012, the design of AFC-4 will be completed. In parallel with the AFC-4 irradiation vehicle design, $\mathrm{R} \& \mathrm{D}$ on a number of advanced sensor concepts were initiated.

In the area of HFIR Irradiations, preparations to initiate hydraulic rabbit testing made great strides. A series of reactivity tests were performed in HFIR, both during low-power reactor startups and at full power, to fully characterize the reactivity effects the fueled rabbit capsules would have on the reactor during rapid insertion and ejection. These tests enabled a conservative bound to be set on capsule content limits. It also identified some important changes needed to HFIR operating procedures in order to accommodate the fueled hydraulic rabbits, which also resulted in the preparation of a Safety Basis Supplement (SBS) to the HFIR Documented Safety Analysis (DSA). Approval of the SBS by the DOE Oak Ridge Operations Office (DOE-ORO) was received near the end of the year, clearing the way to initiate HFIR hydraulic rabbit testing early in FY 2012. In collaboration with INL, fabrication of the first set of rabbit capsules containing metallic fuel TEM specimens was completed. They are at ORNL and ready for use.

Finally, in the area of PIE, examinations at HFEF included a large array of non-destructive and destructive measurements performed on AFC-1 and AFC-2 series experiments (both metallic and oxide fuels) from recent ATR irradiation tests, as well as good progress on legacy oxide and metallic fuel pins from both FFTF and EBR-II. A Final PIE Report was prepared for the AFC-1D, 1G, 1H, and 2A experiments. Baseline PIE data collection was completed for $\mathrm{AFC}-2 \mathrm{~B}$ and $2 \mathrm{C}$, with analysis of the data underway and a PIE Report to be prepared in FY 2012. Very importantly, AFC-2C was the first oxide fuel experiment to be examined from the AFC test series; the characteristic microstructure observed in these rodlets (including central hole) gives great confidence that the Cd-shrouded ATR test configuration does indeed produce near-prototypic SFR temperature conditions in the test rodlets as designed.

\subsection{Advanced Test Reactor Irradiation Testing}

K. Barrett, kristine.barrett@inl.gov, INL

\section{AFC-2 Experiments}

During FY 2011, the AFC-2D, AFC-2E, and GFR-F1 experiments were inserted into the ATR EFT drop-in positions E1, E2, and E3 for irradiation during ATR operating cycles 148A, 148B, 149A, 149B, and 150B (see Figure 109). The GFR-F1 experiment was removed from position E3 at the end of cycle 
149B and transferred to the canal for storage to await shipment to HFEF for PIE. In support of these EFT irradiation tests, fabrication of six cadmium baskets was completed.

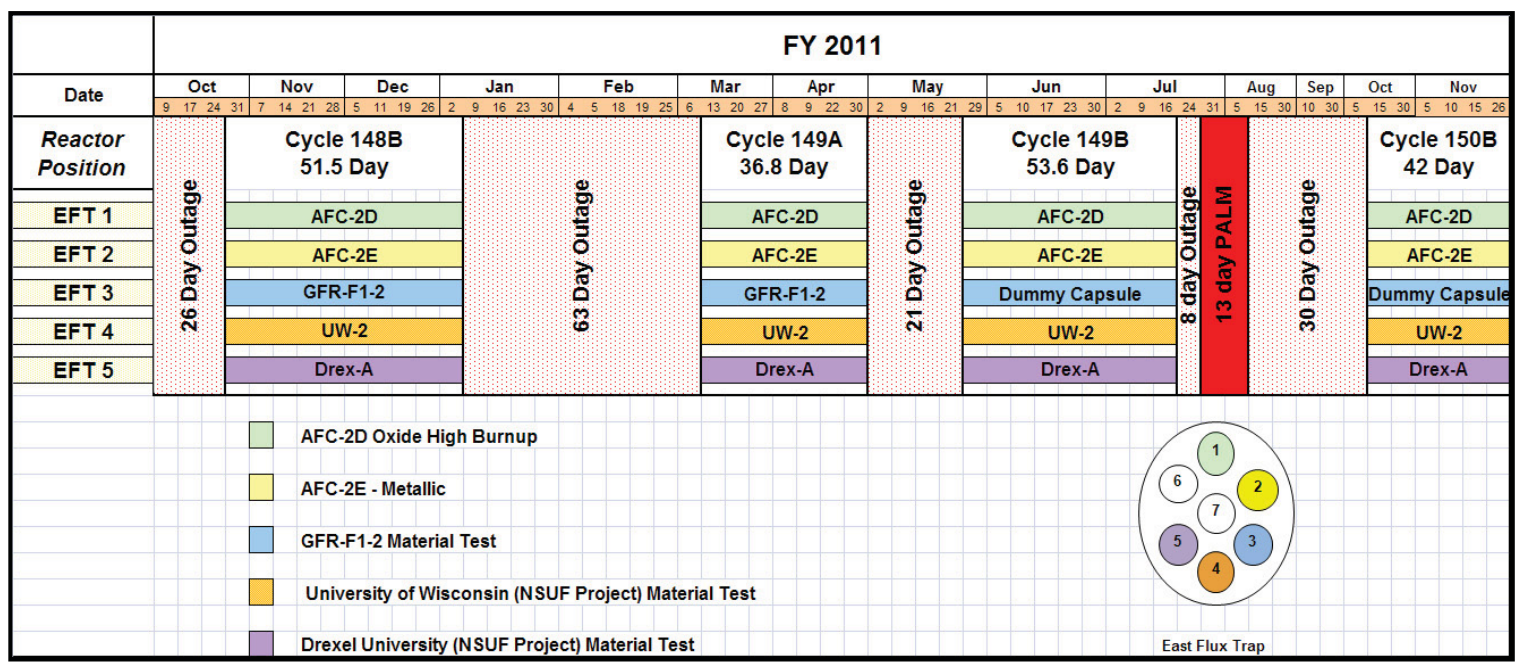

Figure 109. FY 2011 irradiation schedule for AFC-2 experiments.

Table 12 shows the as-run linear heat generation rate (LHGR) and burnup calculations for each rodlet in the AFC-2D and AFC-2E experiments for Cycle 149B, which was the last full cycle completed in FY 2011. Discharge of the AFC-2D and AFC-2E experiment assemblies is anticipated in the second quarter of FY 2012, or once desired burnup for each capsule is reached. Shipment of the AFC-2D, AFC-2E, and GFR-F1 capsules to HFEF for PIE is anticipated to occur in FY 2012.

Table 12. Cycle 149B As-Run LHGR, Fission Heat Rates, and Burnup Calculations for AFC Experiments in the EFT.

\begin{tabular}{|c|c|c|c|c|c|c|c|c|c|c|}
\hline \multicolumn{2}{|c|}{$\begin{array}{c}\text { Test Position } \\
\text { and ID }\end{array}$} & $\begin{array}{l}\text { Linear } \\
\text { Heat Rate } \\
\text { (W/cm) }\end{array}$ & $\begin{array}{c}\text { Fission } \\
\text { Heat Rate } \\
\text { (W/g) }\end{array}$ & $\begin{array}{l}\text { Fission } \\
\text { Density } \\
\text { (f/cc) }\end{array}$ & \begin{tabular}{|c|}
${ }^{239} \mathrm{Pu}$ \\
Depletion \\
$($ atom \%)
\end{tabular} & $\begin{array}{c}\text { Heavy } \\
\text { Metal } \\
\text { Depletion } \\
(\text { atom\%) }\end{array}$ & $\begin{array}{l}{ }^{241} \text { Am } \\
\text { Depletion } \\
(\text { atom \%) }\end{array}$ & \begin{tabular}{|c|}
${ }^{235} \mathrm{U}$ \\
Depletion \\
(atom\%)
\end{tabular} & \begin{tabular}{|c|}
${ }^{237} \mathrm{~Np}$ \\
Depletion \\
$($ atom\%)
\end{tabular} & $\begin{array}{l}{ }^{239} \mathrm{Pu}+ \\
{ }^{235} \mathrm{U} \\
\text { Depletion } \\
\text { (atom\%) }\end{array}$ \\
\hline \multirow{6}{*}{$\begin{array}{c}\text { E-1 } \\
\text { AFC-2D }\end{array}$} & Rodlet 1 & 198.17 & 119.76 & $2.09 \mathrm{E}+21$ & $22.07 \%$ & $12.55 \%$ & $53.35 \%$ & $23.76 \%$ & $26.94 \%$ & $23.17 \%$ \\
\hline & Rodlet 2 & 258.08 & 157.54 & $2.81 \mathrm{E}+21$ & $29.50 \%$ & $15.88 \%$ & $6.40 \%$ & $30.83 \%$ & -- & $30.37 \%$ \\
\hline & Rodlet 3 & 275.94 & 173.36 & $3.03 \mathrm{E}+21$ & $32.28 \%$ & $18.02 \%$ & $68.56 \%$ & $33.47 \%$ & $32.25 \%$ & $33.06 \%$ \\
\hline & Rodlet 4* & -- & -- & -- & -- & -- & -- & -- & -- & -- \\
\hline & Rodlet 5 & 260.91 & 161.78 & $2.83 \mathrm{E}+21$ & $29.87 \%$ & $16.92 \%$ & $66.15 \%$ & $31.64 \%$ & $32.39 \%$ & $31.02 \%$ \\
\hline & Rodlet 6 & 221.94 & 135.62 & $1.71 \mathrm{E}+21$ & $24.72 \%$ & $13.90 \%$ & $57.77 \%$ & $26.20 \%$ & $28.36 \%$ & $25.68 \%$ \\
\hline \multirow{6}{*}{$\begin{array}{c}\mathrm{E}-2 \\
\mathrm{AFC}-2 \mathrm{E}\end{array}$} & Rodlet 1 & 289.11 & 136.63 & $2.84 \mathrm{E}+21$ & $15.22 \%$ & $10.58 \%$ & -- & $14.50 \%$ & -- & $14.69 \%$ \\
\hline & Rodlet 2 & 307.78 & 147.82 & $3.07 \mathrm{E}+21$ & $18.69 \%$ & $11.02 \%$ & -- & $20.07 \%$ & -- & $19.63 \%$ \\
\hline & Rodlet 3 & 309.55 & 152.64 & $3.09 \mathrm{E}+21$ & $20.08 \%$ & $11.61 \%$ & $46.90 \%$ & $22.15 \%$ & $29.15 \%$ & $21.41 \%$ \\
\hline & Rodlet 4 & 334.00 & 156.88 & $3.25 \mathrm{E}+21$ & $20.91 \%$ & $11.32 \%$ & -- & $22.88 \%$ & -- & $22.12 \%$ \\
\hline & Rodlet 5 & 310.75 & 149.25 & $3.00 \mathrm{E}+21$ & $18.83 \%$ & $11.50 \%$ & $43.80 \%$ & $20.42 \%$ & $24.37 \%$ & $19.89 \%$ \\
\hline & Rodlet 6 & 257.63 & 120.76 & $2.55 \mathrm{E}+21$ & $15.99 \%$ & $9.15 \%$ & -- & $16.62 \%$ & -- & $16.42 \%$ \\
\hline
\end{tabular}

*Dummy Rodlet 


\title{
AFC-3 Experiments
}

During FY 2011, the AFC-3 experiment components were re-designed for use in the ATR OutboardA positions. Preliminary analysis of experiment components, based on AFC-2 design, failed to pass ATR safety analysis in FY 2011, primarily due to the smaller hole diameter of the Outboard-A positions compared to the EFT positions used for the AFC-2 experiments. Therefore, a significant effort was undertaken in FY 2012 to re-design experiment components to meet ATR safety requirements.

FY 2011 Accomplishments for the AFC-3 experiments included:

1. Completion of experiment design for use in ATR Outboard-A positions,

2. Completion of ATRC measurements in support of the final design,

3. Completion of the Experiment Safety Assurance Package (ESAP), experiment fabrication, and shipment to ATR complex.

At the end of FY 2011, AFC-3A and AFC-3B experiments were at ATR and approved for irradiation in Cycle 150B, expected to begin in early October.

\subsection{ATR Instrumented Lead for SET Design}

\author{
G. Hoggard, gary.hoggard@inl.gov, INL
}

The AFC-4 irradiation test series is the fourth series of Advanced Fuel Cycle (AFC) experiments undertaken by the FCRD program in the ATR. The AFC-4 test series seeks to evaluate fuels and materials irradiation behavior for advanced nuclear systems. These irradiation experiments will include a wide range of fuels, materials, and compositions in the form of small samples designed to evaluate separate effects. Fuel forms currently under investigation include metallic and ceramic systems. Fuel compositions may include binary and ternary metallic alloys and conventional oxide and MOX fuels, minor actinides to study transmutation, lanthanide additions to simulate reprocessing effects, and minor alloy additions to assess influence on a variety of performance parameters.

The objective of this series of experiments is to perform SET on nuclear fuel specimens in-pile to broadly advance the theoretical understanding of nuclear fuel behavior under irradiation and to inform/validate the complementary advanced modeling and simulation effort. This objective will be accomplished by adapting existing instrumented-lead experiment test train and temperature control system designs that have been successfully used in ATR. The use of these designs and some existing hardware and systems will significantly reduce the cost and accelerate the schedule for inserting the initial experiment in this series.

Design and analysis of the AFC-4 irradiation test train vehicle progressed well during FY 2011. Accomplishments include:

1. Developed and issued, "Project Execution Plan: Fuel Cycle Research and Development AFC4 Experiment" (INL-PLN-3676),

2. Developed and issued, "Technical and Functional Requirements: Fuel Cycle Research and Development AFC-4 Experiment In-Vessel Hardware" (TFR-741),

3. Documentation for tailoring the existing designs for AFC-4 progressed and neared completion,

4. Bounding thermal-hydraulic and structural analyses of the capsule design are underway,

5. Conceptual designs for the specimen holders (interface between the capsule and the specimens) were completed, and

6. An informal 30\% Design Review was conducted at year end. 


\title{
8.3 In-Pile Instrumentation Development
}

\author{
J. Rempe, joy.rempe@inl.gov, D. Knudson, J. Daw, and R. Schley, INL, \\ L. Bond, J. Coble, M. Good, and R. Meyer, PNNL
}

As part of the FCRD program objective to emphasize science-based, goal-oriented research, a strategic research program was launched to obtain the high fidelity, real-time, data required for characterizing the performance of new fuels during irradiation testing. The overarching goal of this initiative is to develop new test vehicles with new sensors of unprecedented accuracy and resolution that can obtain the required data. Prior laboratory testing and, as needed, irradiation testing of sensors in these capsules will be completed as part of this initiative to give sufficient confidence that the irradiation tests will yield the required data.

To ensure that this effort meets programmatic needs and incorporates input from a wide-range of stakeholders, efforts initially focused on developing a strategic plan that summarized FCRD irradiation test conditions, desired parameters for detection, and an overview of currently available in-pile instrumentation. Then, a workshop was held in which U.S. and foreign experts from fuels, irradiation, and instrumentation fields not typically associated with nuclear fuels research ranked candidate sensor technologies. The final version of the strategic plan, a Level 2 milestone, incorporates the consensus reached during the workshop with respect to recommendations for the path forward for accomplishing the goals of this research program. Specifically, the plan outlines a program that includes tasks to investigate three types of sensor technologies deemed to offer the most promise with respect to key attributes, such small size, accuracy, fine resolution, and longevity under harsh irradiation test conditions. After the strategic plan was issued, research was initiated on the sensor technologies recommended for investigation. Although each technology can supply sensors for measuring a wide range of parameters, FY 2011 efforts identified a single parameter for initial laboratory testing. In each area, subsequent steps were identified that are required to deploy this initially investigated sensor and other types of sensors in that technology area. The initial sensors identified for study in each area with key results obtained during FY 2011 are highlighted below.

\section{Ultrasonics Based Sensors}

Ultrasonics research is initially focusing on temperature detection at multiple locations with a single probe. Although the accuracy and resolution will depend on the ultrasonic thermometer (UT) selected, prior evaluations indicate that temperature measurements can be obtained with the required accuracy (2$3^{\circ} \mathrm{C}$ ) at multiple locations with a single small diameter probe (e.g., $1.0 \mathrm{~mm}$ or less). Literature review results were used to identify candidate component designs and materials, calibration/stabilization methods, fabrication techniques, and signal processing techniques. Existing INL ultrasonics equipment and commercial UT sensors (Figure 110) were used to identify and procure equipment required for subsequent testing of specialized UT probes, suitable for higher temperature, irradiation testing. In addition, a preliminary test matrix was developed for evaluating options with laboratory testing and, as needed, irradiation evaluations. By completing research required to meet the FCRD requirements for UTs, many of the challenges for other ultrasonics sensors will be resolved, expediting deployment of subsequent sensor types required for this program. 


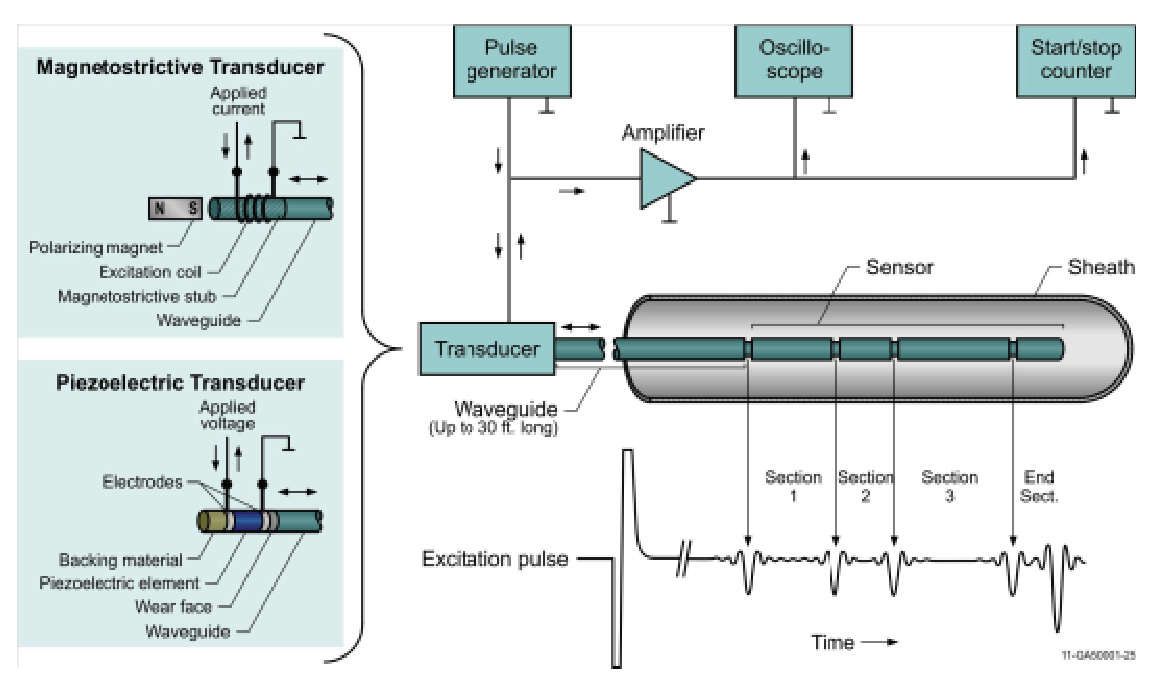

(a)

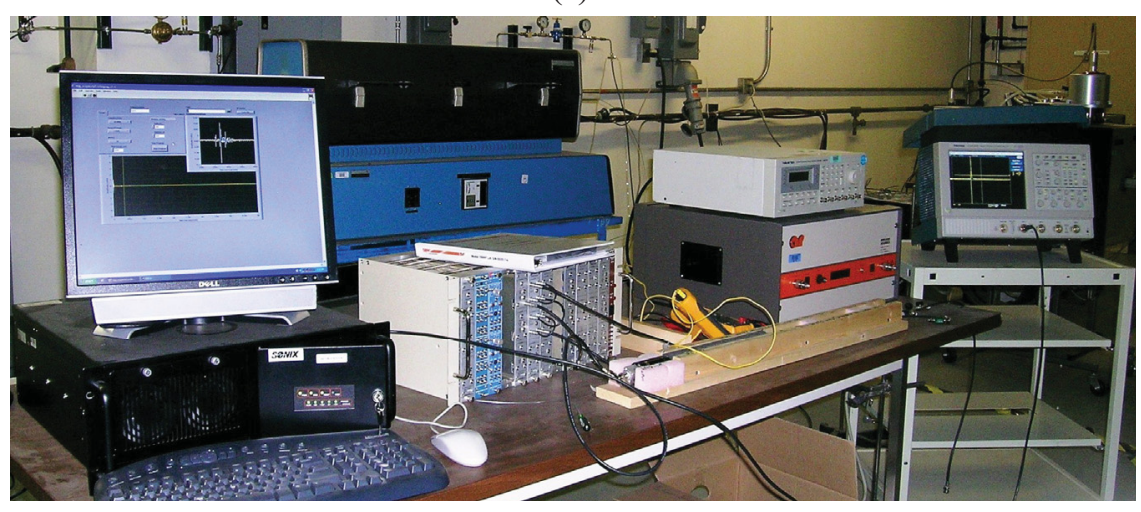

(b)

Figure 110. Ultrasonic thermometry efforts initiated in FY 2011 include (a) identifying candidate components for optimizing UT components and (b) evaluation of commercial UTs obtained from prior INL activities.

\section{Fiber Optics-Based Sensors}

Initial efforts in the fiber optics area are focusing on sensors for measuring changes in length. Laboratory evaluations of measurement techniques based on an Extrinsic Fabry-Perot Interferometer (EFPI) indicate elongations as small as $0.3 \mu \mathrm{m}$ can be detected for measurements ranging from 1.5 to 125 $\mu \mathrm{m}$. Based on results from this technique demonstration, a conceptual probe design (with a diameter of 1 $\mathrm{mm}$ ) was developed. Laboratory evaluations (Figure 111) verify the functionality of the small diameter concept sensor for detecting elongation, but additional work is needed to optimize the probe for deployment in an instrumented lead test and to demonstrate that fibers will not degrade during irradiation testing. 

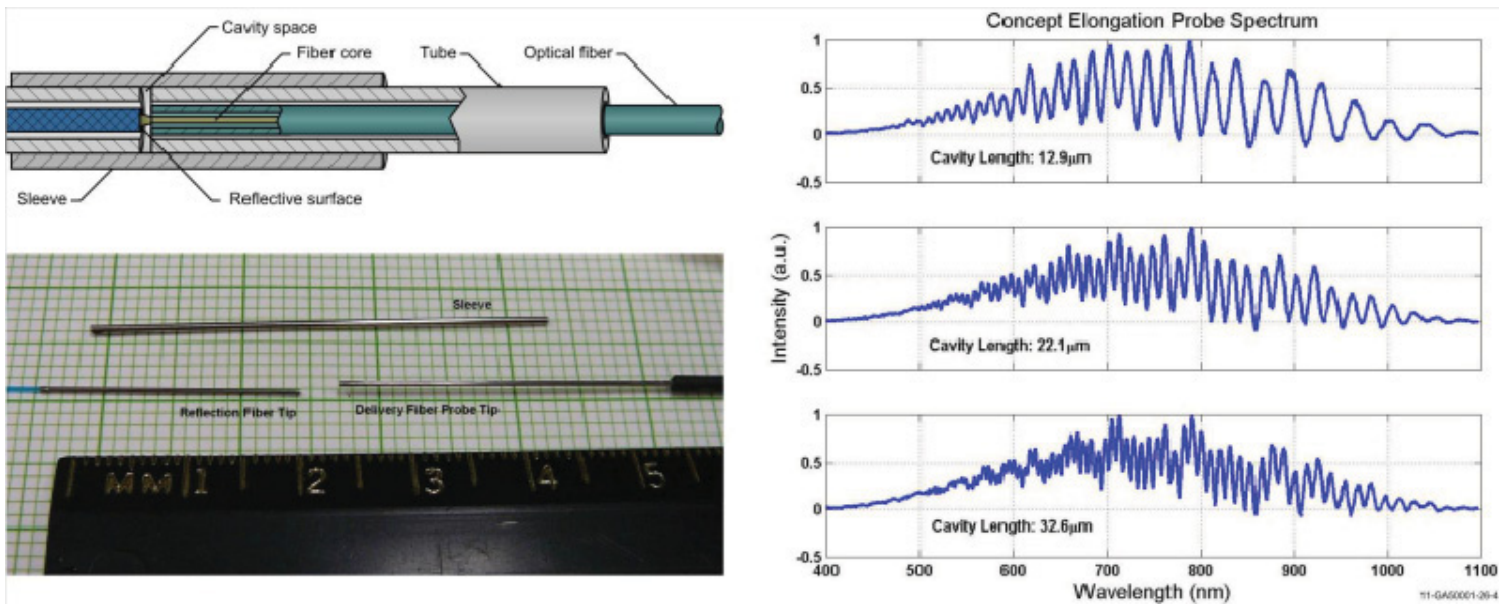

Figure 111. Initial fiber optics investigation (a) conceptual probe design, (b) conceptual probe components, and (c) spectra recorded using conceptual probe.

\section{Enhanced Existing U.S. MTR Sensors}

FCRD funding for enhancing existing in-pile instrumentation technology has been initially devoted toward deploying Linear Variable Differential Transformer (LVDT)-based sensors for detecting geometry changes. Building upon results from prior INL and Institute for Energy Technology at the Halden Reactor Project (IFE/HRP) collaborative efforts to deploy a creep test rig in a new PWR loop at the ATR, autoclave evaluations for a prototype creep test rig were completed (Figure 112). Comparisons with alternate methods (e.g., load frame and post-test measurements) indicate that this test rig can be used to measure elongations as small as $10 \mu \mathrm{m}$. Results from test rig evaluations were used to finalize recommendations for an enhanced design for insertion that will be inserted into the ATR in FY 2012. Data from the deployed creep test rig will be monitored in subsequent years as efforts are initiated to develop an enhanced creep test rig design in which the load to the specimen can be controlled and to develop an LVDT-based sensor for detecting change in fuel diameter during irradiation testing.
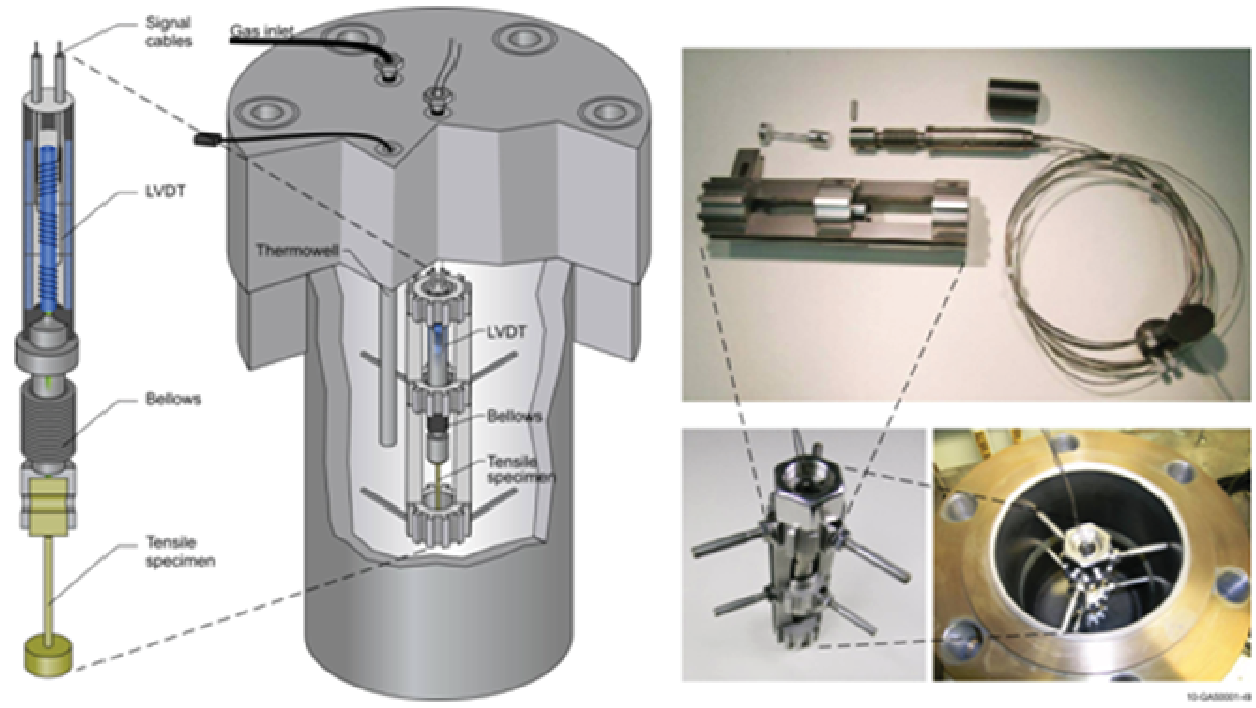

Figure 112. Creep test rig positioned in HTTL autoclave for testing. 


\section{Publications}

1. Rempe, J., D. Knudson, J. Daw, T. Unruh, B. Chase, and K. Davis, "Enhanced In-pile Instrumentation at the Advanced Test Reactor," invited paper, ANIMMA 2011 Special Edition, IEEE Transactions on Nuclear Science, submitted June 16, 2011.

2. Knudson, D. L., and J. L. Rempe, "LVDT-Based Elongation Measurements in Advanced Test Reactor High Temperature Irradiation Testing, Measurement Science and Technology, submitted September 2011.

3. Rempe, J., D. Knudson, J. Daw, T. Unruh, B. Chase, K. Condie, J. Palmer, and K. Davis, "Enhanced In-pile Instrumentation at the Advanced Test Reactor," Second International Conference on Advancements in Nuclear Instrumentation, Measurement Methods and their Applications (ANIMMA2011), Ghent, Belgium, June 2011.

4. Knudson, D., and J. Rempe, "Recommendations for use of LVDTs in ATR High Temperature Irradiation Testing,"7th International Topical Meeting on Nuclear Plant Instrumentation, Control, and Human Machine Interface Technologies (NPIC\&HMIT 2010), Las Vegas, NV, November 7-11, 2010.

5. Daw, J., J. Rempe, and S. Curtis Wilkins, "Ultrasonic Thermometry for In-Pile Temperature Detection," 7th International Topical Meeting on Nuclear Plant Instrumentation, Control, and Human Machine Interface Technologies (NPIC\&HMIT 2010), Las Vegas, NV, November 7-11, 2010.

\subsection{HFIR Irradiation Testing}

G. Bell, bellgl@ornl.gov, ORNL

The objective of this work is to perform irradiation testing of fuel materials using the HFIR Hydraulic Rabbit Test Facility. The ultimate goal of the effort is obtain a fundamental understanding of the evolution of the microstructure of nuclear fuel materials as a function of elemental composition, temperature and neutron fluence to better understand the in-service behavior and performance of the fuel. Toward this end, a series of low neutron fluence irradiation experiments have been planned to provide specimens for detailed PIE to generate data to support fuel modeling and simulation code development. The use of rabbit capsules inserted into and ejected from the HFIR core during full power (85 MW) operation provides for control of the neutron fluence, an important parameter in the evolution of the inservice properties of nuclear fuel materials. Appropriate design of the rabbit capsule provides not only for control of the temperature of the fuel specimen during irradiation, but control of the neutron spectrum as well.

To obtain the desired irradiation conditions, rabbits utilizing a strong thermal neutron absorber must be employed; however, rapid insertion and ejection of shielded (or "black") rabbits causes a reactivity response in the reactor that has the potential to engage the HFIR safety response system which could result in an unplanned shutdown, an event that must be studiously avoided. Therefore, a series of reactivity insertion experiments were performed to measure the margin to reactor trip at low power and full power to demonstrate that the risk of unplanned reactor shutdown is small and to define the acceptable operating space (i.e., rabbit reactivity worth, vertical position in the rabbit train, and number of rabbits having test articles). These experiments required a change to the HFIR Documented Safety Analysis (DSA), and DOE approval to conduct the testing was obtained (Oak Ridge Operations Office and Headquarters) in February 2011. The tests involved ejection of rabbits containing an internal gadolinium shield of the same diameter (0.262") to be used in the fuel sample irradiations with varying shield lengths (from 0.9" to 1.5") and measurement of the resultant reactor transient response (rate of rise of reactor power and reactor power level) on a high speed data recorder. The nominal calculated positive 
reactivity additions associated with ejection of the test rabbits were between 2.61 cents $(0.9$ " shield length) and 6.29 cents (1.5" shield length). The thermal and physics safety calculations were completed, all HFIR approvals were obtained, and the experiments were performed.

The results showed that reactor trip is prevented provided:

1. The length of the gadolinium shield is $<0.90$ " and the diameter is $<0.262$ ".

2. The irradiations are restricted to a single gadolinium shielded rabbit located in hydraulic tube position 7 or above.

3. Gadolinium shielded rabbit irradiations are restricted to the first 15 days of the operating cycle.

Since these controls are credited to prevent reactor trip by safety system action and have not been previously reviewed by DOE, DOE-ORO determined that DOE approval to perform the gadolinium shielded fuel sample rabbit irradiations was required. Another modification to the HFIR DSA was prepared and submitted to DOE-ORO and DOE Headquarters (DOE-HQ); approval was obtained in September 2011.

Following the black rabbit tests, the design of a rabbit capsule containing up to six fuel samples in the form of TEM disks ( $\sim 3 \mathrm{~mm}$ in diameter and $\sim 200$ um thick) was completed (Figure 113). For the first series of irradiations some of the specimen locations will be used to house temperature and flux monitors to provide as-run data and feedback on the design.

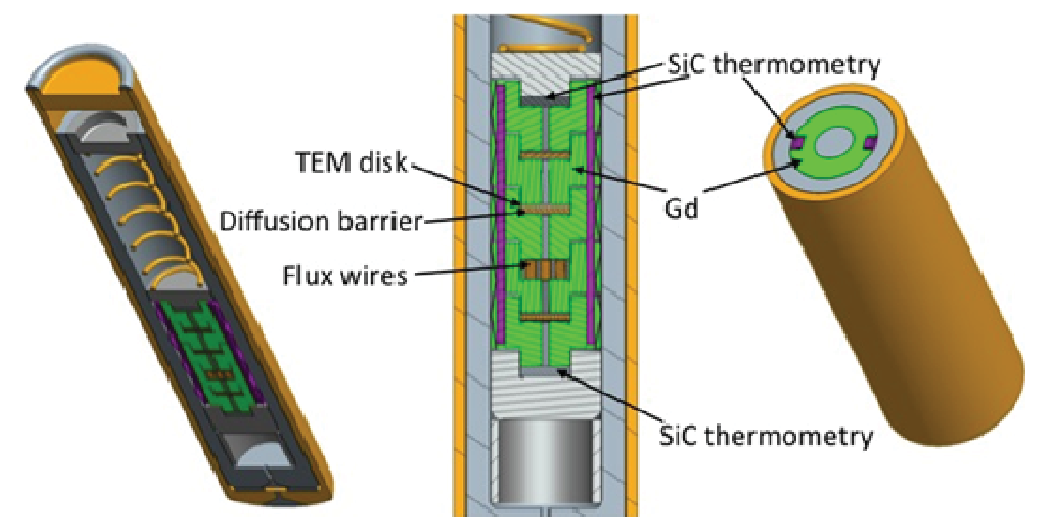

Figure 113. TEM rabbit design features the use of Gd spacers (0.9" stack length) that also serve as the thermal neutron shield. Space is provided for a maximum of 6 TEM disks samples, however SiC temperature monitors and flux monitors will displace some of the TEM disks as needed. The number of $\mathrm{Gd}$ spacers can be reduced to adjust the reactivity of the rabbit.

INL completed test article preparation and characterization of test articles and shipped twenty TEM disks (made from DU U8.2wt $\% \mathrm{Zr}, 0.21 \mathrm{wt} \%{ }^{235} \mathrm{U}$ ) to ORNL in May. The TEM disks were laser etched with identification markings to assist in the PIE effort, annealed, and loaded into rabbit capsules. Three TEM rabbit capsules (Figure 114) were loaded, welded up and leaked checked in June and are ready for irradiation in the HFIR flux trap, vertical position 7. The first rabbit irradiation is currently scheduled for late November 2011. 


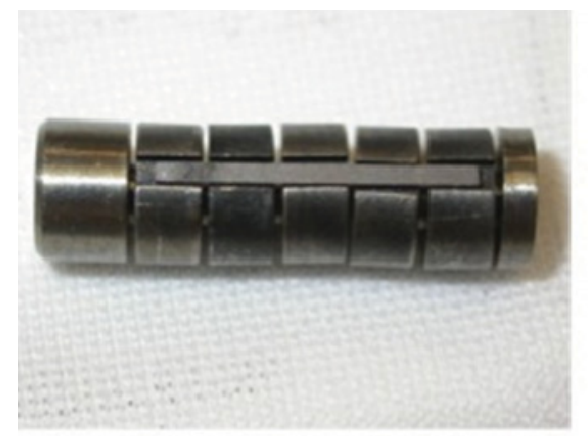

a) Inner Gd Stack - 1

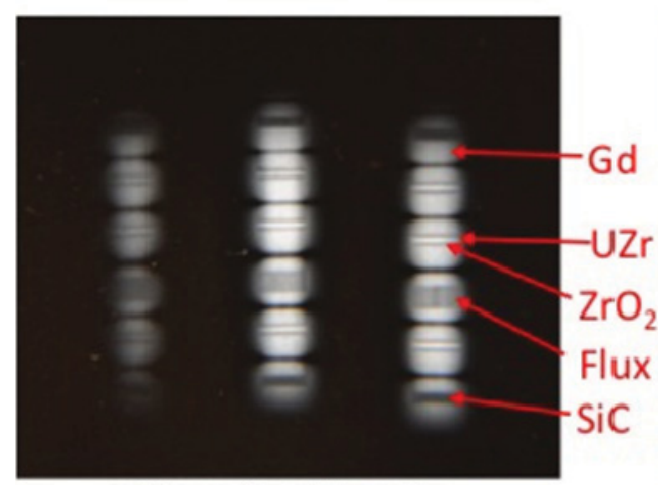

c) X-ray of loaded capsules

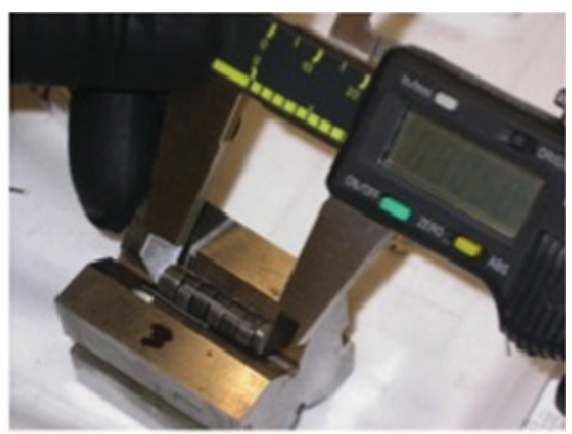

b) Inner Gd Stack - 2

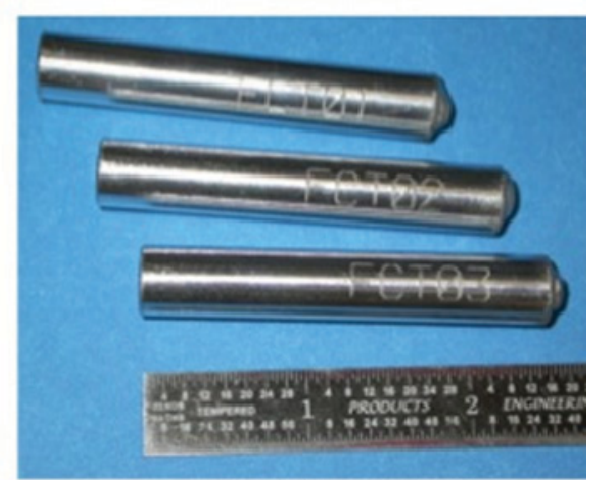

d) Loaded capsules

Figure 114. Images of the as-built TEM rabbit capsules: (a) photograph of the inner Gd stack with a SiC temperature monitor running the length of the stack, (b) photograph of the inner Gd stack being measured for length, (c) post-welding x-ray image of the three rabbit capsules, and d) photograph of the capsules prior to final cleaning step in preparation for irradiation.

In addition, physics calculations were performed to determine TEM disk displacements per atom to support experiment planning and to determine elemental/isotopic composition, radioactivity levels, and decay heat for the components of the TEM rabbit as a function of irradiation time and cooling time to address transportation and shipping information needs at HFIR and the INL.

\section{Publication}

1. G. L. Bell, J. McDuffee, R. W. Hobbs, L. J. Ott, R. J. Ellis, M. A. Okuniewski, and S. L. Hayes, "Preparations for Low Fluence Fuels Testing in the High Flux Isotope Reactor Hydraulic Rabbit Facility", OECD Nuclear Energy Agency Eleventh Information Exchange Meeting on Actinide and Fission Product Partitioning and Transmutation (San Francisco, CA, November 2010).

\subsection{Rabbit Test Definition}

\section{Okuniewski, maria.okuniewski@inl.gov, INL}

The objectives of this work were to: (1) define the irradiation test articles and parameters for the low fluence irradiations to be carried out in the HFIR Hydraulic Rabbit Test Facility and (2) coordinate these irradiation efforts between INL and ORNL. The ultimate goal of this research is to conduct low fluence irradiations to understand the early stages of microstructural evolution in fuels. This work will lead to a better scientific understanding of fuel performance. These experimental results will also enable the validation of computer models that are concurrently being developed.

A test plan was generated and submitted in May 2011 titled, "Test Plan for Metallic Fuel Irradiation in HFIR Hydraulic Rabbit". This plan detailed a variety of metallic alloys to be tested in the HFIR 
Hydraulic Rabbit Test Facility. These experiments are focused on conducting low fluence experiments to examine: (1) early stages of microstructural evolution in metallic fuels, (2) radiation enhanced diffusion and fuel cladding chemical interaction, (3) constituent redistribution, (4) thermophysical property evolution, and (5) historical EBR-II fuel irradiated in various facilities (e.g., fast and thermal spectra).

The initial specimens that will be inserted in HFIR were identified (DU U8.2wt $\% \mathrm{Zr}, 0.21 \mathrm{wt} \% \mathrm{U}^{235}$ ) and shipped to ORNL in May 2011. These specimens consisted of a typical transmission electron microscope specimen geometry (diameter of $3 \mathrm{~mm}$ and thickness of $200 \mu \mathrm{m}$ ). This initial specimen geometry will focus on the stages of microstructural evolution in metallic fuels. Moreover, these initial experiments that will be inserted into the HFIR hydraulic rabbit are meant to establish the proof of principle for utilizing this capability for short term irradiations. To establish the feasibility of this system, the initial tests were selected to be simplistic in design, while ensuring that the irradiation conditions (e.g., temperature and flux) are well instrumented and correspond to the calculations performed. The specimens were loaded into the capsules in June 2011. These specimens are anticipated for insertion at the reactor in November 2011. Additional irradiations of similar geometries and varying compositions are anticipated in FY 2012.

\subsection{Post Irradiation Examinations}

H. Chichester, heather.chichester@inl.gov, M. Teague, D. Porter, S. Hayes, J. Carmack, K Wright, INL

\section{AFG Fuel Experiments}

Baseline PIEs were completed on experiments AFC-1D, 1G, 1H, 2A, 2B, and 2C. Destructive exams of AFC-1D, 1G, 1H, and 2A were completed in FY 2011, including fission gas analysis, metallography, microhardness testing, and analytical chemistry. Nondestructive and destructive exams of AFC-2B and 2C were completed in FY 2011. Nondestructive PIE included visual inspection, dimensional measurements, neutron radiography, and gamma scans. Numerous breached rodlets were discovered and significant fuel-cladding chemical interaction was observed in AFC-2A and 2B metallography. Additional PIE is needed to investigate potential failure causes and beyond baseline PIE is planned to investigate constituent redistribution and elemental profiles. Optical microscopy of AFC-2C (Figure 115), the first MOX fuel irradiated in the AFC program, shows central void formation, a key indication that the fuel reached typical fast reactor temperatures during irradiation in ATR.

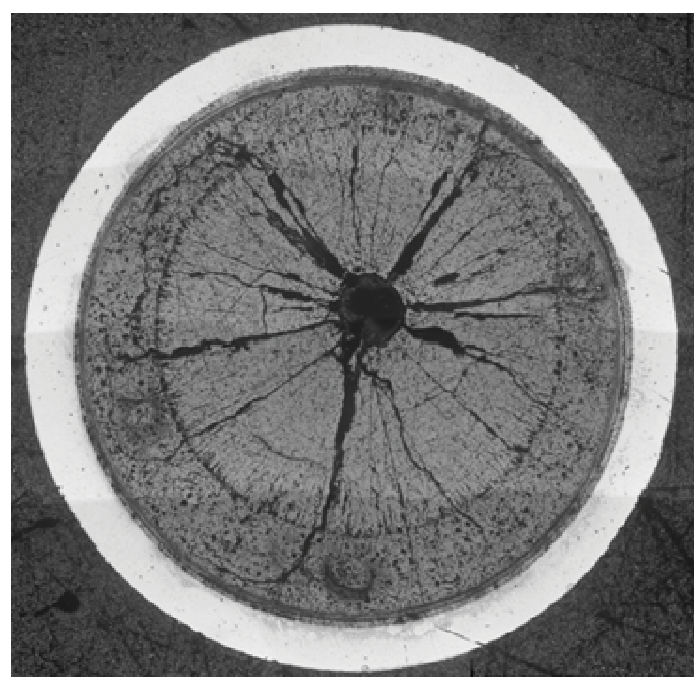

Figure 115. AFC-2C Rodlet 3 Fuel Cross-Section $\left(U_{0.75}, P u_{0.20}, A m_{0.05}, N p_{0.03}\right) O_{1.98}, 1.35 E 21 \mathrm{f} / \mathrm{cm}^{3}, 8.36$ at.\% HM burnup 


\section{FFT Legacy Fuel Examinations}

Baseline PIE of legacy FFTF annular oxide fuel was completed in FY 2011. The samples selected from fuel assemblies ACO-3 and FO-2 represent burnups between 3-24\% FIMA. The ACO-3 fuel is some of the highest burnup oxide fuel in existence. Ceramographic sections were cut and prepared from the highest burn-up fuel (Figure 116). Optical microscopy, micro-hardness, and fracture toughness measurements were obtained for the 6 sections cut from ACO-3 and FO-2 fuel pins. Characteristic high burnup or "rim" structure was observed in two of the three sections cut from the ACO-3 fuel pin. Further examination of the sections using advanced analytical techniques is underway.

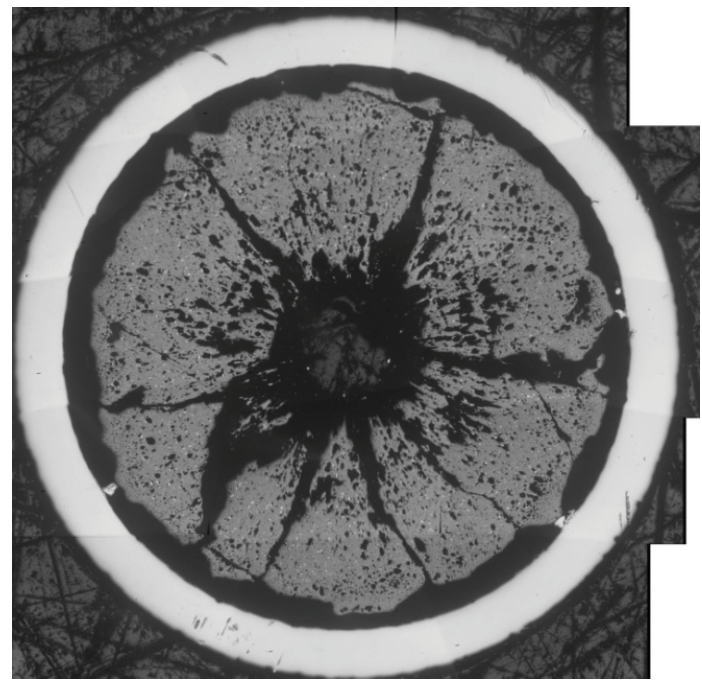

Figure 116. ACO-3 Fuel Cross Section 21\% FIMA Burnup

\section{EBR-II Legacy Fuel Examinations.}

Fuel pins from archived EBR-II experiments X501, an Am-bearing U-20Pu-10Zr fuel experiment, and X496, an experiment to test fuel of long-life design, were retrieved to continue PIEs. The X496 test contained fuel with very low smeared density (59\%) and an enlarged gas plenum designed to support testing of a fuel that could be run to very high burnup and perhaps fuel a once-through reactor design. While this is not a current FCRD concept some of the advanced fuel designs contain similar design features, and the behavior of fuel with a very low smeared density (gas release and fuel column stability, and fuel/cladding mechanical interaction) is important for benchmarking of fuel performance codes designed to predict these behaviors. Eight pins from X496 and one from X501 were retrieved for examination, but have not yet been examined. Neutron radiography (axial fuel swelling), gamma scanning (fission product redistribution), and plenum puncturing for gas pressure (fission gas release) may be done in FY 2012 if funding permits.

In addition, an old U-5Fs driver fuel pin from EBR-II was sectioned to examine the FCCI. Creep of the cladding, enhanced by cladding wall thinning from FCCI, is often the life-limiting phenomenon for metallic fuels. FCCI is known to be caused by interdiffusion of cladding components with RE fission products and some fuel alloy components. Examination of early documents describing FCCI involving U5Fs fuel revealed little mention of the participation of REs. If true, there may be clues to designing fuel with enhanced resistance to FCCI. The fuel section has just been examined using scanning electron microscopy with energy-dispersive x-ray spectroscopy. While the results have not yet been examined in detail, there is preliminary evidence that reveals why FCCI involving REs is minimized. If the results are confirmed, upcoming FCRD tests in ATR may prove the theory by showing no FCCI caused by REs. 


\section{EPMA Development}

During FY 2011, the EPMA was installed and performance tested on radioactive and non-radioactive samples. All software and hardware was found to be in satisfactory condition. In addition, a laboratory was set up for final specimen preparation, to include the use of a sample coater with a thickness monitor. Several candidates for actinide standards were prepared and tested for homogeneity. The Pu candidate will be an adequate standard for $\mathrm{Pu}$, while the $\mathrm{Pu}$-Am candidate will serve as a temporary standard for Am, until a single-element Am sample can be fabricated. A sample of a quantitative map made by the EPMA of a MOX pellet is shown below in Figure 117. Next steps will include analytical methods development for various radioactive sample types.
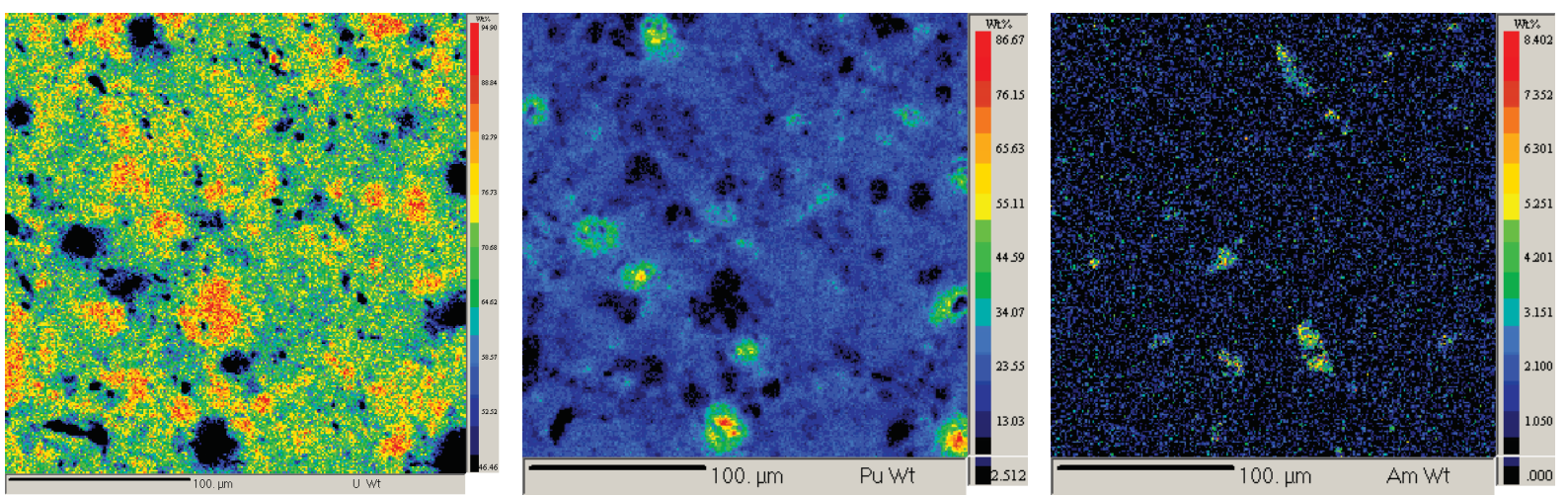

Figure 117. Quantitative Maps for a MOX Fuel Pellet from the EPMA. 